\title{
X-ray astronomy of stellar coronae ${ }^{\star}$
}

\author{
Manuel Güdel \\ Paul Scherrer Institut, Würenlingen and Villigen, 5232 Villigen PSI, Switzerland
}

Received 27 February $2004 /$

Published online 25 August 2004 - (C) Springer-Verlag 2004

\begin{abstract}
X-ray emission from stars in the cool half of the Hertzsprung-Russell diagram is generally attributed to the presence of a magnetic corona that contains plasma at temperatures exceeding 1 million K. Coronae are ubiquitous among these stars, yet many fundamental mechanisms operating in their magnetic fields still elude an interpretation through a detailed physical description. Stellar X-ray astronomy is therefore contributing toward a deeper understanding of the generation of magnetic fields in magnetohydrodynamic dynamos, the release of energy in tenuous astrophysical plasmas through various plasma-physical processes, and the interactions of high-energy radiation with the stellar environment. Stellar X-ray emission also provides important diagnostics to study the structure and evolution of stellar magnetic fields from the first days of a protostellar life to the latest stages of stellar evolution among giants and supergiants. The discipline of stellar coronal X-ray astronomy has now reached a level of sophistication that makes tests of advanced theories in stellar physics possible. This development is based on the rapidly advancing instrumental possibilities that today allow us to obtain images with sub-arcsecond resolution and spectra with resolving powers exceeding 1000 . High-resolution X-ray spectroscopy has, in fact, opened new windows into astrophysical sources, and has played a fundamental role in coronal research.

The present article reviews the development and current status of various topics in the X-ray astronomy of stellar coronae, focusing on observational results and on theoretical aspects relevant to our understanding of coronal magnetic structure and evolution.
\end{abstract}

Key words: X-rays: stars - Stars: coronae - Stars: flare - Stars: late-type - Stars: magnetic fields

For my part I know nothing with any certainty, but the sight of the stars makes me dream.

Vincent van Gogh (1853-1890)

\footnotetext{
* This article is dedicated to the late Rolf Mewe, a prominent astrophysicist who contributed major work to the field of stellar X-ray astronomy and spectroscopy. He died on May 4, 2004.
} 


\section{Contents}

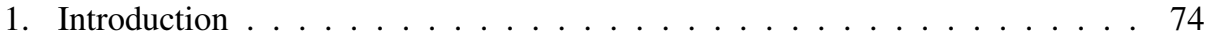

2. The study of stellar coronae . . . . . . . . . . . . . . . . . 77

3. The early days of stellar coronal X-ray astronomy . . . . . . . . . . . 78

4. A walk through the X-ray Hertzsprung-Russell diagram . . . . . . . . . . . . 81

4.1. Main-sequence stars . . . . . . . . . . . . . . . . . 81

4.2. The coolest $\mathrm{M}$ dwarfs . . . . . . . . . . . . . . . . 84

4.3. Brown dwarfs (and planets?) . . . . . . . . . . . . . . 85

4.4. A-type stars . . . . . . . . . . . . . . . 86

4.4.1. X-ray emission from normal A stars . . . . . . . . . . 86

4.4.2. Chemically peculiar A stars . . . . . . . . . . . . . . 87

4.4.3. Herbig Ae/Be stars . . . . . . . . . . . . . . . . 88

4.5. Giants and supergiants . . . . . . . . . . . . . . . . . . . 88

4.6. Close binaries . . . . . . . . . . . . . . . . . . . 89

4.7. Contact binary systems . . . . . . . . . . . . . . . . . 89

5. X-ray activity and rotation . . . . . . . . . . . . . . . . 90

5.1. Rotation-activity laws . . . . . . . . . . . . . . . . 90

5.2. Activity and rotation in stars with shallow convection zones . . . . . 92

5.3. Rotation and saturation; supersaturation . . . . . . . . . . . . . 92

5.4. Physical causes for saturation and supersaturation . . . . . . . . . . 93

5.5. Rotation and activity in pre-main sequence stars, giants and binaries . 94

6. Flux-flux relations . . . . . . . . . . . . . . . . . 95

6.1. Chromosphere-transition region-corona . . . . . . . . . . . 95

6.2. Radio - X-ray correlations . . . . . . . . . . . . . . . 96

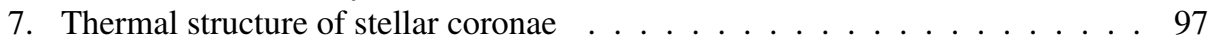

7.1. Thermal coronal components . . . . . . . . . . . . . . . . . 97

8. High-resolution X-ray spectroscopy . . . . . . . . . . . . . . . . . 98

9. The differential emission measure distribution . . . . . . . . . . . . . . . 100

9.1. Theory ...................... 100

9.2. Interpretation ..................... 100

9.2.1. The DEM of a static loop . . . . . . . . . . . . . . 100

9.2.2. The DEM of flaring structures . . . . . . . . . . . . 102

9.3. Reconstruction methods and limitations . . . . . . . . . . . . 103

9.4. Observational results . . . . . . . . . . . . . . . . . 107

9.5. Coronal temperature-activity relations . . . . . . . . . . . . 108

10. Electron densities in stellar coronae . . . . . . . . . . . . . . . . . 110

10.1. Densities from Fe line ratios . . . . . . . . . . . . . . 110

10.2. Line ratios of He-like ions . . . . . . . . . . . . . . . . . . . 112

10.3. Spectroscopic density measurements for inhomogeneous coronae . . 115

11. The structure of stellar coronae . . . . . . . . . . . . . . . . . . 117

11.1. Loop models . . . . . . . . . . . . . . . . . . . . 117

11.2. Coronal structure from loop models . . . . . . . . . . . . . . . 119

11.2.1. Loop parameters . . . . . . . . . . . . . . . . . . . . 119

11.2.2. Loop-structure models . . . . . . . . . . . . . . . . 119

11.2.3. Conclusions and limitations ............ . . 121 
11.3. Coronal structure from densities and opacities . . . . . . . . . . . . . 122

11.4. Coronal constituents: Emission-measure interpretation . . . . . . . . 123

11.5. Coronal imaging: Overview . . . . . . . . . . . . . . . . . . . . 124

11.6. Active-region modeling . . . . . . . . . . . . . . . 125

11.7. Maximum-entropy image reconstruction . . . . . . . . . . . . 126

11.8. Lucy/Withbroe image reconstruction . . . . . . . . . . . . . . . . 127

11.9. Backprojection and Clean image reconstruction . . . . . . . . . . . 128

11.10. Coronal structure inferred from eclipses . . . . . . . . . . . . . 128

11.10.1. Extent of eclipsed features . . . . . . . . . . . . . . 128

11.10.2. Structure and location of coronal features . . . . . . . . . . 131

11.10.3. Thermal properties of coronal structures . . . . . . . . . 131

11.11. X-ray coronal structure in other eclipsing binaries . . . . . . . . . 132

11.12. Inferences from rotational modulation . . . . . . . . . . . . . 133

11.13. Rotationally modulated and eclipsed X-ray flares . . . . . . . . . . . 134

11.14. Inferences from Doppler measurements . . . . . . . . . . . . . . 136

11.15. Inferences from surface magnetic fields . . . . . . . . . . . . . 137

11.16. Extended or compact coronae? . . . . . . . . . . . . . . . . . 138

12. Stellar X-ray flares . . . . . . . . . . . . . . . . . . . . . 139

12.1. General properties and classifications . . . . . . . . . . . . . . 140

12.2. General flare scenario . . . . . . . . . . . . . . . . . 141

12.3. Cooling physics . . . . . . . . . . . . . . 141

12.4. Interpretation of the decay time . . . . . . . . . . . . . 142

12.5. Quasi-static cooling loops ... . . . . . . . . . . . . 144

12.6. Cooling loops with continued heating . . . . . . . . . . . . 145

12.7. Two-Ribbon flare models . . . . . . . . . . . . . . . . . . . . . . . . . . . . . 147

12.8. Hydrodynamic models . . . . . . . . . . . . . . . . . . . 149

12.9. Magnetohydrodynamic models . . . . . . . . . . . . . . 151

12.10. Summary of methods . . . . . . . . . . . . . . . . 152

12.11. Observations of stellar X-ray flares . . . . . . . . . . . . 153

12.12. Flare temperatures . . . . . . . . . . . . . . . 153

12.13. Flare densities . . . . . . . . . . . . . . . . . . . . 159

12.14. Correlation with UV and optical flares . . . . . . . . . . . . . 161

12.15. Correlation with radio flares . . . . . . . . . . . . . . 162

12.16. The "Neupert Effect" . . . . . . . . . . . . . . . . . . 162

12.17. Non-thermal hard X-rays? . . . . . . . . . . . . . . . . . 164

13. The statistics of flares . . . . . . . . . . . . . . . . . 165

13.1. Correlations between quiescent and flare emissions . . . . . . . . 165

13.2. Short-term coronal X-ray variability . . . . . . . . . . . . 166

13.3. Stochastic variability - what is "quiescent emission"? . . . . . . . . 166

13.4. The solar analogy . . . . . . . . . . . . . . . . . 168

13.5. The flare-energy distribution . . . . . . . . . . . . . . . 170

13.6. Observables of stochastic flaring . . . . . . . . . . . . . 172

14. X-ray absorption features and prominences . . . . . . . . . . . . . 173

15. Resonance scattering and the optical depth of stellar coronae . . . . . . . . . 174

16. The elemental composition of stellar coronae . . . . . . . . . . . . . 175

16.1. Solar coronal abundances: A brief summary . . . . . . . . . . . . 176 
16.2. Stellar coronal abundances: The pre-XMM-Newton/Chandra view . . 177

16.3. Stellar coronal abundances: New developments with XMM-Newton and Chandra . . . . . . . . . . . . . . . . . . . 178

16.4. Systematic uncertainties . . . . . . . . . . . . . . . 182

16.5. Coronal and photospheric abundances . . . . . . . . . . . . . . 183

16.6. Flare metal abundances . . . . . . . . . . . . . . . . . . . 186

16.7. Theoretical models for abundance anomalies . . . . . . . . . . . . 187

17. X-ray emission in the context of stellar evolution . . . . . . . . . . . . . 188

17.1. Main-sequence stars . . . . . . . . . . . . . . . . 188

17.2. Giants . . . . . . . . . . . . . . . . . . 190

17.3. Dividing lines . . . . . . . . . . . . . . . . . 192

17.4. Hybrid stars . . . . . . . . . . . . . . . . . 193

17.5. Evolution of X-ray emission in open stellar clusters . . . . . . . . . . 194

17.5.1. Overview . . . . . . . . . . . . . . . . 194

17.5.2. Rotation-age-activity relations . . . . . . . . . . 196

17.5.3. Binaries . . . . . . . . . . . . . . . . 199

17.5.4. Co-eval clusters . . . . . . . . . . . . . . . 200

17.5.5. Toward older clusters . . . . . . . . . . . . . 201

17.5.6. Toward younger clusters .............. 201

18. X-ray coronae and star formation . . . . . . . . . . . . . . . . . 201

18.1. T Tauri stars . . . . . . . . . . . . . . . . . 202

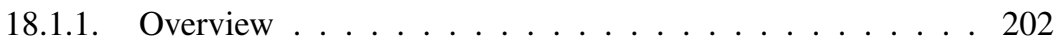

18.1.2. X-ray luminosity and age . . . . . . . . . . . 203

18.1.3. X-ray luminosity, saturation, and rotation . . . . . . . . . . 204

18.1.4. The widely dispersed "field WTTS" samples . . . . . . . . 205

18.1.5. Flares .................. 206

18.1.6. The circumstellar environment . . . . . . . . . . . . . . 207

18.1.7. Accretion-driven X-ray emission? . . . . . . . . . . . 207

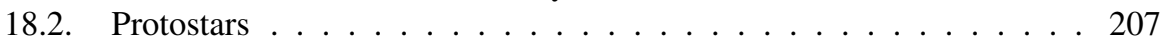

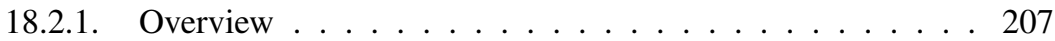

18.2.2. Flares and magnetic fields . . . . . . . . . . . . . . 208

18.2.3. The stellar environment . . . . . . . . . . . . . 209

18.2.4. "Class 0 " objects ... . . . . . . . . . . . 210

18.3. Young brown dwarfs ................... . 211

19. Young populations in the solar neighborhood . . . . . . . . . . . . . . 213

20. Long-term variability and stellar magnetic cycles . . . . . . . . . . . . . . 213

20.1. Clusters and field star samples . . . . . . . . . . . . . . . . . 214

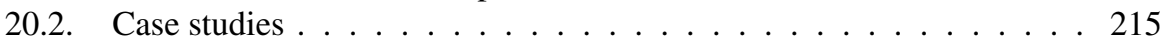

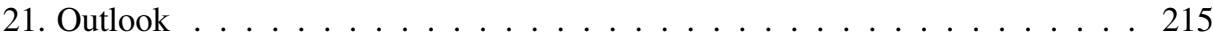

\section{Introduction}

More than half a century ago, the presence of a very hot, tenuous gas surrounding the Sun, the $X$-ray corona, was inferred indirectly from optical coronal lines of highly ionized species (Grotrian 1939; Edlén 1942) and more directly by detecting X-ray photons in 
the course of a rocket flight (Burnight 1949) ${ }^{1}$. Around the same time, radio observations revealed a radio corona as well (Hey 1946). Of course, the sheer beauty of the solar corona has been admired in scattered visible light ever since humans first wondered about solar eclipses, but only during the last very few decades have we started to seriously grasp solutions to some of the fundamental astrophysical problems within the field of solar and stellar astronomy.

Magnetic fields have come to the center of our attention in this endeavor. They seem to be ubiquitous among stars, but neither do we understand precisely why, nor have we fully understood the bewildering variety of plasma physical mechanisms that act in stellar environments. We have found magnetic fields on stars that ought to have none so long as we appeal to our limited understanding of magnetic field production and amplification. We witness various processes of energy transport and energy release intimately related to those very magnetic fields; the fields not only guide mass and energy flows, they are the sources of energy themselves. But our understanding of energy dissipation has remained patchy, in particular in magnetically active stars.

The coronal magnetic fields reach into the stellar environment, structuring it and governing heating and particle acceleration. Nevertheless, except in the case of the Sun, we have very little, and usually only indirect evidence of the topology of magnetic fields. In very young stars, magnetic fields may reach out to the circumstellar accretion disks. Again, their role is manifold: they transport angular momentum and thus control the spin rate of the star. They guide mass flows, thus take a leading role in the mass accretion process. They release energy and thus ionize the stellar molecular environment, possibly altering the physics and chemistry of accretion disks and thereby influencing the formation of planets. At later stages, they control stellar rotation through angular momentum transport via a stellar wind and thus engage in a feedback loop because the magnetic field production is rooted in precisely this rotation.

The magnetic field thus plays a fundamental role in the evolution of the radiative environment of a star, with far-reaching consequences for the chemical development of planetary atmospheres and, eventually, the formation of life. These and many further challenges have stimulated our field of research both in theory and observation, producing a rich treasure of ideas and models from stellar evolution to elementary plasma-physical processes that reach way beyond specific coronal physics problems.

Yet, it has proven surprisingly difficult to study these magnetic fields in the outer stellar atmospheres and the stellar environments. Thus, the essence of a stellar corona is not yet accessible. It is the mass loading of magnetic fields that has given us specific diagnostics for magnetic activity to an extent that we often consider the hot plasma and the accelerated high-energy particles themselves to be our primary subjects.

Although often narrowed down to some specific energy ranges, coronal emission is intrinsically a multi-wavelength phenomenon revealing itself from the meter-wave radio range to gamma rays. The most important wavelength regions from which we have learned diagnostically on stellar coronae include the radio (decimetric to centimetric) range and the X-ray domain. The former is sensitive to accelerated electrons in magnetic

\footnotetext{
1 T. Burnight wrote, "The sun is assumed to be the source of this radiation although radiation of wave-length shorter than 4 angstroms would not be expected from theoretical estimates of black body radiation from the solar corona." The unexpected has prevailed all through the history of coronal physics indeed!
} 
fields, and that has provided the only direct means of imaging stellar coronal structure, through very long baseline interferometry.

The most productive spectral range for stellar coronal physics has, however, been the soft X-ray domain where the mysteriously heated bulk plasma trapped in the coronal fields radiates. The X-ray diagnostic power has been instrumental for our understanding of physical processes in coronae, and the recent advent of high-resolution X-ray spectroscopy with the Chandra and XMM-Newton X-ray observatories is now accessing physical parameters of coronal plasma directly.

This review is predominantly concerned with the soft X-ray domain of stellar coronal physics, and partly with the closely related extreme ultraviolet range. Radio aspects of stellar coronae have been reviewed elsewhere (Güdel 2002) and will be occasionally addressed when they provide complementary information to our present subject.

Notwithstanding the importance of these two wavelength regimes, I emphasize that further diagnostics are available at other photon energies. Although optical and ultraviolet spectroscopy refers predominantly to cooler layers of stellar atmospheres, a few coronal emission lines detected in this wavelength range promise some complementary diagnostics in particular through the very high spectral resolving power available. And second, the hard X-ray and $\gamma$-ray range, recognized as the fundamental source of information for energy release physics in the solar corona, will be of similar importance for stars although, at the time of writing, it remains somewhat of a stellar terra incognita, waiting for more sensitive instruments to detect these few elusive photons.

A review of a field that has accumulated massive primary literature from three decades of continuous research based on numerous satellite observatories, necessarily needs to focus on selected aspects. The review in hand is no exception. While trying to address issues and problems across the field of stellar X-ray astronomy, I have chosen to put emphasis on physical processes and diagnostics that will help us understand mechanisms not only in stars but in other astrophysical environments as well. Understanding energyrelease physics, magnetic-field generation mechanisms, and evolutionary processes of magnetic structures from protostars to giants will eventually contribute to our understanding of the physics in other astrophysical objects. Examples are accretion-driven mechanisms in disks around active galactic nuclei, the physics of large-scale galactic magnetic fields, or the heating and cooling of galaxy cluster gas.

In the course of this review, a number of controversial issues and debates will deliberately be exposed - this is where more investment is needed in the future. Also, while I will address some topics relatively extensively, I will touch upon others in a somewhat more cursory way. My hope is that various previous reviews help close the gaps. They themselves are far too numerous to name individually. I would recommend, among others, the following reviews as entry points to this field: The extreme-ultraviolet domain has recently been summarized extensively by Bowyer et al. (2000). Haisch et al. (1991a) summarized the multi-wavelength view of stellar and solar flares. Two early comprehensive reviews of X-ray astrophysics of stellar coronae were written by Rosner et al. (1985) and Pallavicini (1989), and very recently Favata and Micela (2003) have presented a comprehensive observational overview of stellar X-ray coronae.

An interesting early summary of nonradiative processes in outer stellar atmospheres that comprises and defines many of our questions was given by Linsky (1985). Mewe (1991) reviewed X-ray spectroscopic methods for stellar coronae. High-energy aspects 
in the pivotal domain of star formation and early stellar evolution were comprehensively summarized by Feigelson and Montmerle (1999).

Topics that are - despite their importance for stellar astronomy - predominantly the subject of solar physics are not discussed here. In particular, plasma-physical mechanisms of coronal heating, the acceleration of (solar and stellar) winds, and the operation of internal dynamos will only be touched upon in so far as stellar observations are contributing specifically to our knowledge. I also note that insights relating to our subject provide diagnostics that reach out to entirely different fields, such as studies of the rotational history of stars, the ionization structure and large-scale evolution of star-forming molecular clouds, the structure and the composition of galactic stellar populations, and dynamo theory for various types of stellar systems. Beyond what I can address in this summary, I need to refer the reader to the more specialized literature.

\section{The study of stellar coronae}

The study of stellar coronae, of course, starts with the Sun. This provides a rather important advantage for stellar astronomers: that of having a nearby, bright standard example available at high spatial and spectral resolution. What, then, should we expect from the study of stellar coronae?

In the context of the solar-stellar connection, stellar X-ray astronomy has introduced a range of stellar rotation periods, gravities, masses, and ages into the debate on the magnetic dynamo. Coronal magnetic structures and heating mechanisms may vary together with variations of these parameters. Parameter studies could provide valuable insight for constraining relevant theory. Different topologies and sizes of magnetic field structures lead to different wind mass-loss rates, and this will regulate the stellar spin-down rates differently. As is now clear, on the other hand, rotation is one of the primary determinants of the magnetic dynamo. This point could not be demonstrated by observing the Sun: The Sun's magnetic activity is in fact strongly modulated (due to the 11-year magnetic spot cycle, Fig. 1), but this effect is not directly dependent on the rotation period. Conversely, the well-studied solar activity cycle motivates us to investigate similar magnetic modulations in stars in order to confine the underlying dynamo mechanism.

Stars allow us to study long-term evolutionary effects by observing selected samples with known and largely differing ages. While models of the solar interior and its evolution predict that the young Zero-Age Main-Sequence (ZAMS) Sun was fainter by $\approx 25 \%$ than at present, stellar X-ray astronomy has revealed that the solar high-energy emission was likely to be hundreds of times more intense at such young ages. The increased level of ionizing and UV radiation must then have had an important impact on the formation and chemistry of planetary atmospheres.

At younger stages still, the radiation at short wavelengths may have been pivotal for ionizing the circumstellar accretion disk. This must have resulted in at least five significant effects that determine the further process of planet formation and stellar evolution. First, stellar magnetic fields couple to the inner disk and thus guide mass accretion onto the star; second, and at the same time, torques mediated by the magnetic fields regulate the rotation period of the star; third, winding-up magnetic fields between star and disk may release further magnetic energy that may lead to jet (and indirectly, to molecular outflow) activity; fourth, permeation of the weakly ionized disk by magnetic 


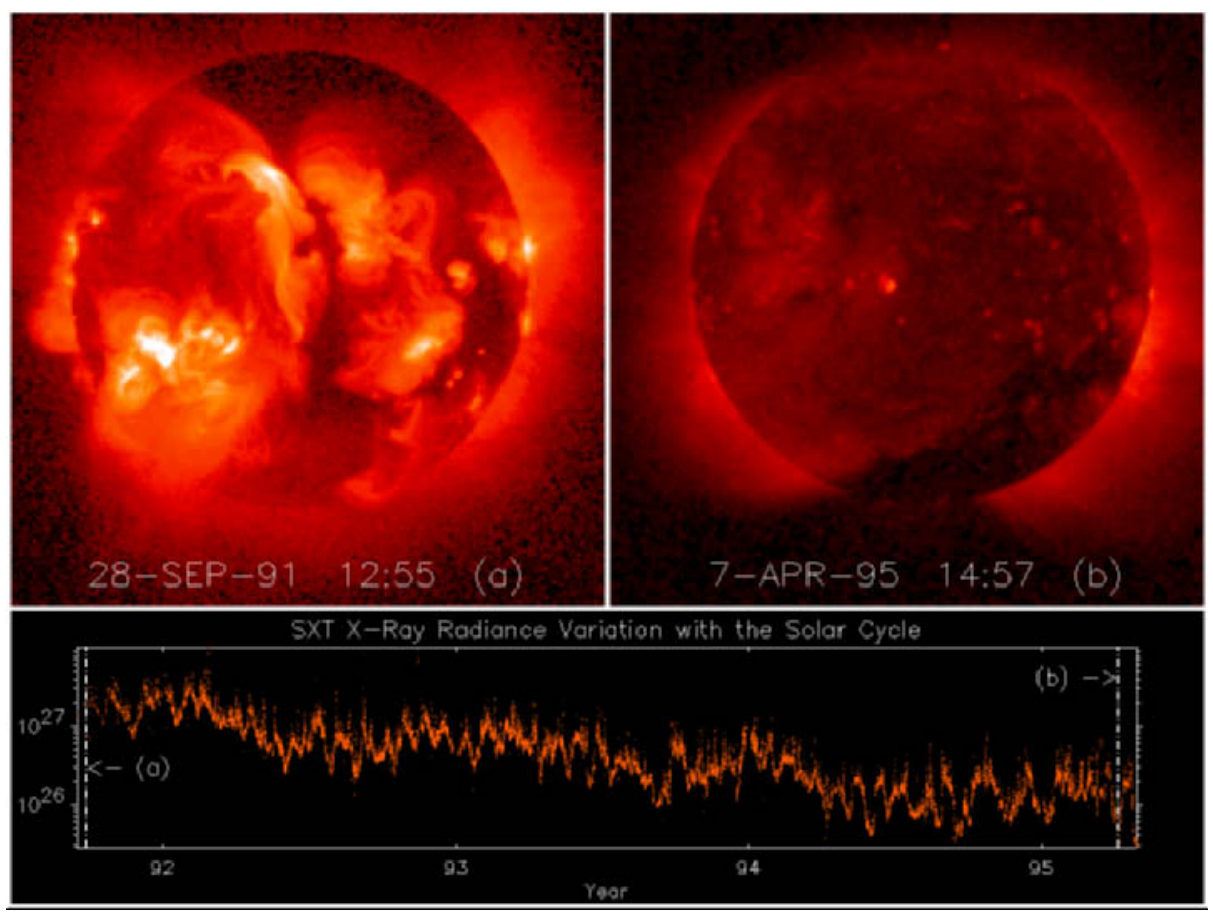

Fig. 1. Yohkoh images of the Sun during activity maximum (left, in 1991) and minimum (right, in 1995). The light curve in the lower panel illustrates the long-term "cyclic" variability of the solar soft X-ray output

fields induces instabilities (e.g., the Balbus-Hawley instability) that are relevant for the accretion process and planet formation; and fifth, the X-ray irradiation may directly influence the disk chemistry and thus the overall evolution of the dust disk (e.g., Glassgold et al. 2000).

Including stars into the big picture of coronal research has also widened our view of coronal plasma physics. While solar coronal plasma resides typically at $(1-5) \times 10^{6} \mathrm{~K}$ with temporary excursions to $\approx 20 \mathrm{MK}$ during large flares, much higher temperatures were found on some active stars, with steady plasma temperatures of several tens of MK and flare peaks beyond $100 \mathrm{MK}$. Energy release in stellar flares involves up to $10^{5}$ times more thermal energy than in solar flares, and pressures that are not encountered in the solar corona.

\section{The early days of stellar coronal X-ray astronomy}

While this review is entirely devoted to (non-solar) stellar studies, an important anchor point would be missing if the success of the Skylab mission in the early seventies were not mentioned. The high-quality images of the full-disk Sun in X-rays formed, together with data from previous rocket flights, our modern picture of the solar and therefore stellar coronae. The solar X-ray corona is now understood as a dynamic ensemble of magnetic 
loops that contain hot plasma in ever-changing constellations, yet always related to the underlying photospheric magnetic field. Interacting loops may episodically evolve into flares that release much of their energy as soft X-rays. Coronal holes, characterized by low X-ray emission and predominantly open magnetic field lines along which the solar wind escapes at high speed, fill volumes between bright coronal active regions. From a stellar astronomer's point of view, the early solar coronal studies culminated in the formulation of scaling laws for coronal structures. Best known is the seminal paper by Rosner et al. (1978) in which several of the basic ideas of coronal structure and static loops were developed or extended. These concepts paved the way to interpreting stellar coronae without requiring the imaging capabilities that have been so central to solar studies.

The field of stellar coronal X-ray astronomy was in fact opened around the same time, in 1975 when Catura et al. (1975) reported the detection of Capella as the first stellar coronal X-ray source on the occasion of a rocket flight. They estimated the X-ray luminosity at $10^{31} \mathrm{erg} \mathrm{s}^{-1}$ - four orders of magnitude above the Sun's - and the plasma electron temperature at about $8 \times 10^{6} \mathrm{~K}$, again several times higher than the Sun's. I note in passing that the latter measurement is in quite close agreement with modern values. This result was confirmed by Mewe et al. (1975) from observations with the ANS satellite; they were the first to interpret the soft X-rays as solar-like coronal emission at an enhanced level. Around the same time, Heise et al. (1975) monitored the first stellar coronal X-ray flares (on YZ CMi and UV Cet) with ANS; one of the flares was recorded simultaneously with an optical burst. The possible contributions of stellar X-ray flares to the diffuse galactic soft X-ray background and of associated particles to the cosmicray particle population were immediately recognized and discussed. Numerous flare observations followed, opening up new avenues of research on energy release physics familiar from the Sun. For example, White et al. (1978) related a soft X-ray flare on HR 1099 with a simultaneous radio burst.

Further detections followed suit. Algol was next in line, defining another new class of stellar X-ray sources (Schnopper et al. 1976; Harnden et al. 1977). The initial discussion related the X-rays to an accretion mass stream, however, while modern interpretation ascribes them to coronal structures on the K-type secondary. A series of detections with HEAO 1, namely of RS CVn and HR 1099 (Walter et al. 1978a), Capella (Cash et al. 1978), and UX Ari (Walter et al. 1978b) established the RS CVn binaries as a class of coronal X-ray sources that may also be significant contributors to the galactic soft X-ray background. The unusually high X-ray production of this class was confirmed in the survey by Walter et al. (1980a), also based on HEAO 1 .

Main-sequence (MS) stars were brought on stage with the first discovery of Proxima Centauri in the extreme ultraviolet range by Haisch et al. (1977), while Nugent and Garmire (1978) identified the "solar twin" $\alpha$ Cen as an inactive coronal source at a luminosity level similar to the Sun. Several further single MS stars at the high end of the activity scale were found, putting our own Sun into a new perspective as a rather modest X-ray star (Walter et al. 1978c; Cash et al. 1979a; Walter et al. 1980b). Discoveries soon extended the coronal range into the A spectral class (Mewe et al. 1975; Topka et al. 1979) although these early detections have not been confirmed as coronal sources, from the present-day point of view. (Mewe et al. 1975 suggested the white dwarf in the Sirius system to be the X-ray source.) 
An important new chapter was opened with the introduction of medium-resolution X-ray spectroscopy. Cash et al. (1978) used HEAO 1 to obtain the first coronal X-ray spectrum of Capella. They correctly interpreted excess emission between 0.65 and $1 \mathrm{keV}$ as being due to the Fe XVII/XVIII complex. The earliest version of a (non-solar) stellar coronal emission measure distribution can be found in their paper already! They noticed that the high temperature of Capella's corona requires magnetic confinement unless we are seeing a free-flowing coronal wind. Another element of modern coronal X-ray interpretation was introduced by Walter et al. (1978b) when they realized that subsolar abundances were required to fit their medium-resolution spectrum of UX Ari. The first explicit X-ray spectra of (non-solar) stellar flares were obtained by Kahn et al. (1979). The thermal nature of the emission was confirmed from the detection of the $6.7 \mathrm{keV} \mathrm{Fe}$ $\mathrm{K} \alpha$ line.

The Einstein satellite revolutionized the entire field of coronal X-ray astronomy, transforming it from a domain of mostly exotic and extreme stars to a research area that eventually addressed X-ray emission from all stars across the Hertzsprung-Russell diagram, with no lack of success in detecting them either as steady sources (Vaiana et al. 1981) or during large outbursts (Charles et al. 1979). The field of star formation entered the scene when not only star formation regions as a whole, but individual young stars such as T Tau stars were detected as strong and unexpectedly variable X-ray sources (Ku and Chanan 1979; Ku et al. 1982; Gahm 1980; Walter and Kuhi 1981), including the presence of strong flares (Feigelson and DeCampli 1981).

The solid-state spectrometer on board Einstein also provided spectroscopic access to many coronal X-ray sources and identified individual emission-line blends of various elements (Holt et al. 1979). These spectra permitted for the first time multi-temperature, variable-abundance spectral fits that suggested the co-existence of cool and very hot ( $\gtrsim 2 \times 10^{7} \mathrm{~K}$ ) plasma in RS CVn binaries (Swank et al. 1981; Agrawal et al. 1981).

Lastly, grating spectroscopy started to resolve individual spectral lines or blends in coronal X-ray spectra. Mewe et al. (1982) described spectroscopic observations of Capella that separated $\mathrm{Fe}$ and $\mathrm{O}$ lines in the 5-30 A region using the Einstein objective grating spectrometer with a resolving power up to at least 30 . Only few high-resolution stellar spectra were obtained with the Focal Plane Crystal Spectrometer (FPCS) on Einstein and the Transmission Grating Spectrometer (TGS) on EXOSAT. The instruments offered spectral resolving powers of 50-500 in the X-ray band (FPCS) and of $\$ 60$ in the EUV band (TGS), respectively. The former instrument was used in narrow wavelength bands only; examples include observations of $\sigma^{2} \mathrm{CrB}$ (Agrawal et al. 1985) and Capella (Vedder and Canizares 1983). While these instruments marked a breakthrough in Xray spectroscopy at the time, the $\mathrm{S} / \mathrm{N}$ achieved was not sufficient to derive detailed information on emission measure (EM) distributions. Nevertheless, rough models were derived that grossly resemble the results from modern high-sensitivity spectroscopy (Vedder and Canizares 1983). Later, the TGS was used to derive information on the EM distribution of bright X-ray sources (Lemen et al. 1989) and to study loop models (Schrijver et al. 1989b).

The ultimate breakthrough in stellar coronal physics came with the initial Einstein survey that led to three significant insights. First, X-ray sources abound among all types of stars, across the Hertzsprung-Russell diagram and across most stages of evolution (Vaiana et al. 1981). Stars became one of the most prominent classes of cosmic X-ray 
sources. Second, the X-ray luminosities and their distribution now uncovered along the main sequence could not be in agreement with the long-favored acoustic heating theories; the X-ray emission was now interpreted as the effect of magnetic coronal heating. And third, stars that are otherwise similar reveal large differences in their X-ray output if their rotation period is different (Pallavicini et al. 1981; Walter and Bowyer 1981). These systematics have been in the center of dynamo theory up to the present day, and it is fair to say that no dynamo theory will be deemed fully successful without addressing the latter two points in some detail.

The initial findings were rapidly consolidated (e.g., Johnson 1981; Ayres et al. 1981a) but cool-star X-ray astronomy has remained an active research area to the present day, with no lack of debate. The following chapters are devoted to our still exciting era of stellar X-ray astronomy.

\section{A walk through the X-ray Hertzsprung-Russell diagram}

A look at the Hertzsprung-Russell diagram (HRD) of detected X-ray stars in Fig. 2, compiled from selected catalogs of survey programs (Alcalá et al. 1997; Berghöfer et al. 1996; Hünsch et al. 1998a,b, 1999; Lawson et al. 1996), shows all basic features that we know from an optical HRD (we plot each star at the locus of the optically determined absolute magnitude $M_{\mathrm{V}}$ and the color index $B-V$ regardless of possible unresolved binarity). Although the samples used for the figure are in no way "complete" (in volume or brightness), the main sequence is clearly evident, and so is the giant branch. The top right part of the diagram, comprising cool giants, is almost devoid of detections, however. The so-called corona vs. wind dividing line (dashed in Fig. 2; after Linsky and Haisch 1979) separates coronal giants and supergiants to its left from stars with massive winds to its right. It is unknown whether the wind giants possess magnetically structured coronae at the base of their winds - the X-rays may simply be absorbed by the overlying wind material (Sect. 17.3). The few residual detections may at least partly be attributed to low-mass companions. The large remaining area from spectral class $\mathrm{M}$ up to at least mid-F comprises stars that are - in the widest sense - solar-like and that define the subject of this review. I now turn to a few selected domains within the HRD that have attracted special attention. The domain of star formation and pre-main sequence evolution will be discussed in a wider context toward the end of this review (Sect.18).

\subsection{Main-sequence stars}

The main sequence (MS henceforth) has arguably played the most fundamental role in the interpretation of stellar magnetic activity. It is here that we find a relatively clear correspondence between mass, radius, and color. On the other hand, evolutionary processes map poorly on the MS, providing us with a separate free parameter, namely age or, often equivalently, rotation rate. Current wisdom has it that the most massive coronal MS stars are late-A or early F stars, a conjecture that is supported both by observation and by theory. Theory predicts the absence of a magnetic dynamo in earlier A stars, given the 


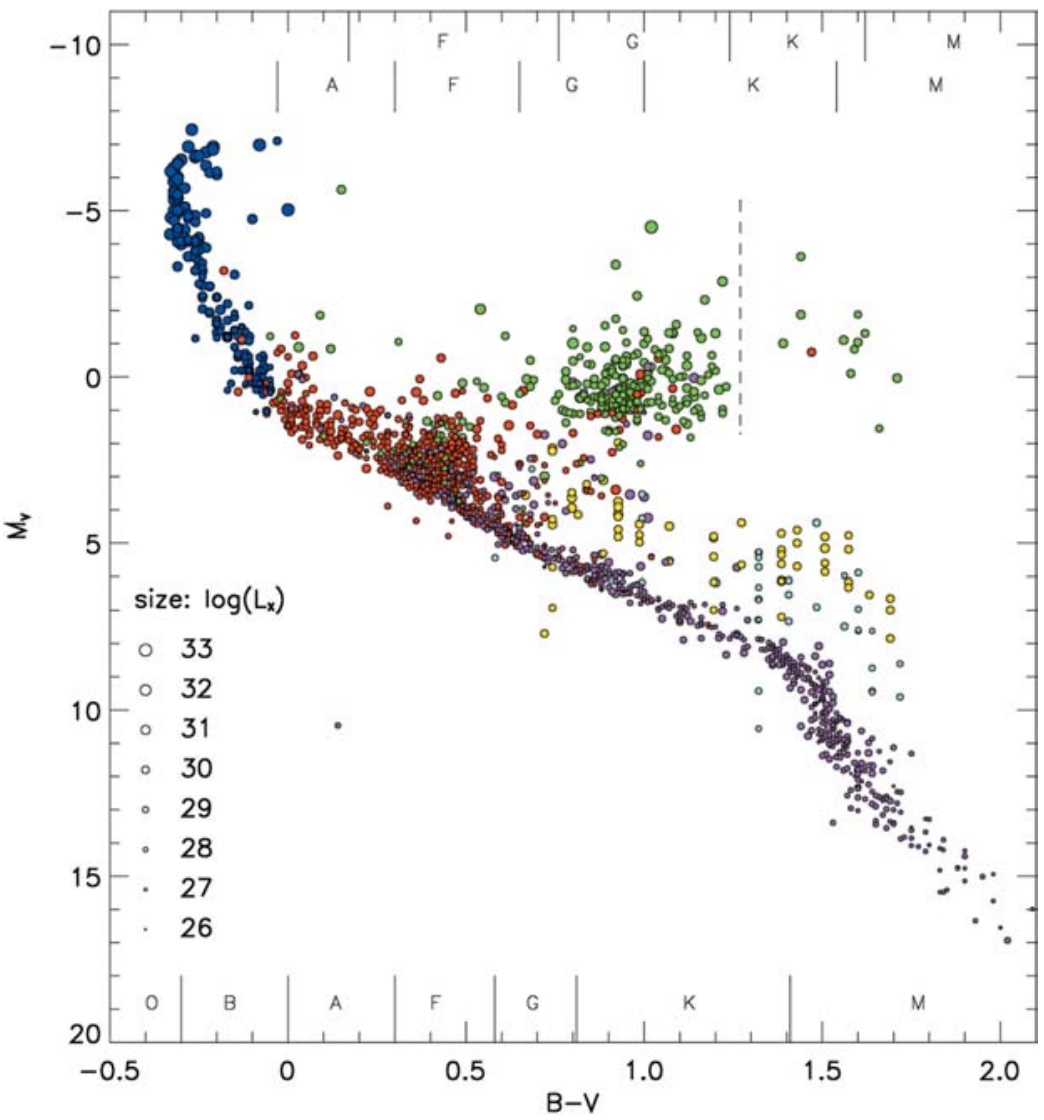

Fig. 2. Hertzsprung-Russell diagram based on about $2000 \mathrm{X}$-ray detected stars extracted from the catalogs by Berghöfer et al. (1996) (blue), Hünsch et al. (1998a,b) (green and red, respectively), and Hünsch et al. (1999) (pink). Where missing, distances from the Hipparcos catalog (Perryman et al. 1997) were used to calculate the relevant parameters. The low-mass pre-main sequence stars are taken from studies of the Chamaeleon I dark cloud (Alcalá et al. 1997; Lawson et al. 1996, yellow and cyan, respectively) and are representative of other star formation regions. The size of the circles characterizes $\log L_{\mathrm{X}}$ as indicated in the panel at lower left. The ranges for the spectral classes are given at the top (upper row for supergiants, lower row for giants), and at the bottom of the figure (for main-sequence stars)

lack of a significant outer convection zone. (In earlier-type stars of spectral type $\mathrm{O}$ and $\mathrm{B}$, shocks developing in unstable winds are the likely source of X-rays.)

MS stars define by far the largest stellar population for systematic survey studies (Maggio et al. 1987; Fleming et al. 1988; Schmitt et al. 1990a; Barbera et al. 1993). The ROSAT All-Sky Survey (RASS) from which the samples shown in Fig. 2 were drawn has contributed invaluably to our science by providing volume-limited samples including stars down to the end of the MS and to the minimum levels of X-ray activity. Comprehensive surveys of cool MS stars, some of them complete out to more than 10 pc, were presented by Schmitt et al. (1995), Fleming et al. (1995), Schmitt (1997) and 
Table 1. Symbols and units used throughout the text

\begin{tabular}{|c|c|}
\hline Symbol, acronym & Explanation \\
\hline$R_{*}$ & Stellar radius $[\mathrm{cm}]$ \\
\hline$R_{\odot}$ & Solar radius $\left[7 \times 10^{10} \mathrm{~cm}\right]$ \\
\hline$M_{\odot}$ & Solar mass $\left[2 \times 10^{33} \mathrm{~g}\right]$ \\
\hline$P_{\text {rot }}$ or $P$ & Rotation period $[\mathrm{d}]$ \\
\hline$p$ & Pressure $\left[\right.$ dyne $\left.\mathrm{cm}^{-2}\right]$ \\
\hline$L$ & Coronal loop semi-length $[\mathrm{cm}]$ \\
\hline$T$ & Coronal electron temperature $[\mathrm{K}]$ \\
\hline$n_{e}$ & Electron density $\left[\mathrm{cm}^{-3}\right]$ \\
\hline$n_{\mathrm{H}}$ & Hydrogen density $\left[\mathrm{cm}^{-3}\right]$ \\
\hline$B$ & Magnetic field strength $[\mathrm{G}]$ \\
\hline$f$ & Surface filling factor $[\%]$ \\
\hline$\Gamma$ & Loop area expansion factor (apex to base) \\
\hline$F_{X}$ & $\mathrm{X}$-ray surface flux $\left[\mathrm{erg} \mathrm{s}^{-1} \mathrm{~cm}^{-2}\right.$ ] \\
\hline$L_{X}$ & $\mathrm{X}$-ray luminosity $\left[\mathrm{erg} \mathrm{s}^{-1}\right]$ \\
\hline$L_{\text {bol }}$ & Stellar bolometric luminosity $\left[\mathrm{erg} \mathrm{s}^{-1}\right.$ ] \\
\hline$\Lambda=\Lambda_{0} T^{\gamma}$ & Cooling function $\left[\mathrm{erg} \mathrm{s}^{-1} \mathrm{~cm}^{3}\right]$ \\
\hline Ro & Rossby number \\
\hline RTV loop & Constant cross-section loop after Rosner et al. (1978) \\
\hline VAU loop & Expanding cross-section loop after Vesecky et al. (1979) \\
\hline 2-R flares & Two-Ribbon flares \\
\hline HRD & Hertzsprung-Russell Diagram \\
\hline EM & Emission Measure \\
\hline$Q, \mathrm{DEM}$ & Differential Emission Measure Distribution \\
\hline EMD & (discretized, binned) Emission Measure Distribution \\
\hline (ZA)MS & (Zero-Age) Main Sequence \\
\hline PMS & Pre-Main Sequence \\
\hline$(\mathrm{W}, \mathrm{C}) \mathrm{TTS}$ & (Weak-lined, classical) T Tauri Star \\
\hline $\mathrm{BD}$ & Brown Dwarf \\
\hline
\end{tabular}

Hünsch et al. (1999), with detection rates as high as 95\% per spectral class, except for the intrinsically faint or X-ray dark A stars. The survey sensitivities were sufficient to suggest a lower limit to the MS X-ray luminosity probably around a few times $10^{25} \mathrm{erg} \mathrm{s}^{-1}$ (Schmitt et al. 1995) which translates to a lower limit to the surface X-ray flux that is similar to that of solar coronal holes (Schmitt 1997).

Such studies have contributed much to our current understanding of coronal physics, in particular with regard to the dependence of magnetic activity on rotation, the ingredients controlling the coronal heating efficiency, and the feedback loop between activity and evolution, subjects broadly discussed across this review. Before moving on to giants and binaries, I now specifically address three fundamental issues within the main-sequence domain: that of very low-mass stars, brown dwarfs, and A stars with very shallow convective zones. 


\subsection{The coolest $M d$ warfs}

Beyond spectral type M5, the internal structure of dwarf stars changes significantly as they become fully convective. The classical $\alpha \omega$ dynamo can thus no longer operate. On the other hand, a distributed (or $\alpha^{2}$ ) dynamo may become relevant (e.g., Giampapa et al. 1996 and references therein). One would then naturally expect that both the magnetic flux on the surface and the topology of the magnetic fields in the corona systematically change across this transition, perhaps resulting in some discontinuities in the X-ray characteristics around spectral class dM5.

Observations do not seem to support this picture, however. The long-time lowestmass X-ray detection, VB 8 (M7e V) has shown steady emission at levels of $L_{X} \approx$ $10^{26} \mathrm{erg} \mathrm{s}^{-1}$ (Johnson 1981; Fleming et al. 1993; J. Drake et al. 1996) and flares up to an order of magnitude higher (Johnson 1987; Tagliaferri et al. 1990; J. Drake et al. 1996). If its X-ray luminosity $L_{X}$ or the ratio of $L_{X} / L_{\text {bol }}$ are compared with other late M dwarfs, a rather continuous trend becomes visible (Fleming et al. 1995, Fig. 3, although there have been scattered claims to the contrary, see, e.g., Barbera et al. 1993). The maximum levels attained by these stars $\left(L_{X} \approx 10^{-3} L_{\text {bol }}\right)$ remains the same across spectral class M. If a change from an $\alpha \omega$ to a distributive dynamo indeed does take place, then the efficiencies of both types of dynamos must be very similar, or the transition must be very

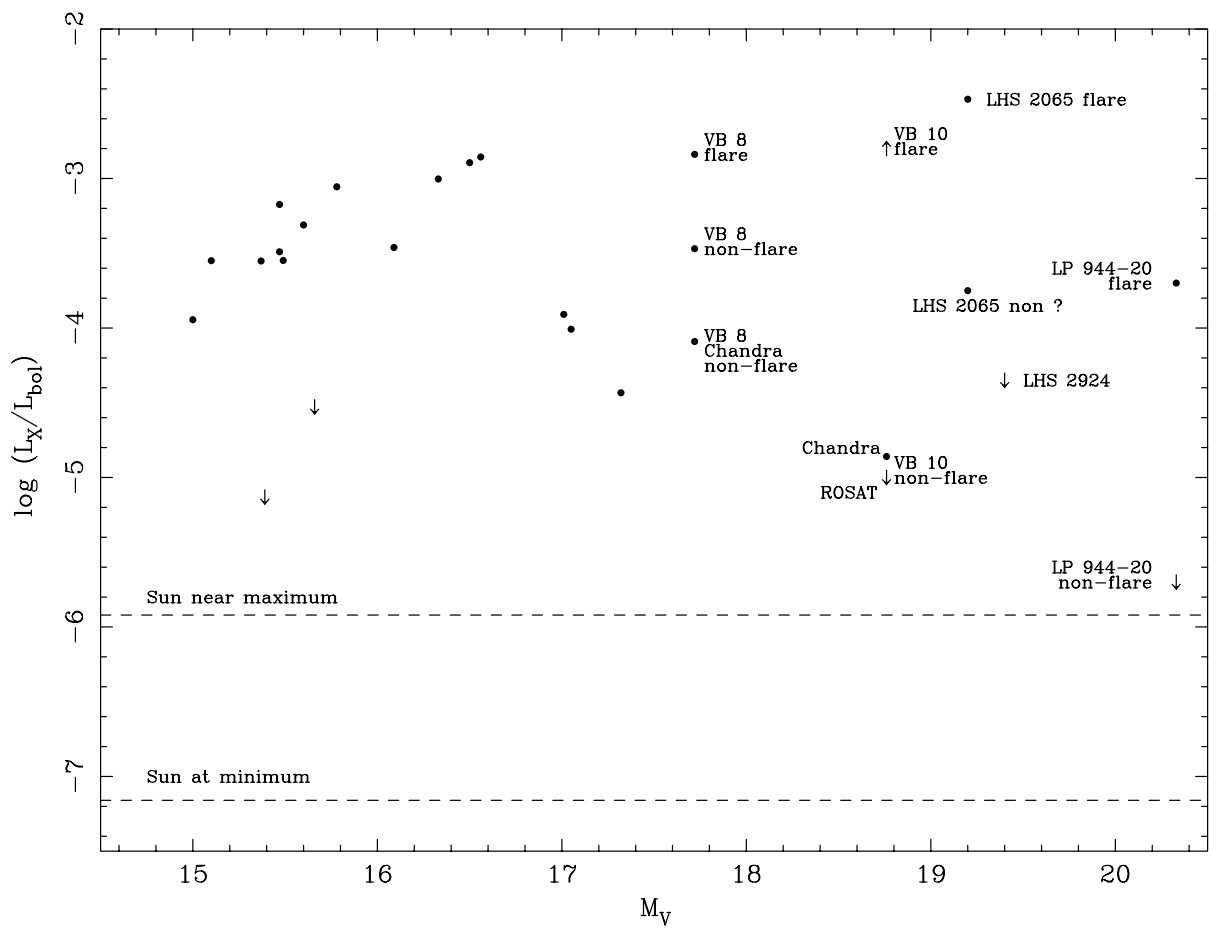

Fig. 3. Diagram showing $L_{X} / L_{\text {bol }}$ for lowest-mass stars later than spectral type M5. Flare and nonflaring values for several detections are marked (figure courtesy of T. Fleming and M. Giampapa, after Fleming et al. 2003) 
smooth and gradual, with the two regimes possibly overlapping (Fleming et al. 1993; Weiss 1993; J. Drake et al. 1996).

The same question also arose in the context of the coolest $\mathrm{M}$ dwarfs, namely relating to the boundary toward the substellar regime (around masses of $0.07 M_{\odot}$ ). A change in the magnetic behavior is suggested there, for the following reason. The photospheres of such stars are dominated by molecular hydrogen, with a very low ionization degree of approximately $10^{-7}$. Electric currents flow parallel to the coronal magnetic field lines in the predominant non-flaring force-free configuration, but since currents cannot flow into the almost neutral photosphere, any equilibrium coronal configuration will be potential, that is, not capable of liberating energy for heating (Fleming et al. 2000 and references therein). A precipitous drop of $L_{X}$ would thus be expected. Heating could be due to episodic instabilities in more complex magnetic configurations that then produce prominent flares. Indeed, stars at the bottom of the main-sequence (Fleming et al. 2000; Schmitt and Liefke 2002) and evolved brown dwarfs (see below) have been detected in X-rays during flares but not generally at steady levels, similar to what is seen in $\mathrm{H} \alpha$ observations (see references in Fleming et al. 2003). This picture has become somewhat questionable with the X-ray detection of VB 10 (M8e) during $3.5 \mathrm{hrs}$ at a level of $L_{X} \approx 2.4 \times 10^{25} \mathrm{erg} \mathrm{s}^{-1}$ and $\log L_{X} / L_{\text {bol }} \approx-4.9$ by Fleming et al. (2003) who claim this emission to be non-flaring. It is, however, inherently difficult to identify a steady process in data with very low signal-to-noise ratios (Sect. 13.3), so that the last word on the emission type in those stars may not have been spoken. A most productive strategy is to push the limit further toward lower-mass objects, as discussed below.

\subsection{Brown dwarfs (and planets?)}

Below the stellar mass limit at $0.07 M_{\odot}$, the realm of brown dwarfs (BD) has attracted immense attention in recent years. The field is, at the time of writing, still quite poorly explored in X-rays. Whereas young X-ray emitting BDs have now amply been detected in star forming regions, these objects behave like contracting $\mathrm{T}$ Tauri stars rather than evolved, low-mass MS stars. Recent findings on young BDs are therefore summarized in Sect. 18.3 in the context of star formation. At some quite early point in their evolution (before the age of $100 \mathrm{Myr}$ ), they must drop to quite low activity levels. Krishnamurthi et al. (2001) could not detect any BD in their Pleiades field down to a sensitivity limit of $L_{X} \approx 3 \times 10^{27} \mathrm{erg} \mathrm{s}^{-1}$.

First X-ray detections among older, contracted BDs now exist, but a number of anomalies come to light. The first X-ray detected evolved brown dwarf, LP 944-20, was recorded exclusively during a flare, with $L_{X} \approx 1.2 \times 10^{26} \mathrm{erg} \mathrm{s}^{-1}$ and with a decay time of $\approx 5400 \mathrm{~s}$ (Rutledge et al. 2000). Although this star is old $(\approx 500 \mathrm{Myr})$, it has not spun down ( $P_{\text {rot }} \leq 4.4 \mathrm{hrs}$ ). While it should thus be in a "supersaturated" regime (Sect. 5), it shows no detectable steady X-ray emission, quite in contrast to its strong and also flaring radio radiation (Berger 2002). Tsuboi et al. (2003) found the BD companion of TWA 5 (with an age of $12 \mathrm{Myr}$ ) at levels of $L_{X} \approx 4 \times 10^{27} \mathrm{erg} \mathrm{s}^{-1}$ and, again, below the empirical saturation limit, with a rather soft spectrum $(k T=0.3 \mathrm{keV})$. Finally, Briggs and Pye (2004) reported a weak detection of Roque 14 in the Pleiades (age $100 \mathrm{Myr}$ ), with $L_{X} \approx 3 \times 10^{27} \mathrm{erg} \mathrm{s}^{-1}$. This time, the emission is not compatible with a flare if the decay time is shorter than $4 \mathrm{ks}$. 
Ultimately, X-ray observations offer the possibility to detect (X-ray dark) planets as they eclipse part of the corona of their parent star while in transit. Such methods are particularly promising for low-mass stars as a Jupiter-like planet could eclipse a rather significant coronal area. Briggs and Pye (2003) presented an example of a Pleiades member star that showed a significant dip in a flaring light curve (Sect. 11.13). The requirements for such a flare eclipse to occur are somewhat implausible, however, unless enhanced magnetic activity is induced by star-planet interactions (see Briggs and Pye 2003).

\subsection{A-type stars}

The outer convection zones of stars become very shallow toward early $\mathrm{F}$ stars and disappear in A-type dwarfs. These stars are therefore not capable of operating a classical $\alpha \omega$-type dynamo. Even if magnetic fields existed in early A-type stars, efficient coronal energy release is not expected because no strong surface convective motions are present to transport energy into non-potential coronal fields. Coincidentally, the acoustic flux from the interior reaches a maximum for late A and early F stars (Vaiana et al. 1981, Schrijver 1993 and references therein), a fact that has provoked several early survey programs to look for strong X-ray emission in these stars (Vaiana et al. 1981; Pallavicini et al. 1981; Topka et al. 1982; Walter 1983; Schmitt et al. 1985a). Acoustic heating has meanwhile been widely ruled out as the principal coronal heating mechanism along the main sequence (Vaiana et al. 1981; Stern et al. 1981, although the issue re-surfaces from time to time, e.g., Mullan and Cheng 1994a and Mullan and Fleming 1996, for M dwarfs). However, a significant "base" contribution of acoustic waves in particular to the heating of the lower atmospheres of A-F stars remains a viable possibility (Schrijver 1993; Mullan and Cheng 1994b).

Investigations of magnetic activity in A-type stars have proceeded along three principal lines: i) Search for genuine magnetic activity in single, normal A-type and early F stars; ii) study of magnetic activity in chemically peculiar Ap/Bp stars; iii) search for signatures of magnetic fields in very young, forming A-type stars (e.g., Herbig Ae/Be stars). Some selected results are briefly summarized below.

\subsubsection{X-ray emission from normal A stars}

Volume-limited stellar samples reveal a rather abrupt onset of X-ray emission around spectral type A7-F0, with a large range of luminosities developing across spectral class F (Schmitt 1997). The drop in X-rays toward earlier stars is appreciable: The definitive X-ray detection of Altair (A7 V) shows a very soft spectrum at low luminosity $\left(\log L_{X} \approx\right.$ $27.1, \log L_{X} / L_{\text {bol }} \approx-7.5$, Schmitt et al. 1985a), with a temperature of only $\approx 1 \mathrm{MK}$ (Golub et al. 1983; Schmitt et al. 1990a). Quite in general, ostensibly single A-to-early F stars show distinctly soft spectra if detected in X-rays (Panzera et al. 1999).

Optically selected samples have produced a number of additional detections up to early A stars, among them quite luminous examples. However, there are several reasons to believe that unidentified cooler companions are responsible for the X-rays. Given the rapid evolution of A-type stars, a companion of, say, spectral type $\mathrm{K}$ or M would still be quite active. The companion hypothesis is thus particularly likely for X-ray 
luminous examples with a hard spectrum (Golub et al. 1983). Late-B and A-type stars in the Pleiades, for example, have X-ray properties that are indeed consistent with the presence of a cooler coronal companion (Daniel et al. 2002; Briggs and Pye 2003). Another indication is the break-down of any correlation between rotation and $L_{X}$ or rotation and $B-V$ among these stars, once again indicating that the X-rays may not actually be related to the A star (Simon et al. 1995; Panzera et al. 1999), although F stars are considered to be coronal sources in any case (see below).

Illustrative examples of the complications involved in A-star X-ray astronomy are nearby A-star binaries. The X-ray detections of the A-type binary Castor, even as a strong flaring source (Pallavicini et al. 1990b; Schmitt et al. 1994b; Gotthelf et al. 1994), opened up new speculations on A-star coronae. Spatially resolved observations (Güdel et al. 2001a; Stelzer and Burwitz 2003) with XMM-Newton and Chandra showed that both components are X-ray active. However, Castor is in fact a hierarchical quadruple system consisting of two A stars that are each surrounded by a low-mass (K-M type, Güdel et al. 2001a and references therein) companion. They are additionally accompanied by the well-known X-ray strong M-dwarf binary YY Gem. Both Castor components are frequently flaring (Güdel et al. 2001a; Stelzer and Burwitz 2003) and reveal X-ray spectra and fluxes that are quite similar to M dwarfs.

Restricting our attention now to the few genuine late A- or early F-type coronal emitters, we find that their weak dynamo operation is generally not able to brake the rapidly spinning star considerably during their short lifetime (Schmitt et al. 1985a). One step further, several authors (Pallavicini et al. 1981; Walter 1983; Simon and Landsman 1991) have questioned the presence of any activity-rotation relation from spectral class A to F5 (beyond which it holds) altogether. These coronae are also conspicuous by their severe deficit of X-ray emission compared to chromospheric and transition region fluxes; the latter can be followed up to mid-A type stars at quite high levels (Simon and Drake 1989, 1993; Simon and Landsman 1991, 1997). Whether or not these atmospheres are indeed heated acoustically and drive an "expanding", weak and cool corona (Simon and Drake 1989) or whether they are heated magnetically, the X-ray deficit and the low coronal temperatures clearly attest to the inability of these stars to maintain substantial, hot coronae in any way comparable to cooler active stars, their appreciable chromospheres notwithstanding.

\subsubsection{Chemically peculiar A stars}

Magnetic chemically peculiar stars of spectral type Bp or Ap are appreciable magnetic radio sources (Drake et al. 1987), but they have produced quite mixed results in X-rays. While a number of detections were reported early on (Cash et al. 1979b; Cash and Snow 1982; Golub et al. 1983), most Bp/Ap stars remained undetected, and only few of them can be identified as probably single stars (S. Drake et al. 1994b). When detected, their X-ray luminosities are quite high $\left(\log L_{X} \approx 29.5-30, \log L_{X} / L_{\text {bol }} \approx-6\right)$ and do not follow the systematics of earlier-type stars. Given the strong surface magnetic fields in Bp/Ap stars, the currently favored models involve dipolar magnetospheres either featuring equatorial reconnection zones that heat plasma (S. Drake et al. 1994b) or winds that are magnetically guided to the equatorial plane where they collide and heat up (Babel and Montmerle 1997). 
A suspiciously high detection rate of CP stars was reported for the open cluster NGC 2516 by Dachs and Hummel (1996), Jeffries et al. (1997), and Damiani et al. (2003) (6 or 7 detected out of 8 observed CP stars in the latter study, amounting to one half of all detected stars optically identified as A-type). Such statistical samples may argue in favor of some of the Ap stars in fact being the sites of the X-ray emission. As for nonmagnetic Am stars, only scattered evidence is present that some may be X-ray sources (Randich et al. 1996b; Panzera et al. 1999), but again the caveats with undetected cooler companions apply.

\subsubsection{Herbig Ae/Be stars}

The nature of strong X-ray emission from pre-main sequence Herbig Ae/Be stars has remained rather controversial. Models include unstable stellar winds, colliding winds, magnetic coronae, disk coronae, wind-fed magnetospheres, accretion shocks, the operation of a shear dynamo, and the presence of unknown late-type companions. Some X-ray properties are reminiscent of hot stars (Zinnecker and Preibisch 1994), others point to coronal activity as in cool stars, in particular the presence of flares (Hamaguchi et al. 2000; Giardino et al. 2004) and very high temperatures $(T \gtrsim 35 \mathrm{MK}$, Skinner et al. 2004). For reviews - with differing conclusions - I refer the reader to the extensive critical discussions in Zinnecker and Preibisch (1994), Skinner and Yamauchi (1996), and Skinner et al. (2004).

\subsection{Giants and supergiants}

The evolution of X-ray emission changes appreciably in the domain of giants and supergiants. The area of red giants has attracted particular attention because hardly any $\mathrm{X}$-rays are found there. The cause of the X-ray deficiency is unclear. It may involve a turn-off of the dynamo, a suppression by competing wind production, or simply strong attenuation by an overlying thick chromosphere (Sect. 17.3). This region of the HRD was comprehensively surveyed by Maggio et al. (1990) using Einstein, and by Ayres et al. (1995), Hünsch et al. (1998a), and Hünsch et al. (1998b) using the ROSAT AllSky Survey. A volume-limited sample was discussed by Hünsch et al. (1996). Several competing effects influence the dynamo during this evolutionary phase especially for stars with masses $\gtrsim 2 M_{\odot}$ : While the growing convection zone enhances the dynamo efficiency, angular momentum loss via a magnetized wind tends to dampen the dynamo evolution. These processes occur during the rather rapid crossing of the Hertzsprung gap toward $\mathrm{M}$ type giants and supergiants. The systematics of the X-ray emission are still not fully understood.

A rather exquisite but very small family of stars is defined by the so-called FK Com stars, giants of spectral type $\mathrm{K}$ with an unusually rapid rotation and signs of extreme activity. Their X-ray coronae are among the most luminous $\left(L_{X} \gtrsim 10^{32} \mathrm{erg} \mathrm{s}^{-1}\right)$ and hottest known (with dominant temperatures up to 40 MK; e.g., Welty and Ramsey 1994; Gondoin et al. 2002; Gondoin 2004b; Audard et al. 2004). These stars are probably descendants of rapidly rotating B-A MS stars that, during the fast evolution across the Hertzsprung gap, have been able to maintain their rapid rotation as the convection zone 
deepened, while now being in a stage of strong magnetic braking due to increased magnetic activity (Gondoin et al. 2002; Gondoin 2003a). The leading hypothesis, however, involves a merger of a close binary system, in which the orbital angular momentum of the companion is transferred to the primary (Bopp and Stencel 1981).

\subsection{Close binaries}

Close, tidally interacting binaries keep their fast rotation rates often throughout their MS life and possibly into the subgiant and giant evolution. Their rotation-induced dynamos maintain high magnetic activity levels throughout their lifetimes, making them ideal laboratories for the study of magnetic dynamo theory.

The most common binary systems available for study are RS CVn-type systems that typically contain a G- or K-type giant or subgiant with a late-type subgiant or MS companion. The similar class of BY Dra-type binaries contain two late-type MS stars instead. If their separation is sufficiently small, the two components may come into physical contact, defining the class of W UMa-type contact systems (see Sect. 4.7 below). And finally, Algol-type binaries are similar to RS CVn systems, but the MS component is of early type (typically a B star). The cool subgiant fills its Roche lobe, and mass transfer may be possible.

Extensive X-ray surveys of RS CVn-type binaries were presented by Walter and Bowyer (1981), Drake et al. (1989), Drake et al. (1992), Dempsey et al. (1993a), Dempsey et al. (1993b), and Fox et al. (1994). Comparative studies suggested that the secondary star plays no role in determining the activity level of the system other than providing the mechanism to maintain rapid rotation (Dempsey et al. 1993a). However, the surface $\mathrm{X}$-ray activity does seem to be enhanced compared to single stars with the same rotation period (Dempsey et al. 1993a). The X-ray characteristics of BY Dra binaries are essentially indistinguishable from RS CVn binaries so that they form a single population for statistical studies (Dempsey et al. 1997).

The X-ray emission of Algols was surveyed by White and Marshall (1983), McCluskey and Kondo (1984) and Singh et al. (1995). White et al. (1980) were the first to indicate that X-rays from Algol-type binaries are also coronal (with X-ray sources located on the late-type secondary), and that they resemble RS CVn-type binaries in that respect. However, Algols are underluminous by a factor of 3-4 compared to similar RS CVn binaries (Singh et al. 1996b) . It is therefore rather unlikely that possible accretion streams contribute significantly to the X-ray emission in Algol.

Ottmann et al. (1997) presented the first survey of Population II binaries. They concluded that their overall X-ray emission is weaker than what is typical for similar Pop I RS CVn binaries. Here, part of the trend may, however, be explained by the Pop II sample containing fewer evolved stars. On the other hand, the reduced metallicity may also inhibit efficient coronal radiation.

\subsection{Contact binary systems}

W UMa systems are contact binaries of spectral type F-K with rotation periods from $0.1-1.5 \mathrm{~d}$. They were first detected in X-rays by Caroll et al. (1980) and surveyed by 
Cruddace and Dupree (1984). Their rapid rotation periods suggest enhanced activity, and this is indeed confirmed by more recent comprehensive surveys (Stępieǹ et al. 2001). However, early work already found an order-of-magnitude deficiency in $L_{X} / L_{\text {bol }}$ when compared to similar detached systems (Cruddace and Dupree 1984; Vilhu and Rucinski 1983; Vilhu 1984). This phenomenon is also known as "supersaturation" (see Sect. 5). A survey by McGale et al. (1996) with ROSAT essentially confirmed the luminosity deficit in all targets and additionally reported somewhat lower maximum temperatures and a smaller amount of very hot plasma when compared with detached RS CVn binaries.

The related near-contact binaries do not share a common envelope but may in fact be evolutionary precursors of contact systems. They were studied by Shaw et al. (1996) who found luminosities similar to those of contact systems but again significantly lower than those of RS CVn binaries.

\section{X-ray activity and rotation}

\subsection{Rotation-activity laws}

Stellar rotation and magnetic activity operate in a feedback loop; as a single low-mass MS star ages, it sheds a magnetized wind, thus spinning down due to angular momentum transport away from the star. This, in turn, weakens the internal dynamo and thus reduces magnetic activity (e.g., Skumanich 1972). This negative feedback loop tends to converge toward a definitive rotation period $P$ that depends only on mass and age once the star has evolved for a few $100 \mathrm{Myr}$ (e.g., Soderblom et al. 1993). It is thus most likely rotation, and only indirectly age, that determines the level of magnetic activity, a contention confirmed in recent studies by Hempelmann et al. (1995). X-rays offer an ideal and sensitive tool to test these dependencies, and corresponding results were found from the initial Xray survey with Einstein. Pallavicini et al. (1981) suggested a relation between X-ray luminosity and projected rotational velocities $v \sin i$ (where $v$ is measured in $\mathrm{km} \mathrm{s}^{-1}$ )

$$
L_{X} \approx 10^{27}(v \sin i)^{2} \quad\left[\mathrm{erg} \mathrm{s}^{-1}\right]
$$

(although the stellar sample included saturated stars, which were recognized only later). A similar trend was visible in a sample shown by Ayres and Linsky (1980). The overall relation was subsequently widely confirmed, e.g., by Maggio et al. (1987) for F-G MS and subgiant stars, or by Wood et al. (1994) for a large sample of nearby stars based on EUV measurements (with somewhat smaller indices of $1.4-1.6$ ). For the surface flux $F_{X}$ or the ratio $L_{X} / L_{\text {bol }}$, a relation like (1) implies

$$
F_{X}, \frac{L_{X}}{L_{\text {bol }}} \propto \Omega^{2} \propto P^{-2}
$$

where $\Omega$ is the angular rotation velocity and we have, for the moment, ignored the photospheric-temperature term distinguishing the two measures on the left-hand side. Walter (1981) reported $L_{X} / L_{\text {bol }} \propto \Omega$ but his sample included saturated stars (not recognized as such at that time). He later introduced broken power-laws and thus in fact corrected for a saturation effect in rapid rotators (Walter 1982, see below). Schrijver et al. (1984) included other determining factors, concluding, from a common-factor analysis, 
that the specific emission measure $\zeta$ (total EM divided by the stellar surface area) is related to $P$ and the (dominant) coronal temperature as

$$
\zeta=10^{28.6 \pm 0.2} T^{1.51 \pm 0.16} P^{-0.88 \pm 0.14}
$$

where here $T$ is given in MK, $P$ in d, and $\zeta$ in $\mathrm{cm}^{-5}$. Since the dynamo efficiency also depends on the convection zone depth, Noyes et al. (1984) and Mangeney and Praderie (1984) introduced the Rossby number $R o$ as the ratio of the two relevant time scales of rotation and convection ( $R o=P / \tau_{c}$, where $\tau_{c}$ is the convective turnover time). The most general rotation-activity diagrams that may include stars of various spectral classes and radii are now conventionally drawn for the variables $R o$ and $L_{X} / L_{\text {bol }}$ (Dobson and Radick 1989) although there has been considerable discussion as to which parameters are to be preferred (Rutten and Schrijver 1987; Basri 1987). A critical appraisal of the use of $R o$ for activity-rotation relations was given, for example, by Stępieǹ (1994) who described some limitations also with regard to the underlying theoretical concepts.

The overall rotation-activity relation was perhaps best clarified by using large samples of stars from stellar clusters. The comprehensive diagram in Fig. 4 clearly shows a regime where $L_{X} / L_{\text {bol }} \propto R o^{-2}$ for intermediate and slow rotators (from Randich et al. 2000). However, in fast rotators $L_{X}$ appears to become a unique function of $L_{\mathrm{bol}}$, $L_{X} / L_{\text {bol }} \approx 10^{-3}$ regardless of the rotation period (Agrawal et al. 1986a; Fleming et al. 1988; Pallavicini et al. 1990a). The tendency for a corona to "saturate" at this level once the rotation period (or the Rossby number) is sufficiently small, or $v$ sufficiently large, was identified and described in detail by Vilhu and Rucinski (1983), Vilhu (1984), Vilhu and Walter (1987), and Fleming et al. (1989). It is valid for all classes of stars but the onset of saturation varies somewhat depending on the spectral type. Once MS coronae are saturated, $L_{X}$ also becomes a function of mass, color, or radius simply owing to the fundamental properties of MS stars.

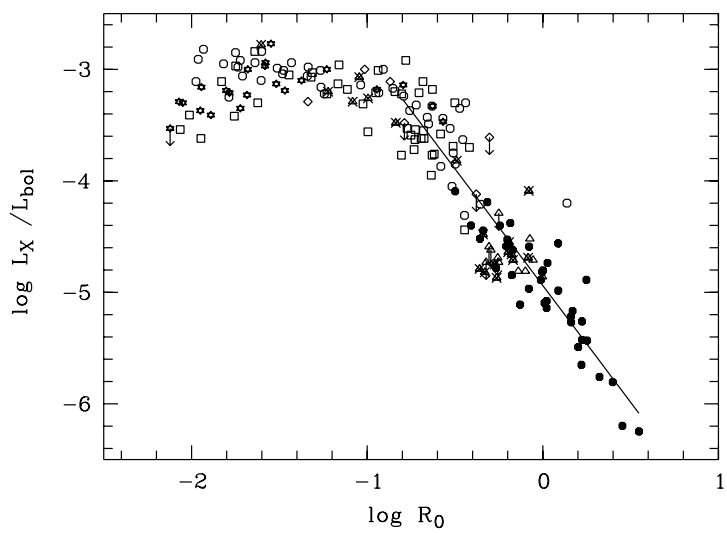

Fig. 4. Activity-rotation relationship compiled from several samples of open cluster stars. Key to the symbols: circles: Pleiades; squares: IC 2602 and IC 2391: stars: $\alpha$ Per; triangles: single Hyades stars; crossed triangles: Hyades binaries; diamonds: IC 4665; filled symbols: field stars (figure courtesy of S. Randich, after Randich et al. 2000, by the kind permission of the Astronomical Society of the Pacific) 


\subsection{Activity and rotation in stars with shallow convection zones}

The shallow convection zones toward early $\mathrm{F}$ stars express themselves through two effects. First, the maximum $L_{X} / L_{\text {bol }}$ ratio or the maximum surface flux $F_{X}$ decrease when compared to the nearly constant values for cooler stars (Walter 1983; Vilhu and Walter 1987; Gagné et al. 1995b, also Wood et al. 1994 for EUV emission). In other words, the dynamo becomes less efficient. And second, little or no dependence of $L_{X}$ on rotational parameters is found in early F stars (Pallavicini et al. 1981; Walter 1983; Simon and Drake 1989, 1993; Stauffer et al. 1994). These results were initially taken to suggest a change in the dynamo mode (possibly from non-solar-like to solar-like at a spectral type of $\approx F 5 \mathrm{~V}$ ), or a change in the surface magnetic field configuration as the convection zone deepens (Schrijver and Haisch 1996), or a change from acoustic to magnetic coronal heating (Simon and Drake 1989).

Comprehensive surveys by Mangeney and Praderie (1984), Schmitt et al. (1985a), and Dobson and Radick (1989), however, put the absence of an activity-rotation relation into question. If activity is correlated with the Rossby number, then in fact the same dependence is recovered for early F-type stars as for later stars. Consideration of the convective turnover time may indeed be important for these stars because, in contrast to the range of cooler stars, $\tau_{c}$ varies largely across the F spectral class (e.g., Stauffer et al. 1994; Randich et al. 1996a). Once a "basal" flux is subtracted from the observed chromospheric flux, one also finds that all convective stars, including late-A and F stars, follow the same coronal-chromospheric flux-flux relations (Sect. 6; Schrijver 1993). It is essentially the dynamo efficiency (i.e., the surface magnetic flux density for a given rotation rate) that decreases toward earlier F stars while the basic dynamo physics may be identical in all convective main-sequence stars (except, possibly, for late-M dwarfs; Sect. 4.2). At the same time, the magnetic braking efficiency is reduced in early-F stars. The dynamo and, as a consequence, the magnetic flux production are never sufficient in these stars to "saturate" the way cooler stars do.

\subsection{Rotation and saturation; supersaturation}

The issue of which parameters most favorably represent the activity-rotation relation was studied in great detail by Pizzolato et al. (2003) for MS stars in the context of saturation. They reported the following results (see also Micela et al. 1999a for a qualitative description): i) The rotation period is a good activity indicator for non-saturated stars for which it correlates with the unnormalized $L_{X}$ regardless of mass. The slope of the power law is -2 . ii) The period at which saturation is reached increases with decreasing mass $\left(\approx 1.5 \mathrm{~d}\right.$ for an $1.05 M_{\odot}$ star, $\approx 3.5 \mathrm{~d}$ for a $0.7 M_{\odot}$ star, and rapidly increasing further for lower masses), therefore reaching to progressively lower maximum $L_{X}$. iii) In the saturation regime, $L_{X} / L_{\text {bol }}$ becomes strictly independent of rotation and mass with the possible exception of stars with masses $>1.1 M_{\odot}$. iv) A modified, empirical convective turnover time (hence a modified Rossby number $R o^{\prime}$ ) can be derived as a function of stellar mass with the goal of defining a universal function $L_{X} / L_{\text {bol }}=f\left(R o^{\prime}\right)$ that is valid for all cool stars irrespective of mass. The empirical turnover time is found to be similar to the calculated $\tau_{c}$, and it scales with $L_{\mathrm{bol}}^{-1 / 2}$. As a consequence, the two descriptions, $L_{X}$ vs. $P$ and $L_{X} / L_{\text {bol }}$ vs. $R o^{\prime}$ become fully equivalent for non-saturated stars. v) 
The saturation is triggered at a fixed $R o^{\prime} \approx 0.1$, and the critical period where saturation starts is $P_{\text {sat }}$. The full description of the rotation-activity relation in this picture is, then,

$$
\left.\begin{array}{ll}
\frac{L_{X}}{L_{\mathrm{bol}}} \propto R o^{\prime-2} \text { and } L_{X} \propto P^{-2} & \text { for } \quad P \gtrsim \\
\frac{L_{X}}{L_{\mathrm{bol}}} \approx 10^{-3} & \text { for } P \lesssim
\end{array}\right\} P_{\text {sat }} \approx 1.2\left(\frac{L_{\mathrm{bol}}}{L_{\odot}}\right)^{-1 / 2}
$$

As rotational equatorial velocities exceed $\approx 100 \mathrm{~km} \mathrm{~s}^{-1}$, the $L_{X} / L_{\text {bol }}$ values begin to slightly decrease again. This "supersaturation" phenomenon (Prosser et al. 1996; Randich et al. 1996a; James et al. 2000, Fig. 4) may be ascribed to a fundamental change of the dynamo action or to a decrease of the surface coverage with active regions (see Sect. 5.4).

\subsection{Physical causes for saturation and supersaturation}

Considering all aspects described above, it seems fair to say that all spectral classes between $\mathrm{F}$ and $\mathrm{M}$ are capable of maintaining coronae up to a limit of $\log \left(L_{X} / L_{\text {bol }}\right) \approx$ -3.0. Some decrease of the maximum ("saturation") level toward earlier F and late-A stars may be real because of the shallowness of the convection zone in these stars. The physical causes of saturation and supersaturation are not well understood. Ideas include the following:

1. The internal dynamo saturates, i.e., it produces no more magnetic flux if the rotation period increases (Gilman 1983; Vilhu and Walter 1987).

2. The surface filling factor of magnetic flux approaches unity at saturation (Vilhu 1984). This is also motivated by a strong correlation between saturated $L_{X}$ and radius rather than $L_{X}$ and surface temperature (Fleming et al. 1989). However, if the entire solar surface were filled with normal active regions, its X-ray luminosity would amount to only $\approx(2-3) \times 10^{29} \mathrm{erg} \mathrm{s}^{-1}$ (Vaiana and Rosner 1978; Wood et al. 1994), with $L_{X} / L_{\text {bol }} \approx 10^{-4}$ (Vilhu 1984), short of the empirical saturation value by one order of magnitude. To make up for this deficiency, one requires enhanced densities, larger coronal heights, or different mechanisms such as continuous flaring (see Sect. 13). The detection of rotational modulation in some saturated or nearly saturated stars (Güdel et al. 1995; Kürster et al. 1997; Audard et al. 2001a, see Sect.11.12) casts some doubt on a stellar surface that is completely covered with an ensemble of similar active regions.

3. Jardine and Unruh (1999) argued that the radius where centrifugal forces balance gravity (the co-rotation radius) approaches the outer X-ray coronal radius in rapid rotators. As the rotation rate increases, centrifugal forces lead to a rise in pressure in the outer parts of the largest loops. Once the co-rotation radius drops inside the corona, the local gas pressure may increase sufficiently to blow open the magnetic structures, thus leading to open, X-ray dark volumes. This mechanism confines the coronal height. This coronal "stripping" overcomes effects due to increased pressure, leading to approximately constant emission in the saturation regime. As the rotation rate increases further and the corona shrinks, a more structured low corona is 
left behind that is less luminous ("supersaturated") and is more prone to rotational modulation (Jardine 2004). Deep rotational modulation has indeed been found in the supersaturated young G star VXR45 (Marino et al. 2003a, see Sect. 11.12).

4. An alternative explanation was given by Stępieǹ et al. (2001) who conjectured that rapid rotation produces, through a strong centrifugally induced gradient of the effective gravity from the equator to the pole, a heat flux excess toward the poles in the stellar interior. Consequently, an excess convective updraft develops at the poles, accompanied by poleward circulation flows in the lower part of the convection zone, and equatorward surface return flows. This circulation system sweeps magnetic fields from the generation region in the lower convection zone toward the poles. This effect strongly amplifies with rotation rate, thus leaving progressively more of the equatorial region free of strong magnetic fields. Therefore, the filling factor decreases. The effect is particularly strong in W UMa-type contact binaries although an additional suppression, presumably due to equatorial flows between the components, is found. The suppression of equatorial activity has an interesting side effect in that loss of angular momentum through a wind is strongly suppressed (Stępieǹ et al. 2001).

\subsection{Rotation and activity in pre-main sequence stars, giants and binaries}

Among giants and supergiants, the dependence between rotation and activity becomes much less evident (Maggio et al. 1990). Whereas cooler giants follow the same dependence as MS stars, this does not hold for warmer giants (Ayres et al. 1998). The evolution across the Hertzsprung gap features two competing effects, namely a deepening convection zone that strengthens the dynamo, and rapid spin-down that weakens it. It is likely that the rapid evolution through this regime does not leave sufficient time for the stars to converge to a unified rotation-activity relation.

There are also mixed results from pre-main sequence stars. Whereas the standard behavior including saturation applies to some star-forming regions such as Taurus, other regions show all stars in a saturation regime, up to rotation periods of $30 \mathrm{~d}$. This effect could be related to the long convective turnover time in these stars, as discussed by Flaccomio et al. (2003c) and Feigelson et al. (2003) (see Sect. 18.1 for further details).

Close binary systems are interesting objects to study the effect of rapid rotation that is maintained due to tidal interactions with the orbiting companion. Walter and Bowyer (1981) found

$$
\frac{L_{X}}{L_{\text {bol }}} \propto \Omega
$$

(and no dependence on $v$ ) although this relation contains much scatter and may in fact be a consequence of a relation between stellar radius and orbital period in close binaries, larger stars typically being components of longer-period systems and being bolometrically brighter (Walter and Bowyer 1981; Rengarajan and Verma 1983; Majer et al. 1986; Dempsey et al. 1993a); this explanation was however questioned again by Dempsey et al. (1997). In general, caution is in order with regard to activity-rotation relationships in these binaries because many of them are at or close to the saturation limit. 


\section{Flux-flux relations}

\subsection{Chromosphere-transition region-corona}

Flux-flux (or luminosity-luminosity) relations from the chromosphere to the corona contain telltale signatures of the overall heating process and of systematic deficiencies at any of the temperature layers. The standard relation between normalized luminosities from transition-region emission lines such as C IV and coronal X-ray luminosities is non-linear, with a power-law slope of about 1.4 - 1.5 (Ayres et al. 1981b; Vilhu 1984; Agrawal et al. 1986a; Haisch et al. 1990c; Ayres et al. 1995); the power-law becomes steeper if chromospheric lines are used, e.g., $\mathrm{Mg}$ II; thus

$$
\frac{L_{X}}{L_{\text {bol }}} \approx\left(\frac{L_{\mathrm{C} \text { IV }}}{L_{\text {bol }}}\right)^{1.5} ; \quad \frac{L_{X}}{L_{\text {bol }}} \approx\left(\frac{L_{\mathrm{Mg} \text { II }}}{L_{\text {bol }}}\right)^{3}
$$

(see Fig. 5). These relations hold for RS CVn-type binaries as well (Dempsey et al. 1993a), although Mathioudakis and Doyle (1989) reported a near-linear correlation between the Mg II and and X-ray surface fluxes in dMe and dKe dwarfs. Schrijver and co-workers suggested that a color-dependent basal component be subtracted from the chromospheric (and partly transition region) stellar line fluxes to obtain the magnetically induced excess flux $\Delta_{H K}$ in the Ca II H\&K lines (Schrijver 1983, 1987; Schrijver

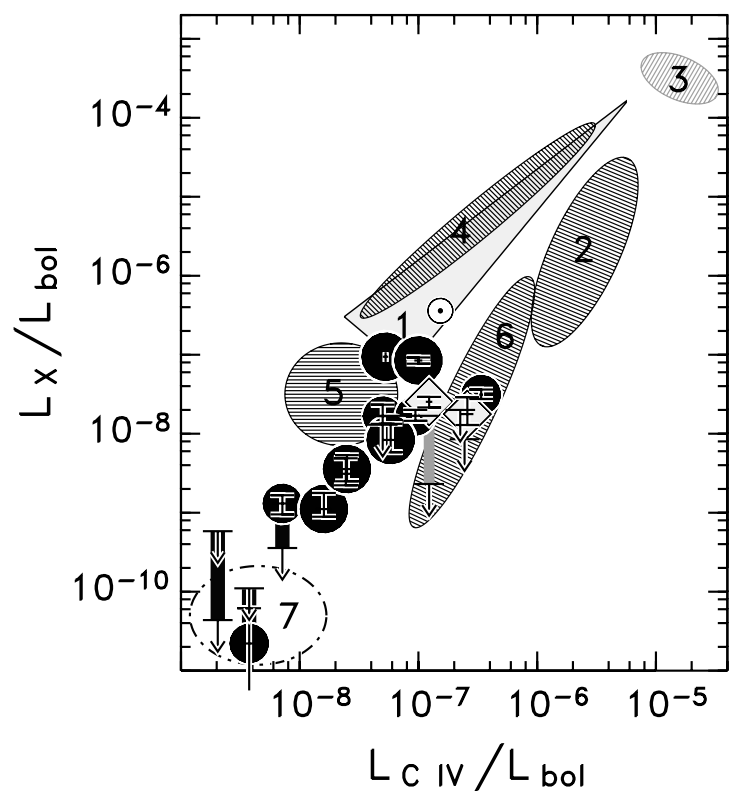

Fig. 5. "Flux-flux" diagram for $L_{X}$ and the C IV luminosity. Different groups of stars are schematically represented by different shading, labeled as follows: 1 standard relation for main-sequence stars; 2 X-ray deficient G supergiants; 3, 4 G-K0 III giants; 5 low-activity K0-1 III giants, 6 X-ray deficient F-G0 Hertzsprung gap stars; 7 probable region of red giants (Aldebaran, Arcturus); the black circles mark "hybrid" stars (figure courtesy of T. Ayres, after Ayres 2004) 
et al. 1992; Rutten et al. 1991 and further references cited therein). The justification for this procedure is that the flux-flux power-law relations tighten and become colorindependent. They flatten somewhat but remain non-linear between X-rays and chromo-

spheric fluxes: $F_{X} \propto \Delta_{H K}^{1.5-1.7}$. On the other hand, this procedure results in little change for the X-ray vs. transition region flux correlation (Rutten et al. 1991; Ayres et al. 1995). The basal chromospheric flux is then independent of activity and was suggested to be the result of steady (non-magnetic) acoustic heating (Schrijver 1987). No such basal flux is found for X-rays (Rutten et al. 1991) which may imply that any lower limit to the average $\mathrm{X}$-ray surface flux may be ascribed to a genuine minimum magnetic heating. If done so, the lower limit to the X-ray surface flux empirically found by Schmitt (1997) implies, however, a minimum magnetic chromospheric flux that is still much in excess of the basal fluxes for G-M stars, thus putting into question whether truly basal stars are realized, except possibly for F-type stars (see also Dempsey et al. 1997).

The flux-flux relations must be rooted in the magnetic flux on the stellar surface. Whereas chromospheric fluxes appear to depend non-linearly on the average photospheric magnetic flux density $f B$ ( $f$ is the surface filling factor of the magnetic fields) both for solar features and for entire stars (Schrijver et al. 1989a; Schrijver and Harvey 1989), the stellar coronal correlation between X-ray surface flux and $f B$ becomes nearly linear (Schrijver et al. 1989a). This suggests that an important cause of the non-linearities in the chromospheric-coronal flux-flux correlations is actually rooted in the behavior of the chromospheric radiative losses. On the other hand, Ayres et al. (1996) explained the non-linearity in the corona-transition region flux-flux relation by the increasing coronal temperatures with increasing activity (Sect. 9.5), bringing a progressively larger fraction of the emission into the X-ray band.

For various solar features (active regions, bright points, etc), a strong linear correlation was reported between $L_{X}$ and total unsigned magnetic flux (Fisher et al. 1998); the principal determining factor is the surface area of the feature. Interestingly, this correlation extends linearly over orders of magnitude to magnetically active stars. Because the entire stellar coronae may be made up of various solar-like features, the overall correlation suggests that a common heating mechanism is present for all solar and stellar coronal structures (Pevtsov et al. 2003). Empirically, a somewhat different relation was suggested by Stępieǹ (1994) if the stellar color (or the photospheric effective temperature) was also taken into account, namely $F_{X} \propto T^{8.3} B^{1.9}$ where $B$ is the surface magnetic field strength.

There are interesting deviations from the flux-flux correlations, in particular in the giant and supergiant domain. These are briefly addressed in the context of evolution and coronal structure in Sect. 17.2 and 17.4. Also, several flux-flux relations have been reported between flaring and low-level emission. I describe those in Sect. 13.1.

\subsection{Radio - X-ray correlations}

There is considerable interest in correlating emissions that connect different parts of causal chains. As I will discuss further in Sect.12.2, standard flare models involve electron beams (visible at radio wavelengths) that heat the chromospheric plasma to $\mathrm{X}$-ray emitting temperatures. The heating processes of the quiescent coronal plasma 
may be entirely different, however. Nevertheless, average radio and X-ray luminosities are nearly linearly correlated in magnetically active stars (Güdel and Benz 1993), $L_{X} / L_{R} \approx 10^{15.5}[\mathrm{~Hz}]^{2}$ with some scatter, and this correlation appears to mirror the behavior of individual solar flares (Benz and Güdel 1994). This flux-flux relation applies to quite different classes of active stars such as RS CVn binaries (Drake et al. 1989, 1992; Dempsey et al. 1993a, 1997; Fox et al. 1994) and active M dwarfs (Güdel et al. 1993). It is not entirely clear what the underlying cause is, but the most straightforward interpretation is in terms of chromospheric evaporation of frequent, unresolved flares that produce the observed radio emission and at the same time heat the plasma to coronal temperatures.

\section{Thermal structure of stellar coronae}

\subsection{Thermal coronal components}

The large range of temperatures measured in stellar coronae has been a challenge for theoretical interpretation from the early days of coronal research. Whereas much of the solar coronal plasma can be well described by a component of a few million degrees, early investigations of RS CVn binaries with low-resolution detectors already recognized that active stellar coronae cannot be (near-)isothermal but require a parameterization in terms of at least two largely different temperature components, one around 4-8 MK and the other around $20-100 \mathrm{MK}$ (Swank et al. 1981, also Holt et al. 1979, White et al. 1980, and Agrawal et al. 1981 for individual cases). First estimates based on static loop models showed that a simple active corona requires either very high pressures (of order 100 dynes $\mathrm{cm}^{-2}$ ), implying very compact sources with small filling factors, or extremely extended magnetic loops, with a possibility to connect to the binary companion (Swank et al. 1981). The solar analogy had thus immediately reached its limitation for a proper interpretation of stellar data. This theme and its variations have remained of fundamental interest to the stellar X-ray community ever since.

Although even low-resolution devices provide meaningful temperature measurements, there has been a long-standing debate on the interpretation of " $1-T$ " or " $2-T$ " models. Historically, the opinions were split; some argued that the individual temperature components represent separate plasma features (Schrijver et al. 1984; Mewe and Schrijver 1986; Singh et al. 1987; Pallavicini et al. 1988; Lemen et al. 1989; Pasquini et al. 1989; Schrijver et al. 1989b; Dempsey et al. 1993b; Singh et al. 1995, 1996a,c; Rodonò et al. 1999); others suggested that they parameterize a continuous distribution of EM in temperature and thus represent a continuum of source types (Majer et al. 1986; Schmitt et al. 1987, 1990a; Schmitt 1997; Drake et al. 1995b, 2001). As we have been learning from high-resolution spectroscopy, the correct solution may be a diplomatic one. There is little doubt (also from the solar analogy and simple physical models) that coronae display truly continuous EM distributions, but there are a number of superimposed features that may trace back to individual physical coronal structures. The differential emission measure distribution (DEM) thus became an interesting diagnostic

\footnotetext{
2 Note that the radio luminosity $L_{R}$ is conventionally derived from a flux density measured at a fixed frequency such as $4.9 \mathrm{GHz}$ or $8.4 \mathrm{GHz}$, per unit frequency interval.
} 
tool for coronal structure and heating (see, for example, Dupree et al. 1993; Brickhouse et al. 1995; Kaastra et al. 1996; Güdel et al. 1997a; Favata et al. 1997c; Griffiths and Jordan 1998, to mention a few).

The temperatures determined from low-resolution spectral devices may in fact also be driven by detector characteristics, in particular the accessible energy range as well as the spectral behavior of the detector's effective area (Majer et al. 1986; Schmitt et al. 1987; Pasquini et al. 1989; Schmitt et al. 1990a; Favata et al. 1997c). Overall, there is little doubt that the gross temperature determinations of low-resolution devices are correct, but a comprehensive description of the emission measure distribution requires high-resolution spectra that allow for more degrees of freedom and thus independent parameters, although the accuracy of the spectral inversion remains limited on principal mathematical grounds (see Sect. 9.3). I will in the following focus on more recent results that are based on reconstructions of full DEMs mainly from high-resolution devices, first reviewing some general data and basic definitions.

\section{High-resolution X-ray spectroscopy}

With the advent of Chandra and XMM-Newton, high-resolution X-ray spectroscopy has opened a new window to stellar coronal research. The Chandra High-Energy Transmission Grating Spectrometer (HETGS), the Low-Energy Transmission Grating Spectrometer (LETGS) as well as the two XMM-Newton Reflection Grating Spectrometers (RGS) cover a large range of spectral lines that can be separated and analyzed in detail. The spectra contain the features required for deriving emission measure distributions, abundances, densities, and opacities as discussed throughout this paper. Here, I give only a brief description of sample spectra and some distinguishing properties that are directly related to the thermal structure.

Figures 6 and 7 show examples of spectra obtained by XMM-Newton and Chandra, respectively. The stars cover the entire range of stellar activity: HR 1099 representing a very active RS CVn system, Capella an intermediately active binary, and Procyon an inactive F dwarf. The spectrum of HR 1099 reveals a considerable amount of continuum and comparatively weak lines, which is a consequence of the very hot plasma in this corona ( $T \approx 5-30 \mathrm{MK}$ ). Note also the unusually strong Ne IX/Fe XVII and Ne X/Fe XVII flux ratios if compared to the other stellar spectra. These anomalous ratios are in fact due to an abundance anomaly discussed in Sect. 16.3. The spectrum of Capella is dominated by Fe XVII and Fe XVIII lines which are preferentially formed in this corona's plasma at $T \approx 6 \mathrm{MK}$. Procyon, in contrast, shows essentially no continuum and only very weak lines of Fe. Its spectrum is dominated by the $\mathrm{H}$ - and He-like transitions of $\mathrm{C}, \mathrm{N}$, and $\mathrm{O}$ formed around 1-4 MK. The flux ratios between $\mathrm{H}$ - to He-like transitions are also convenient temperature indicators: The O VIII $\lambda 18.97 / O$ vII $\lambda 21.6$ flux ratio, for example, is very large for HR 1099 but drops below unity for Procyon. 


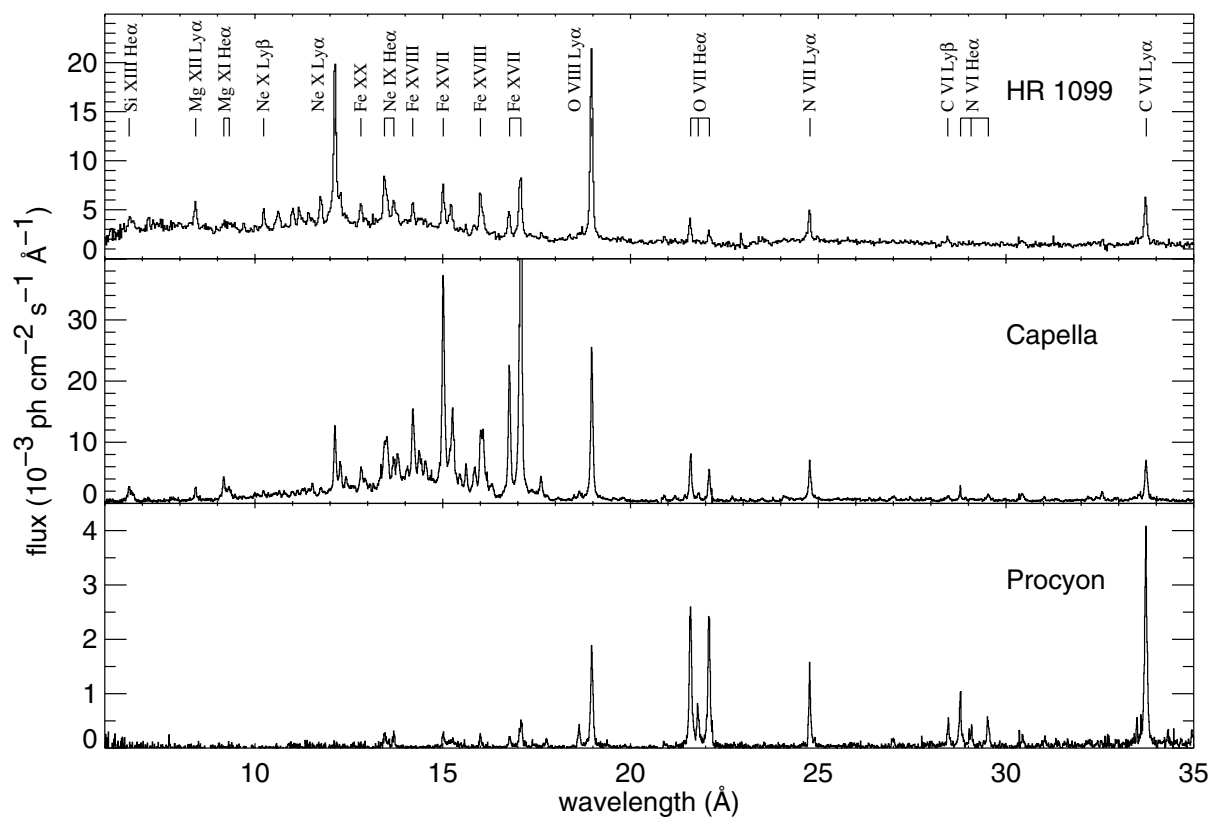

Fig. 6. Three high-resolution X-ray spectra of stars with largely differing activity levels: HR 1099, Capella, and Procyon. Data from XMM-Newton RGS

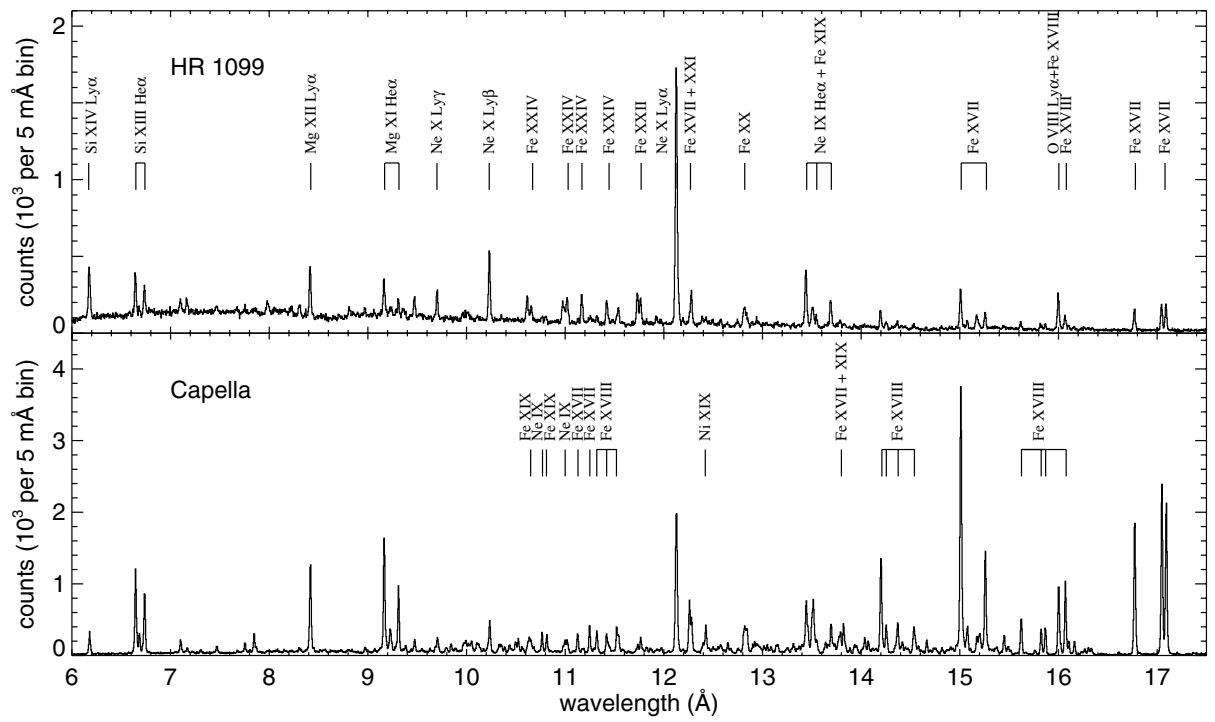

Fig. 7. Extracts of two high-resolution X-ray spectra from HR 1099 and Capella, showing the region of the Fe L-shell transitions. Strong lines in the Capella spectrum without identification labels correspond to those labeled in the HR 1099 spectrum (data from Chandra HETGS, courtesy of N. Brickhouse) 


\section{The differential emission measure distribution}

\subsection{Theory}

The flux $\phi_{j}$ observed in a line from a given atomic transition can be written as

$$
\phi_{j}=\frac{1}{4 \pi d^{2}} \int A G_{j}(T) \frac{n_{e} n_{H} d V}{d \ln T} d \ln T
$$

where $d$ denotes the distance, and $G_{j}(T)$ is the "line cooling function" (luminosity per unit EM) that contains the atomic physics of the transition as well as the ionization fraction for the ionization stage in question, and $A$ is the abundance of the element with respect to some basic tabulation used for $G_{j}$. For a fully ionized plasma with cosmic abundances, the hydrogen density $n_{H} \approx 0.85 n_{e}$. The expression

$$
Q(T)=\frac{n_{e} n_{H} d V}{d \ln T}
$$

defines the differential emission measure distribution (DEM). I will use this definition throughout but note that some authors define $Q^{\prime}(T)=n_{e} n_{H} d V / d T$ which is smaller by one power of $T$. For a plane-parallel atmosphere with surface area $S,(8)$ implies

$$
Q(T)=n_{e} n_{H} S H(T), \quad H(T)=\left|\frac{1}{T} \frac{d T}{d s}\right|^{-1}
$$

where $H$ is the temperature scale height.

\subsection{Interpretation}

Equations (7) and (8) introduce the DEM as the basic interface between the stellar X-ray observation and the model interpretation of the thermal source. It contains information on the plasma temperature and the density-weighted plasma mass that emits X-rays at any given temperature. Although a DEM is often a highly degenerate description of a complex real corona, it provides important constraints on heating theories and on the range of coronal structures that it may describe. Solar DEMs can, similarly to the stellar cases, often be approximated by two power laws $Q(T) \propto T^{s}$, one on each side of its peak. Raymond and Doyle (1981) reported low-temperature power-law slopes of $s=0.9$ for the coronal hole network, $s=2.1$ for the quiet Sun, and $s=3.1$ for flares. The Sun has given considerable guidance in physically interpreting the observed stellar DEMs, as the following subsections summarize.

\subsubsection{The DEM of a static loop}

The DEM of a hydrostatic, constant-pressure loop was discussed by Rosner et al. (1978) (= RTV), Vesecky et al. (1979) (= VAU), and Antiochos and Noci (1986). Under the conditions of negligible gravity, i.e., constant pressure in the entire loop, and negligible thermal conduction at the footpoints,

$$
Q(T) \propto p T^{3 / 4-\gamma / 2+\alpha} \frac{1}{\left(1-\left[T / T_{\mathrm{a}}\right]^{2-\gamma+\beta}\right)^{1 / 2}}
$$



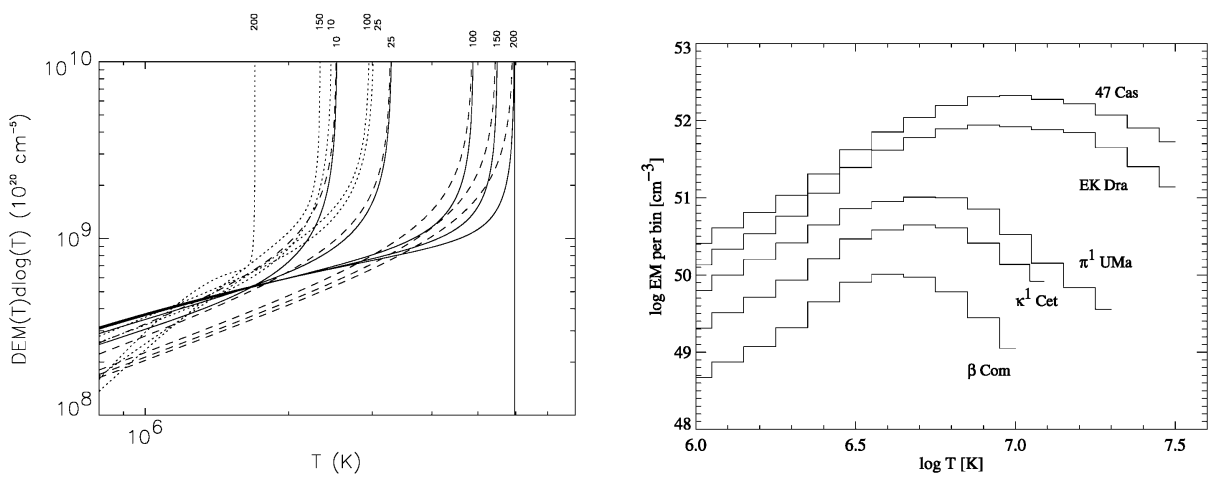

Fig. 8. Left: Calculated differential emission measures of individual static loops. The solid curves refer to uniform heating along the loop and some fixed footpoint heating flux, for different loop halflengths labeled above the figure panel in megameters. The dashed curves illustrate the analytical solutions presented by Rosner et al. (1978) for uniform heating. The dotted lines show solutions assuming a heating scale height of $2 \times 10^{9} \mathrm{~cm}$ (figure courtesy of K. Schrijver, after Schrijver and Aschwanden 2002). Right: Examples of discrete stellar emission measure distributions derived from spectra of solar analogs. The slopes below $\log T \approx 6.5$ are only approximate (figure courtesy of A. Telleschi)

(Bray et al. 1991) where $T_{\mathrm{a}}$ is the loop apex temperature, and $\alpha$ and $\beta$ are power-law indices of, respectively, the loop cross section area $S$ and the heating power $q$ as a function of $T: S(T)=S_{0} T^{\alpha}, q(T)=q_{0} T^{\beta}$, and $\gamma$ is the exponent in the cooling function over the relevant temperature range: $\Lambda(T) \propto T^{\gamma}$. If $T$ is not close to $T_{\mathrm{a}}$ and the loops have constant cross section $(\alpha=0)$, we have $Q(T) \propto T^{3 / 4-\gamma / 2}$, i.e., under typical coronal conditions for non-flaring loops $(T<10 \mathrm{MK}, \gamma \approx-0.5)$, the DEM slope is near unity (Antiochos and Noci 1986). If strong thermal conduction is included at the footpoints, then the slope changes to $+3 / 2$ if not too close to $T_{\mathrm{a}}$ (van den Oord et al. 1997), but note that the exact slope again depends on $\gamma$, i.e., the run of the cooling function over the temperature range of interest. The single-loop DEM sharply increases at $T \approx T_{\text {a }}$ (Fig. 8).

Such models may already resemble some stellar DEMs (Ayres et al. 1998), and they are close to observed solar full-disk DEMs that indicate $Q \propto T^{3 / 2}$ (Jordan 1980; Laming et al. 1995; Peres et al. 2001). However, the DEMs of many active stars are much steeper (see below; Fig. 8). Loop expansion $(\alpha>0)$ obviously steepens the DEM. Increased heating at the loop footpoints (instead of uniform heating) makes the $T$ range narrower and will also increase the slope of the DEM (Bray et al. 1991; Argiroffi et al. 2003). Further, if the heating is non-uniform, as for example in loops that are predominantly heated near the footpoints, the DEM becomes steeper as well (see numerical calculations of various loop examples by Schrijver and Aschwanden 2002 and Aschwanden and Schrijver 2002). Examples are illustrated in Fig. 8 together with discrete emission measure distributions derived from stellar spectra. Comprehensive numerical hydrostatic energybalance loop models undergoing steady apex heating have been computed by Griffiths (1999), with applications to observed DEMs, and by the Palermo group (see Sect. 11.2).

If the loops are uniformly distributed in $T_{\mathrm{a}}$, and one assumes a heating rate proportional to the square of the magnetic field strength, $B^{2}$, then $Q$ is dominated by the hottest 
loops because these produce more EM at any given $T$ than cooler loops (Antiochos and Noci 1986). In the more general case, the descending, high- $T$ slope is related to the statistical distribution of the loops in $T_{\mathrm{a}}$; a sharp decrease of the DEM indicates that only few loops are present with a temperature exceeding the temperature of the DEM peak (Peres et al. 2001).

Lemen et al. (1989) found that EM is concentrated at temperatures where the cooling function $\Lambda(T)$ has a positive slope or is flat; these are regions insensitive to heating fluctuations. This idea was further discussed by Gehrels and Williams (1993) who found that most 2- $T$ fits of low-resolution RS CVn spectra show plasma in two regions of relative stability, namely at 5-8 $\mathrm{MK}$ and above $25 \mathrm{MK}$.

\subsubsection{The DEM of flaring structures}

Antiochos (1980) (see also references therein) discussed DEMs of flaring loops that cool by i) static conduction (without flows), ii) evaporative conduction (including flows), and iii) radiation. The inferred DEMs scale, in the above order, like

$$
Q_{\text {cond }} \propto T^{1.5}, \quad Q_{\text {evap }} \propto T^{0.5}, \quad Q_{\text {rad }} \propto T^{-\gamma+1} .
$$

Since $\gamma \approx 0 \pm 0.5$ in the range typically of interest for stellar flares ( $5-50 \mathrm{MK})$, all above DEMs are relatively flat (slope $1 \pm 0.5$ ). If multiple loops with equal slope but different peak $T$ contribute, then the slope up to the first DEM peak can only become smaller. Non-constant loop cross sections have a very limited influence on the DEM slopes.

Stellar flare observations are often not of sufficient quality to derive temperature and EM characteristics for many different time bins. An interesting diagnostic was presented by Mewe et al. (1997) who calculated the time-integrated (average) DEM of a flare that decays quasi-statically. They find

$$
Q \propto T^{19 / 8}
$$

up to a maximum $T$ that is equal to the temperature at the start of the decay phase.

Sturrock et al. (1990) considered episodic flare heating. In essence, they showed how loop cooling together with the rate of energy injection as a function of $T$ may form the observed solar "quiescent" DEM, i.e., the latter would be related to the shape of the cooling function $\Lambda(T)$ : the negative slope of $\Lambda$ between $10^{5} \mathrm{~K}$ and a few times $10^{6} \mathrm{~K}$ results in an increasing $Q(T)$. Systems of this kind were computed semi-analytically by Cargill (1994), using analytic approximations for conductive and radiative decay phases of the flares. Here, the DEM is defined not by the internal loop structure but by the time evolution of a flaring plasma (assumed to be isothermal). Cargill argued that for radiative cooling, the (statistical) contribution of a flaring loop to the DEM is, to zeroth order, inversely proportional to the radiative decay time, which implies

$$
Q(T) \propto T^{-\gamma+1}
$$

up to a maximum $T_{m}$, and a factor of $T^{1 / 2}$ less if subsonic draining of the cooling loop is allowed. Simulations with a uniform distribution of small flares within a limited energy range agree with these rough predictions, indicating a time-averaged DEM that is relatively flat below $10^{6} \mathrm{~K}$ but steep $\left(Q[T] \propto T^{4}\right)$ up to a few $\mathrm{MK}$, a range in which 
the cooling function drops rapidly. Güdel (1997) used a semi-analytical hydrodynamic approach formulated by Kopp and Poletto (1993) to compute the time-averaged DEM for typical stellar conditions up to several $10^{7} \mathrm{~K}$ for a power-law distribution of the flare energies (see also Sect. 13.6), finding two DEM peaks that are dominated by the large number of small, cool flares ("microflares" producing the cooler plasma) and by the much less frequent energetic, hot flares, respectively.

Let us next assume - in analogy to solar flares - that the occurrence rate of flares is distributed in energy as a power law with an index $\alpha\left(d N / d E \propto E^{-\alpha}\right)$ and that the peak emission measure $\mathrm{EM}_{p}$ of a flare is a power-law function of its peak temperature $T_{p}$ at least over a limited range of temperatures: $\mathrm{EM}_{p} \propto T_{p}^{b}$ as found by Feldman et al. (1995) (see also Sect. 12.12 for a larger flare sample). Then, an analytic expression can be derived for the time-averaged DEM of such a flare ensemble, i.e., a "flare-heated corona", revealing a power law on each side of the DEM peak (Güdel et al. 2003a):

$$
Q(T) \propto \begin{cases}T^{2 / \zeta} & \text { for } T<T_{m} \\ T^{-(\alpha-2)(b+\gamma)-\gamma} & \text { for } T>T_{m}\end{cases}
$$

where we have assumed the same luminosity decay-time scale for all flares. Here, $T_{m}$ (a free parameter) is the temperature of the DEM peak, and $b \approx 4.3 \pm 0.35$ (Sect. 12.12) in the temperature range of interest for active-stellar conditions. The parameter $\zeta \equiv \tau_{n} / \tau_{T}$, $\tau_{n}$ and $\tau_{T}$ being the e-folding decay times of density and temperature, respectively, is found to vary between $\zeta=0.5$ (strong heating during the decay) and $\zeta=2$ (no heating, see Reale et al. 1997, Sect. 12.6). This model produces DEM slopes below $T_{m}$ that are steeper than unity and range up to a maximum of four.

\subsection{Reconstruction methods and limitations}

Deriving DEMs or their discretized, binned equivalents, the "emission measure distributions" (EMD) in $\log T$ from X-ray spectra has been one of the central issues in observational stellar X-ray astronomy. As implied by (7), it is also of considerable importance in the context of determining the coronal composition (see Sect. 16). Although a full description of the methodology of spectral inversion is beyond the scope of this review, I will briefly outline the available strategies as well as the current debate on optimizing results. This may serve as an introduction and guide to the more technical literature.

If a spectrum of an isothermal plasma component with unit EM is written in vector form as $\mathbf{f}(\lambda, T)$, then the observed spectrum is the weighted sum

$$
\mathbf{g}(\lambda)=\int \mathbf{f}(\lambda, T) Q(T) d \ln T \equiv \mathbf{F} \cdot \mathbf{Q} .
$$

In discretized form for bins $\Delta \log T, \mathbf{F}$ is a rectangular matrix (in $\lambda$ and $T$ ). Equation (15) constitutes a Fredholm equation of the first kind for $\mathbf{Q}$. Its inversion aiming at solving for $\mathrm{Q}$ is an ill-conditioned problem with no unique solution unless one imposes additional constraints such as positivity, smoothness, or functional form, most of which may not be physically founded. A formal treatment is given in Craig and Brown (1976). The problem is particularly serious due to several sources of unknown and systematic uncertainties, 
such as inaccurate atomic physics parameters in the spectral models, uncertainties in the instrument calibration and imprecise flux determinations, line blends (see detailed discussion in van den Oord et al. 1997 and Kashyap and Drake 1998 and references therein) and, in particular, unknown element abundances. The latter need to be determined from the same spectra. They are usually assumed to be constant across the complete DEM although this hypothesis is not supported by solar investigations (Laming et al. 1995; Jordan et al. 1998). The following constrained inversion techniques have turned out to be convenient: ${ }^{3}$

1. Integral inversion with regularization. A matrix inversion of (15) is used with the additional constraint that the second derivative of the solution $Q(\log T)$ is as smooth as statistically allowed by the data. Oscillations in the data that are due to data noise are thus damped out. This method is appropriate for smooth DEMs, but tends to produce artificial wings in sharply peaked DEMs (Mewe et al. 1995; Schrijver et al. 1995; Cully et al. 1997). A variant using singular value decomposition for a series of measured line fluxes was discussed by Schmitt et al. (1996b) .

2. Multi-temperature component fits. This approach uses a set of elementary DEM building blocks such as Gaussian DEMs centered at various $T$ but is otherwise similar to the traditional multi-component fits applied to low-resolution data (examples were given by Kaastra et al. 1996 and Güdel et al. 1997b).

3. Clean algorithm. This is both a specific iteration scheme and a special case of (2) that uses delta functions as building blocks. The observed spectrum (or part of it) is correlated with predictions from isothermal models. The model spectrum with the highest correlation coefficient indicates the likely dominant $T$ component. A fraction of this spectral component is subtracted from the observation, and the corresponding model EM is saved. This process is iterated until the residual spectrum contains only noise. The summed model EM tends to produce sharp features while positivity is ensured (Kaastra et al. 1996).

4. Polynomial DEMs. The DEM is approximated by the sum of Chebychev polynomials $P_{k}$. For better convergence, the logarithms of the EM and of $T$ are used: $\log [Q(T) d \log (T)]=\sum_{k=0}^{N-1} a_{k} P_{k}(\log T)$ which ensures positivity. The degree $N$ of the polynomial fit can be adjusted to account for broad and narrow features (Lemen et al. 1989; Kaastra et al. 1996; Schmitt and Ness 2004; Audard et al. 2004).

5. Power-law shaped DEMs of the form $Q(T) \propto\left(T / T_{\max }\right)^{\alpha}$ up to a cutoff temperature $T_{\max }$ are motivated by the approximate DEM shape of a single magnetic loop (Pasquini et al. 1989; Schmitt et al. 1990a).

3 I henceforth avoid expressions such as "global" or "line-based methods" that have often been used in various, ill-defined contexts. Spectral inversion methods should be distinguished by i) the range and type of the data to be fitted, ii) the parameters to be determined (model assumptions), iii) the iteration scheme for the fit (if an iterative technique is applied), iv) the convergence criteria, v) the constraints imposed on the solution (e.g., functional form of DEM, smoothness, positivity, etc), and vi) the atomic database used for the interpretation. Several methods described in the literature vary in some or all of the above characteristics. Most of the methods described here are not inherently tailored to a specific spectral resolving power. What does require attention are the possible biases that the selected iteration scheme and the constraints imposed on the solution may introduce, in particular because the underlying atomic physics tabulations are often inaccurate or incomplete ("missing lines" in the codes). 
The ranges and types of data may vary depending on the data in use. Low-resolution spectra are commonly inverted as a whole because individual features cannot be isolated. If a high-resolution spectrum is available, then inversion methods have been applied either to the entire spectrum, to selected features (i.e., mostly bright lines), or to a sample of extracted line fluxes.

As for iteration schemes, standard optimization/minimization techniques are available. Various methods have been developed for fits to samples of line fluxes (e.g., Lemen et al. 1989; Huenemoerder et al. 2001, 2003; Osten et al. 2003; Sanz-Forcada et al. 2003; Telleschi et al. 2004), with similar principles:

1. The DEM shape is iteratively derived from line fluxes of one element only, typically $\mathrm{Fe}$ (XVII-XXVI in X-rays, covering $T$ up to $\approx 100 \mathrm{MK}$ ), e.g., by making use of one of the above inversion schemes tailored to a sample of line fluxes. Alternatively, one can use $T$-sensitive but abundance-independent flux ratios between He-like and H-like transitions of various elements to construct the DEM piece-wise across a temperature range of $\approx 1-15 \mathrm{MK}$ (Schmitt and Ness 2004).

2. The Fe abundance (and thus the DEM normalization) is found by requiring that the continuum (formed mainly by $\mathrm{H}$ and $\mathrm{He}$ ) agrees with the observations.

3. The abundances of other elements are found by comparing their DEM-predicted line fluxes (e.g., assuming solar abundances), with the observations.

The advantage of such schemes is that they treat the DEM inversion and the abundance determination sequentially and independently. Huenemoerder et al. (2001) and Huenemoerder et al. (2003) used an iteration scheme that fits DEM and abundances simultaneously based on a list of line fluxes plus a continuum. Kashyap and Drake (1998) further introduced an iteration scheme based on Markov-chain Monte Carlo methods for a list of line fluxes. This approach was applied to stellar data by Drake et al. (2001). Genetic algorithms have also, albeit rarely, been used as iteration schemes (Kaastra et al. 1996 for low-resolution spectra).

There has been a lively debate in the stellar community on the "preferred" spectral inversion approach. Some of the pros and cons for various strategies are: Methods based on full, tabulated spectral models or on a large number of individual line fluxes may be compromised by inclusion of transitions with poor atomic data such as emissivities or wavelengths. On the other hand, a large line sample may smooth out the effect of such uncertainties. Consideration of all tabulated lines further leads to a treatment of line blends that is self-consistent within the limits given by the atomic physics uncertainties. A most serious problem arises from weak lines that are not tabulated in the spectral codes while they contribute to the spectrum in two ways: either in the form of excess flux that may be misinterpreted as a continuum, thus modifying the DEM; or in the form of unrecognized line blends, thus modifying individual line fluxes and the pedestal flux on which individual lines are superimposed. A careful selection of spectral regions and lines for the inversion is thus required (see discussion and examples in Lepson et al. 2002 for the EUV range).

If DEMs and abundances are iterated simultaneously, numerical cross-talk between abundance and DEM calculation may be problematic, in particular if multiple solutions exist. Nevertheless, each ensemble of line flux ratios of one element determines the same DEM and thus simultaneously enforces agreement. If a list of selected line fluxes 
is used, e.g., for one element at a time, DEM-abundance cross talk can be avoided, and the influence of the atomic physics uncertainties can be traced throughout the reconstruction process. But there may be a strong dependence of the reconstruction on the atomic physics uncertainties and the flux measurements of a few lines. The lack of a priori knowledge on line blends affecting the extracted line fluxes will introduce systematic uncertainties as well. This can, however, be improved if tabulated potential line blends are iteratively included.

The presence of systematic uncertainties also requires a careful and conservative choice of convergence criteria or smoothness parameters to avoid introduction of spurious features in the DEM. The result is a range of solutions that acceptably describe the data based on a goodness-of-fit criterion, in so far as the data can be considered to be represented by the spectral database in use. Within this allowed range, "correctness" cannot be judged on by purely statistical arguments. The spectral inversion is non-unique because the mathematical problem is ill-posed - the atomic data deficiencies cannot be overcome by statistical methodology but require external information.

Direct comparisons of various methods, applied to the same data, are needed. Mewe et al. (2001) presented an EMD for Capella based on selected Fe lines that compares very favorably with their EMD derived from a multi- $T$ approach for the complete spectra, and these results also seem to agree satisfactorily with previously published EMDs from various methods and various data sets. EMDs of the active HR 1099 found from spectra of XMM-Newton RGS (Audard et al. 2001a) and from Chandra HETGS (Drake et al. 2001) agree in their principal features, notwithstanding the very different reconstruction methods applied and some discrepancies in the abundance determinations. Telleschi et al. (2004) determined EMDs and abundances of a series of solar analogs at different activity levels from polynomial-DEM fits to selected spectral regions and from an iterative reconstruction by use of extracted line-flux lists. The resulting EMDs and the derived abundances of various elements are in good agreement. Schmitt and Ness (2004) compared two approaches within their polynomial DEM reconstruction method, again concluding that the major discrepancies result from the uncertainties in the atomic physics rather than from the reconstruction approach, in particular when results from EUV lines are compared with those from X-ray lines. A somewhat different conclusion was reported by Sanz-Forcada et al. (2003) from an iterative analysis of Fe-line fluxes of AB Dor; nevertheless, their abundance distribution is in fact quite similar to results reported by Güdel et al. (2001b) who fitted a complete spectrum.

The evidence hitherto reported clearly locates the major obstacle not in the inversion method but in the incompleteness of, and the inaccuracies in, the atomic physics tabulations. Brickhouse et al. (1995) gave a critical assessment of the current status of Fe line emissivities and their discrepancies in the EUV range, together with an analysis of solar and stellar spectra. The effects of missing atomic transitions in the spectral codes were demonstrated by Brickhouse et al. (2000) who particularly discussed the case of Fe transitions from high $n$ quantum numbers, i.e., of transitions that have only recently been considered. 


\subsection{Observational results}

If the caveats and the principal mathematical limitations of the present state of the art in the derivation of EMDs discussed above are properly taken into account, then the physical implications of some of the more secure results offer access to the underlying coronal physics. Most EMDs have generally been found to be singly or doubly peaked (Mewe et al. 1995, 1996, 1997; Drake et al. 1995b, 1997; Rucinski et al. 1995; Schrijver et al. 1995; Kaastra et al. 1996; Schmitt et al. 1996b; Güdel et al. 1997a,b) and confined on either side approximately by power laws (e.g., Mathioudakis and Mullan 1999; Güdel et al. 2003a). Examples are shown in Figs. 8 and 9, where stellar and solar EMDs are

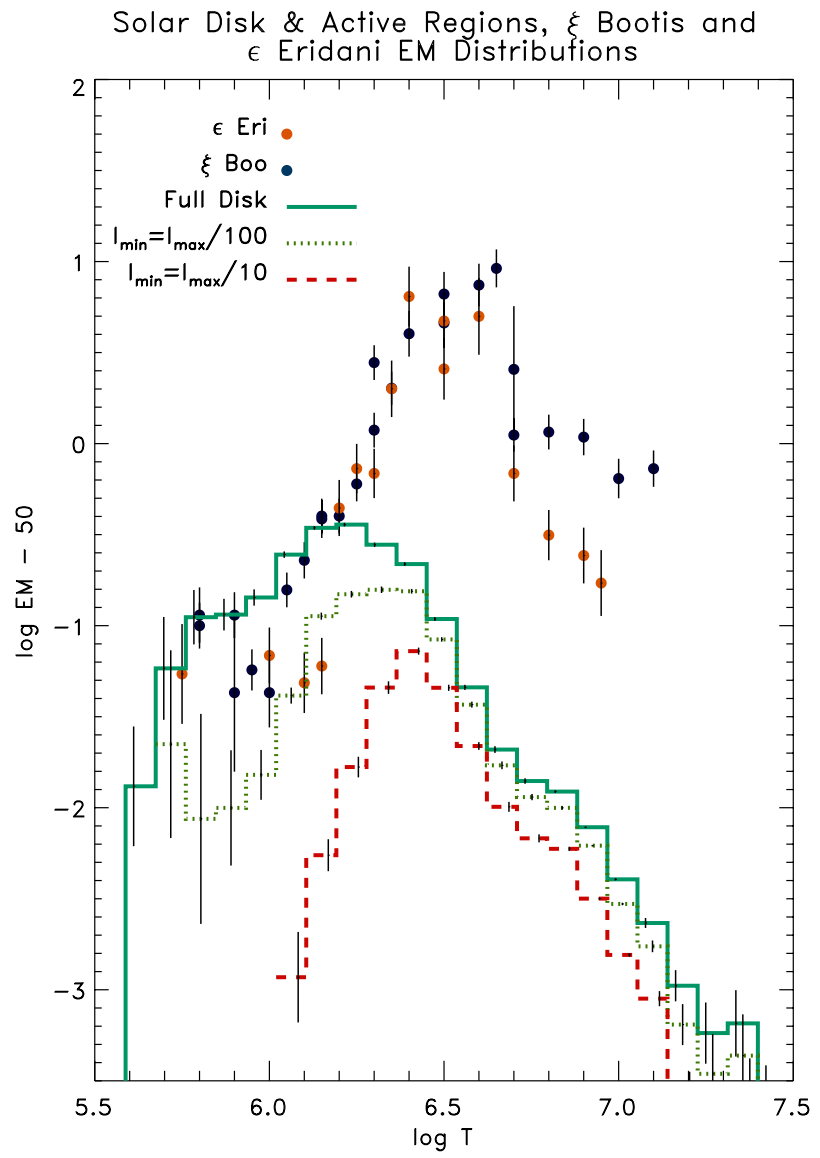

Fig. 9. Emission measure distributions of two intermediately active stars and the Sun. The axes are logarithmic, with the EM given in units of $10^{50} \mathrm{~cm}^{-3}$, and the temperature in $\mathrm{K}$. The blue and red circles refer to EMDs for $\xi$ Boo and $\epsilon$ Eri, respectively. The histograms refer to full-disk solar EMDs derived from Yohkoh images at solar maximum, including also two versions for different lower cutoffs for the intensities in Yohkoh images. Note the similar low- $T$ shapes but the additional high- $T$ contributions in the stars that reveal EMDs similar to bright solar active regions (figure courtesy of J. Drake, after Drake et al. 2000) 
compared. These power laws open up interesting ways of interpretation as discussed in Sect. 9.2.1 and 9.2.2.

It is notable that the complete EMD shifts to higher temperatures with increasing stellar activity (see also Sect. 9.5), often leaving very little EM at modest temperatures and correspondingly weak spectral lines of C, N, and O (Kaastra et al. 1996). For example, Haisch et al. (1994) found only highly-ionized spectral lines in the EUV spectrum of the intermediately active solar analog $\chi^{1}$ Ori (Fe XVI and higher) whereas the EUV spectrum of Procyon and $\alpha$ Cen is dominated by lower ionization stages, corresponding to a DEM peaking at 1-2 MK, similar to the full-disk, non-flaring solar DEM (Mewe et al. 1995; Drake et al. 1995b, 1997).

Double peaks are often found for active stars. They then reveal a minimum in the range of 10-20 MK (Lemen et al. 1989; Mewe et al. 1996; Güdel et al. 1997a; Huenemoerder et al. 2003). Filling in this trough does not seem to lead to consistent solutions (e.g., Lemen et al. 1989; Sanz-Forcada et al. 2001), although bias could be introduced by inaccurate atomic physics. On the other hand, the two peaks may have a physical foundation. Continual flaring has been proposed (Güdel 1997), or separate families of magnetic loops dominated by two different temperature regimes (Sciortino et al. 1999). In less active stars, the hotter peak disappears, leaving a marked single EMD maximum just below 10 MK (Dupree et al. 1993; Brickhouse and Dupree 1998; Sanz-Forcada et al. 2001, 2002).

EMDs are often steeper on the low- $T$ side than single, constant-cross section loop models (e.g., the static loop models by Rosner et al. 1978) predict, and this is particularly true for the more active stars (Pasquini et al. 1989; Schmitt et al. 1990a; Dempsey et al. 1993b; Laming et al. 1996; Laming and Drake 1999; Drake et al. 2000; Sanz-Forcada et al. 2002; Scelsi et al. 2004; Telleschi et al. 2004). For example, the cooler branches of the stellar EMDs in Fig. 9 follow approximately $Q \propto T^{3}$. One remedy may be loops with an expanding cross section from the base to the apex, as computed by Vesecky et al. (1979). In that case, there is comparatively more hot plasma, namely the plasma located around the loop apex, than cooler plasma. The EMD and the DEM would consequently steepen. Spectral fits using individual loops require, in specific cases, expansion factors $\Gamma$, i.e., the ratio between cross sectional areas at the apex and at the footpoints, of between 2 and 50. Still, this model may fail for some sources (Schrijver et al. 1989b; Ottmann 1993; Schrijver and Aschwanden 2002). Alternatively, the steep low- $T$ EMD slopes may be further evidence for continual flaring (Güdel et al. 2003a); (14) predicts slopes between 1 and 4, similar to what is often found in magnetically active stars (see Sect. 13.6).

An extremely steep (slope of 3-5) EMD has consistently been derived for Capella (Brickhouse et al. 1995; Audard et al. 2001b; Behar et al. 2001; Mewe et al. 2001; Argiroffi et al. 2003). Here, the EMD shows a sharp peak around 6 MK that dominates the overall spectrum. These EM results also agree well with previous analyses based on EUV spectroscopy (Brickhouse et al. 2000).

\subsection{Coronal temperature-activity relations}

Early low-resolution spectra from HEAO 1, Einstein, EXOSAT, and ROSAT implied the persistent presence of considerable amounts of plasma at unexpectedly high temperatures, $T>10$ MK (e.g., Walter et al. 1978b; Swank et al. 1981), particularly in extremely 
active stars. This is now borne out by EMDs of many active stars that show significant contributions up to 30-40 MK, if not higher.

One of the surprising findings from stellar X-ray surveys is a relatively tight correlation between the characteristic coronal temperature and the normalized coronal luminosity $L_{X} / L_{\mathrm{bol}}$ : Stars at higher activity levels support hotter coronae (Vaiana 1983; Schrijver et al. 1984; Stern et al. 1986; Schmitt et al. 1990a; Dempsey et al. 1993b; Maggio et al. 1994; Gagné et al. 1995a; Schmitt et al. 1995; Hünsch et al. 1996; Güdel et al. 1997a; Preibisch 1997a; Schmitt 1997; Singh et al. 1999). Instead of explicit temperature measurements, some authors used spectral hardness as a proxy for $T$, and the surface flux $F_{X}$ or the EM per unit area can be used instead of $L_{X} / L_{\mathrm{bol}}$, but the conclusions remain the same. The example of solar analogs is shown together with the Sun itself during its activity maximum and minimum in Fig. 10. Here,

$$
\begin{aligned}
L_{X} & \propto T^{4.5 \pm 0.3} \\
E M & \propto T^{5.4 \pm 0.6}
\end{aligned}
$$

where $L_{X}$ denotes the total X-ray luminosity, but EM and $T$ refer to the "hotter" component in standard 2- $T$ fits to ROSAT data. Such relations extend further into the pre-main sequence domain where exceedingly hot coronae with temperatures up to $\approx 100 \mathrm{MK}$ are found (Imanishi et al. 2001a).

The cause of this relation is not clear. Three classes of models might apply: Phenomenologically, as the activity on a star increases, the corona becomes progressively more dominated by hotter and denser features, for example active regions as opposed to quiet areas or coronal holes. Consequently, the average stellar X-ray spectrum indicates more hot plasma (Schrijver et al. 1984; Maggio et al. 1994; Güdel et al. 1997a; Preibisch 1997a; Orlando et al. 2000; Peres et al. 2000; see Sect. 11.4).

Increased magnetic activity also leads to more numerous interactions between adjacent magnetic field structures. The heating efficiency thus increases. In particular, we expect a higher rate of large flares. Such models naturally produce the temperatureactivity correlation: The increased flare rate produces higher X-ray luminosity because chromospheric evaporation produces more EM; at the same time, the plasma is heated to higher temperatures in larger flares (as we will discuss in Sect. 12.12). This mechanism was simulated and discussed by Güdel et al. (1997a), Güdel (1997), and Audard et al. (2000) (see also Sect. 13).

Jordan et al. (1987) and Jordan and Montesinos (1991) studied an EM-T relation based on arguments of a minimum energy loss configuration of the corona, assuming a fixed ratio between radiative losses and the coronal conductive loss. They suggested a relation including the stellar gravity $g$ of the form

$$
E M \propto T^{3} g
$$

which fits quite well to a sample of observations with $T$ taken from single- $T$ fits to stellar coronal data. The authors suggested that this relation holds because coronal heating directly relates to the production rate of magnetic fields, and the magnetic pressure is assumed to scale with the thermal coronal pressure. Equation (18) then follows directly. 


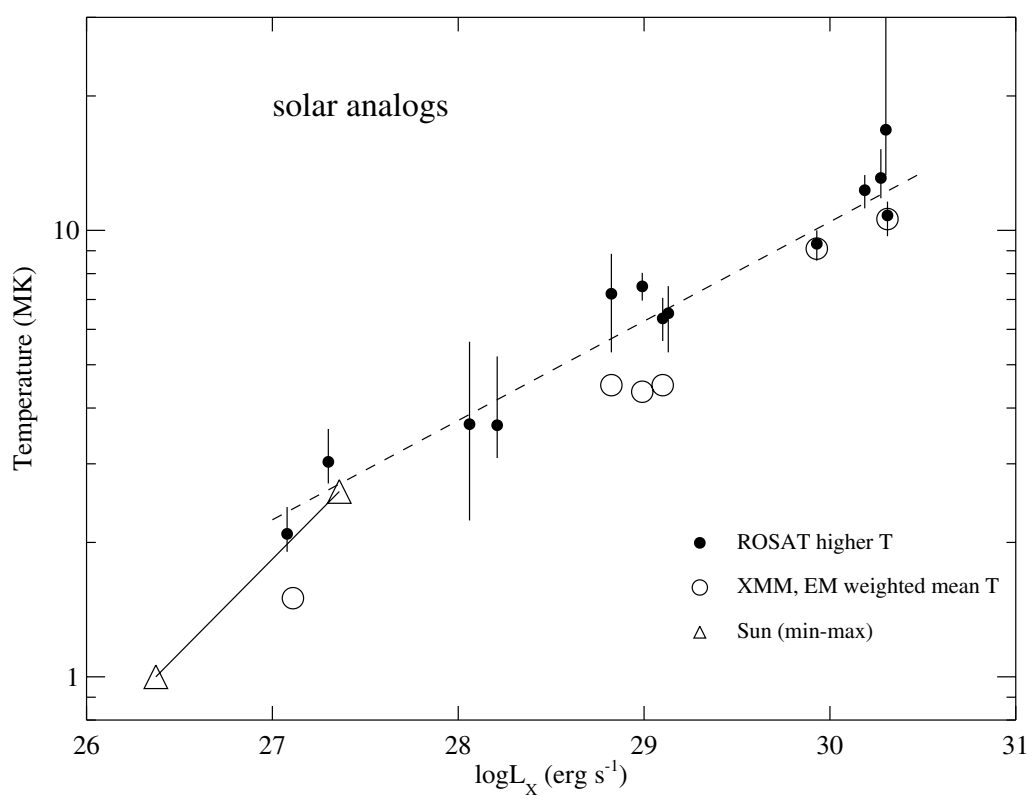

Fig. 10. Coronal temperature vs. X-ray luminosity for solar analogs. The filled circles are from the sample of Güdel et al. (1997a) and Güdel et al. (1998) and refer to the hotter component in 2-T fits to ROSAT data. The solar points (triangles), covering the range from sunspot minimum to maximum, were taken from Peres et al. (2000). The open circles refer to EM-weighted averages of $\log T$; these values were derived from full reconstructed EMDs (Telleschi et al. 2004) and, for $\alpha$ Cen (at $L_{X} \approx 10^{27} \mathrm{erg} \mathrm{s}^{-1}$ ), from multi- $T$ fits (Raassen et al. 2003a). In both of the latter cases, XMM-Newton data were used

\section{Electron densities in stellar coronae}

With the advent of high-resolution spectroscopy in the EUV range (by EUVE and Chandra) and in X-rays (by XMM-Newton and Chandra), spectroscopic tools have become available to measure electron densities $n_{e}$ in coronae. Coronal electron densities are important because they control radiative losses from the coronal plasma; observationally, they can in principle also be used in conjunction with EMs to derive approximate coronal source volumes. The spectroscopic derivation of coronal densities is subtle, however. Two principal methods are available.

\subsection{Densities from Fe line ratios}

The emissivities of many transitions of Fe ions in the EUV range are sensitive to densities in the range of interest to coronal research (Mewe et al. 1985, 1995; Schmitt et al. 1994a, 1996c; Mathioudakis and Mullan 1999). Brickhouse et al. (1995) have provided extensive tabulations of the relevant emissivities together with a critical review of their overall reliability. The different density dependencies of different lines of the same $\mathrm{Fe}$ ion then also make their line-flux ratios, which (apart from blends) are easy to measure, useful diagnostics for the electron density. 
A review of the literature (see also Bowyer et al. 2000) shows a rather unexpected segregation of coronal densities into two realms at different temperatures. The cool coronal plasma in inactive stars is typically found at low densities of order $10^{9} \mathrm{~cm}^{-3}$ $-10^{10} \mathrm{~cm}^{-3}$. In active stars, the cooler components may show elevated densities, but extreme values up to $>10^{13} \mathrm{~cm}^{-3}$ have been reported for the hotter plasma component. A few notable examples follow.

For the inactive $\alpha$ Cen, Mewe et al. (1995) derived $n_{e}=2 \times 10^{8} \mathrm{~cm}^{-3}-2 \times 10^{9} \mathrm{~cm}^{-3}$, in reasonable agreement with a measurement from EUVE (Drake et al. 1997) and with typical solar coronal densities (Landi and Landini 1998). For the similarly inactive Procyon, Schmitt et al. (1994a, 1996c) and Schrijver et al. (1995) found somewhat higher values of approximately $3 \times 10^{9} \mathrm{~cm}^{-3}-4 \times 10^{9} \mathrm{~cm}^{-3}$ from lines of Fe X-XIV, with an allowed range from $10^{9} \mathrm{~cm}^{-3}$ to $10^{10} \mathrm{~cm}^{-3}$.

This picture does not change much for intermediately active stars such as $\epsilon$ Eri $\left(n_{e} \approx\right.$ $3 \times 10^{9} \mathrm{~cm}^{-3}$, Laming et al. 1996; Schmitt et al. 1996b), or $\xi$ Boo A $\left(n_{e} \gtrsim 10^{10} \mathrm{~cm}^{-3}\right.$, Laming and Drake 1999). In general, the more active stars tend to show somewhat higher densities.

An appreciable change comes with higher activity levels when we consider the hotter plasma. Some extremely high densities have been reported from line ratios of highly ionized Fe, for example: Capella $\left(n_{e}=[0.04-1.5] \times 10^{13} \mathrm{~cm}^{-3}\right.$ from Fe XIX-XXII; Dupree et al. 1993; Schrijver et al. 1995), UX Ari $\left(n_{e}=[4.5 \pm 2] \times 10^{12} \mathrm{~cm}^{-3}\right.$ from Fe xxi; Güdel et al. 1999, also Sanz-Forcada et al. 2002), AU Mic $\left(n_{e}=[2-5] \times\right.$ $10^{12} \mathrm{~cm}^{-3}$ from Fe XXI-XXII; Schrijver et al. 1995), $\sigma$ Gem $\left(n_{e} \approx 10^{12} \mathrm{~cm}^{-3}\right.$ from Fe XXI-XXII; Schrijver et al. 1995, also Sanz-Forcada et al. 2002), $\xi$ UMa B $\left(n_{e}=\right.$ $5 \times 10^{12} \mathrm{~cm}^{-3}$ from Fe XXI-XXII; Schrijver et al. 1995), 44i Boo $\left(n_{e}>10^{13} \mathrm{~cm}^{-3}\right.$ from Fe XIX; Brickhouse and Dupree 1998), HR $1099\left(n_{e}=[1.6 \pm 0.4] \times 10^{12} \mathrm{~cm}^{-3}\right.$ from Fe XIX, XXI, XXII; Sanz-Forcada et al. 2002; somewhat higher values in Osten et al. 2004), II Peg $\left(n_{e}=[2.5-20] \times 10^{12} \mathrm{~cm}^{-3}\right.$ from Fe XXI and XxII; Sanz-Forcada et al. 2002), AB Dor $\left(n_{e}=[2-20] \times 10^{12} \mathrm{~cm}^{-3}\right.$ from Fexx-XXII; Sanz-Forcada et al. 2002), $\beta$ Cet $\left(n_{e}=[2.5-16] \times 10^{11} \mathrm{~cm}^{-3}\right.$ from Fexxi; Sanz-Forcada et al. 2002), and several short-period active binaries $\left(n_{e} \approx[3-10] \times 10^{12} \mathrm{~cm}^{-3}\right.$ from Fe XXI, Osten et al. 2002). However, most of these densities are only slightly above the lowdensity limits for the respective ratios, and upper limits have equally been reported (for $\sigma^{2} \mathrm{CrB}, n_{e} \lesssim 10^{12} \mathrm{~cm}^{-3}$ from Fe XxI, Osten et al. 2000, 2003; for HD 35850, $n_{e} \leq[4-50] \times 10^{11} \mathrm{~cm}^{-3}$ from Fe XxI, Mathioudakis and Mullan 1999; Gagné et al. 1999).

Mewe et al. (2001) used several line ratios of Fe XX, XXI, and XXII in Chandra data of Capella, reporting $n_{e} \lesssim(2-5) \times 10^{12} \mathrm{~cm}^{-3}$, in mild contradiction with $E U V E$ reports cited above. More stringent upper limits of $\log n_{e}=11.52$ were obtained for Algol from Fe Xxi EUV line ratios (Ness et al. 2002b). From a detailed consideration of Fe XXI line ratios in the Chandra HETG spectrum of Capella, Phillips et al. (2001) even concluded that the density measured by the most reliable Fe xxI line ratio, $f(\lambda 102.22) / f(\lambda 128.74)$, is compatible with the low-density limit of this diagnostic (i.e., $n_{e} \lesssim 10^{12} \mathrm{~cm}^{-3}$ ); in fact, these authors re-visited previous measurements of the same ratio and suggested that they all represent the low-density limit for Capella. Likewise, Ayres et al. (2001a) find contradictory results from different density indicators in the EUV spectrum of $\beta$ Cet, and suggest low densities. Finally, Ness et al. (2004) measured various Fe line ratios in a 
large sample of coronal stars. None of the stars showed high densities from all line ratios, and all values were again close to the low-density limit; moreover, a given line flux ratio appears to be identical for all considered stars, within the uncertainties. Because it is unlikely that all coronae reveal the same densities, a more natural assumption is that all measurements represent the low-density limit.

The observational situation is clearly unsatisfactory at the time of writing. It is worrisome that most measurements referring to the hotter plasma straddle the low-density limit of the respective ion but tend to be systematically different for ionization stages that have similar formation temperatures. At face value, it is perhaps little surprising that the densities do not come out even higher, but this circumstance makes the measurements extremely vulnerable to systematic but unrecognized inaccuracies in the atomic physics tabulations, and to unrecognized blends in some of the lines. Slight shifts then have a dramatic effect on the implied densities, as can be nicely seen in the analysis presented by Phillips et al. (2001). The resolution of these contradictions requires a careful reconsideration of atomic physics issues.

\subsection{Line ratios of He-like ions}

The He-like triplets of C v, N vI, O vII, Ne IX, Mg XI, and Si XIII provide another interesting density diagnostic for stellar coronae. Two examples are shown in Fig. 11 (right). The spectra show, in order of increasing wavelength, the resonance, the intercombination, and the forbidden line of the $\mathrm{O}$ VII triplet. The ratio between the fluxes in the forbidden line and the intercombination line is sensitive to density (Gabriel and Jordan 1969) for the following reason: if the electron collision rate is sufficiently high, ions in the upper
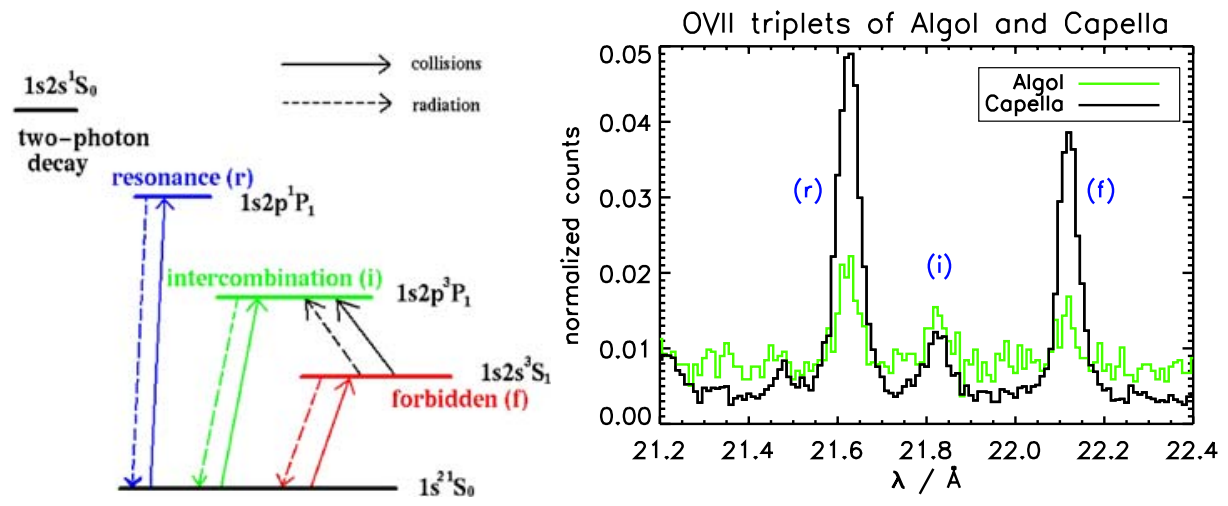

Fig. 11. Left: Term diagram for transitions in He-like triplets. The resonance, intercombination, and forbidden transitions are marked. The transition from ${ }^{3} S_{1}$ to ${ }^{3} P_{1}$ re-distributes electrons from the upper level of the forbidden transition to the upper level of the intercombination transition, thus making the $f / i$ line-flux ratio density sensitive. In the presence of a strong UV field, however, the same transition can be induced by radiation as well. Right: He-like triplet of O VII for Capella (black) and Algol (green). The resonance (r), intercombination (i), and forbidden (f) lines are marked. The $f / i$ flux ratio of Algol is suppressed probably due to the strong UV radiation field of the primary B star (data from Chandra; both figures courtesy of J.-U. Ness) 
level of the forbidden transition, $1 s 2 s^{3} S_{1}$, do not return to the ground level, $1 s^{2}{ }^{1} S_{0}$, instead the ions are collisionally excited to the upper levels of the intercombination transitions, $1 s 2 p^{3} P_{1,2}$, from where they decay radiatively to the ground state (see Fig. 11 for a term diagram). They thus enhance the flux in the intercombination line and weaken the flux in the forbidden line. The measured ratio $R=f / i$ of the forbidden to the intercombination line flux can be written as

$$
R=\frac{R_{0}}{1+n_{e} / N_{c}}=\frac{f}{i}
$$

where $R_{0}$ is the limiting flux ratio at low densities and $N_{c}$ is the critical density at which $R$ drops to $R_{0} / 2$. For $\mathrm{Cv}$ and $\mathrm{NvI}$, the photospheric radiation field needs to be considered as well because it enhances the ${ }^{3} S_{1}-{ }^{3} P_{1,2}$ transitions; the same applies to higher- $Z$ triplets if a hotter star illuminates the X-ray source (see Ness et al. 2001). The tabulated parameters $R_{0}$ and $N_{c}$ are slightly dependent on the electron temperature in the emitting source; this average temperature can conveniently be confined by the temperature-sensitive $G$ ratio of the same lines, $G=(i+f) / r$ (here, $r$ is the flux in the resonance line $\left.1 s^{2}{ }^{1} S_{0}-1 s 2 p^{1} P_{1}\right)$. A recent comprehensive tabulation is given in Porquet et al. (2001); Table 2 contains relevant parameters for the case of a plasma that is at the maximum formation temperature of the respective ion. A systematic problem with He-like triplets is that the critical density $N_{c}$ increases with the formation temperature of the ion, i.e., higher- $Z$ ions measure only high densities at high $T$, while the lower-density analysis based on $\mathrm{CV}, \mathrm{N}$ VI, O VII, and Ne IX is applicable only to cool plasma.

Stellar coronal He-like triplets have become popular with Chandra and XMM-Newton. For C V, N VI, and O VII, early reports indicated densities either around or below the low density limit, viz. $n_{e} \approx 10^{9}-10^{10} \mathrm{~cm}^{-3}$ for Capella (Brinkman et al. 2000; Canizares et al. 2000; Audard et al. 2001b; Mewe et al. 2001; Ness et al. 2001; Phillips et al. 2001), $\alpha$ Cen (Raassen et al. 2003a), Procyon (Ness et al. 2001; Raassen et al. 2002), HR 1099 (Audard et al. 2001a), and II Peg (Huenemoerder et al. 2001). Note that this sample covers an appreciable range of activity. (A conflicting, higher-density measurement, $n_{e} \approx[2-3] \times 10^{10} \mathrm{~cm}^{-3}$, was given by Ayres et al. 2001b for HR1099 and Capella). A low-density $\operatorname{limit}\left(\log n_{e}<10.2\right)$ is found for Capella also from the

Table 2. Density-sensitive He-like triplets ${ }^{\mathrm{a}}$

\begin{tabular}{llllll}
\hline Ion & $\lambda(r, i, f)(\AA)$ & $R_{0}$ & $N_{c}$ & $\log n_{e}$ range $^{\mathrm{b}}$ & $T$ range $^{\mathrm{c}}(\mathrm{MK})$ \\
\hline $\mathrm{Cv}$ & $40.28 / 40.71 / 41.46$ & 11.4 & $6 \times 10^{8}$ & $7.7-10$ & $0.5-2$ \\
$\mathrm{~N}_{\text {VI }}$ & $28.79 / 29.07 / 29.53$ & 5.3 & $5.3 \times 10^{9}$ & $8.7-10.7$ & $0.7-3$ \\
$\mathrm{O}_{\text {VII }}$ & $21.60 / 21.80 / 22.10$ & 3.74 & $3.5 \times 10^{10}$ & $9.5-11.5$ & $1.0-4.0$ \\
$\mathrm{Ne}_{\mathrm{IX}}$ & $13.45 / 13.55 / 13.70$ & 3.08 & $8.3 \times 10^{11}$ & $11.0-13.0$ & $2.0-8.0$ \\
$\mathrm{Mg}_{\text {XI }}$ & $9.17 / 9.23 / 9.31$ & $2.66^{\mathrm{d}}$ & $1.0 \times 10^{13}$ & $12.0-14.0$ & $3.3-13$ \\
$\mathrm{Si}$ XIII & $6.65 / 6.68 / 6.74$ & $2.33^{\mathrm{d}}$ & $8.6 \times 10^{13}$ & $13.0-15.0$ & $5.0-20$
\end{tabular}

${ }^{a}$ data derived from Porquet et al. (2001) at maximum formation temperature of ion

${ }^{b}$ range where $R$ is within approximately [0.1,0.9] times $R_{0}$

${ }^{c}$ range of $0.5-2$ times maximum formation temperature of ion

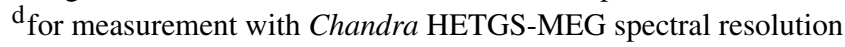


Ne IX triplet (Ness et al. 2003b). First unequivocal reports on significant, higher densities measured in O vil came for very active main-sequence stars such as AB Dor (Güdel et al. 2001b) and YY Gem (Güdel et al. 2001a), indicating $n_{e}$ of several times $10^{10} \mathrm{~cm}^{-3}$. The trend for higher densities in more active main-sequence stars is consistently found across various spectral types (Ness et al. 2002a; Raassen et al. 2003b; van den Besselaar et al. 2003), whereas active binaries may reveal either high or low densities (Ness et al. 2002b; Huenemoerder et al. 2001, 2003), and low-activity stars generally show low densities (Ness et al. 2002a). The most recent, comprehensive compilation of these trends can be found in Ness et al. (2004) who surveyed O VII and Ne IX triplets of a sample of 42 stellar systems across all levels of magnetic activity, and in Testa et al. (2004) who studied a sample of 22 stars with Chandra.

As for higher- $Z$ He-like triplets, reports become quite ambiguous, echoing both the results and the problems encountered in the analysis of Fe lines. Mewe et al. (2001) found $n_{e}=3 \times 10^{12} \mathrm{~cm}^{-3}-3 \times 10^{13} \mathrm{~cm}^{-3}$ in Capella from Mg XI and Si XIII as measured by the Chandra LETGS. These high values agree with EUVE measurements (e.g., Dupree et al. 1993), but they contradict simultaneous Chandra measurements obtained from Fe XX-XXII (Mewe et al. 2001). Osten et al. (2003) derived densities from He-like triplets, Fe XXI and Fe XXII line ratios over a temperature range of $\approx 1-15 \mathrm{MK}$. They found a sharply increasing trend: densities from lines formed below $6 \mathrm{MK}$ point at a modest electron density of a few times $10^{10} \mathrm{~cm}^{-3}$, while those formed above indicate densities exceeding $10^{11} \mathrm{~cm}^{-3}$, possibly reaching up to a few times $10^{12} \mathrm{~cm}^{-3}$. Somewhat perplexingly, though, the Si XIII triplet that is formed at similar temperatures as Mg XI suggests $n_{e}<10^{11} \mathrm{~cm}^{-3}$, and discrepancies of up to an order of magnitude become evident depending on the adopted formation temperature of the respective ion. The trend for an excessively high density implied by $\mathrm{Mg}$ can also be seen in the analysis of Capella by Audard et al. (2001b) and Argiroffi et al. (2003).

Clearly, a careful reconsideration of line blends is in order. Testa et al. (2004) have measured densities from $\mathrm{Mg}$ XI in a large stellar sample after modeling blends from $\mathrm{Ne}$ and Fe, still finding densities up to a few times $10^{12} \mathrm{~cm}^{-3}$ but not reaching beyond $10^{13} \mathrm{~cm}^{-3}$. All measurements from Si XIII imply an upper limit $\approx 10^{13} \mathrm{~cm}^{-3}$, casting some doubt on such densities derived from EUV Fe lines (see above). A trend similar to results from $\mathrm{O}$ VII is found again, namely that more active stars tend to reveal higher overall densities.

In the case of $\mathrm{Ne}$, the problematic situation with regard to line blends is illustrated in Fig. 12. If the density trend described above is real, however, then coronal loop pressures should vary by 3-4 orders of magnitude. This obviously requires different magnetic loop systems for the different pressure regimes, with a tendency that hotter plasma occupies progressively smaller volumes (Osten et al. 2003; Argiroffi et al. 2003).

Contrasting results have been reported, however. Canizares et al. (2000), Ayres et al. (2001b), and Phillips et al. (2001) found, from the Chandra HETG spectrum of Capella, densities at, or below the low-density limit for $\mathrm{Ne}, \mathrm{Mg}$, and $\mathrm{Si}$. A similar result applies to II Peg (Huenemoerder et al. 2001).

A summary of the present status of coronal density measurements necessarily remains tentative. Densities measured from Mg XI and Si XIII may differ greatly: despite their similar formation temperatures, $\mathrm{Mg}$ often results in very high densities, possibly induced by blends (see Testa et al. 2004); there are also discrepancies between densities 


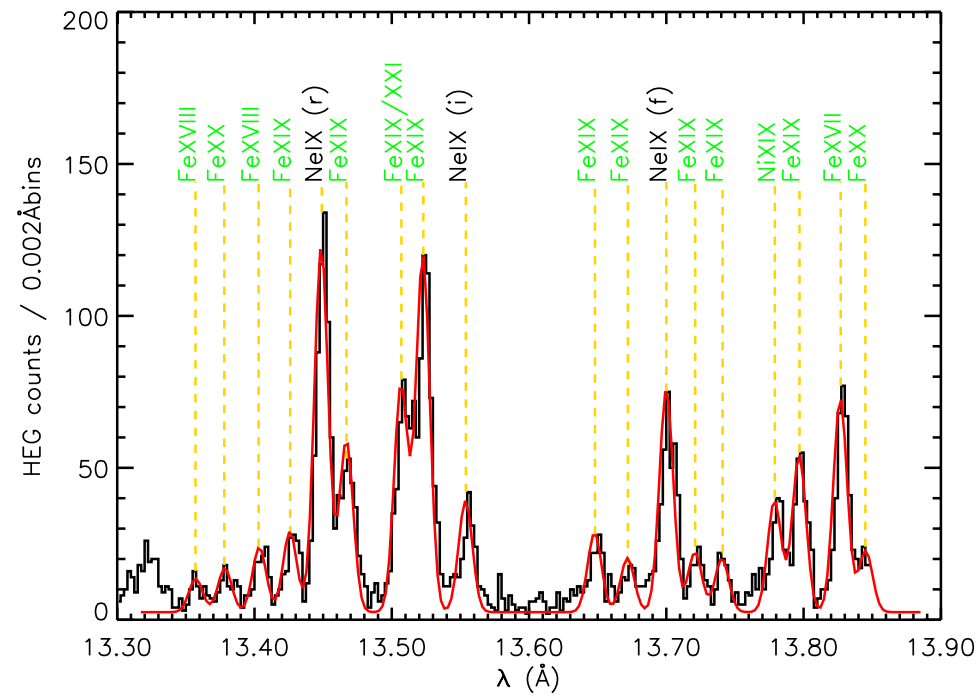

Fig. 12. The spectral region around the Ne IX triplet, showing a large number of Fe lines, some of which will blend with the Ne lines of interest if the resolving power is smaller than shown here (data from Chandra HETGS; the smooth red line shows a fit based on Gaussian line components; figure courtesy of J.-U. Ness)

derived from lines of He-like ions and from Fe XXI and Fe XXII line ratios, again for similar temperature ranges; and there is, lastly, disagreement between various authors who have used data from different instruments. The agreement is better for the cooler plasma components measured with $\mathrm{Cv}, \mathrm{N}$ VI, and $\mathrm{O}$ VII. There, inactive stars generally show $n_{e}<10^{10} \mathrm{~cm}^{-3}$, whereas densities of active stars may reach several times $10^{10} \mathrm{~cm}^{-3}$, values that are in fact in good agreement with measurements based on eclipses or rotational modulation (Sect. 11).

\subsection{Spectroscopic density measurements for inhomogeneous coronae}

Density measurements as discussed above have often been treated as physical parameters of an emitting source. However, because X-ray coronae are inhomogeneous, spectroscopic density measurements should not be taken at face value but should be further interpreted based on statistical models and distributions of coronal features. In spectroradiometry parlance, the observed irradiance, also loosely called "flux", from the stellar corona derives from the sum of the radiances over the entire visible coronal volume. Let us assume, for the sake of argument, an isothermal plasma around the maximum-formation temperature, $T$. Let us assume, further, that the emitting volumes, $V$, are distributed in electron density as a power-law, $d V / d n_{e} \propto n_{e}^{-\beta}$. We expect $\beta$ to be positive, i.e., low-density plasma occupies a large volume and high-density plasma is concentrated in small volumes. The densities inferred from the irradiance line ratios $R_{\text {obs }}$ of He-like ions are then biased toward the highest densities occurring in reasonably large volumes because of the $n_{e}^{2}$ dependence of the luminosity. 

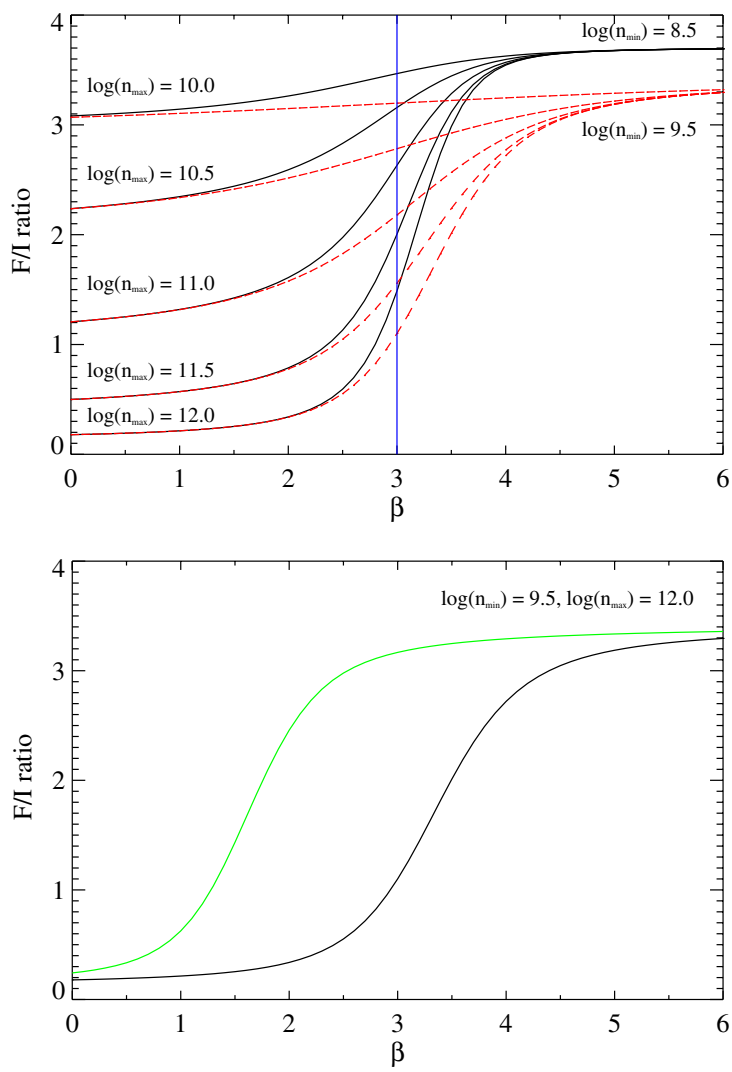

Fig. 13. Top: Calculated $R_{\mathrm{obs}}=F / I$ irradiance ratios for the He-like $\mathrm{O}$ VII line triplet originating in an inhomogeneous corona with a radiance distribution resulting from a power-law volumedensity distribution (see text for details). The $R$ ratio is plotted as a function of the power-law index $\beta$. The different curves are for different ranges of densities at which plasma is assumed to exist; the upper cutoff of the adopted range is given by the labels at left, while labels at right give the lower cutoff. The vertical line marks the transition from the low- $R$ to the high- $R$ regime at $\beta=3$. - Bottom: Example illustrating the $R_{\mathrm{obs}}=F / I$ irradiance ratio as a function of $\beta$ (black line), compared with the $R$ ratio that would correspond to an unweighted average of the densities of all volume elements considered (green). Atomic data from Porquet et al. (2001) were used

The observed forbidden and intercombination line irradiances $F$ and $I$ are the contributions of the radiances $f$ and $i$, respectively, integrated over the distribution $d V / d n_{e}$ and corrected for the stellar distance. The resulting observed $R_{\mathrm{obs}}=F / I$ ratios are plotted in Fig. 13a as a function of $\beta$ for reasonable ranges of coronal densities $\left[n_{e, \min }, n_{e, \max }\right]$ considered for the power-law volume distribution. The transition from low to high $R$ occurs around $\beta=3$, which is a direct consequence of the $n_{e}^{2}$ factor under the integral sign. A slight rearrangement of the distribution of active regions may thus dramatically change $R_{\mathrm{obs}}$. Further, again due to the $n_{e}^{2}$ dependence of the line flux, the "inferred density" is not an average over the coronal volume. In Fig. 13b, the spectroscopically measured $R$ ratios from this distribution are compared with the ratio that the linearly 
averaged density itself would produce (for one example). It illustrates that the inferred densities are considerably biased to higher values, that is, smaller $R$ ratios, for a given volume distribution (or $\beta$ ).

In a global coronal picture like that above, then, the $R_{\text {obs }}$ values do not describe "densities" but the steepness of the density distribution, and a straightforward interpretation of densities and volumes from the singular spectroscopic values can be misleading. The line ratio instead contains interesting information on the distribution of coronal densities. More active MS stars thus appear to maintain flatter density distributions, which could be a consequence of the finite coronal volume: since the latter must be shared by low- and high-density plasmas, the more efficient heating in more active stars (e.g., the increased production of chromospheric evaporation) produces a larger amount of high-density volume, at the cost of the residual low-density volume, thus flattening the distribution and decreasing $R$. Alternatively, as Fig. 13a illustrates, the cutoffs of the density distribution could be shifted to higher values for more active stars while the slope $\beta$ remains similar. The interpretation of coronal density measurements thus naturally connects to coronal structure, which is the subject of the following section.

\section{The structure of stellar coronae}

The magnetic structure of stellar coronae is one of the central topics in our research discipline. The extent and predominant locations of magnetic structures currently hold the key to our understanding of the internal magnetic dynamo. For example, compact or extended coronae may argue for or against the presence of a distributed dynamo. All X-ray inferences of coronal structure in stars other than the Sun are so far indirect. This section describes various methods to infer structure in stellar coronae at X-ray wavelengths, and reviews the results thus obtained.

\subsection{Loop models}

Closed magnetic loops are the fundamental "building blocks" of the solar corona. When interpreting stellar coronae of any kind, we assume that this concept applies as well, although caution is in order. Even in the solar case, loops come in a wide variety of shapes and sizes (Fig. 14) and appear to imply heating mechanisms and heating locations that are poorly understood - see, for example, Aschwanden et al. (2000a). Nevertheless, simplified loop models offer an important starting point for coronal structure studies and possibly for coronal heating diagnostics. A short summary of some elementary properties follows.

Under certain simplifying assumptions, loop scaling laws can be derived. These have been widely applied to stellar coronae. Rosner et al. (1978) (= RTV) have modeled hydrostatic loops with constant pressure (i.e., the loop height is smaller than the pressure scale height). They also assumed constant cross section, uniform heating, and absence of gravity, and found two scaling laws relating the loop semi-length $L$ (in $\mathrm{cm}$ ), the volumetric heating rate $\epsilon$ (in $\operatorname{erg~cm}^{-3} \mathrm{~s}^{-1}$ ), the pressure $p$ (in dynes $\mathrm{cm}^{-2}$ ), and the loop apex temperature $T_{\mathrm{a}}$ (in $\mathrm{K}$ ),

$$
T_{\mathrm{a}}=1400(p L)^{1 / 3} ; \quad \epsilon=9.8 \times 10^{4} p^{7 / 6} L^{-5 / 6} .
$$



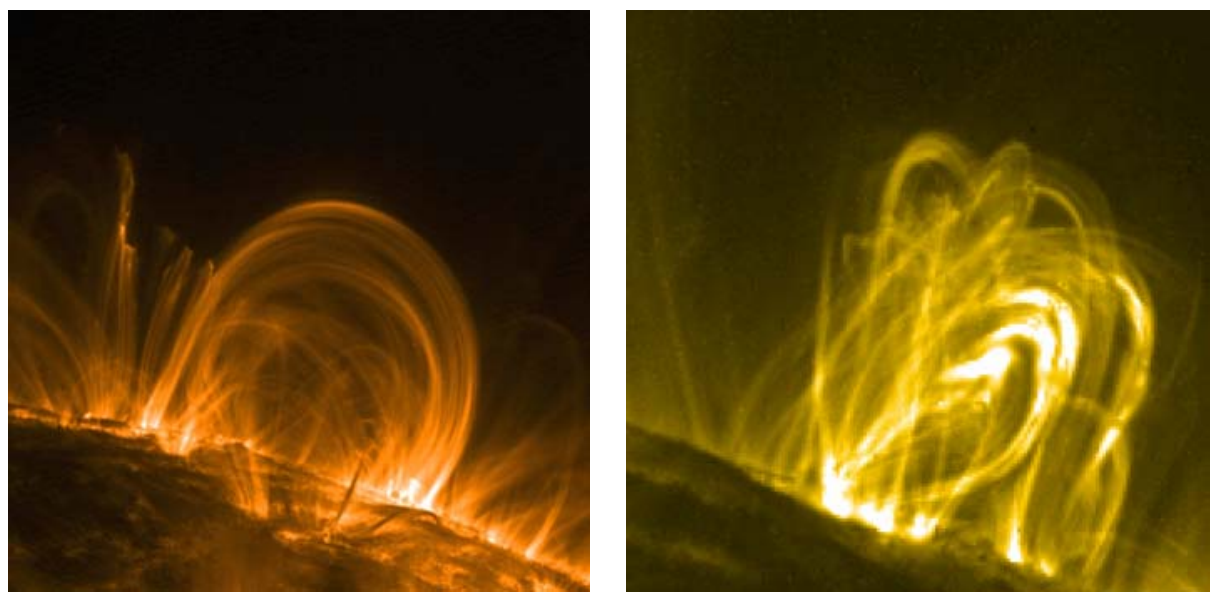

Fig. 14. Left: Example of a solar coronal loop system observed by TRACE. Right: Flaring loop system (observation by TRACE at $171 \AA$ ). Although these images show the emission from relatively cool coronal plasma, they illustrate the possible complexity of magnetic fields

Serio et al. (1981) extended these scaling laws to loops exceeding the pressure scale height $s_{p}$, whereby, however, the limiting height at which the loops grow unstable is $(2-3) s_{p}$ :

$$
T_{\mathrm{a}}=1400(p L)^{1 / 3} e^{-0.04 L\left(2 / s_{H}+1 / s_{p}\right)} ; \quad \epsilon=10^{5} p^{7 / 6} L^{-5 / 6} e^{0.5 L\left(1 / s_{H}-1 / s_{p}\right)} .
$$

Here, $s_{H}$ is the heat deposition scale height. For loops with an area expansion factor $\Gamma>1$, Vesecky et al. (1979) (= VAU) found numerical solutions that approximately follow the scaling laws (Schrijver et al. 1989b)

$$
T_{\mathrm{a}} \approx 1400 \Gamma^{-0.1}(p L)^{1 / 3} ; \quad T_{\mathrm{a}}=60 \Gamma^{-0.1} L^{4 / 7} \epsilon^{2 / 7} .
$$

Accurate analytical approximations to hydrostatic-loop solutions have been given by Aschwanden and Schrijver (2002) for uniform and non-uniform heating, including loops with expanding cross sections and loops heated near their footpoints. I note in passing that the hydrostatic equations allow for a second solution for cool loops $\left(T<10^{5} \mathrm{~K}\right)$ with essentially vanishing temperature gradients and small heights (on the Sun: $<5000 \mathrm{~km}$, the pressure scale height at $10^{5} \mathrm{~K}$ ). These are transition region loops that can be observed in the UV region (Martens and Kuin 1982; Antiochos and Noci 1986).

There are serious disagreements between some solar-loop observations and the RTV formalism so long as simplified quasi-static heating laws are assumed, the loops being more isothermal than predicted by the models. There is, however, only limited understanding of possible remedies, such as heating that is strongly concentrated at the loop footpoints, or dynamical processes in the loops (see, for example, a summary of this debate in Schrijver and Aschwanden 2002).

Unstable solutions of large coronal loops with temperature inversions at the loop apex were numerically studied by Collier Cameron (1988). If such loops are anchored on rapidly rotating stars, they may, in addition, become unstable under the influence of centrifugal forces. Once the latter exceed gravity, the pressure and the electron density 
grow outwards along the magnetic field, enhance the radiative loss rate and lead to a temperature inversion, which grows unstable. Rapid thermal cooling at the distant loop apex may then lead to condensations of prominence-like, magnetically trapped and centrifugally supported, synchronously rotating cold gas, for which there is indeed evidence at distances of $\approx 3 R_{*}$ around the rapid rotator AB Dor (Collier Cameron 1988). Further numerical studies, including various assumptions on the base pressure, surface magnetic field, and base conductive flux were presented by Unruh and Jardine (1997).

\subsection{Coronal structure from loop models}

\subsubsection{Loop parameters}

When we interpret stellar coronal spectra, we assume, to first order, that some physical loop parameters map on our measured quantities, such as temperature and EM (and possibly density), in a straightforward way. In the simplest approach, we assume that the observed luminosity $L_{X}$ is produced by an ensemble of identical coronal loops with characteristic half-length $L$, surface filling factor $f$, and an apex temperature $T$ used for the entire loop; then, on using (20) and identifying $L_{X}=\epsilon V$, we obtain

$$
L \approx 6 \times 10^{16}\left(\frac{R_{*}}{R_{\odot}}\right)^{2} \frac{f}{L_{X}} T^{3.5} \quad[\mathrm{~cm}] .
$$

This relation can only hold if $L$ is smaller than the pressure scale height. Based on this expression, the luminous, hot plasma component in magnetically active stars seems to invariably require either very large, moderate-pressure loops with a large filling factor, or solar-sized high-pressure compact loops with a very small $(<1 \%)$ filling factor (Giampapa et al. 1985; Stern et al. 1986; Schrijver et al. 1989b; Giampapa et al. 1996; Güdel et al. 1997a; Preibisch 1997a; Sciortino et al. 1999).

Schrijver et al. (1984) modeled $T$ and EM of a sample of coronal sources based on RTV loop models and found the following trends: i) Inactive MS stars such as the Sun are covered to a large fraction with large-scale, cool (2 MK) loops of modest size $\left(0.1 R_{*}\right)$. ii) Moderately active dwarfs are dominated by very compact, high-density, hot $(\approx 20 \mathrm{MK})$ loops that require large heating rates (up to 20 times more than for solar compact active region loops). iii) The most active stars may additionally form rather extended loops with heights similar to $R_{*}$.

\subsubsection{Loop-structure models}

While the above interpretational work identifies spectral-fit parameters such as $T$ or EMs with parameters of theoretical loop models, a physically more appealing approach involves full hydrostatic models whose calculated emission spectra are directly fitted to the observations. While physically more realistic than multi-isothermal models, the approach has its own limitations because it relies on a host of ad hoc parameters such as the location and distribution of heating sources within a loop, the loop geometry, the type of thermal conduction law inside the loop and, in particular, the unknown statistical distribution of all the loop parameters in a coronal ensemble. Fitting spectra calculated 
by use of full hydrostatic models permits, however, to constrain possible combinations of these physical loop parameters.

Giampapa et al. (1985) presented this type of numerical RTV-type loop models and, assuming that one type of loop dominates, fitted them both to stellar X-ray and transition-region UV fluxes. The modest success of the fitting suggested to them that their assumption was questionable and that various structures do coexist in stellar atmospheres. Stern et al. (1986) fitted numerical loop models, characterized by the loop apex temperature $T_{\mathrm{a}}$, the loop semi-length $L$, and the expansion factor $\Gamma$, to spectra from the Einstein Imaging Proportional Counter (IPC), and the solutions were again constrained by UV observations. The successful fit results indicated magnetic loops of modest size ( $L<10^{10} \mathrm{~cm}$ ) with modest filling factors $(f<10-20 \%)$, but with extreme, flare-like pressures ( $p \gtrsim 400$ dynes $\mathrm{cm}^{-2}$ ).

Giampapa et al. (1996) extended loop studies to M dwarfs observed by ROSAT. They found that the low- $T$ component at $\approx 1-2 \mathrm{MK}$ requires loops of small length $\left(L \ll R_{*}\right)$ but high pressure $\left(p>p_{\odot}\right)$, whereas the high- $T$ component at $\approx 5-10 \mathrm{MK}$ must be confined by rather long loops $\left(L \approx R_{*}\right)$ and high base pressures $(p \approx 20$ dynes $\left.\mathrm{cm}^{-2} \gg p_{\odot}\right)$ with filling factors of order 0.1 . These latter solutions however violate the applicability of the RTV scaling law, since the loop height exceeds the pressure scale-height. Giampapa et al. (1996) therefore speculated that this component is, in fact, related to multiple, very compact flaring regions with a small filling factor, while the cooler, compact component relates to non-flaring active regions.

The loop model approach has been extensively developed and further discussed in a series of papers by the Palermo group (Ciaravella et al. 1996, 1997; Maggio and Peres 1996, 1997; Ventura et al. 1998). The most notable results are:

1. To obtain a successful spectral fit to low-resolution data of a single loop, one usually requires at least two isothermal plasma components. The often-found two spectral components from fits to low-resolution spectra must not, in general, be identified with two loop families (Ciaravella et al. 1997).

2. Applications to observations often do, however, require two loop families in any case, but for reasons more involved than the presence of two dominant thermal components. In such cases, one finds relatively cool loops $(T=1.5-5 \mathrm{MK})$ with modest to high pressures $\left(p=2-100\right.$ dynes $\left.\mathrm{cm}^{-2}\right)$ and hot $(T=10-30 \mathrm{MK})$, extreme-pressure loops $\left(p=10^{2}-10^{4}\right.$ dynes $\left.\mathrm{cm}^{-2}\right)$. The latter are - once again - reminiscent of flaring loops with a very small surface filling factor (Maggio and Peres 1997; Ventura et al. 1998).

3. Low-activity stellar coronae, on the other hand, may be sufficiently well described by a single, dominant type of loop. This is the case for Procyon on which short, cool, low-pressure loops should occupy $10 \%$ of the surface in this picture.

Ottmann et al. (1993) found that the coronal structure on AR Lac can be interpreted with essentially one class of RTV loops with an apex temperature of $38 \mathrm{MK}$, although a better match may be based on variable cross-section VAU loops, an approach followed by Ottmann (1993), who found loops with a half-length $L \approx 3 \times 10^{11} \mathrm{~cm}$ and a filling factor $f \leq 10 \%$.

van den Oord et al. (1997) applied analytic loop models including non-zero conductive flux at the loop footpoints and variable expansion factors $\Gamma$ to $E U V E$ spectra. 
The spectral inversion is problematic because a given spectrum can be modeled by various sets of physical parameters, such as various combinations between loop expansion factors and loop base conductive fluxes. The range of solutions, however, consistently requires loop expansion factors of $2-5$, and in several stars, at least two loop families with different apex temperatures.

Sciortino et al. (1999) similarly applied tabulated loop models of steady-state coronal (RTV) loops to interpret medium-resolution X-ray spectra of M dwarfs. They again found that at least two classes of magnetic loops, one with an apex temperature of $\approx 10 \mathrm{MK}$ and one with several tens of $\mathrm{MK}$, are required. Although the solutions allow for a large range of base pressures and surface filling factors, both the cooler and the hotter loops are found to be quite compact, with lengths smaller than $0.1 R_{*}$ and filling factors of $10^{-4}$ to $10^{-3}$. A similar study was presented by Griffiths (1999) who computed comprehensive static energy-balance loop models to interpret EMDs of RS CVn binaries. The doublepeak structure in the EMD again required two loop families with apex temperatures of 8 and $22 \mathrm{MK}$, respectively, both of modest size.

Finally, Favata et al. (2000b) concluded, from modeling of several X-ray flares on AD Leo (Sect. 12.6), that all magnetic loops involved in the flaring corona are of similar, relatively compact dimension $\left(0.3 R_{*}\right)$. If these are the typical structures that form the overall corona, then their filling factor is no larger than about $6 \%$ despite the rather high X-ray luminosity of this star. The loop pressure must consequently be large (70 dyne $\mathrm{cm}^{-2}$ ).

\subsubsection{Conclusions and limitations}

The most essential conclusion from these exercises is perhaps that, within the framework of these simplistic models, the loop heating rate required for magnetically active stars may exceed values for typical solar loops by orders of magnitude, pointing toward some enhanced heating process reminiscent of the energy deposition in flares. The compactness of the hot loops and the consequent high pressures also set these coronal structures clearly apart from any non-flaring solar coronal features.

The inferred geometric size has important implications for dynamo theories. The apparent predominance of compact, localized sources suggests a predominance of local, small-scale magnetic fields. Such fields are expected from distributed dynamos that have been postulated for fully convective stars. A possible conclusion for deeply convective $\mathrm{M}$ dwarfs is that the solar-type $\alpha \omega$ dynamo does not operate, in agreement with the absence of a convective boundary inside the star once it becomes fully convective (Giampapa et al. 1996). This conclusion is, however, not entirely valid in the light of other magnetic field measurements that have revealed global components, e.g., by use of radio interferometry (Sect. 11.16). It is also important to note that open solar magnetic fields carry little specific EM, and that large-scale hot loops cannot be dense because the plasma cannot be magnetically confined in that case. Little X-ray emission is therefore expected from such features, even though they may well exist as magnetic structures.

While explicit loop models provide an appealing basis for a physical interpretation of coronal structures, the limitations of our diagnostics should be kept in mind:

1. due to the degeneracy of solutions in the product $p L$ (see (20)), multiple, largely differing solutions are usually compatible with observations of finite quality and of 
low energy resolution (e.g., Schmitt et al. 1985b for Procyon, van den Oord et al. 1997 for further sources);

2. the use of models based on one loop or one family of identical loops (in $L, T_{\mathrm{a}}$ and hence $p, f, \Gamma$, and the heating profile along the loop) may offer a number of degrees of freedom that is sufficiently large to describe a given spectrum satisfactorily, yet the model is unlikely to describe any real corona.

In this sense, like in the case of multi-isothermal models or in "minimum flux" coronal models, loop models parameterize the real situation in a manner that is not straightforward and that requires additional constraints from additional sources of information. I also refer to the extensive, critical discussion on this point by Jordan et al. (1986).

\subsection{Coronal structure from densities and opacities}

Spectroscopically measured densities provide, in conjunction with EMs, important estimates of emitting volumes. For example, Ness et al. (2001) use the RTV scaling laws together with measured coronal temperatures and electron densities inferred from Helike triplets of $\mathrm{C}, \mathrm{N}$, and $\mathrm{O}$ of Procyon. They concluded that the dominant coronal loops on this star are solar-like and low-lying $\left(L \lesssim 10^{9} \mathrm{~cm}\right)$. For the more active Capella, Mewe et al. (2001) found very similar structures covering a few tens of percent of the surface, but additionally they inferred the presence of a hotter plasma at apparently very high densities (Sect. 10.1). This latter plasma would have to be confined to within very compact loops $\left(L \lesssim 5 \times 10^{7} \mathrm{~cm}\right.$ ) that cover an extremely small area on the star $\left(f \approx 10^{-6}-10^{-4}\right)$. In general, for increasing temperature, progressively higher pressures and progressively smaller volumes are determined (Osten et al. 2003; Argiroffi et al. 2003; Sect. 10.2). The confinement of such exceedingly high densities in compact sources, with a size of a few $1000 \mathrm{~km}$, would then require coronal magnetic field strengths of order $1 \mathrm{kG}$ (Brickhouse and Dupree 1998). In that case, the typical magnetic dissipation time is only a few seconds for $n_{e} \approx 10^{13} \mathrm{~cm}^{-3}$ if the energy is derived from the same magnetic fields, suggesting that the small, bright loops light up only briefly. In other words, the stellar corona would be made up of numerous ephemeral loop sources that cannot be treated as being in a quasi-static equilibrium (van den Oord et al. 1997).

The debate as to how real these densities are, continues, as we mentioned in Sect. 10. Ness et al. (2002b), again using standard coronal-loop models, derived moderately compact loop sizes ( $L \approx 10^{9}-5 \times 10^{10} \mathrm{~cm}$ ) for Algol, the uncertainty being related to uncertain density measurements. Testa et al. (2004) and Ness et al. (2004) used density information from O VII, Ne IX, Mg XI, and computed a rough stellar surface filling factor $f$ of static loops. They found that $f$ remained at a few percent for cool, O vil emitting material for the most active stars. For less active stars, Ness et al. (2004) reported similar filling factors, whereas Testa et al. (2004) found them to decrease with decreasing activity. For the hotter, Mg XI emitting material, the surface coverage rapidly increases in more active stars. A possible interpretation involves a relatively cool, inactive base corona that remains unaltered while hotter and denser loops are added as one moves to progressively more active stars - a larger rate of magnetic interaction between adjacent active regions could then be assumed to heat the plasma, for example by flares.

The lack of measured optical depths $\tau$ due to resonance scattering in stellar coronae (see Sect. 15) can also be exploited to set limits to coronal size scales. Mewe et al. (2001) 
and Phillips et al. (2001) used formal upper limits on $\tau$ derived from Fe XVII line ratios of Capella together with densities derived from Fe XX-XXII line ratios (with the caveats mentioned above) to estimate that the characteristic size of a single emitting region is in the range of $(1-3) \times 10^{8} \mathrm{~cm}$, i.e., very compact in comparison with Capella's radius. This procedure, however, is not valid for more complicated geometries: if active regions are distributed across the stellar surface, then optical depths may cancel out in the observations, even if individual larger structures may be involved with non-zero optical depth (Mewe et al. 2001).

\subsection{Coronal constituents: Emission-measure interpretation}

The average X-ray surface flux $F_{X}$ is a direct tracer for the type of structures that can possibly cover the stellar surface. Because we generally have no information on stellar coronal inhomogeneities, the average stellar $F_{X}$ can obviously not be compared directly with $F_{X}$ values of solar coronal structures. We can nevertheless derive quite meaningful constraints. For example, the average $F_{X}$ on Proxima Centauri is only about one fifth of corresponding values for solar active regions while it exceeds solar quiet region values by one order of magnitude. Assuming, then, that the emission is concentrated in equivalents of solar active regions, their surface filling factor would be as much as $20 \%$, compared to $0.01-1 \%$ for the Sun at the 1 dyne $\mathrm{cm}^{-2}$ level (Haisch et al. 1980). At the low end of magnetic activity, there seem to be no stars with an X-ray surface flux below $F_{X} \approx 10^{4} \mathrm{erg} \mathrm{cm}^{-2} \mathrm{~s}^{-1}$ (Schmitt 1997). This flux coincides with the surface flux of solar coronal holes, suggesting that the least active MS stars are fully covered by coronal holes. Similar results were reported by Hünsch et al. (1996) for giants.

If coronal holes, inactive regions, active regions, bright points, small and large flares are characteristically different in their thermal structure and their surface flux, then one may interpret a full-disk EMD as a linear superposition of these various building blocks. We may start from the Sun by attempting to understand how various coronal features contribute to the integrated X-ray light (Ayres et al. 1996; Orlando et al. 2000, 2001; Peres et al. 2000). Full-disk solar EMDs from Yohkoh images reveal broad distributions with steep slopes below the temperature peak and a gradual decline up to $10^{7} \mathrm{~K}$. The interesting aspect is that the EMDs shift to higher temperatures both from activity minimum (peak at $\approx 1 \mathrm{MK}$ ) to maximum (peak at $\approx 2 \mathrm{MK}$ ) and from $\mathrm{X}$-ray faint to $\mathrm{X}$-ray bright features (see also Fig. 9).

On the stellar side, we see similar shifts from a hotter to a cooler EMD on evolutionary time scales as a star ages and becomes less active (Güdel et al. 1997a; Güdel 1997). For example, EMDs of intermediately active stars closely resemble the solar cycle-maximum EMD (Fig. 9), which indicates a nearly complete surface coverage with active regions (Drake et al. 2000). From this point of view, the Sun's magnetic cycle mimics an interval of stellar activity during its magnetic cycle (Ayres et al. 1996). Moving to very active stars, large-scale structures between active regions and post-flare loops seem to become the dominating coronal components rather than normal active regions and bright points. The increased luminosity is then a consequence of increased filling factors, increased loop base pressures, and higher temperatures (Ayres et al. 1996; Güdel et al. 1997a). Orlando et al. (2001) showed that in a similar manner the increasing X-ray spectral hardness from solar minimum to maximum on the one hand and from low-activity to 
high-activity solar analogs on the other hand can be explained by an increasing proportion of dense and bright coronal features, namely active regions and in particular hot cores of active regions.

However, if the Sun were entirely covered with active regions, the X-ray luminosity would amount to only $\approx(2-3) \times 10^{29} \mathrm{erg} \mathrm{s}^{-1}$ (Vaiana and Rosner 1978; Wood et al. 1994 ) with $L_{X} / L_{\text {bol }} \approx 10^{-4}$ (Vilhu 1984), short of $L_{X}$ of the most active early G-type analogs of the Sun by one order of magnitude. A similar problem is evident for extremely active, rapidly rotating FK Comae-type giants whose full disk $F_{X}$ exceeds non-flaring solar active region fluxes by up to an order of magnitude (Gondoin et al. 2002; Gondoin 2003a). In those stars, the cooler $(<10 \mathrm{MK})$ plasma component alone already fills the complete surface under the usual assumptions.

The DEMs of such active stars produce excessive emission around 10-20 MK (Güdel et al. 1997a), which incidentally is the typical range of solar flare temperatures. This led to the suggestion that the high- $T$ DEM is in fact due to the superposition of a multitude of superimposed but temporally unresolved flares (Sect. 13). If added to a low- $T$ "quiescent" solar DEM, the time-integrated DEM of solar flares indeed produces a characteristic bump around 10-20 MK that compares favorably with stellar DEMs (Güdel et al. 1997a). This happens because the flare EM decreases rapidly as the temperature decays, leaving a trace on the DEM only at relatively high temperatures, in agreement with (12) for time-integrated flares. If a full distribution of flares contributes, including small flares with lower temperature, then the entire DEM could be formed by the continually heating and cooling plasma in flares (Güdel 1997). The predicted steep low- $T$ slope (up to $\approx 4$ ) of a stochastic-flare DEM compares very favorably with observations of active stars (Güdel et al. 2003a).

The reason for an increased fraction of hotter features in more active stars (Fig. 10) may be found in the coronal structure itself. Toward more active stars, magnetic fields interact progressively more frequently due to their denser packing. In this view, an increased heating rate in particular in the form of flares reflects the enhanced dynamo operation in rapidly rotating stars. Since flares enhance the electron density along with the temperature, one finds a predominance of hotter structures in more active stars (Güdel et al. 1997a; cf. also the discussion in Sect. 9.5). Along these lines, Phillips et al. (2001) compared high-resolution spectra of Capella and solar flares, finding a surprising overall agreement; similarly, Güdel et al. (2004) compared the average X-ray spectrum of a large stellar flare with the spectrum of low-level emission of the very active dMe binary YY Gem. The agreement between the spectral features, or equivalently, the EMD, is compelling. The hypothesis of ongoing flaring in active stars that we have now repeatedly invoked will be further discussed in Sect. 13.

\subsection{Coronal imaging: Overview}

As the development of solar coronal physics has amply shown, coronal imaging will be an indispensable tool for studying the structure and heating of stellar coronae in detail. At present, coronal structure recognition based on direct or indirect imaging methods is still highly biased by observational constraints and by the location of physical processes that heat plasma or accelerate particles. Stellar coronal structure resolved at radio wavelengths refers to extended (typically low-density) closed magnetic fields into 
which high-energy electrons have been injected. X-ray emitting plasma traces magnetic fields that have been loaded with dense plasma. But both types of structure, as well as coronal holes that are weak both in X-rays and at radio wavelengths, refer to the underlying global distribution of surface magnetic fields. Presently, only radio structures can be directly imaged by means of radio Very Long Baseline Interferometry (VLBI; for a review see Güdel 2002), whereas thermal coronal sources can be mapped indirectly by using X-ray eclipses, rotational modulation, or Doppler information as discussed below.

With these limitations in mind, I now briefly summarize various image reconstruction methods for eclipses and rotational modulation (Sects. 11.6-11.9) and related results (Sects. 11.10-11.13). Sections 11.14 and 11.15 address alternative methods for structure modeling.

In its general form, the "image" to be reconstructed consists of volume elements at coordinates $(x, y, z)$ with optically thin fluxes $f(x, y, z)$ assumed to be constant in time. In the special case of negligible stellar rotation during the observation, the problem can be reduced to a 2-D projection onto the plane of the sky, at the cost of positional information along the line of sight (Fig. 15). In general, one thus seeks the geometric brightness distribution $f(x, y, z)=f_{i j k}(i, j, k$ being the discrete pixel number indices) from a binned, observed light curve $F_{s}=F\left(t_{s}\right)$ that undergoes a modulation due to an eclipse or due to rotation.

\subsection{Active-region modeling}

In the most basic approach, the emitting X-ray or radio corona can be modeled by making use of a small number of simple, elementary building blocks that are essentially described by their size, their brightness, and their location. This approach is the 3-D equivalent to standard surface spot modeling. Preferred building block shapes are radially directed, uniformly bright, optically thin, radially truncated spherical cones with their apexes at the stellar center. Free parameters are their opening angles, their heights above the stellar surface, their radiances, and their central latitudes and longitudes. These parameters are then varied until the model fits the observed light curve. For extensive applications to eclipsing binaries, see, for example, White et al. (1990), Ottmann (1993), and Culhane et al. (1990).

A minimum solution was presented by Güdel and Schmitt (1995) for a rotationally modulated star. If a rotationally modulated feature is invisible during a phase interval $\varphi$ of the stellar rotation, then all sources contributing to this feature must be confined to within a maximum volume, $V_{\max }$, given by

$$
\frac{V_{\max }}{R_{*}^{3}}=\frac{\psi}{3}-\frac{(2 \pi-\varphi)\left(1+\sin ^{2} i\right)}{6 \sin i}+\frac{2 \cot (\chi / 2)}{3 \tan i}
$$

where $\tan \chi=\tan (\varphi / 2) \cos i, \sin (\psi / 2)=\sin (\varphi / 2) \sin i$ with $0 \leq \psi / 2 \leq \pi / 2$, and $\chi$ and $\varphi / 2$ lie in the same quadrant ( $i$ is the stellar inclination, $0 \leq i \leq \pi / 2$ ). Together with the modulated fraction of the luminosity, lower limits to average electron densities in the modulated region follow directly. 


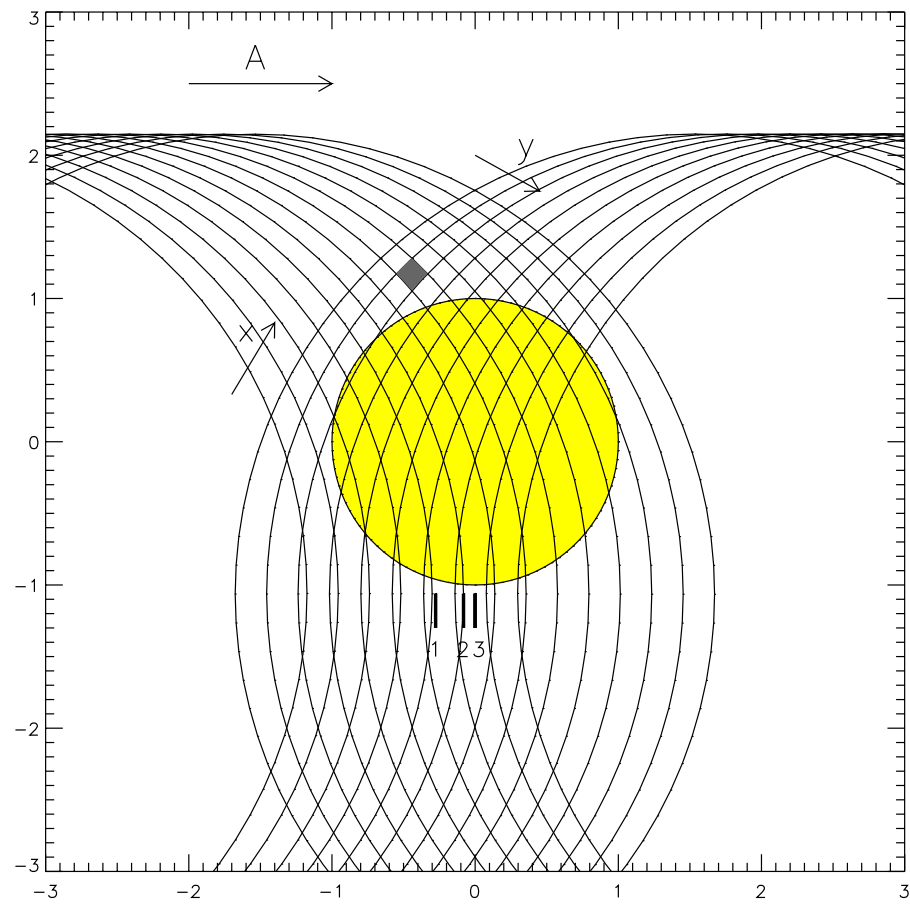

Fig. 15. Sketch showing the geometry of an eclipsing binary (in this case, $\alpha \mathrm{CrB}$, figure from Güdel et al. 2003b). The large circles illustrate the limbs of the eclipsing star that moves from left to right in front of the eclipsed star (shown in yellow). The limbs projected at different times during ingress and egress define a distorted 2-D array $(x, y)$ of pixels (an example of a pixel is shown in gray)

\subsection{Maximum-entropy image reconstruction}

We follow the outline given in Güdel et al. (2003b) to discuss maximum entropy methods (MEM) for image reconstruction. They are applicable both to rotationally modulated light curves and to eclipse observations. The standard MEM selects among all images $f_{i j k}$ (defined in units of counts per volume element) that are compatible with the observation, the one that minimizes the Kullback contrast ("relative entropy")

$$
K=\sum_{i, j, k} f_{i j k} \ln \frac{f_{i j k}}{f_{i j k}^{a}}
$$

with respect to an a priori image $f_{i j k}^{a}$, which is usually unity inside the allowed area or volume and vanishes where no brightness is admitted. Minimizing $K$ thus introduces the least possible information while being compatible with the observation. The contrast $K$ is minimum if $f_{i j k}$ is proportional to $f_{i j k}^{a}$ and thus flat inside the field of view, and it is maximum if the whole flux is concentrated in a single pixel $(i, j, k)$. The compatibility with the observed count light curve is measured by $\chi^{2}$,

$$
\chi^{2}=\sum_{s} \frac{\left(F_{s}^{*}-F_{s}\right)^{2}}{F_{s}^{*}}
$$


where $F_{s}$ and $F_{s}^{*}$ are, respectively, the observed number of counts and the number of counts predicted from $f_{i j k}$ and the eclipse geometry. Poisson statistics usually requires more than 15 counts per bin. Finally, normalization is enforced by means of the constraint

$$
N=\frac{\left(f^{\text {tot }}-\sum_{i j k} f_{i j k}\right)^{2}}{f^{\text {tot }}}
$$

where $f^{\text {tot }}$ is the sum of all fluxes in the model.

The final algorithm minimizes the cost function

$$
C=\chi^{2}+\xi K+\eta N .
$$

The trade-off between the compatibility with the observation, normalization, and unbiasedness is determined by the Lagrange multipliers $\xi$ and $\eta$ such that the reduced $\chi^{2}$ is $\lesssim 1$, and normalization holds within a few percent. For applications of this method and variants thereof, see White et al. (1990) and Güdel et al. (2003b).

\subsection{Lucy/Withbroe image reconstruction}

This method (after Lucy 1974 and Withbroe 1975, the latter author discussing an application to spectral line flux inversion) was extensively used by Siarkowski (1992), Siarkowski et al. (1996), Preś et al. (1995), Güdel et al. (2001a), Güdel et al. (2003b) and Schmitt et al. (2003) to image X-ray coronae of eclipsing binaries; it is applicable to pure rotational modulation as well. The method is formally related to maximum likelihood methods although the iteration and its convergence are methodologically different (Schmitt 1996). The algorithm iteratively adjusts fluxes in a given set of volume elements based on the mismatch between the model and the observed light curves in all time bins to which the volume element contributes. At any given time $t_{s}$ during the eclipse, the observed flux $F_{S}$ is the sum of the fluxes $f_{i j k}$ from all pixels that are unocculted:

$$
F\left(t_{s}\right)=\sum_{i, j, k} f_{i j k} \mathbf{m}_{s}(i, j, k)
$$

where $\mathbf{m}_{s}$ is the "occultation matrix" for the time $t_{s}$ : it puts, for any given time $t_{s}$, a weight of unity to all visible pixels and zero to all invisible pixels (and intermediate values for partially occulted pixels). Since $F_{S}$ is given, one needs to solve (29) for the flux distribution, which is done iteratively as follows:

$$
f_{i j k}^{n+1}=f_{i j k}^{n} \frac{\sum_{s} \frac{F_{o}\left(t_{s}\right)}{F_{m}^{n}\left(t_{s}\right)} \mathbf{m}_{s}(i, j, k)}{\sum_{s} \mathbf{m}_{s}(i, j, k)}
$$

where $F_{o}\left(t_{s}\right)$ and $F_{m}^{n}\left(t_{s}\right)$ are, respectively, the observed flux and the model flux (or counts) in the bin at time $t_{s}$, both for the iteration step $n$. Initially, a plausible, smooth distribution of flux is assumed, e.g., constant brightness, or some $r^{-p}$ radial dependence. 


\subsection{Backprojection and Clean image reconstruction}

If rotation can be neglected during an eclipse, for example in long-period detached binaries, then the limb of the eclipsing star is projected at regular time intervals onto the plane of the sky and therefore onto a specific part of the eclipsed corona, first during ingress, later during egress. The two limb sets define a 2-D grid of distorted, curved pixels (Fig. 15). The brightness decrement during ingress or, respectively, the brightness increment during egress within a time step $\left[t_{s}, t_{s+1}\right]$ originates from within a region confined by the two respective limb projections at $t_{s}$ and $t_{s+1}$. Ingress and egress thus each define a 1-D image by backprojection from the light curve gradients onto the plane of the sky. The relevant reconstruction problem from multiple geometric projections is known in tomography. The limiting case of only two independent projections can be augmented by a CLEAN step, as follows. The pixel with the largest sum of projected fluxes from ingress and egress is assumed to represent the location of a real source. A fraction, $g<1$, of this source flux is then subtracted from the two projections and saved on a clean map, and the process is iterated until all flux is transferred onto the latter. This method was described by Güdel et al. (2003b) who applied it to a total stellar eclipse of $\alpha \mathrm{CrB}$, with consideration of different gain factors $g$ and post-fit flux redistributions to study multiple solutions.

\subsection{Coronal structure inferred from eclipses}

I now review selected results from analyses of X-ray eclipses, and, in subsequent subsections, of rotational modulation and Doppler measurements. Some important parameters are summarized in Table 3.

\subsubsection{Extent of eclipsed features}

Some shallow X-ray eclipses in tidally interacting binary systems of the RS CVn, Algol, or BY Dra type have provided important information on extended coronal structure. For example, Walter et al. (1983) concluded that the coronae in the AR Lac binary components are bi-modal in size, consisting of compact, high-pressure (i.e., 50-100 dynes $\mathrm{cm}^{-2}$ ) active regions with a scale height $<R_{*}$, while the subgiant $\mathrm{K}$ star is additionally surrounded by an extended $\left(2.7 R_{*}\right.$ ) low-pressure corona. This view was supported by an analysis of ROSAT data by Ottmann et al. (1993). In the analysis of White et al. (1990), the association of different regions with the binary components remained ambiguous, and so did the coronal heights, but the most likely arrangement again required at least one compact region with $p>100$ dynes $\mathrm{cm}^{-2}$ and favored an additional extended, low-pressure coronal feature with $p \approx 15$ dynes $\mathrm{cm}^{-2}$ and a scale height of $\approx R_{*}$. Further, a hot component pervading the entire binary system was implied from the absence of an eclipse in the hard ME detector on EXOSAT. Culhane et al. (1990) similarly observed a deep eclipse in TY Pyx in the softer band of EXOSAT but a clear absence thereof in the harder band, once more supporting a model including an extended, hot component. From an offset of the eclipse relative to the optical first contact in Algol, Ottmann (1994) estimated the height of the active K star corona to be $\approx 2.8 R_{\odot}$. 
Table 3. X-ray coronal structure inferred from eclipses and rotational modulation

\begin{tabular}{|c|c|c|c|c|c|c|}
\hline Star & Spectrum & $\begin{array}{l}\text { Extended }^{\mathrm{a}} \\
\text { height }\left(R_{*}\right)\end{array}$ & $n_{e}^{\mathrm{b}}$ & $\begin{array}{l}\text { Compact }^{\mathrm{a}} \\
\text { height }\left(R_{*}\right)\end{array}$ & $n_{e}^{\mathrm{b}}$ & Reference $^{c}$ \\
\hline AR Lac & $\mathrm{G} 2 \mathrm{IV}+\mathrm{K} 0 \mathrm{IV}$ & $\approx 1$ & 0.29 & 0.01 & $4-6$ & 1 \\
\hline AR Lac & G2 IV+K0 IV & $1.1-1.6$ & $0.2-0.8$ & 0.06 & $>5$ & 2 \\
\hline AR Lac & G2 IV+K0 IV & $0.7-1.4$ & $0.3-0.8$ & $0.03-0.06$ & $6-60$ & 3 \\
\hline AR Lac & $\mathrm{G} 2 \mathrm{IV}+\mathrm{K} 0 \mathrm{IV}$ & $\approx 1$ & $0.12-1.8$ & - & - & 4 \\
\hline Algol & $\mathrm{B} 8 \mathrm{~V}+\mathrm{K} 2 \mathrm{IV}$ & 0.8 & $\ldots$ & - & - & 5 \\
\hline Algol $^{\mathrm{d}}$ & $\mathrm{B} 8 \mathrm{~V}+\mathrm{K} 2 \mathrm{IV}$ & - & - & $\lesssim 0.5$ & $\gtrsim 9.4$ & 6 \\
\hline Algol $^{\mathrm{d}}$ & $\mathrm{B} 8 \mathrm{~V}+\mathrm{K} 2 \mathrm{IV}$ & - & - & 0.1 & $\lesssim 3$ & 7 \\
\hline TY Pyx & G5 IV+G5 IV & $\approx 1-2$ & $0.02-3$ & - & - & 8 \\
\hline XY UMa & $\mathrm{G} 3 \mathrm{~V}+\mathrm{K} 4 \mathrm{~V}$ & - & - & $\leq 0.75$ & $\ldots$ & 9 \\
\hline VW Cep ${ }^{d}$ & K0 V+G5 V & 0.84 & 5 & - & - & 10 \\
\hline$\alpha \mathrm{CrB}$ & $\mathrm{A} 0 \mathrm{~V}+\mathrm{G} 5 \mathrm{~V}$ & - & - & $\lesssim 0.2$ & $\lesssim 3$ & 11 \\
\hline$\alpha \mathrm{CrB}$ & $\mathrm{A} 0 \mathrm{~V}+\mathrm{G} 5 \mathrm{~V}$ & - & - & $\lesssim 0.1$ & $0.1-3$ & 12 \\
\hline EK Dra & dG0e & - & - & $\lesssim 0.2$ & $\gtrsim 4$ & 13 \\
\hline YY Gem & $\mathrm{dM} 1 \mathrm{e}+\mathrm{dM} 1 \mathrm{e}$ & - & - & $0.25-1$ & $0.3-3$ & 14 \\
\hline V773 Tau ${ }^{\mathrm{d}}$ & $\mathrm{K} 2 \mathrm{~V}+\mathrm{K} 5 \mathrm{~V}$ & & & $\approx 0.6$ & $\geq 20$ & 15 \\
\hline
\end{tabular}

Notes. ${ }^{\text {a }}$ Extended structures of order $R_{*}$, compact structures significantly smaller.

${ }^{\mathrm{b}}$ Electron density in $10^{10} \mathrm{~cm}^{-3}$ for extended and compact structures, respectively.

${ }^{c}$ References: 1 Walter et al. (1983); 2 White et al. (1990); 3 Ottmann et al. (1993); 4 Siarkowski et al. (1996); 5 Ottmann (1994); 6 Schmitt and Favata (1999); 7 Schmitt et al. (2003); 8 Preś et al. (1995); 9 Bedford et al. (1990); 10 Choi and Dotani (1998); 11 Schmitt and Kürster (1993); 12 Güdel et al. (2003b); 13 Güdel et al. (1995); 14 Güdel et al. (2001a); Skinner et al. (1997).

${ }^{\mathrm{d}}$ Refers to observation of eclipsed/modulated flare.

White et al. (1986) inferred, from the absence of X-ray dips or any modulation in the light curve of Algol, a minimum characteristic coronal scale height of $3 R_{\odot}$ (i.e., about $1 R_{*}$ ). Similar arguments were used by Jeffries (1998) for the short period system XY UMa to infer a corona that must be larger than $1 R_{\odot}$ unless more compact structures sit at high latitudes. This latter possibility should in fact be reconsidered for several observations that entirely lack modulation. Eclipses or rotational modulation can be entirely absent if the active regions are concentrated toward one of the polar regions. This possibility has found quite some attention in recent stellar research.

Detailed studies of light curves that cover complete binary orbits with ASCA raise, however, some questions on the reliability of the derived structure sizes. Unconstrained iterations of the light curve inversion algorithm do converge to structures that are extended on scales of $R_{*}$ (Fig 16); but constrained solutions exist that sufficiently represent the light curves with sources no larger than $0.3 R_{*}$ (Siarkowski et al. 1996). Nevertheless, detailed studies of the imaging reconstruction strategy and the set-up of initial conditions led Preś et al. (1995) to conclude that X-ray bright sources do indeed exist far above the surfaces in the TY Pyx system, most likely located between the two components. The latter configuration includes the possibility of magnetic fields connecting the two stars. Interconnecting magnetic fields would draw implications for magnetic heating through 

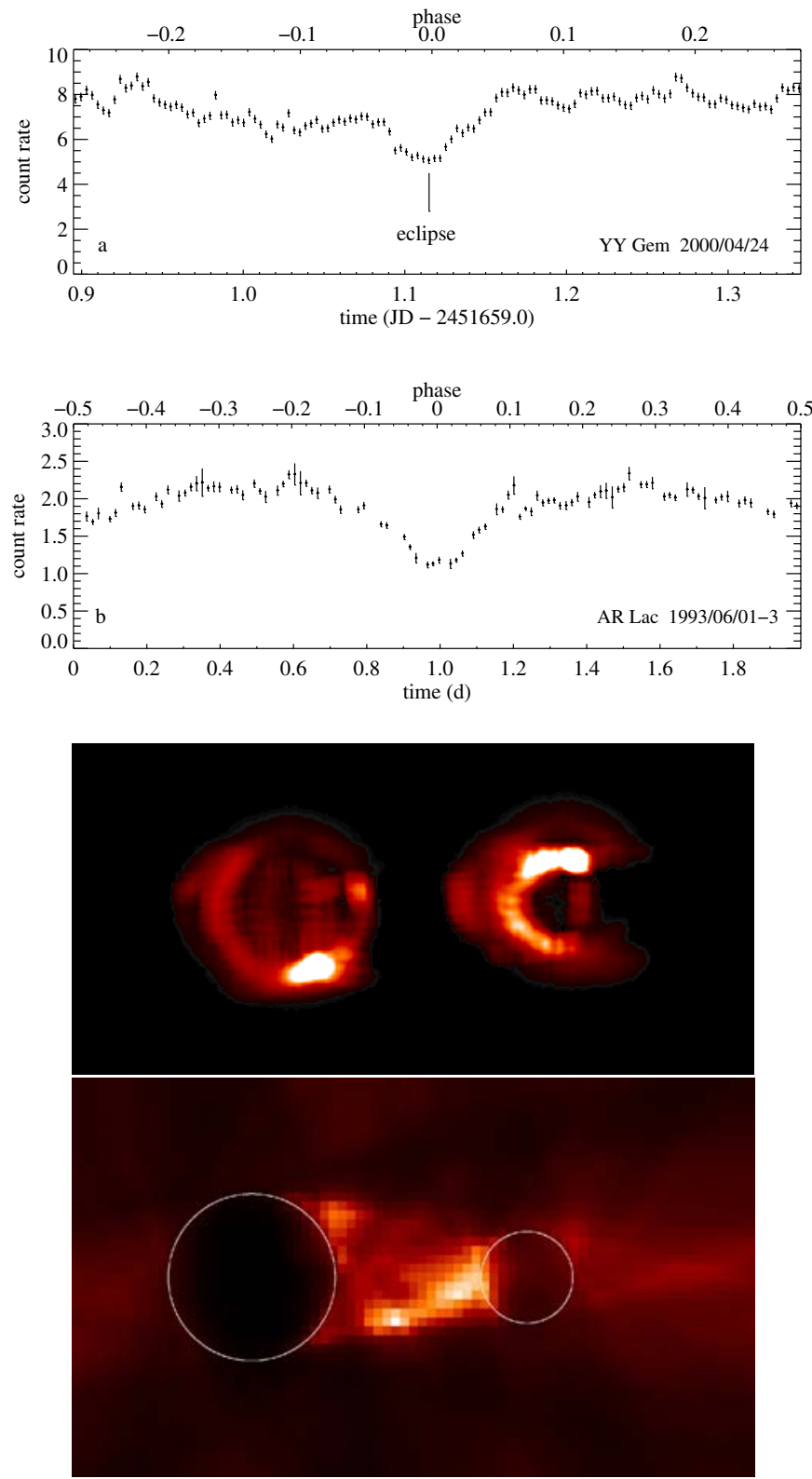

Fig. 16. Two examples of eclipses and the corresponding coronal image reconstructions. From top to bottom: Light curve of the YY Gem system (from Güdel et al. 2001a, observation with XMM-Newton EPIC); light curve of the AR Lac system (after Siarkowski et al. 1996, observation with ASCA SIS); reconstructed image of the coronal structure of, respectively, YY Gem (at phase 0.375 ) and AR Lac (at quadrature). The latter figure shows a solution with intrabinary emission. (The light curve of AR Lac is phase-folded; the actual observation started around phase 0; data and image for AR Lac courtesy of M. Siarkowski.) 
reconnection between intrabinary magnetic fields, as was suggested by Uchida and Sakurai $(1984,1985)$ and was also proposed from radio observations of the RS CVn-type binary CF Tuc (Gunn et al. 1997), the Algol-type binary V505 Sgr (Gunn et al. 1999), and the pre-cataclysmic binary V471 Tau (Lim et al. 1996). The X-ray evidence remains ambiguous at this time, and alternative X-ray methods such as Doppler measurements (Ayres et al. 2001b) have not added support to this hypothesis (Sect. 11.14).

\subsubsection{Structure and location of coronal features}

The eclipse light curves often require asymmetric, inhomogeneous coronae, with bright features sometimes found on the leading stellar hemispheres (e.g., Walter et al. 1983; Ottmann et al. 1993; Ottmann 1994), but often also on the hemispheres facing each other (Bedford et al. 1990; Culhane et al. 1990; White et al. 1990; Siarkowski 1992; Siarkowski et al. 1996; Preś et al. 1995). This may, as mentioned above, have important implications for intrabinary magnetic fields. For the dMe binary YY Gem, Doyle and Mathioudakis (1990) reported a preferred occurrence of optical flares also on the two hemispheres facing each other, and this hypothesis has been supported by the timing of Xray flares (Haisch et al. 1990b). However, X-ray image reconstruction from light curves does not require any emission significantly beyond $2 R_{*}$ (Güdel et al. 2001a, Fig. 16): interconnecting magnetic fields are thus not supported in this case. Similarly, a deep eclipse observed on XY UMa (in contrast to an observation reported by Jeffries 1998) places the eclipsed material on the hemisphere of the primary that faces the companion, but judged from thermal loop models, the sources are suggested to be low-lying (Bedford et al. 1990). A concentration of activity on the inner hemispheres could, alternatively, be induced by tidal interactions and may therefore not require any interconnecting magnetic fields (Culhane et al. 1990).

Eclipse modulation also confines the latitudes $b$ of the eclipsed material. For AR Lac, $b=10^{\circ}-40^{\circ}$ (Walter et al. 1983, and similarly in White et al. 1990, and Ottmann et al. 1993). Bedford et al. (1990) infer $-30^{\circ} \leq b \leq+30^{\circ}$ from a deep eclipse on XY UMa. Most active regions on the dMe binary YY Gem (dM1e+dM1e) are concentrated around $\pm\left(30^{\circ}-50^{\circ}\right)$ (Güdel et al. 2001a), in good agreement with Doppler imaging of surface active regions (Hatzes 1995). The confinement of the inhomogeneities leads to the somewhat perplexing result that these most active stars reveal "active" X-ray filling factors of no more than 5-25\% despite their being in the saturation regime (Sect. 5; see White et al. 1990; Ottmann et al. 1993).

\subsubsection{Thermal properties of coronal structures}

The presence of distinct compact and extended coronae may reflect the presence of different thermal structures - in fact, the distinction may simply be due to different scale heights if magnetic fields do not constrain the corona further. The average radial density profile of YY Gem with a scale height of $\approx[0.1-0.4] R_{*}$ derived from eclipse reconstruction is in good agreement with the pressure scale height of the one component of the plasma that dominates the X-ray spectrum (Güdel et al. 2001a). Compact sources are often inferred with size scales comparable to solar active regions. Based on such arguments, the extended structures are more likely to be associated with the hottest persistent plasma in active binaries (Walter et al. 1983; White et al. 1990; Rodonò et 
al. 1999, but see Singh et al. 1996a and Siarkowski et al. 1996 for alternative views). Inferred pressures of up to $>100$ dynes $\mathrm{cm}^{-2}$ make these regions appear like continuously flaring active regions (Walter et al. 1983; White et al. 1990). Alternatively, they could contain low-density, slowly cooling gas ejected from large flares. This view would be consistent with radio VLBI observations. The latter have mapped non-thermal flares that expand from compact cores to extended $\left(\gg 1 R_{*}\right)$ halos where they cool essentially by radiation (Güdel 2002 and references therein). Conjecture about different classes of thermal sources is again not unequivocal, however. Ottmann et al. (1993) and Ottmann (1994) found equivalent behavior of soft and hard spectral components during eclipses in AR Lac and Algol, respectively, and argued in favor of a close spatial association of hot and cool coronal components regardless of the overall spatial extent. This issue is clearly unresolved.

\subsection{X-ray coronal structure in other eclipsing binaries}

Among wide, non-interacting eclipsing stars, $\alpha \mathrm{CrB}$ provides a particularly well-suited example because its X-ray active, young solar analog (G5 V) is totally eclipsed every 17 days by the optical primary, an A0 V star that is perfectly X-ray dark. Other parameters are ideal as well, such as the non-central eclipse, the eclipse time-scale of a few hours, and the relatively slow rotation period of the secondary. Eclipse observations obtained by ROSAT (Schmitt and Kürster 1993) and by XMM-Newton (Güdel et al. 2003b) were used to reconstruct projected 2-D images of the X-ray structure. They consistently reveal patches of active regions across the face of the $\mathrm{G}$ star; not much material is found significantly beyond its limb (Fig. 17). The structures tend to be of modest size $\left(\approx 5 \times 10^{9} \mathrm{~cm}\right)$, with large, X-ray faint areas in between, although the star's luminosity exceeds that of the active Sun by a factor of $\approx 30$. These observations imply moderately high densities in the emitting active regions, and both studies mentioned above yielded average electron densities in the brightest active regions of a few $10^{10} \mathrm{~cm}^{-3}$.
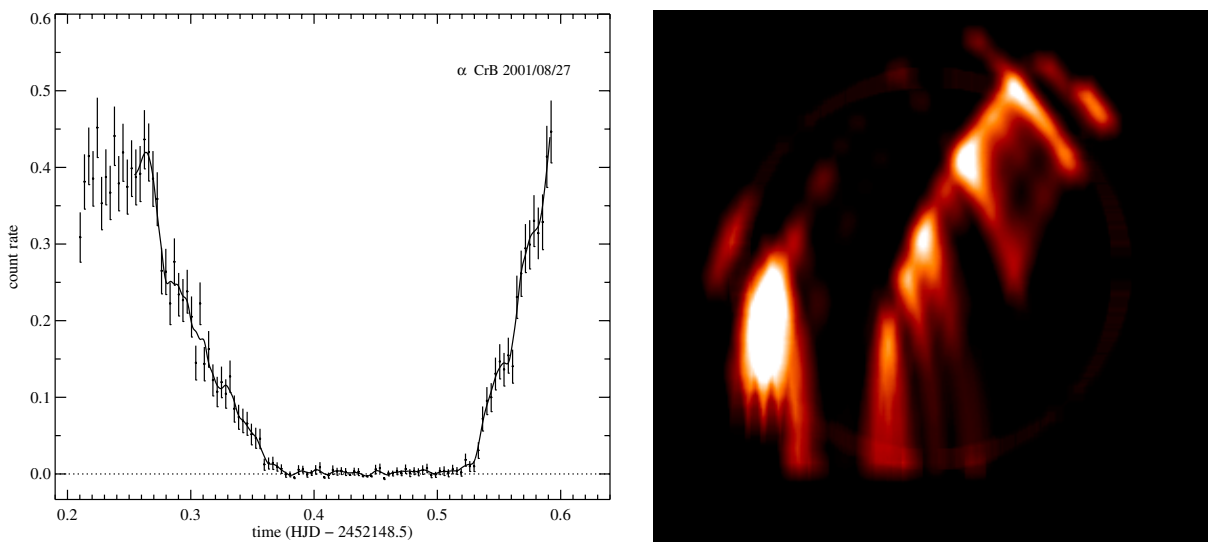

Fig. 17. Light curve and image reconstruction of the $\mathrm{A}+\mathrm{G}$ binary $\alpha \mathrm{CrB}$. The left panel shows the light curve from observations with $X M M$-Newton, the right panel illustrates the reconstructed X-ray brightness distribution on the G star (after Güdel et al. 2003b) 
X-ray light curves of eclipsing contact binary systems have shown sharp dips (Vilhu and Heise 1986; Gondoin 2004a) that could be interpreted as being due to compact sources probably located in the "neck" region that connects the two stars (Vilhu and Heise 1986) although Gondoin (2004a) inferred quite an extended corona from static loop models. Brickhouse and Dupree (1998), in contrast, placed a very compact source, with an extent of order $10^{8} \mathrm{~cm}$, near the polar region of the primary, complemented by a more extended low-density corona that contributes most of the X-ray light.

\subsection{Inferences from rotational modulation}

The Sun often shows rather pronounced rotational modulation in X-rays as few active regions rotate into and out of view. Observations of X-ray rotational modulation are exceptional among stars, one of the main reasons being that the X-ray brightest rapid rotators are highly active; such stars are probably covered with numerous active regions, and intense flaring may further veil low-amplitude modulations.

Among somewhat less active stars, the young solar analog EK Dra has shown rotational modulation both at X-ray and radio wavelengths (Güdel et al. 1995), and in X-rays it is predominantly the cooler material that shows this modulation. This argues against flares contributing to the signal. The depth and length of the modulation (Fig. 18a) constrains the X-ray coronal height, and also the electron densities to $n_{e}>4 \times 10^{10} \mathrm{~cm}^{-3}$, in agreement with spectroscopic measurements (Ness et al. 2004). This leads to the conclusion that much of the emitting material is concentrated in large "active regions". Collier Cameron et al. (1988) reported a similar finding for a weak modulation in AB Dor: this again suggested that the cooler loops are relatively compact. It is worthwhile mentioning, though, that X-ray rotational modulation has been difficult to identify on this star (S. White et al. 1996 and references therein; Vilhu et al. 1993; Maggio et al. 2000; Güdel et al. 2001b); a weak modulation has been reported by Kürster et al. (1997). Interestingly, radio observations of $\mathrm{AB}$ Dor reveal two emission peaks per rotation that probably relate to preferred active longitudes (Lim et al. 1992).
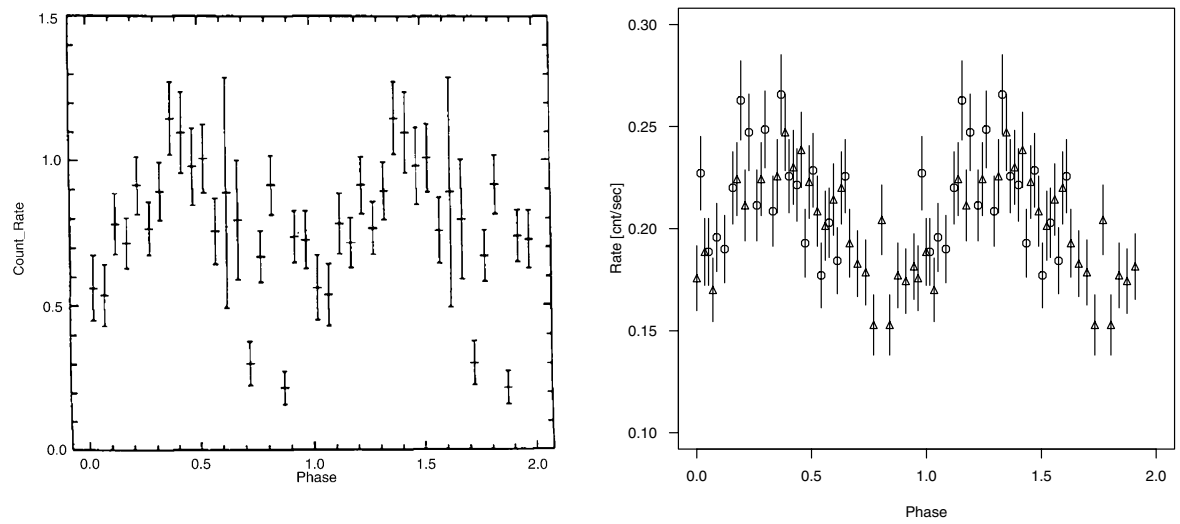

Fig. 18. Two examples of X-ray rotational modulation in active solar analogs: a (left): EK Dra (Güdel et al. 1995), and $b$ (right), the supersaturated VXR45 (right figure courtesy of A. Marino, after Marino et al. 2003a). Both light curves are phase-folded 
Because the X-ray luminosity in "supersaturated stars" (Sect. 5) is also below the empirical maximum, rotational modulation would give important structural information on the state of such coronae. A deep modulation in VXR45 (Fig. 18b) suggests that extreme activity in these stars is again not due to complete coverage of the surface with active regions (Marino et al. 2003a).

Among evolved stars, the RS CVn binary HR 1099 (K1 IV + G5 V) has consistently shown X-ray and EUV rotational modulation, with an X-ray maximum at phases when the larger K subgiant is in front (Agrawal and Vaidya 1988; J. Drake et al. 1994; Audard et al. 2001a). Because the X-ray material is almost entirely located on the $\mathrm{K}$ star (Ayres et al. 2001b), the rotationally modulated material can be located - in contrast to the binaries studied through eclipses - on the K star hemisphere that faces away from the companion (Audard et al. 2001a). A particularly clear example was presented by Ottmann (1994) for Algol that showed a closely repeating pattern over three stellar orbits, testifying to the stability of the underlying coronal structure on time scales of several days. The strong modulation combined with a large inclination angle further suggests that most of the modulated material is located at moderate latitudes. Schmitt et al. (1996d) and Gunn et al. (1997) found strong rotational modulation on the RS CVn-type binary CF Tuc. Here, Gunn et al. (1997) speculated that the emitting corona is associated with the larger K subgiant and is facing toward the smaller companion, thus again opening up a possibility for intrabinary magnetic fields.

Somewhat unexpectedly, even extremely active protostars appear to show signs of coronal inhomogeneities. Kamata et al. (1997) observed sinusoidal variations in one such object and tentatively interpreted it as the signature of rapid ( $P \approx 1 \mathrm{~d}$ ) rotation. If this interpretation is correct, then once again we infer that these extremely active stars are not fully covered by coronal active regions.

\subsection{Rotationally modulated and eclipsed X-ray flares}

Rotational modulation of flares, or the absence thereof, contributes very valuable information on densities and the geometric size of flaring structures. Skinner et al. (1997) found compelling evidence for a rotationally modulated flare on the T Tau star V773 Tau. By making use of (24), they inferred, independent of any flare model, a minimum electron density of $2 \times 10^{11} \mathrm{~cm}^{-3}$ in the flaring region. This immediately implies that the decaying plasma is subject to continuous heating. Otherwise, the plasma would freely cool on a time scale of $\approx 1.5 \mathrm{hrs}$, an order of magnitude shorter than observed. Geometric considerations then lead to a source of modest size at high latitudes. Stelzer et al. (1999) developed a "rotating flare" model that combines flare decay with self-eclipse of the flaring volume by the rotating star. They successfully fitted light curves of four large stellar flares with slow rises and flat peaks. Alternative explanations for such anomalous light curves are possible, however.

If a long-lasting flare with time scales exceeding one orbit period shows no eclipse in its course, then the flare either occurred near one of the polar regions, or it is geometrically large. Kürster and Schmitt (1996) argued in favor of the latter possibility; they modeled a flare light-curve of CF Tuc. Nevertheless, a partial eclipse may also have affected the decay of that flare. Maggio et al. (2000) suggested flaring loops located at latitudes higher than $60^{\circ}$ based on the absence of eclipse features during a large flare on AB Dor. 


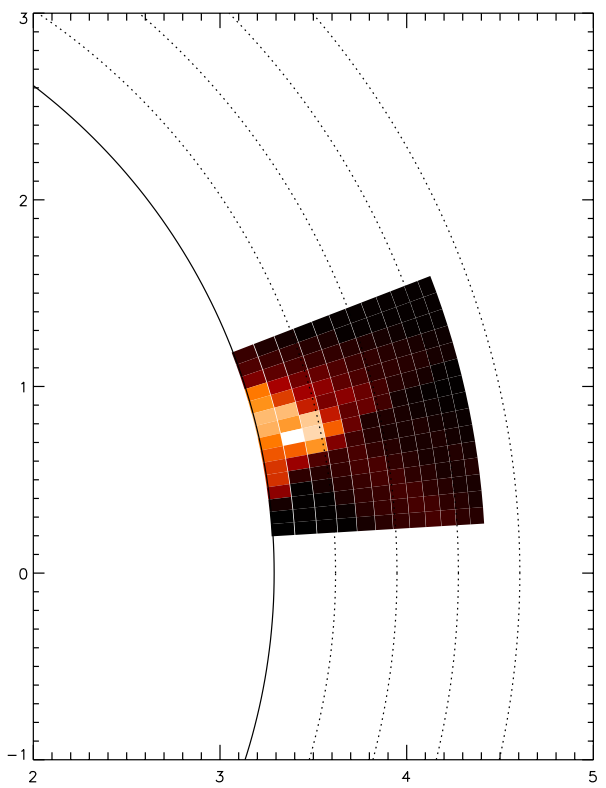

Fig. 19. Limb view of an X-ray flare on Algol, reconstructed from an eclipse light curve. Axis labels are in units of $R_{\odot}$. Dashed circles give height in steps of $0.1 R_{*}$ (figure courtesy of J.-U. Ness, after Schmitt et al. 2003)

This would allow for more modest flare sizes, a possibility that is clearly supported by detailed flare modeling results (Sect. 12).

Choi and Dotani (1998) were the first to describe a full eclipse of an X-ray flare in progress in a binary system, namely in the contact binary system VW Cep. During a narrow dip in the flare decay, the X-ray flux returned essentially to the pre-flare level. Geometric considerations then placed the flare near one of the poles of the primary star, with a size scale of order $5.5 \times 10^{10} \mathrm{~cm}$ or somewhat smaller than the secondary star. The authors consequently inferred an electron density of $5 \times 10^{10} \mathrm{~cm}^{-3}$. A polar location was also advocated for a flare on Algol observed across an eclipse by Schmitt and Favata (1999). The flare emission was again eclipsed completely, and judged from the known system geometry, the flare was located above one of the poles, with a maximum source height of no more than approximately $0.5 R_{*}$, implying a minimum electron density of $9.4 \times 10^{10} \mathrm{~cm}^{-3}$ if the volume filling factor was unity. A more moderate flare was observed during an eclipse in the Algol system by Schmitt et al. (2003) (Fig. 19). In this case, the image reconstruction required an equatorial location, with a compact flare source of height $h \approx 0.1 R_{*}$. Most of the source volume exceeded densities of $10^{11} \mathrm{~cm}^{-3}$, with the highest values at $\approx 2 \times 10^{11} \mathrm{~cm}^{-3}$. Because the quiescent flux level was attained throughout the flare eclipse, the authors argued that its source, in turn, must be concentrated near the polar region with a modest filling factor of $f<0.1$ and electron densities of $\approx 3 \times 10^{10} \mathrm{~cm}^{-3}$.

Further candidates for eclipsed flares may be found in Bedford et al. (1990) for the short-period binary XY UMa as suggested by Jeffries (1998), and in Güdel et al. (2001a) for the eclipsing M dwarf binary YY Gem. Briggs and Pye (2003) reported on 


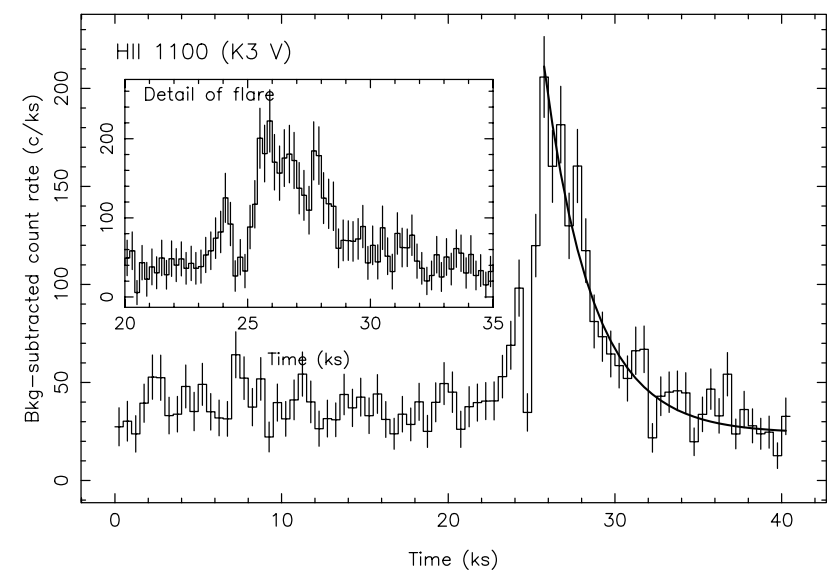

Fig. 20. A flare on the Pleiades member HII 1100 that shows a short dip early in the rise time, with the flux dropping back to the pre-flare level. One hypothesis (that requires confirmation) refers to an eclipsing Jupiter-like planet (figure courtesy of K. Briggs, after Briggs and Pye 2003, based on XMM-Newton observations)

an interesting eclipse-like feature in a flare in progress on an active Pleiades member (Fig. 20). Again, the flux returned precisely to pre-flare values for a short time, only to resume the increase of the flare light-curve after a brief interval. A somewhat speculative but quite possible cause for the observed dip could be a planet in transit. The existence of such a planet obviously needs confirmation. Some important parameters related to the flares discussed in this section are summarized in Table 3.

\subsection{Inferences from Doppler measurements}

Doppler information from X-ray spectral lines may open up new ways of imaging stellar coronae as they rotate, or as they orbit around the center of gravity in binaries. First attempts are encouraging although the instrumental limitations are still severe. Ayres et al. (2001b) found Doppler shifts with amplitudes of $\approx 50 \mathrm{~km} \mathrm{~s}^{-1}$ in X-ray lines of HR 1099 (Fig. 21). Amplitudes and phases clearly agree with the line-of-sight orbital velocity of the subgiant $\mathrm{K}$ star, thus locating the bulk of the X-ray emitting plasma on this star, rather than in the intrabinary region. Periodic line broadening in YY Gem, on the other hand, suggests that both components are similarly X-ray luminous (Güdel et al. 2001a); this is expected, because this binary consists of two almost identical $\mathrm{M}$ dwarfs. Huenemoerder et al. (2003) found Doppler motions in AR Lac to be compatible with coronae on both companions if the plasma is close to the photospheric level. For the contact binary $44 \mathrm{i}$ Boo, Brickhouse et al. (2001) reported periodic line shifts corresponding to a total net velocity change over the full orbit of $180 \mathrm{~km} \mathrm{~s}^{-1}$. From the amplitudes and the phase of the rotational modulation (Brickhouse and Dupree 1998), they concluded that two dominant X-ray sources were present, one being very compact and the other being extended, but both being located close to the stellar pole of the larger companion.

A rather new technique employs coronal forbidden lines in the UV or optical range, making use of spectral resolving powers that are still out of reach to $\mathrm{X}$-ray astronomy. 


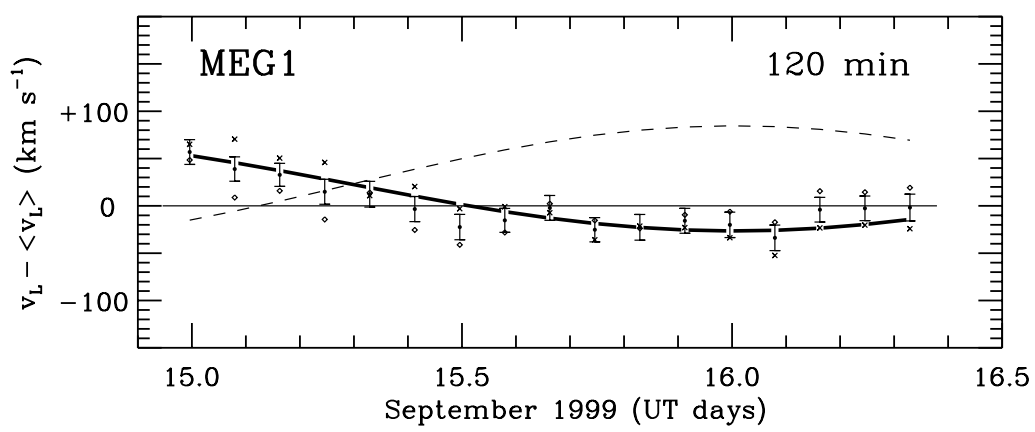

Fig. 21. Doppler motion in the HR 1099 system measured from line shifts in the Chandra HETGS/MEG spectrum. The predicted radial velocity curves are shown solid (K star) and dashed ( $\mathrm{G}$ star). The measurements clearly locate the X-ray emission predominantly on the $\mathrm{K}$ star (figure courtesy of T. Ayres, after Ayres et al. 2001b)

Maran et al. (1994) and Robinson et al. (1996) were the first to report the detection of the Fe XXI $\lambda 1354$ line in $H S T$ observations of active stars. Linsky et al. (1998) presented a detailed analysis of this line in the Capella system. Ayres et al. (2003b) summarized the current status and presented a survey of further possible UV line candidates. In the UV range, the second promising candidate is the Fe XII $\lambda 1349$ line while few other transitions are sufficiently strong for detection. An analogous study for the far-UV range was presented by Redfield et al. (2003). In the UV range accessible from the ground, Schmitt and Wichmann (2001) for the first time recorded the Fe XIII $\lambda 3388.1$ line formed at 1.6 MK in a spectrum of the dMe dwarf CN Leo.

The collected results from these observations are still modest - compared to the X-ray bibliography! Tentative results seem promising for further investigation:

1. the lines observed so far are essentially only thermally and rotationally broadened, i.e., significant bulk Doppler shifts due to mass flows in flares have not (yet) been detected;

2. several very active stars appear to show some excess broadening; it could possibly be due to rotational velocities of extended coronal regions located high above the stellar surface. Line broadening may in this case provide some important information on the overall coronal size (Ayres et al. 2003b; Redfield et al. 2003).

\subsection{Inferences from surface magnetic fields}

Information on coronal structure can also be derived indirectly from surface ZeemanDoppler images as developed for and applied to the stellar case by Jardine et al. (2002a), Jardine et al. (2002b), and Hussain et al. (2002) and further references therein. Because Zeeman-Doppler images provide the radial and azimuthal magnetic-field strengths as a function of position, one could in principle derive the coronal magnetic field structure by 3-D extrapolation. This requires a number of assumptions, however. Jardine et al. (2002a) and Jardine et al. (2002b) studied the case of potential field extrapolation, i.e., the coronal magnetic field follows $\mathbf{B}=-\nabla \Psi$, where $\Psi$ is a function of the coordinates. Because the field must be divergence free, one requires $\nabla^{2} \Psi=0$. The solution involves 


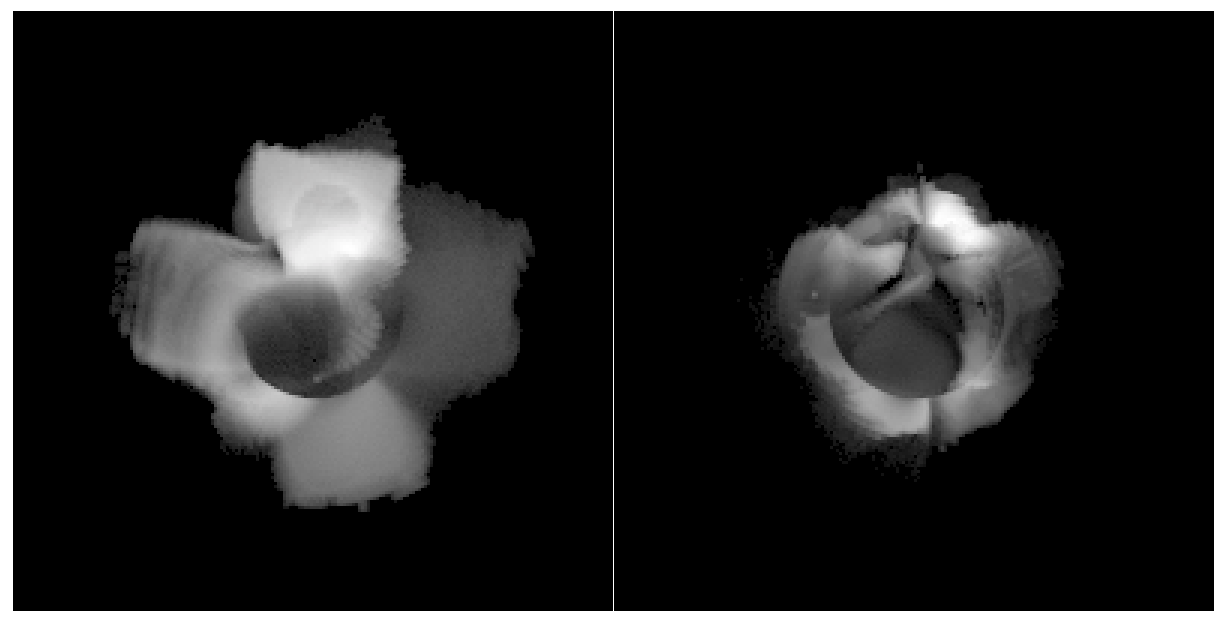

Fig. 22. Reconstruction of the X-ray corona of AB Dor based on extrapolation from Zeeman Doppler Imaging. Brightness encodes emission measure along the line of sight. The left panel shows a solution for an EM-weighted density of $4 \times 10^{8} \mathrm{~cm}^{-3}$, the right panel for $1.5 \times 10^{10} \mathrm{~cm}^{-3}$ (figure courtesy of M. Jardine, after Jardine et al. 2002b)

associated Legendre functions in spherical coordinates, and the boundary conditions, namely the measured magnetic field strengths on the surface, fix the coefficients.

The model requires further parameters such as the base thermal pressure with respect to the local magnetic pressure, and some cutoff of the corona at locations where the thermal pressure might open up the coronal field lines. Various computed models (Fig. 22) recover, at least qualitatively, the total EM, the average density, and the low level of rotational modulation observed on very active stars such as AB Dor. The highly complex coronal structure, involving both very large magnetic features and more compact loops anchored predominantly at polar latitudes (as implied by the Doppler images), suppresses X-ray rotational modulation to a large extent.

The modeling is delicate because i) part of the star cannot usually be Doppler imaged, ii) fine structures are not recognized in the available Doppler images, in particular so in dark areas, and iii) the active corona is not in a potential configuration. The first point was addressed by Jardine et al. (2002a) and Jardine et al. (2002b) and the last by Hussain et al. (2002) who extended the models to include some form of currents in force-free fields. It is interesting that the models show various locations where the gravity, centrifugal, and Lorentz forces are in equilibrium. These are the places where distant $\mathrm{H} \alpha$ prominences may condense, for which there is indeed evidence in $\mathrm{AB}$ Dor out to distances of $5 R_{*}$ (Collier Cameron 1988; Donati 1999).

\subsection{Extended or compact coronae?}

As the previous discussions imply, we are confronted with mixed evidence for predominantly extended (source height $>R_{*}$ ) and predominantly compact ( $\ll R_{*}$ ) coronal structures or a mixture thereof. There does not seem to be unequivocal agreement on the 
type of structure that generally prevails. Several trends can be recognized, however, as summarized below.

Compact coronal structure. Steep (portions of the) ingress and egress light curves or prominent rotational modulation unambiguously argue in favor of short scale lengths perpendicular to the line of sight (e.g., for the G star in AR Lac, see Walter et al. 1983; White et al. 1990; Ottmann et al. 1993; or for $\alpha$ CrB, see Schmitt and Kürster 1993; Güdel et al. 2003b). Common to all are relatively high inferred densities $\left(\approx 10^{10} \mathrm{~cm}^{-3}\right)$. The pressures of such active regions may exceed pressures of non-flaring solar active regions by up to two orders of magnitude. Spectroscopic observations of high densities and loop modeling add further evidence for the presence of some rather compact sources (Sects. 10 and 11.2). Flare modeling also provides modest sizes, often of order $0.1-1 R_{*}$, for the involved magnetic loops (Sect. 12).

Extended structure. Here, the arguments are less direct and are usually based on the absence of deep eclipses, or very shallow ingress and egress curves (e.g., the K star in AR Lac, see Walter et al. 1983; White et al. 1990). Caution is in order in cases where the sources may be located near one of the polar regions; in those cases, eclipses and rotational modulation may also be absent regardless of the source size. Complementary information is available from flare analysis (see Sect. 12) that in some cases does suggest quite large loops. The caveat here is that simple single-loop models may not apply to such flares. Clear evidence is available from radio interferometry that proves the presence of large-scale, globally ordered magnetic fields (see references in Güdel 2002). The existence of prominent extended, closed magnetic fields on scales $>R_{*}$ is therefore also beyond doubt for several active stars.

The most likely answer to the question on coronal structure size is therefore an equivocal one: Coronal magnetic structures follow a size distribution from very compact to extended ( $z R_{*}$ ) with various characteristic densities, temperatures, non-thermal electron densities, and surface locations. This is no different from what we see on the Sun even though various features observed on stars stretch the comparison perhaps rather too far for comfort: various structures may predominate, depending on the magnetic activity level, on the depth of the convection zone, or on binary characteristics.

\section{Stellar X-ray flares}

Flares arise as a consequence of a sudden energy release and relaxation process of the magnetic field in solar and stellar coronae. Present-day models assume that the energy is accumulated and stored in non-potential magnetic fields prior to an instability that most likely implies reconnection of neighboring antiparallel magnetic fields. The energy is brought into the corona by turbulent footpoint motions that tangle the field lines at larger heights. The explosive energy release becomes measurable across the electromagnetic spectrum and, in the solar case, as high-energy particles in interplanetary space as well. For a review, I refer the reader to Haisch et al. (1991a).

Flares are ubiquitous among coronal stars, with very few exceptions. Apart from the entire main sequence (Schmitt 1994), flares have been found among giants (Welty and Ramsey 1994 for FK Com) and hybrid stars (Kashyap et al. 1994; Hünsch and Reimers 1995), and in clump giants both pre-He-flash (Ayres et al. 1999) and post-He-flash (Ayres 
et al. 2001a, Fig. 25 below), partly with extremely long time-scales of up to several days and with signs of continued activity.

Flares have prominently figured in solar studies, and it is once again solar physics that has paved the way to the interpretation of stellar flares, even if not all features are fully understood yet. The complexity that flares reveal to the solar astronomer is inaccessible in stellar flares, especially in the absence of spatially resolved observations. Simplified concepts, perhaps tested for solar examples, must suffice. The following sections summarize the "stellar astronomer's way" of looking at flares.

\subsection{General properties and classifications}

X-ray flares on the Sun come, roughly speaking, in two varieties, known as compact and long-duration (also "two-ribbon") flares (Pallavicini et al. 1977). The former variant shows a simple structure, usually consisting of one or a few individual loops that brighten up on time scales of minutes. They are of modest height and show high densities. The most likely mechanism leading to compact flares is an interaction between neighboring loops. In contrast, the second type of flare shows decay time scales of up to 1-2 hours; their magnetic field structures are large $\left(10^{4}-10^{5} \mathrm{~km}\right)$ and the densities are low. Complex loop arcades that are anchored in two $\mathrm{H} \alpha$ ribbons are regularly involved. The most likely flare process relates to an opening up of magnetic fields (e.g., by a filament eruption) and subsequent relaxation by closing the "open" field lines. Related flare classifications have been made for hard X-rays as well.

Applications of the solar classification scheme to stars, in particular magnetically active stars, should be treated with caution. The possibility of intrabinary magnetic fields or significant tidal effects in close binary stars, extremely dense packing of magnetic fields, polar magnetic fields, magnetic fields from a distributed dynamo, magnetospheres of global dimensions, and star-disk magnetic fields in pre-main sequence stars may lead to energy release configurations that are unknown on the Sun. The comprehensive EXOSAT survey of MS stellar flares by Pallavicini et al. (1990a) testifies to this problem, with some flares showing more rapid decays than rises, multiple peaks, abrupt drops etc.

Nevertheless, these authors did find evidence for a class of flares with short rise times of order of minutes and decay times of order of a few tens of minutes, proposed to be analogs of solar compact flares, and flares with long decay times exceeding one hour, reminiscent of two-ribbon (2-R) flares on the Sun. Flares with a rapid rise on time scales smaller than the dynamical time scale of filament eruption are unlikely to be 2-R flares but are more suggestive of compact flares (van den Oord et al. 1988), while longer rise times may indicate 2-R flares (e.g., van den Oord and Mewe 1989). Typical e-folding decay times of large flares on active stars are found to be several kiloseconds (Gotthelf et al. 1994; Monsignori Fossi et al. 1996; Sciortino et al. 1999; Maggio et al. 2000). Decay times of 5-15 ks are not exceptional for young stars, e.g., in the Pleiades or in star-forming regions (Gagné et al. 1995a; Stelzer et al. 2000). In any case, the natural approach to understanding stellar coronal X-ray flares has been to extend solar concepts to stellar environments. I will now first discuss several flare models that have been repeatedly applied to observations in the literature; the subsequent sections will summarize a few notable results derived from stellar flare observations. 


\subsection{General flare scenario}

A "standard picture" has emerged from numerous solar flare studies, comprising roughly the following features. The flare reconnection region, located somewhere at large coronal heights, primarily accelerates electrons (and possibly ions) up to $\mathrm{MeV}$ energies. The accelerated electrons precipitate along the magnetic fields into the chromosphere where they heat the cool plasma to coronal flare temperatures, thus "evaporating" part of the chromosphere into the corona. The high-energy electron population emits radio gyrosynchrotron radiation and, upon impact, non-thermal hard X-ray (HXR) bremsstrahlung, and it generates optical continuum+line radiation. These emissions are well correlated on time scales as short as seconds (e.g., Hudson et al. 1992 for HXR and white light flares; Kosugi et al. 1988 for hard X-rays and gyrosynchrotron emission). The soft Xrays, in contrast, develop only as the closed magnetic fields are filled with plasma on time scales of tens of seconds to minutes. Various elements of this scenario may vary from flare to flare.

From the X-ray point of view, the above model implies a characteristic evolution of flare EM and temperature. As the initial energy release suddenly heats part of the chromospheric plasma, very high temperatures are reached rapidly. As large amounts of plasma are streaming into the corona, cooling starts while the luminosity is still increasing as a consequence of increasing densities. The flare temperature thus peaks before the EM does, or analogously, harder emission peaks before softer emission, a feature that is regularly observed in solar and stellar flares ${ }^{4}$ (Landini et al. 1986; Haisch et al. 1987; Vilhu et al. 1988; Doyle et al. 1988a; van den Oord et al. 1988; van den Oord and Mewe 1989; Haisch et al. 1990b; Stern et al. 1992a; Monsignori Fossi et al. 1996; Güdel et al. 1999; Maggio et al. 2000; Güdel et al. 2004) and in numerical simulations of flares (e.g., Cheng and Pallavicini 1991; Reale et al. 2004).

\subsection{Cooling physics}

Flares cool through radiative, conductive, and possibly also volume expansion processes. We define the flare decay phase as the episode when the net energy loss by cooling exceeds the energy gain by heating, and the total thermal energy of the flare plasma decreases. The thermal energy decay time scale $\tau_{\text {th }}$ is defined as

$$
\tau_{\mathrm{th}}=\frac{E}{\dot{E}}
$$

where $E \approx 3 n_{e} k T$ is the total thermal energy density in the flaring plasma of electron density $n_{e}$ and temperature $T$, and $\dot{E}$ is the volumetric cooling loss rate (in erg $\mathrm{cm}^{-3} \mathrm{~s}^{-1}$ ). For conduction across temperature gradients in parallel magnetic fields, the mean loss rate per unit volume is

$$
\dot{E}_{c}=\frac{1}{L} \kappa_{0} T^{5 / 2} \frac{d T}{d s} \approx \frac{4}{7 L^{2}} \kappa_{0} T^{7 / 2}
$$

\footnotetext{
${ }^{4}$ This should not be mistaken for the Neupert Effect (Sect. 12.16); the present effect is entirely due to plasma cooling while the Neupert effect involves a physically different non-thermal population of electrons. The different hard and soft light-curves do not per se require non-thermal plasma, nor multiple components.
} 
where $s$ is the coordinate along the field lines, and the term $\kappa_{0} T^{5 / 2} d T / d s$ is the conductive flux in the approximation of Spitzer (1962), to be evaluated near the loop footpoint where $T$ drops below $10^{6} \mathrm{~K}$, with $\kappa_{0} \approx 9 \times 10^{-7} \mathrm{erg} \mathrm{cm}^{-1} \mathrm{~s}^{-1} \mathrm{~K}^{-7 / 2}$. Equations (31, 32 ) define the conductive time scale $\tau_{\text {th }} \equiv \tau_{c}$. The second equation in (32) should be used only as an approximation for non-radiating loops with a constant cross section down to the loss region and with uniform heating (or for time-dependent cooling of a constant-pressure loop without heating; for the factor of 4/7, see Dowdy et al. 1985; Kopp and Poletto 1993). We have used $L$ for the characteristic dimension of the source along the magnetic field lines, for example the half-length of a magnetic loop. Strictly speaking, energy is not lost by conduction but is redistributed within the source; however, we consider energy lost when it is conducted to a region that is below X-ray emitting temperatures, e.g., the transition region/chromosphere at the magnetic loop footpoints. For expressions relevant for loops with varying cross sections, see van den Oord and Mewe (1989).

Radiative losses are by bremsstrahlung (dominant for $T \gtrsim 20 \mathrm{MK}$ ), 2-photon continuum, bound-free, and line radiation. We note that the plasma composition in terms of element abundances can modify the cooling function $\Lambda(T)$, but the correction is of minor importance because stellar flares are usually rather hot. At relevant temperatures, the dominant radiative losses are by bremsstrahlung, which is little sensitive to modifications of the heavy-element abundances. The energy loss rate is

$$
\dot{E}_{r}=n_{e} n_{H} \Lambda(T)
$$

(or $n_{e}^{2} \Lambda^{\prime}(T)$ in an alternative definition, with $n_{\mathrm{H}} \approx 0.85 n_{e}$ for cosmic abundances). For $T \geq 20 \mathrm{MK}, \Lambda(T)=\Lambda_{0} T^{\gamma} \approx 10^{-24.66} T^{1 / 4} \mathrm{erg} \mathrm{cm}^{3} \mathrm{~s}^{-1}$ (after van den Oord and Mewe 1989 and Mewe et al. 1985). Equations. $(31,33)$ define the radiative time scale $\tau_{\text {th }} \equiv \tau_{r}$.

\subsection{Interpretation of the decay time}

Equations (31), (32), and (33) describe the decay of the thermal energy, which in flare plasma is primarily due to the decay of temperature (with a time scale $\tau_{T}$ ) and density. In contrast, the observed light curve decays (with a time scale $\tau_{d}$ for the luminosity) primarily due to the decreasing EM and, to a lesser extent, due to the decrease of $\Lambda(T)$ with decreasing temperature above $\approx 15 \mathrm{MK}$. From the energy equation, the thermal energy decay time scale $\tau_{\text {th }}$ is found to be

$$
\frac{1}{\tau_{\mathrm{th}}}=\left(1-\frac{\gamma}{2}\right) \frac{1}{\tau_{T}}+\frac{1}{2 \tau_{d}}
$$

where the right-hand side is usually known from the observations (see van den Oord et al. 1988 for a derivation). The decay time scale of the EM then follows as $1 / \tau_{\mathrm{EM}}=$ $1 / \tau_{d}-\gamma / \tau_{T}$. Pan et al. (1997) derived somewhat different coefficients in (34) for the assumption of constant volume or constant mass, including the enthalpy flux. In the absence of measurements of $\tau_{T}$, it is often assumed that $\tau_{\mathrm{th}}=\tau_{d}$ although this is an inaccurate approximation. For a freely cooling loop, $\tau_{\mathrm{EM}}=\tau_{T}$ (Sect. 12.6), and a better replacement is therefore $\tau_{\text {th }}=2(\gamma+1) \tau_{d} / 3$. 
In (34), $\tau_{\text {th }}$ is usually set to be $\tau_{r}$ or $\tau_{c}$ or, if both loss terms are significant, $\left(\tau_{r}^{-1}+\right.$ $\left.\tau_{c}^{-1}\right)^{-1}$, taken at the beginning of the flare decay (note again that a simple identification of $\tau_{r}$ with $\tau_{d}$ is not accurate). If radiative losses dominate, the density immediately follows from Eqs. $(31,33)$

$$
\tau_{\mathrm{th}} \approx \frac{3 k T}{n_{e} \Lambda(T)}
$$

and the characteristic size scale $\ell$ of the flaring plasma or the flare-loop semi-length $L$ for a sample of $N$ identical loops follow from

$$
\mathrm{EM}=n_{e} n_{H}(\Gamma+1) \pi \alpha^{2} N L^{3} \approx n^{2} \ell^{3}
$$

where $\alpha$ is the aspect ratio (ratio between loop cross sectional diameter at the base and total length $2 L$ ) and $\Gamma$ is the loop expansion factor. The loop height for the important case of dominant radiative losses follows to be (White et al. 1986; van den Oord et al. 1988)

$$
H=\left(\frac{8}{9 \pi^{4}} \frac{\Lambda_{0}^{2}}{k^{2}}\right)^{1 / 3}\left(\frac{\mathrm{EM}}{T^{3 / 2}} \tau_{r}^{2}\right)^{1 / 3}\left(N \alpha^{2}\right)^{-1 / 3}(\Gamma+1)^{-1 / 3} .
$$

A lower limit to $H$ is found for $\tau_{r} \approx \tau_{c}$ in the same treatment:

$$
H_{\min }=\frac{\Lambda_{0}}{\kappa_{0} \pi^{2}} \frac{\mathrm{EM}}{T^{3.25}}\left(N \alpha^{2}\right)^{-1} .
$$

$N, \alpha$, and $\Gamma$ are usually unknown and treated as free parameters within reasonable bounds. Generally, a small $N$ is compatible with dominant radiative cooling.

This approach gives a first estimate for the flare-loop size, but it provides only an upper limit to $\ell$ and a lower limit to $n_{e}$, for the following reason. Equation (35) assumes free cooling without a heating contribution. If heating continues during the decay phase, then $\tau_{\mathrm{obs}}>\tau_{\mathrm{th}}$, hence the implied $n_{e, \text { obs }}<n_{e}$; in other words, the effective cooling function $\Lambda$ is reduced and, therefore, the apparent $\ell_{\text {obs }}>\ell$. The effect of continued heating will be discussed in Sect. 12.6 and 12.7.

Decay time methods have been extensively used by, among others, Haisch et al. (1980), van den Oord et al. (1988), Jeffries and Bedford (1990), Doyle et al. (1991), Doyle et al. (1992b), Ottmann and Schmitt (1994), Mewe et al. (1997), and Osten et al. (2000) for the interpretation of large flares. For small $N$, most authors found loop heights of the order of a few $10^{10} \mathrm{~cm}$ and inferred densities of a few times $10^{11} \mathrm{~cm}^{-3}$ (see Sect. 12.11). Pallavicini et al. (1990a) inferred typical flare densities, volumes, and magnetic loop lengths for various strong flares on $\mathrm{M}$ dwarfs, concluding that $n_{e}$ tends to be higher than electron densities in solar compact flares, while the volumes are more reminiscent of solar two-ribbon (2-R) flares.

The simplest approach involving full coronal loop models assumes cooling that is completely governed either by conduction or radiation. Antiochos and Sturrock (1976, 1978) have treated a conductively-driven flaring loop, first with static and then with evaporative cooling, i.e., respectively, without mass flow and with subsonic mass upflows (under time-constant pressure). Under the assumption that radiation is negligible, Antiochos and Sturrock obtained as loop-apex temperatures

$$
T_{\text {stat }}(t)=T_{0}\left(1+\frac{t}{\tau_{c, 0}}\right)^{-2 / 5}, \quad T_{\text {evap }}(t)=T_{0}\left(1+\frac{t}{\tau_{c, 0}}\right)^{-2 / 7} .
$$


Here, the relevant timescale is $\tau_{c, 0}=5 p_{0} /\left(2 \kappa_{0} T_{0}^{7 / 2}\right)(L / 1.6)^{2}$ (Cargill 1994), and the subscript 0 refers to values at the beginning of the cooling phase. This formulation has been used by Pan et al. (1997) as part of an extended flare model involving variable mass or variable volume. For the more likely case of dominant radiative cooling, Antiochos (1980) and Cargill (1994) give, for static cooling and cooling with subsonic draining, respectively, a temperature at the position $s$ along the loop of

$$
T(s, t)= \begin{cases}T_{0}(s)\left[1-(1-\gamma) \frac{t}{\tau_{r, 0}}\right]^{1 /(1-\gamma)} & \text { static } \\ T_{0}(s)\left[1-\frac{3}{2}\left(\frac{1}{2}-\gamma\right) \frac{t}{\tau_{r, 0}}\right]^{1 /(1 / 2-\gamma)} & \text { draining }\end{cases}
$$

where $\tau_{r, 0}$ is the radiative loss time (35) at the cooling onset.

A more general treatment was considered by Pan and Jordan (1995) for a flare on the active main-sequence binary CC Eri, including both radiative and conductive losses. They gave a differential equation for the development of the temperature as a function of the measured EM and $T$, which then serves to determine $L$ in the conductively-dominated case, and the flaring volume in the radiative case.

\subsection{Quasi-static cooling loops}

van den Oord and Mewe (1989) derived the energy equation of a cooling magnetic loop in such a way that it is formally identical to a static loop (Rosner et al. 1978), by introducing a slowly varying flare heating rate that balances the total energy loss, and a possible constant heating rate during the flare decay. This specific solution thus proceeds through a sequence of different (quasi-)static loops with decreasing temperature.

The general treatment involves continued heating that keeps the cooling loop at coronal temperatures. If this constant heating term is zero, one finds for free quasi-static cooling

$$
\begin{aligned}
T(t) & =T_{0}\left(1+t / 3 \tau_{r, 0}\right)^{-8 / 7} \\
L_{r}(t) & =L_{r, 0}\left(1+t / 3 \tau_{r, 0}\right)^{-4} \\
n_{e}(t) & =n_{e, 0}\left(1+t / 3 \tau_{r, 0}\right)^{-13 / 7}
\end{aligned}
$$

where $L_{r}$ is the total radiative loss rate, and $\tau_{r, 0}$ is the radiative loss time scale (35) at the beginning of the flare decay.

This prescription is equivalent to requiring a constant ratio between radiative and conductive loss times, i.e., in the approximation of $T \gtrsim 20 \mathrm{MK}\left(\Lambda \propto T^{1 / 4}\right)$

$$
\frac{\tau_{r}}{\tau_{c}}=\mathrm{const} \frac{T^{13 / 4}}{\mathrm{EM}} \approx 0.18 .
$$

Accordingly, the applicability of the quasi-static cooling approach can be supported or rejected based on the run of $T$ and EM during the decay phase. Note, however, that a constant ratio (44) is not a sufficient condition to fully justify this approach.

The quasi-static cooling model also predicts a particular shape of the flare DEM (Mewe et al. 1997) - see (12). van den Oord and Mewe (1989), Stern et al. (1992a), 
Ottmann and Schmitt (1996), Kürster and Schmitt (1996), Mewe et al. (1997) and many others have applied this model. The approach has recently been criticized, however, because the treatment of heating is not physically self-consistent (Reale 2002).

\subsection{Cooling loops with continued heating}

The characteristics of the flare decay itself strongly depend on the amount of ongoing heating. Several models include continued heating with some prescription (e.g., the quasi-static cooling approach, or two-ribbon models). Continued heating in several large flares with a rapid rise has been questioned for cases where the thermal plasma energy content at flare peak was found to be approximately equal to the total radiative energy during the complete flare. If that is the case, the flare energy has been deposited essentially before the flare peak (van den Oord et al. 1988; Jeffries and Bedford 1990; Tagliaferri et al. 1991). Other flares, however, exhibit evidence of reheating or continued heating during the decay phase (e.g., Tsuboi et al. 1998).

Whether or not flaring loops indeed follow a quasi-static cooling path is best studied on a density-temperature diagram (Fig. 23). Usually, characteristic values $T=T_{\mathrm{a}}$ and $n_{e}=n_{e, \text { a }}$ as measured at the loop apex are used as diagnostics. For a magnetic loop in hydrostatic equilibrium, with constant cross section assumed, the RTV scaling law (20) requires stable solutions $\left(T, n_{e}\right)$ to be located where $T^{2} \approx 7.6 \times 10^{-7} n_{e} L$ (for $n_{e}=n_{i}$ ). On a diagram of $\log T$ vs. $\log n_{e}$, all solutions are therefore located on a straight line with slope $\zeta=0.5$. Jakimiec et al. (1992) studied the paths of hydrodynamically simulated flares with different heating histories. The initial rapid heating leads to a rapid increase of $T$, inducing increased losses by conduction. As chromospheric evaporation grows,

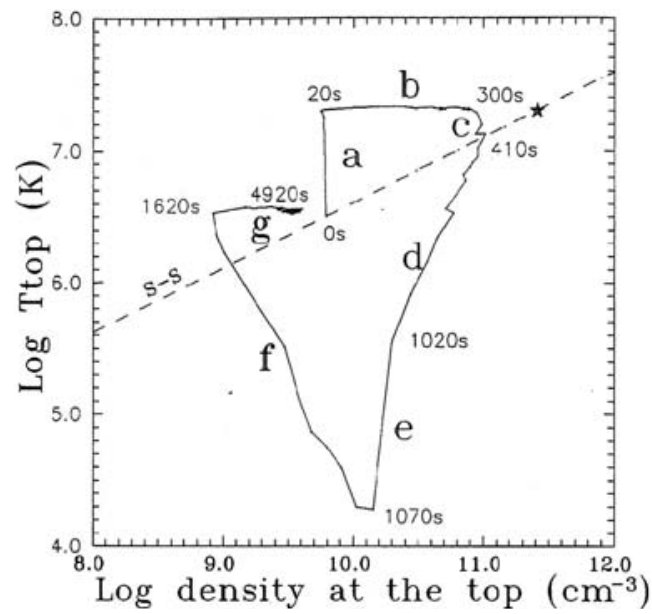

Fig. 23. Density-temperature diagram of a hydrodynamically simulated flare. The flare loop starts from an equilibrium (S-S, steady-state loop according to Rosner et al. 1978); (a) and (b) refer to the heating phase; at (c), the heating is abruptly turned off, after which the loop cools rapidly (d, e), and only slowly recovers toward a new equilibrium solution (f, g) due to constant background heating (from Jakimiec et al. 1992) 
radiation helps to balance the heating energy input. The flare decay sets in once the heating rate drops. At this moment, depending on the amount of ongoing heating, the magnetic loop is too dense to be in equilibrium, and the radiative losses exceed the heating rate, resulting in a thermal instability. In the limit of no heating during the decay, that is, an abrupt turn-off of the heating at the flare peak, the slope of the path becomes

$$
\zeta \equiv \frac{d \ln T}{d \ln n_{e}} \equiv \frac{\tau_{n}}{\tau_{T}}=2
$$

implying $T(t) \propto n_{e}^{\zeta}(t)=n_{e}^{2}(t)$ (see Serio et al. 1991 for further discussion). Here, $\tau_{T}$ and $\tau_{n}$ are the e-folding decay times of the temperature and the electron density, respectively, under the assumption of exponential decay laws. Only for a non-vanishing heating rate does the loop slowly recover and eventually settle on a new equilibrium locus (Fig. 23). In contrast, if heating continues and is very gradually reduced, the loop decays along the static solutions $(\zeta=0.5)$. Observationally, this path is often followed by large solar flares (Jakimiec et al. 1992). Clearly, a comprehensive description of stellar flares should thus consider continued heating throughout the flare. However, the observables typically available to the stellar astronomer are only the run of the X-ray luminosity (hence the EM) and of the characteristic temperature $T$. The volume and the density $n_{e}$ are unknown and need to be estimated from other parameters.

To this end, Reale et al. (1997) replaced $n_{e}$ by the observable $\sqrt{\mathrm{EM}}$ and thus assumed a constant flare volume, and further introduced the following generalization. In the freely cooling case after an abrupt heating turnoff, the entropy per particle at the loop apex decays on the thermodynamic decay time

$$
\tau_{\mathrm{td}}=3.7 \times 10^{-4} \frac{L}{T_{0}^{1 / 2}} \quad[\mathrm{~s}]
$$

where $T_{0}$ is the flare temperature at the beginning of the decay (Serio et al. 1991; see Reale et al. 1993 for an extension to loops with $L$ up to a pressure scale height). For the general case with continued heating, Reale et al. (1997) wrote

$$
\tau_{\mathrm{LC}}=3.7 \times 10^{-4} \frac{L}{T_{0}^{1 / 2}} F(\zeta) \quad[\mathrm{s}]
$$

where $F(\zeta) \equiv \tau_{\mathrm{LC}} / \tau_{\mathrm{td}}$ is a correction function depending on the heating decay time via $\zeta$, and $\tau_{\mathrm{LC}}$ is the observed X-ray light curve decay timescale. $F(\zeta)$ is therefore to be numerically calibrated for each X-ray telescope.

The important point is that $F(\zeta)$ has been empirically found from solar observations and numerical simulations to be a hyperbolic or exponential function with three parameters that can be determined for a given instrument. With known $F$, (47) can be solved for $L$. This scheme thus offers i) an indirect method to study flaring loop geometries $(L)$, ii) a way of determining the rate and decay time scale of continued heating via $F(\zeta)$ and $\tau_{\mathrm{td}}$, and iii) implications for the density decay time via $\tau_{n}=\zeta \tau_{T}$. Conditions of applicability include $\zeta \geq 0.3$ and a resulting loop length $L$ of less than one pressure scale height (Reale et al. 1997).

Loop sizes derived from this method agree with direct observations on the Sun. Solar observations of moderate flares with the Solar Maximum Mission SMM implied 
$\zeta$ between $\sim 0.5$ and 2 (Sylwester et al. 1993), although Reale et al. (1997) found a predominance of values around $\sim 0.3-0.7$, i.e., flares with substantial sustained heating.

This method has been applied extensively in the interpretation of stellar flares. Modeling of large flares yields reasonable flare loop sizes that are often smaller than those inferred from other methods. Loop sizes comparable with sizes of solar active regions have been found. But such loops may also be of order of one stellar radius for M dwarfs such as Proxima Centauri (Reale et al. 1988, 2004). The flaring region may thus comprise a significant fraction of the stellar corona on such stars. Giant flares with reliable measurements of temperatures are particularly well suited for an application of this method. Examples have been presented for EV Lac (a flare with a $>100$ fold increase in count rate in the $A S C A$ detectors, reaching temperatures of $70 \mathrm{MK}$; Favata et al. 2000a, Fig. 25), AB Dor (Maggio et al. 2000), and Proxima Centauri (Reale et al. 2004). The same approach was also used for pre-main sequence stars by Favata et al. (2001), including comparisons with other methods.

The moderate loop sizes resulting from this method have important implications for coronal structure (see Sect.11). The magnetic loops related to several flares observed on AD Leo, for example, all seem to be of fairly similar, modest size (half-length $L \approx$ $\left.0.3 R_{*}\right)$. They are therefore likely to represent active region magnetic fields, although under this assumption a quite small filling factor of $6 \%$ is obtained (cf. Favata et al. 2000b for details).

Applications have also often shown that considerable heating rates are present during the decay phase of large stellar flares (e.g., Favata et al. 2000a,b). Values of $\zeta$ as small as 0.5 are frequently found; this corresponds to a decay that is $\approx 3-5$ times slower than predicted from free cooling on the thermodynamic time scale.

\subsection{Two-Ribbon flare models}

An approach that is entirely based on continuous heating (as opposed to cooling) was developed for the two-ribbon (2-R) class of solar flares. An example of this flare type is shown in Fig. 24. The 2-R flare model devised initially by Kopp and Poletto (1984) is a parameterized magnetic-energy release model. The time development of the flare light-curve is completely determined by the amount of energy available in non-potential magnetic fields, and by the rate of energy release as a function of time and geometry as the fields reconnect and relax to a potential-field configuration. Plasma cooling is not included in the original model; it is assumed that a portion of the total energy is radiated into the observed X-ray band, while the remaining energy will be lost by other mechanisms. An extension that includes approximations to radiative and conductive losses was described in Güdel et al. (1999) and Güdel et al. (2004). 2-R flares are well established for the Sun (Sect. 12.1, Fig. 24); they often lead to large, long-duration flares that may be accompanied by mass ejections.

The magnetic fields are, for convenience, described along meridional planes on the star by Legendre polynomials $P_{n}$ of order $n$, up to the height of the neutral point; above this level, the field is directed radially, that is, the field lines are "open". As time proceeds, field lines nearest to the neutral line move inward at coronal levels and reconnect at progressively larger heights above the neutral line. The reconnection point thus moves upward as the flare proceeds, leaving closed magnetic-loop systems underneath. One 


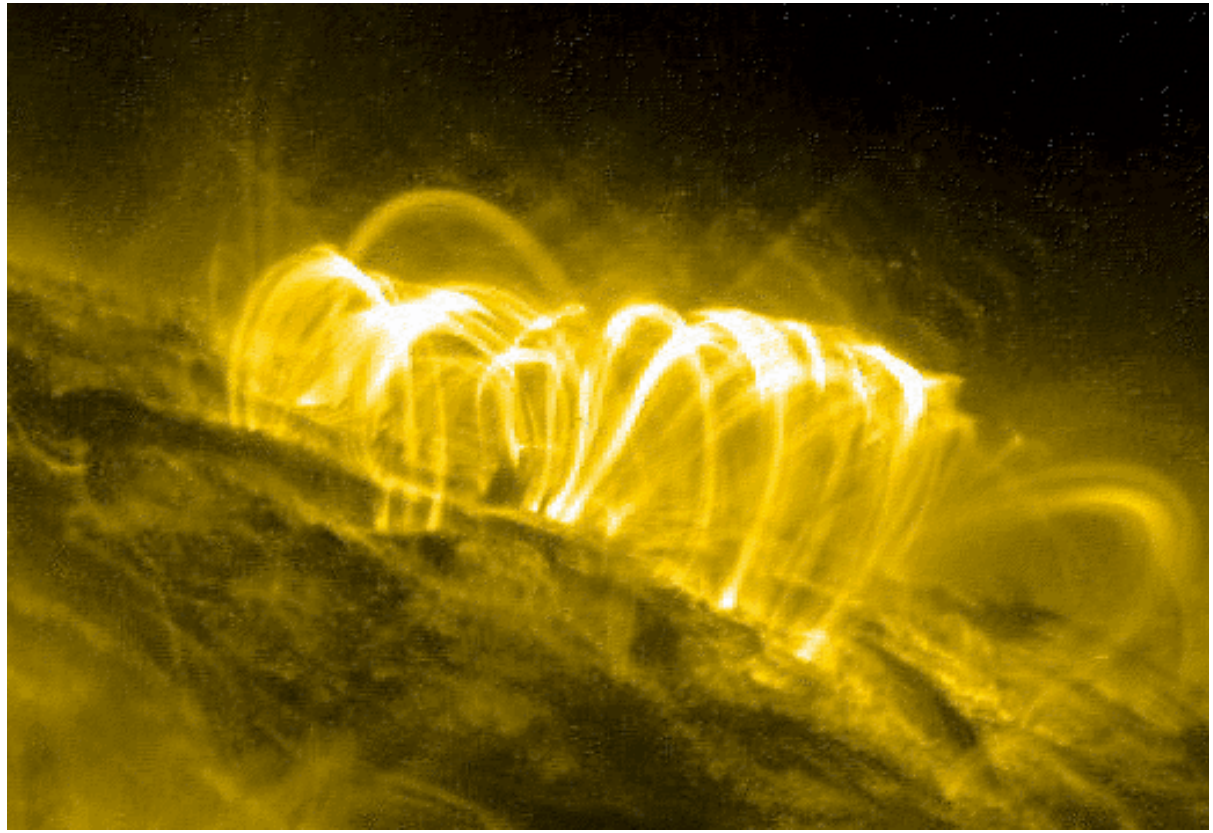

Fig. 24. Trace image of a flaring magnetic loop arcade

loop arcade thus corresponds to one N-S aligned lobe between two zeros of $P_{n}$ in latitude, axisymmetrically continued over some longitude in E-W direction. The propagation of the neutral point in height, $y(t)$, with a time constant $t_{0}$, is prescribed by ( $y$ in units of $R_{*}$, measured from the star's center)

$$
\begin{gathered}
y(t)=1+\frac{H_{\mathrm{m}}}{R_{*}}\left(1-e^{-t / t_{0}}\right) \\
H(t) \equiv[y(t)-1] R_{*}
\end{gathered}
$$

and the total energy release of the reconnecting arcade per radian in longitude is equal to the magnetic energy lost by reconnection,

$$
\begin{gathered}
\frac{\mathrm{d} E}{\mathrm{~d} y}=\frac{1}{8 \pi} 2 n(n+1)(2 n+1)^{2} R_{*}^{3} B^{2} I_{12}(n) \frac{y^{2 n}\left(y^{2 n+1}-1\right)}{\left[n+(n+1) y^{2 n+1}\right]^{3}} \\
\frac{d E}{d t}=\frac{d E}{d y} \frac{d y}{d t}
\end{gathered}
$$

(Poletto et al. 1988). In (48), $H_{\mathrm{m}}$ is the maximum height of the neutral point for $t \rightarrow \infty$; typically, $H_{\mathrm{m}}$ is assumed to be equal to the latitudinal extent of the loops, i.e.,

$$
H_{\mathrm{m}} \approx \frac{\pi}{n+1 / 2} R_{*}
$$

for $n>2$ and $H_{\mathrm{m}}=(\pi / 2) R_{*}$ for $n=2$. Here, $B$ is the surface magnetic field strength at the axis of symmetry, and $R_{*}$ is the stellar radius. Finally, $I_{12}(n)$ corresponds to 
$\int\left[P_{n}(\cos \theta)\right]^{2} d(\cos \theta)$ evaluated between the latitudinal borders of the lobe (zeros of $\left.\mathrm{d} P_{n} / \mathrm{d} \theta\right)$, and $\theta$ is the co-latitude.

The free parameters are $B$ and the efficiency of the energy-to-radiation conversion, $q$, both of which determine the normalization of the light curve; the time scale of the reconnection process, $t_{0}$, and the polynomial degree $n$ determine the duration of the flare; and the geometry of the flare is fixed by $n$ and therefore the asymptotic height $H_{\mathrm{m}}$ of the reconnection point. The largest realistic 2-R flare model is based on the Legendre polynomial of degree $n=2$; the loop arcade then stretches out between the equator and the stellar poles. Usually, solutions can be found for many larger $n$ as well. However, because a larger $n$ requires larger surface magnetic field strengths, a natural limit is set to $n$ within the framework of this model. Once the model solution has been established, further parameters, in particular the electron density $n_{e}$, can be inferred.

The Kopp and Poletto model is applicable after the initial flare trigger mechanism has terminated, although Pneuman (1982) suggested that reconnection may start in the earliest phase of loop structure development.

2-R models have been proposed for interpretation of stellar flares on phenomenological grounds such as high luminosities, long decay time scales, white-light transients, or X-ray absorption possibly by transits of prominences, by Haisch et al. (1981), Haisch et al. (1983), Poletto et al. (1988), Tagliaferri et al. (1991), Doyle et al. (1992b), Franciosini et al. (2001), Güdel et al. (1999), Güdel et al. (2004), to mention a few. Poletto et al. (1988) emphasized the point that large solar flares predominantly belong to this class rather than to compact, single-loop events. Many authors found overall sizes of order $R_{*}$, i.e., they obtained small $n$.

Comparisons of 2-R model predictions with explicitly measured heating rates during large flares indicate an acceptable match from the rise to the early decay phase (van den Oord and Mewe 1989) although the observed later decay episodes are often much slower than any model parameter allows (Osten et al. 2000; Kürster and Schmitt 1996; Güdel et al. 2004). Part of the explanation may be additional heating within the cooling loops.

Katsova et al. (1999) studied a giant long-duration flare on the dMe star AU Mic. The observed decay time of this flare exceeded any plausible decay time scale based on pure radiative cooling by an order of magnitude, suggestive of a 2-R flare. Although an alternative model based on a very large, expanding CME-like plasma cloud at low densities has been presented (Cully et al. 1994), the spectroscopically measured high densities of the hot plasma argue for a coronal source undergoing significant posteruptive heating. A very slowly evolving flare observed on the RS CVn-type binary HR 5110, with a decay time of several days, suggested large source scales but showed clear departures from any model fit; the authors proposed that the flare was in fact occurring in intrabinary magnetic fields (Graffagnino et al. 1995).

\subsection{Hydrodynamic models}

Full hydrodynamic calculations aim at solving the equations of mass, momentum, and energy conservation comprehensively and under quite general conditions, although certain simplifying initial conditions, boundary conditions, and plasma physical approximations must be made. The equations treat the plasma as a thermodynamic fluid, an approximation that is sufficiently good except for the critical footpoint region of magnetic loops 
where the mean free path of the electrons exceeds the temperature and pressure scale heights. In this region, effects such as saturation of the conductive flux due to the freestreaming limit to the heat transport must be included. Treatment of time-dependent ionization and recombination is also required at chromospheric levels. The latter define boundary conditions to the coronal simulations, therefore extensive chromospheric models must be included (see, e.g., Reale et al. 1988). Radiation can usually be approximated sufficiently well in terms of a cooling function integrating all continuum, free-bound, and line losses at a given temperature in ionization equilibrium. The presence of nonthermal, accelerated electron populations is often not treated, and the magnetic fields are assumed to be rigid, with the only purpose of confining the plasma. Heating terms are adjusted empirically, with a distribution both in space and time.

Accepting these approximations, state-of-the art simulations have given deep insights into the flare process and the nature of spatially unresolved stellar X-ray flares. The methods have been comprehensively tested with, and applied to, solar flares (Pallavicini et al. 1983; Reale and Peres 1995). For a description of various codes and numerical solar flare simulations, see Nagai (1980), Peres et al. (1982), Pallavicini et al. (1983), Peres et al. (1987), and Mariska et al. (1989). For stellar applications, I refer the reader to Cheng and Pallavicini (1991), Katsova et al. (1997), Reale et al. (2002), and Reale et al. (2004).

For stellar flare simulations, there are two major unknowns. First, the energy input can be in the form of high-energy particle beams or of direct heating, leading to different preferred heat source locations such as the footpoints or the loop apex. Fortunately, conduction equalizes effects due to spatially non-uniform heating rapidly enough so that the choice is of limited relevance (Peres et al. 1987; Reale et al. 1988). Second, the basic magnetic configuration of the flares is unknown a priori, including initial conditions in the pre-flare plasma such as pressure or density. Pure hydrodynamic models assume single flaring loops (e.g., Peres et al. 1982), or complexes of independent loops (e.g., Reale et al. 2004), while magnetohydrodynamic (MHD) codes allow for an extension to interacting loops or loop arcades (see below). By varying parameters, comparison of the simulations with observed parameters such as the light curve and the temperature development eventually confine magnetic loop geometries, magnetic fields, heating durations, electron densities, and some of the initial conditions.

A parameter study appropriate for flares on dMe stars was performed by Cheng and Pallavicini (1991). They pointed out that multiple solutions exist for given light curves, but that all solutions fulfill an overall linear relation between flare peak EM and total energy released in the flare, with good agreement between the simulations and the observations. This relation emerges because larger energy release produces more extensive chromospheric evaporation. The trend saturates, however, once the energy input becomes too large, because increased radiative losses suppress further conductive evaporation. This, then, suggests that extremely large flares cannot be produced in moderately-sized magnetic loops. The simulations further showed plasma evaporative upflows in the early episodes that reach velocities of $500-2000 \mathrm{~km} \mathrm{~s}^{-1}$. Such velocities can in principle be measured spectroscopically.

Reale et al. (1988, 2004) studied two giant flares on Proxima Centauri using hydrodynamic approaches. Both flares have been suggested to be close analogs of gradual, very large solar events, with the important difference that their geometric sizes $\left(5 \times 10^{9} \mathrm{~cm}-\right.$ 
$10^{10} \mathrm{~cm}$ ), while similar to large-scale solar events, comprise a significant fraction of the stellar corona. For the second flare, Reale et al. (2004) showed explicitly that multiple flaring loops are required to describe the light curve. This led them to suggest that the actual geometry should be a loop arcade in analogy to the 2-R model, of which a few dominant loops with similar heights were modeled like isolated flaring loops. The same flare modeled with the 2-R approach (Sect. 12.7) indeed leads to very similar magnetic loop heights (Güdel et al. 2004). Large flares on late-type M dwarfs therefore constitute large-scale disturbances of the corona possibly inducing global effects, an assertion that has been supported by interferometric radio observations (Benz et al. 1998).

\subsection{Magnetohydrodynamic models}

Shibata and Yokoyama (1999) and Shibata and Yokoyama (2002) discussed novel flare scaling laws based on their earlier MHD work on X-point reconnection in large flares. They reported a simple scaling relation between flare peak temperature $T$, the loop magnetic field strength $B$, the pre-flare loop electron density $n_{0}$, and the loop semilength $L$ under the condition of dominant conductive cooling (appropriate for the early phase of a flare),

$$
T \approx 1.8 \times 10^{4} B^{6 / 7} n_{0}^{-1 / 7} L^{2 / 7}[\mathrm{~K}] .
$$

The law follows from the balance between conduction cooling $\left(\propto T^{7 / 2} / L^{2},(32)\right)$ and magnetic reconnection heating $\left(\propto B^{3} / L\right)$. Assuming loop filling through chromospheric evaporation and balance between thermal and magnetic pressure in the loop, two further "pressure-balance scaling laws" follow:

$$
\begin{aligned}
& \mathrm{EM} \approx 3 \times 10^{-17} B^{-5} n_{0}^{3 / 2} T^{17 / 2}\left[\mathrm{~cm}^{-3}\right] \\
& \mathrm{EM} \approx 2 \times 10^{8} L^{5 / 3} n_{0}^{2 / 3} T^{8 / 3}\left[\mathrm{~cm}^{-3}\right] .
\end{aligned}
$$

An alternative scaling law applies if the density development in the initial flare phase is assumed to follow balance between evaporation enthalpy-flux and conduction flux, although the observational support is weaker,

$$
\mathrm{EM} \approx 1 \times 10^{-5} B^{-3} n_{0}^{1 / 2} T^{15 / 2}\left[\mathrm{~cm}^{-3}\right] .
$$

And third, a steady solution is found for which the radiative losses balance conductive losses. This scaling law applies to a steady loop,

$$
\mathrm{EM} \approx\left\{\begin{array}{lll}
10^{13} T^{4} L & {\left[\mathrm{~cm}^{-3}\right]} & \text { for } T<10^{7} \mathrm{~K} \\
10^{20} T^{3} L & {\left[\mathrm{~cm}^{-3}\right]} & \text { for } T>10^{7} \mathrm{~K}
\end{array}\right.
$$

and is equivalent to the RTV scaling law (20).

The advantage of these scaling laws is that they make use exclusively of the flarepeak parameters $T$, EM, $B$ (and the pre-flare density $n_{0}$ ) and do not require knowledge of the time evolution of these parameters. The models have been applied to flares on protostars and T Tau stars (see Sect. 12.12). 


\subsection{Summary of methods}

Despite their considerable sophistication, stellar flare models remain crude approximations so long as we have little a priori knowledge of the magnetic field topology. Solar flares reveal complexities that go far beyond any of the standard models described above. Nevertheless, stellar flare scenarios have been useful tools to roughly assess characteristic flare sizes, densities, and heating rates. Several models have been applied to solar flares as well, which has tested their reliability.

In some cases, simple light curve decay analysis (35) or the quasi-static cooling model result in excessively large loop semi-lengths $L$ (Schmitt 1994; Favata and Schmitt 1999; Favata et al. 2000a). Alternative methods such as the heating-decay model (Sect. 12.6) may give more moderate values. On the other hand, seemingly different methods may also result in overall agreement for the magnetic structure size. For example, Endl et al. (1997) compared the 2-R approach with the quasi-static cooling formalism for a large flare on an RS CVn binary and found similar heights of the flaring structures $\left(\approx 1 R_{*}\right)$. Güdel et al. (2004) and Reale et al. (2004) compared the 2-R model, full hydrodynamic simulations and the heating-decay model, again finding good overall agreement (loop sizes of order $1 R_{*}$ on Proxima Centauri). Covino et al. (2001) compared loop lengths obtained for several recently-modeled large stellar flares based on a simple decay-time formalism (35) and on the heating-decay model; the agreement was once more rather good. The authors argued that neglecting heating during the decay increases the model length in the decay-time formalism, but at the same time one ignores conductive cooling. The density is thus overestimated, which decreases the model loop size again. In this sense, approaches such as the heating-decay model or the analytic 2-R model are preferred not necessarily (only) because of their predictive power but because of their physically founded basis and hence reliability, and their support from direct solar observations. Full hydrodynamic or MHD simulations of course provide the closest description of the actual processes, but a realistic simulation requires a careful choice of a number of unknown parameters.

One of the main results that have come from extensive modeling of stellar X-ray flares is that extremely large stellar flares require large volumes under all realistic assumptions for the flare density. This is because, first, the energy derives from the non-potential portion of the magnetic fields that are probably no stronger than a few $100 \mathrm{G}$ in the corona; and second, small-loop models require higher pressure to produce the observed luminosity, hence requiring excessively strong magnetic fields. This is in line with the findings by Cheng and Pallavicini (1991) from hydrodynamic simulations. This is not to say that magnetic loops must be of enormous length - a number of interpretation methods suggest the contrary even for very large flares. But at this point, the concept of single-loop approaches becomes questionable particularly as large flares on the Sun often involve very complex arrangements of magnetic structures: the large volumes do not necessarily involve large heights but large surface area (see example in Reale et al. 2004).

Alternative models are available in the stellar literature, although they have mostly been applied to singular cases. Most notably, I mention the coronal mass ejection model by Cully et al. (1994) that was applied to a giant flare on AU Mic observed by EUVE (Cully et al. 1993). Fisher and Hawley (1990) derived equations for the evolution of a constant-cross section flaring magnetic loop with uniform but time-varying pressure and 
volumetric heating rate (for time scales that are long compared to the sound transit time, i.e., assuming quasi-hydrostatic equilibrium). Although their model is not quasi-static by requirement, it can be well approximated by "equivalent static loops" having the same length and the same column depth as the evolving loop. A major advantage of this formulation is that it includes the evaporation phase of the flare, i.e., essentially the flare rise phase. The quality of the model was described in detail by Fisher and Hawley (1990), together with applications to a solar and an optical stellar flare. Another application was described by Hawley et al. (1995) for an EUV flare on AD Leo. Table 4 reports the refined results presented by Cully et al. (1997) for a typical low-abundance corona. A further model including evaporative cooling was presented by Pan et al. (1997) in an application to a strong flare on an M dwarf.

There are a number of further, "unconventional" models, e.g., star-disk magnetic flares in pre-main sequence stars or intrabinary flares - see, among others, Skinner et al. (1997) and Montmerle et al. (2000).

\subsection{Observations of stellar X-ray flares}

Table 4 summarizes properties of a list of stellar X-ray events that have been modeled with one of the above approaches in the literature (I have added a few selected published flares for which modeling is not available but for which good temperature and EM measurements have been given). Further flares on pre-main sequence stars have been discussed by Imanishi et al. (2003). Some flares have reported durations of several days (Graffagnino et al. 1995; Kürster and Schmitt 1996; see also Ayres et al. 1999, 2001a), some show X-ray luminosities up to $10^{33} \mathrm{erg} \mathrm{s}^{-1}$ (e.g., Preibisch et al. 1995; Tsuboi et al. 1998), and some show temperatures in excess of 100 MK (Tsuboi et al. 1998; Güdel et al. 1999; Favata and Schmitt 1999). A number of extreme flares were already reported from the pre-Einstein era, with RS CVn binaries recognized as their origin (Pye and McHardy 1983). Unusual shapes of flares have frequently been reported, such as flares with slow rise and rapid decay (Haisch et al. 1987), flares with secondary peaks or separate reheating events during the primary decay (White et al. 1986; Katsova et al. 1999; Güdel et al. 2004), flares with double-exponential decays (Cully et al. 1993; Osten and Brown 1999; Favata et al. 2000a; Reale et al. 2004, see Fig. 25), flat-topped flare light-curves (Agrawal et al. 1986b; Osten and Brown 1999; Raassen et al. 2003b, see Fig. 25), flares with very long rise times (Tagliaferri et al. 1991), or flares in which the temperature stays essentially constant during the decay phase (Graffagnino et al. 1995).

\subsection{Flare temperatures}

When the flare energy release evaporates plasma into the corona, heating and cooling effects compete simultaneously, depending on the density and temperature profiles in a given flare. It is therefore quite surprising to find a broad correlation between peak temperature $T_{p}$ and peak emission measure $\mathrm{EM}_{p}$, as illustrated in Fig. 26 for the sample reported in Table $4 .{ }^{5}$ A regression fit gives (for 66 entries)

5 If ranges are given in the table, the geometric mean of the minimum and maximum was taken; upper or lower limits were treated as measured values. 


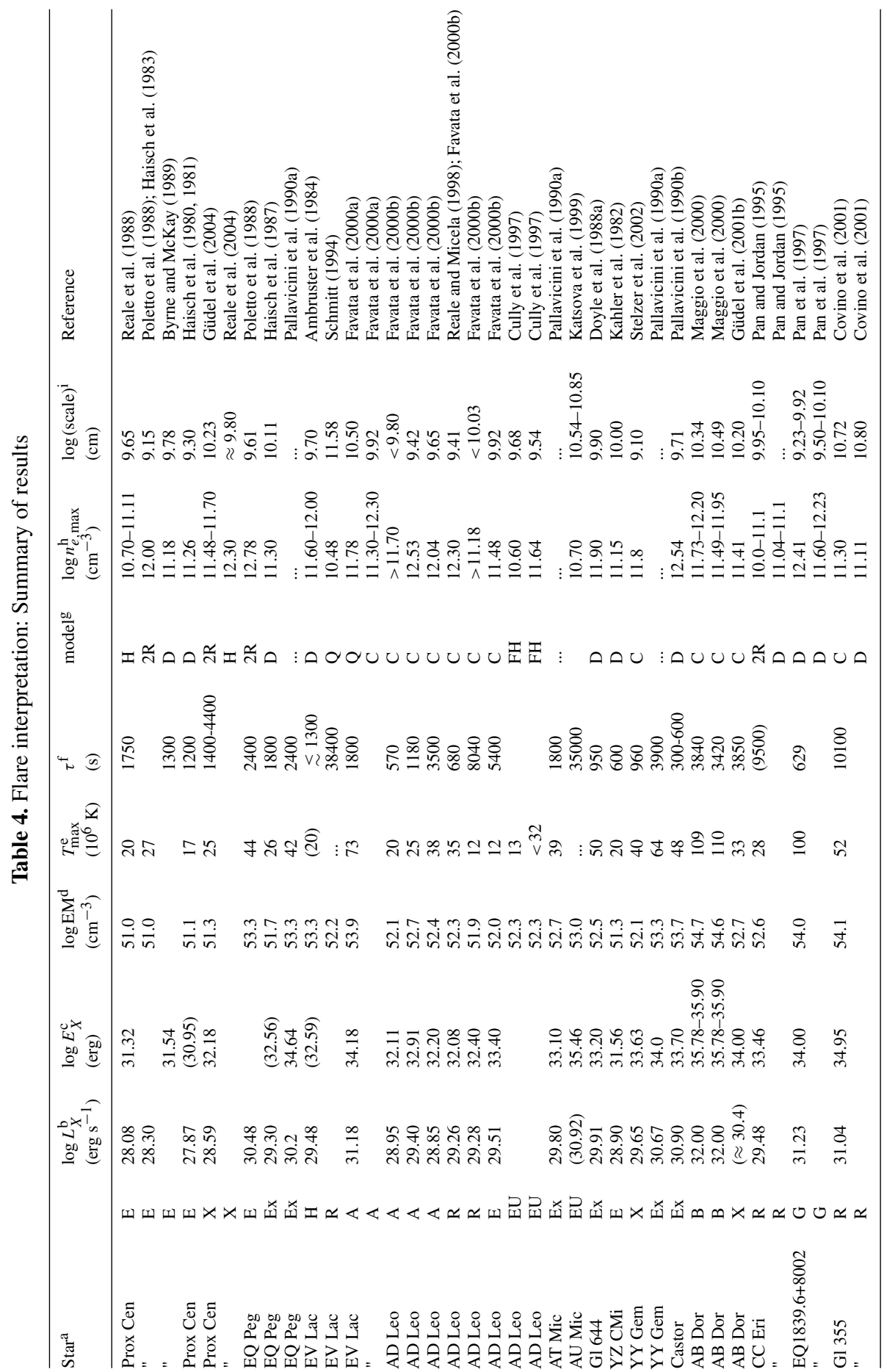




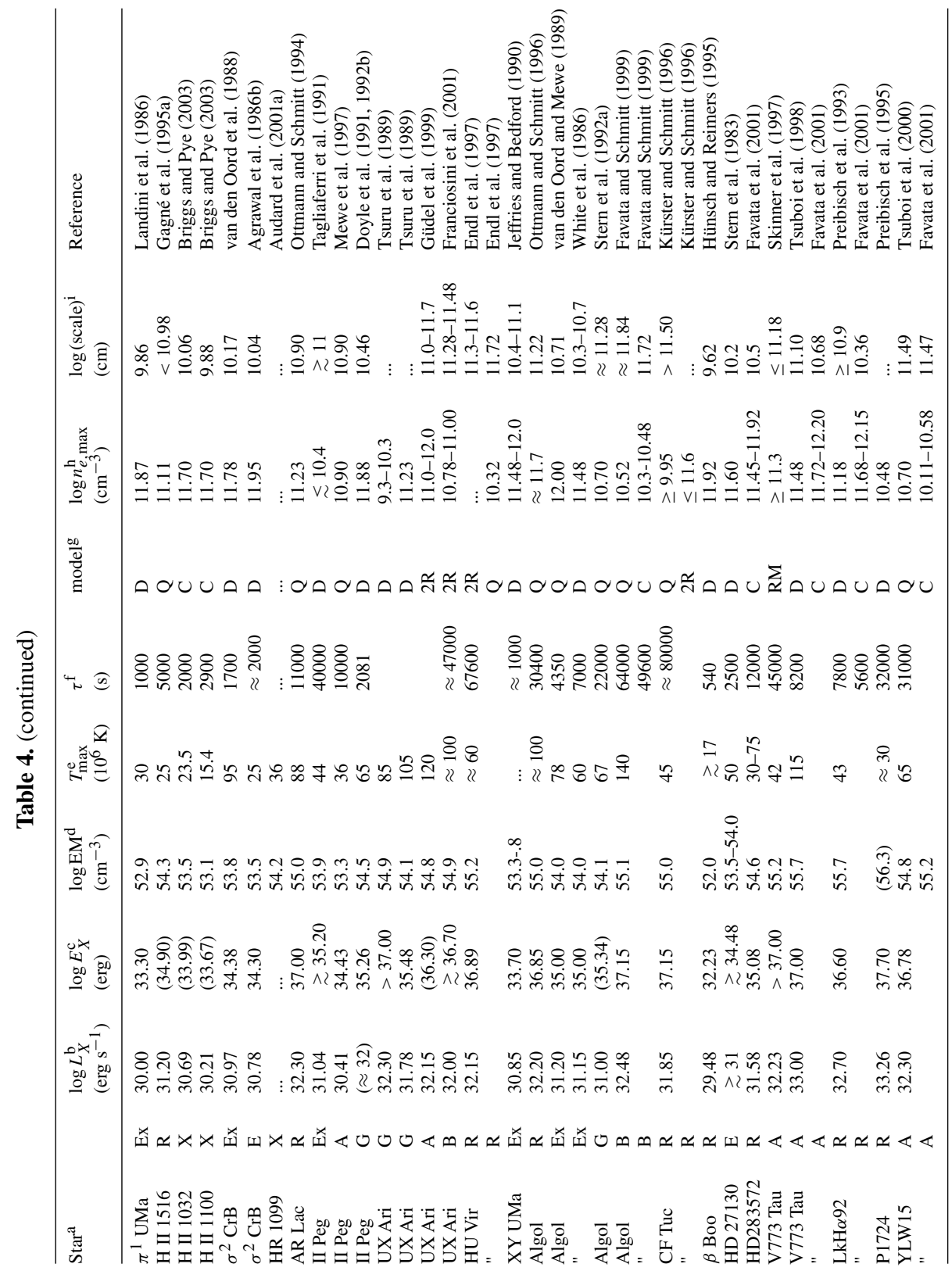




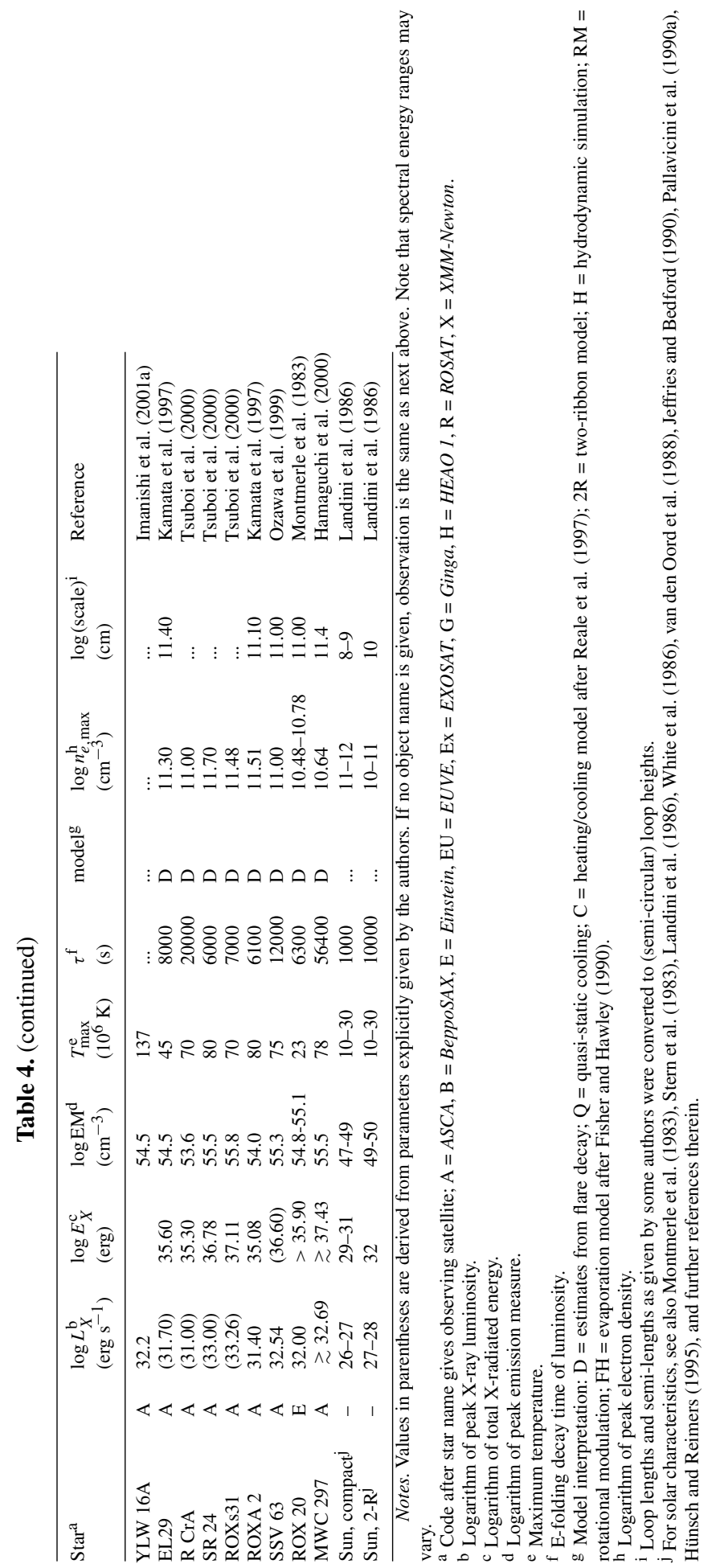



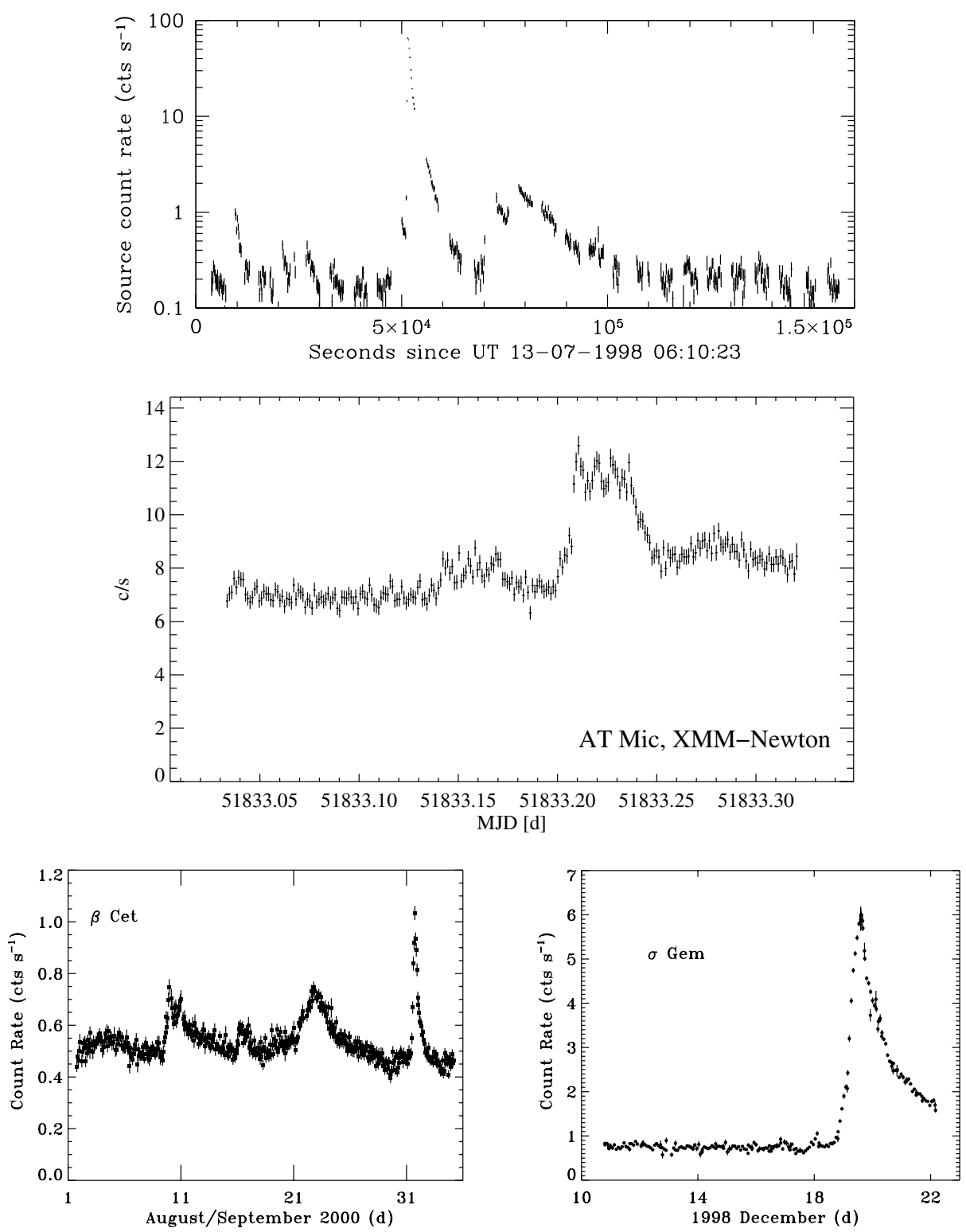

Fig. 25. Four largely different examples of stellar flares. Top: A very large flare on EV Lac, showing a rapid rise and a long double-exponential decay (figure courtesy of F. Favata, after Favata et al. 2000a, observations with ASCA GIS.) - Middle: Modest, flat-topped flare on AT Mic (from Raassen et al. 2003b, observations with XMM-Newton EPIC). Bottom left: Sequence of very slowly decaying flares on the giant $\beta$ Cet (figure courtesy of R. Osten, after Ayres et al. 2001a, observations with EUVE). Bottom right: Rapid rise and very slow decay of a flare on the RS CVn binary $\sigma$ Gem (figure courtesy of R. Osten, observations with EUVE) 


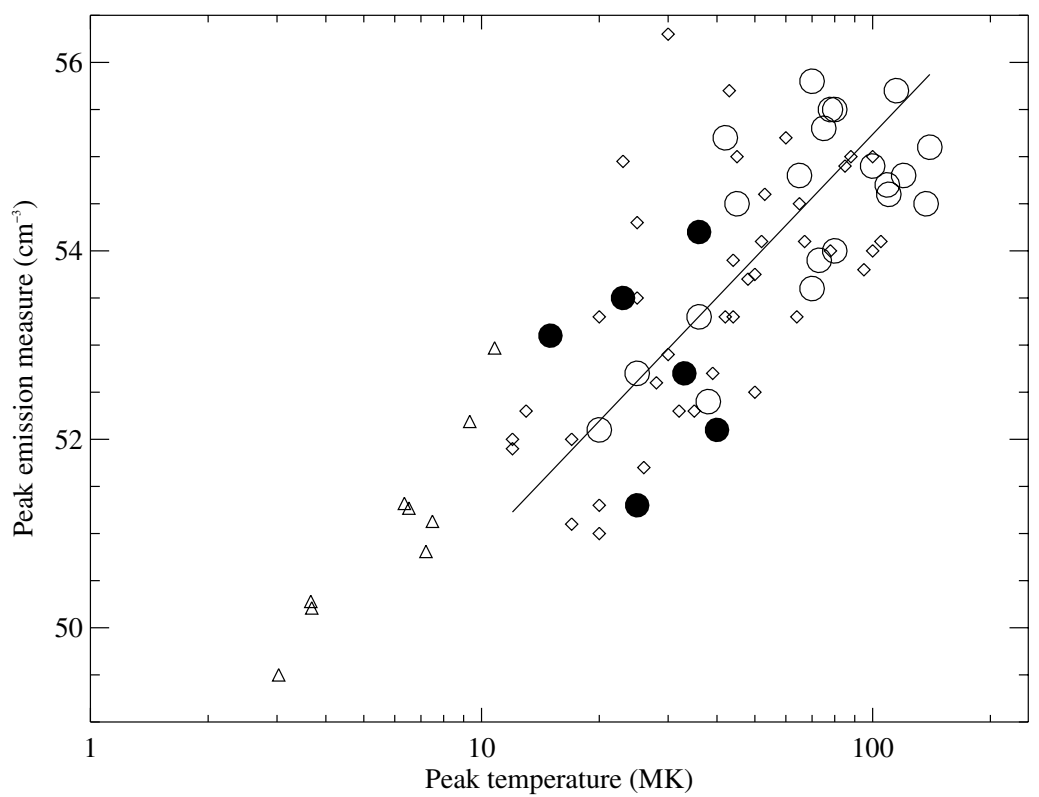

Fig. 26. Peak temperatures and EMs of the flares listed in Table 4. Key to the symbols: Filled circles: XMM-Newton observations. Open circles: ASCA or BeppoSAX observations. Small diamonds: observations from other satellites. The solid line shows a regression fit (58). Triangles represent non-flaring parameters of the G star sample from Güdel et al. (1997a) and Güdel et al. (1998), referring to the hotter plasma component in 2-T spectral fits to ROSAT data

$$
E M_{p} \propto T_{p}^{4.30 \pm 0.35}
$$

The correlation overall indicates that larger flares are hotter. A similar relation was reported previously for solar flares (Feldman et al. 1995), with a suggestion that it extends to selected large stellar flares. Although the trend shown in Fig. 26 does not smoothly connect to the Feldman et al. solar-flare relation (theirs being lower in EM or hotter for a given EM, see also Fig. 27), their stellar loci agree with ours. Various selection effects or data analysis biases related to limited $\mathrm{S} / \mathrm{N}$ ratios and detector energy resolution may prohibit an accurate comparison of the two relations. Also, Feldman et al. (1995) measured the temperature at the time when the EM reached its peak, whereas Table 4 reports the maximum temperature that often occurs slightly before the EM peak. Consideration of this effect could only increase the disagreement. A handful of stellar flares for which the temperature was determined precisely at the EM peak yield the same slope as given in (58).

On the other hand, the correlation is reminiscent of the $T-L_{X}$ correlation for the "non-flaring" coronal stars in Fig. 10 at cooler temperatures. This same sample is plotted as triangles in Fig. 26, again only for the hotter plasma component (data from Güdel et al. 1997a). The stars follow approximately the same slope as the flares, albeit at cooler temperatures, and for a given temperature, the EM is higher. This trend may suggest that flares systematically contribute to the hot plasma component, although we have not temporally averaged the flare temperature and EM for this simple comparison. For this 


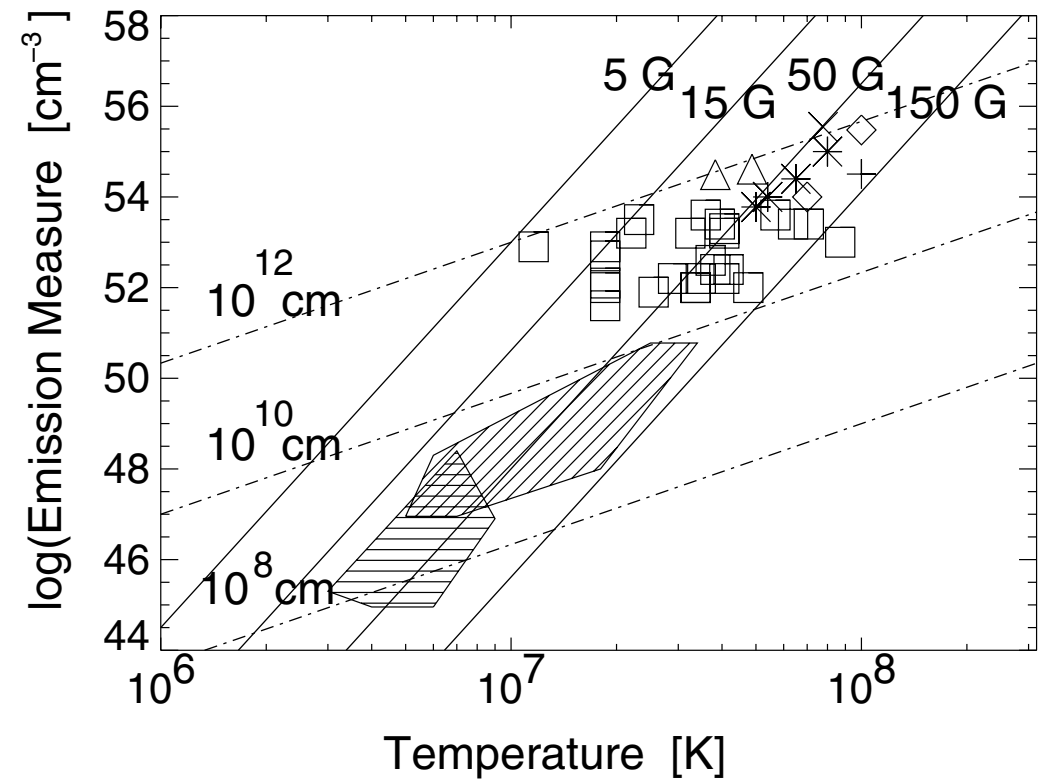

Fig. 27. Theoretical EM- $T$ relations based on the reconnection model by Shibata and Yokoyama, showing lines of constant loop length $L$ and lines of constant magnetic field strength $B$. Hatched areas are loci reported for solar flares, and other symbols refer to individual stellar flares in starforming regions (figure courtesy of K. Shibata and T. Yokoyama, after Shibata and Yokoyama 2002)

model, we would require that the coronal emission of more luminous stars is dominated by larger and hotter flares. If flares are distributed in energy as described in Sect. 13.5, then a larger number of flares will generate both a higher luminosity and a shift to higher average temperatures indeed.

Shibata and Yokoyama (1999) and Shibata and Yokoyama (2002) interpreted the EM- $T$ relation as presented by Feldman et al. (1995) based on their MHD flare scaling laws (54). The observed loci of the flares require loop magnetic field strengths similar to solar flare values $(B \approx 10-150 \mathrm{G}$ ) but the loop lengths must increase toward larger flares. This is seen in Fig. 27 where lines of constant $L$ and $B$ are plotted for this flare model. The same applies to the flares in Fig. 26; typical loop lengths would then be $L \approx 10^{11} \mathrm{~cm}$.

\subsection{Flare densities}

The density of a flaring plasma is of fundamental importance because it determines the time scales of radiation and of several plasma-physical instabilities. For spatially unresolved observations, densities can be inferred either indirectly from a flare decay analysis (see Sect. 12.4-12.7) or directly by measuring density-sensitive line ratios as described in Sect. 10.

Good examples from solar studies are relatively rare. McKenzie et al. (1980) presented O vII He-like triplets (Sect. 10.2) observed during a large solar flare and found 
$f / i$ ratios around unity close to the flare peak, implying densities of up to $2 \times 10^{11} \mathrm{~cm}^{-3}$. Much shorter flares were discussed by Doschek et al. (1981); in those cases, the densities reached peaks around $(10-20) \times 10^{11} \mathrm{~cm}^{-3}$ as measured from $\mathrm{O}$ vII, but this occurred during the flare rise, while electron densities of a few times $10^{11} \mathrm{~cm}^{-3}$ were derived during the flare peak. The estimated masses and volumes, on the other hand, steadily increased. For the more relevant hot temperatures, line ratio diagnostics based on Fe XXI, Fe XXII, or (He-like) Fe XXv have been employed. Doschek et al. (1981) and references therein reported densities derived from Fe Xxv $(T>10 \mathrm{MK})$ that are similar to those measured from O viI. Phillips et al. (1996) inferred very high densities of $10^{13} \mathrm{~cm}^{-3}$ from Fe XXII about one minute after the Ca XIX flare peak, and $n_{e} \approx(2-3) \times 10^{12} \mathrm{~cm}^{-3}$ from Fe XXI five minutes later. Landi et al. (2003) recently used various density diagnostics for a modest solar limb flare. From Fe XXI lines, they derived densities of up to $3 \times 10^{12} \mathrm{~cm}^{-3}$, but there are conflicting measurements for lower ionization stages that reveal much lower densities, comparable to pre-flare values (see also further references in their paper). If the density values at $\approx 10^{7} \mathrm{~K}$ are real, then pressure equilibrium cannot be assumed for flaring loops; a possible explanation involves spatially separate volumes for the OVII and the Fe XXI-XXV emitting plasmas.
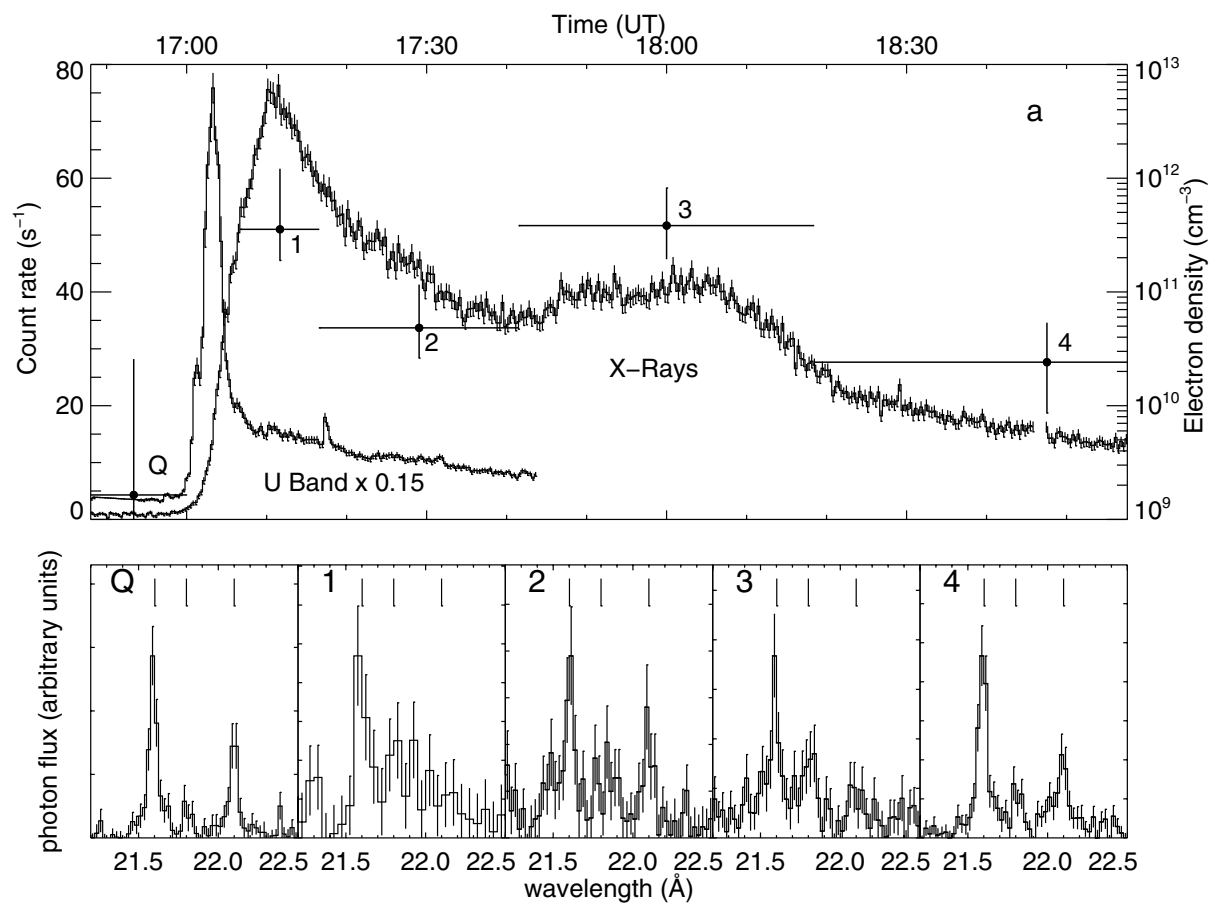

Fig. 28. Flare on Proxima Centauri, observed with $X M M$-Newton. The top panel shows the X-ray light curve and the much shorter U band flare (around 17 UT). The bottom panel shows the O VII He-like triplets observed during various time intervals of the flare. The locations of the $r, i$, and $f$ lines are marked by vertical lines. The resulting electron densities are given in the top panel by the crosses, where the horizontal arm lengths indicate the time intervals over which the data were integrated, and the right axis gives the logarithmic scale (after Güdel et al. 2002a, 2004) 
Stellar flare density measurements are in a rather infant state as they require high signal-to-noise ratios over the short time of a flare, and good contrast against the steady stellar emission. A large flare on Proxima Centauri provided first evidence for significant density variations as derived from the He-like O vir triplet (and more tentatively, from Ne IX; Güdel et al. 2002a, 2004, Fig. 28). The densities rapidly increased from $n_{e}<$ $10^{10} \mathrm{~cm}^{-3}$ to $\approx 4 \times 10^{11} \mathrm{~cm}^{-3}$ at flare peak, then again rapidly decayed to $\approx 2 \times$ $10^{10} \mathrm{~cm}^{-3}$, to increase again during a secondary peak, followed by a gradual decay. The instantaneous mass involved in the cool, O vII emitting source was estimated at $\approx 10^{15} \mathrm{~g}$, resulting in similar (instantaneous) potential and thermal energies in the cool plasma, both of which are much smaller than the total radiated X-ray energy. It is probable that the cool plasma is continuously replenished by the large amount of material that is initially heated to higher temperatures and subsequently cools to O VII forming temperatures and below. The measured densities agree well with estimates from hydrodynamic simulations (Reale et al. 2004). A marginal signature of a density increase was also recorded in O VII during a modest flare on YY Gem (Stelzer et al. 2002), and in Mg XI during a flare on $\sigma^{2} \mathrm{CrB}$ although the density stayed similarly high outside the flare (Osten et al. 2003). Further marginal indications for increased densities during flares were reported for AD Leo (van den Besselaar et al. 2003), AT Mic (Raassen et al. 2003b), and AU Mic (Magee et al. 2003).

Measurements from Fe line ratios in the EUV have been hampered by the apparently rather large quiescent densities that already reach values expected for flares (see Sect. 10.1). They therefore generally show little evidence for density increases during flares (Osten and Brown 1999). Monsignori Fossi et al. (1996) inferred $n_{e}$ up to $1.5 \times 10^{13} \mathrm{~cm}^{-3}$ from from the Fe XXI $\lambda 142.2 / \lambda 128.7$ flux ratio during a giant flare on AU Mic although the detection is again marginal. Sanz-Forcada et al. (2001) and SanzForcada et al. (2002) found slightly higher densities during flares on $\lambda$ And, HR 1099, and $\sigma$ Gem based on Fe XXI compared to quiescence $\left(\log n_{e} \approx 12.9\right.$ vs. 12.1 in $\lambda$ And), but again all values are extremely high.

\subsection{Correlation with UV and optical flares}

Optical continuum flares ("white light flares"), often observed in the Johnson U band, are tracers of the impulsive phase of the flare. The emission is presumably due to enhanced continuum emission after chromospheric heating, possibly following electron impact (e.g., Hawley et al. 1995). In the chromospheric evaporation scenario, U band bursts are expected to occur during the rise phase of soft X-ray flares (Sect. 12.16). Early examples were reported by Kahler et al. (1982), de Jager et al. (1986), and de Jager et al. (1989) for flares on YZ CMi, BY Dra, and UV Cet, respectively. The total optical energy output is approximately $0.1-1$ times the X-ray output, a range that has been confirmed by many other observations, including solar data (Kahler et al. 1982 and references therein). The flare amplitudes and radiated energies appear to be correlated in the X-ray and UV ranges (Mitra-Kraev et al. 2004).

Occasionally, very good correlations are found between soft X-ray and H $\alpha$ flares. The total energy emitted in $\mathrm{H} \alpha$ amounts to approximately $5 \%$ of the soft X-ray losses (Doyle et al. 1988a) but may, in exceptional cases, reach order unity (Kahler et al. 1982). The physical causes of this correlation were discussed by Butler (1993); direct 
photoionization of chromospheric material by coronal soft X-rays is problematic because the $\mathrm{H} \alpha$ ribbons seen on the Sun are narrowly confined to the magnetic footpoints, and the correlation is not necessarily detailed in time. A more likely explanation involves the same electrons that also induce the continuum white light flare in the chromosphere, as discussed above.

In some cases, however, optical and X-ray flares may be uncorrelated. Haisch et al. (1981) described an X-ray flare without an accompanying signal in the optical or the UV. Vice versa, Doyle et al. (1986) and Doyle et al. (1988b) reported $\mathrm{H} \gamma$ and U band flares on YZ CMi with no indication of a simultaneous response in soft X-rays. They suggested that the heating occurred in low-lying loops in the transition region that did not reach coronal levels; alternatively, absorption of the X-rays by cold overlying material is a possibility, or heating by proton beams that are more efficient at inhibiting chromospheric evaporation.

\subsection{Correlation with radio flares}

Similar to the case of $U$ band flares, we expect that non-thermal radio events, produced by accelerated particles, precede X-ray flares. Reports on such correlations have been rather mixed. Kundu et al. (1988) described a poor correlation between X-ray events and radio flares observed during an EXOSAT-VLA joint survey program of dMe stars, although there may be contemporaneous flaring in the two wavelength regions (see also Kahler et al. 1982). Since this program was carried out at relatively long radio wavelengths $(6 \mathrm{~cm}$ and $20 \mathrm{~cm})$, the observed radio bursts were probably produced by a coherent emission process that requires relatively few electrons in an unstable energy distribution. We cannot expect a one-to-one correspondence in time for those cases. A special diagnostic case of correlated behavior can be observed for gyrosynchrotron radio flares at higher radio frequencies - see below.

\subsection{The "Neupert Effect"}

Radio gyrosynchrotron, hard X-ray, and optical emissions are induced on time scales of the electron propagation (seconds), and therefore essentially develop proportionally to the influx of high-energy particles if long-term trapping does not occur. On the other hand, the cooling time of a thermal plasma in an extended coronal loop is governed by radiation and conduction with typical time scales of several minutes to hours. The $\mathrm{X}$-ray radiation therefore develops roughly proportionally to the accumulating thermal coronal energy ${ }^{6}$. To first order thus, for the radio $(\mathrm{R})$, optical $(\mathrm{O})$, and hard X-ray (HXR) luminosities,

$$
L_{\mathrm{R}, \mathrm{O}, \mathrm{HXR}}(t) \propto \frac{d}{d t} L_{\mathrm{X}}(t),
$$

\footnotetext{
${ }^{6}$ We ignore the detailed evolution of the flare temperature and the density and thus the EM; the evolution of $T$ and $n_{e}$ may even be coupled, see Sect. 12.6; strictly speaking, in (59) one should refer to the thermal energy in the hot plasma rather than to the X-ray luminosity which may not be proportional to the former (see Güdel et al. 1996).
} 
a relation that has first been formulated for solar radio and X-ray flares (Neupert 1968) and that has become known as the "Neupert Effect". It is a good diagnostic for the chromospheric evaporation process and has been well observed on the Sun in most impulsive and many gradual flares (Dennis and Zarro 1993). The search for stellar equivalents has been more challenging.

Hawley et al. (1995) observed the dMe star AD Leo in the EUV (as a proxy for X-rays) and in the optical during extremely long gradual flares. Despite the long time scales and considerable time gaps in the EUV observations, the presence of the Neupert relation (59) was demonstrated (in its integrated form).

Güdel et al. (1996) observed the similar dMe binary UV Cet during several shorter flares in X-rays and at radio wavelengths. A Neupert dependence between the light curves was clearly evident and was found to be very similar to the behavior of some gradual solar flares. Furthermore, the ratio between the energy losses in the two energy bands was derived to be similar to the corresponding luminosity ratio "in quiescence". Similar results apply to RS CVn binaries: Osten et al. (2004) reported the Neupert effect in X-ray, EUV, and radio observations of HR 1099, again with radiative X-ray/radio energy ratios that are close to quiescent conditions.

An example linking X-rays with white-light emission was presented by Güdel et al. (2002a) in observations obtained with XMM-Newton. The flare is illustrated in Fig. 28. Equation (59) is closely followed by the two light curves during the early part of the flare. The same temporal behavior was identified in a sequence of small flares during the same observation, suggesting that a considerable fraction of the low-level radiation is induced by temporally overlapping episodes of chromospheric evaporation.

Although the relative timing provides important support for the evaporation model, the absolute energy content in the fast electrons must also be sufficient to evaporate and heat the observed plasma. Given the uncertain nature of the optical white-light flares, the energy content of the high-energy particles is difficult to assess. The situation is somewhat better at radio wavelengths although the spectral modeling of the non-thermal electron population is usually rather incomplete and order-of-magnitude. During a pair of gradual soft X-ray flares observed on the RS CVn binary $\sigma$ Gem (Güdel et al. 2002b), (59) was again followed very closely, suggesting that electrons are capable of heating plasma over extended periods. The total injected electron energy was found to equal or possibly largely exceed the associated X-ray losses albeit with large margins of uncertainty. Essentially all of the released energy could therefore initially be contained in the fast electrons.

Similar timing between radio and X-ray flare events is seen in previously published light curves, although the Neupert effect was not discussed. Notable examples include flares described by Vilhu et al. (1988), Stern et al. (1992b), Brown et al. (1998), and Ayres et al. (2001b). If optical emission is taken as a proxy for the radio emission, further examples can be found in Doyle et al. (1988b), Kahler et al. (1982), de Jager et al. (1986), and de Jager et al. (1989). The Neupert effect is observed neither in each solar flare (50\% of solar gradual flares show a different behavior; Dennis and Zarro 1993), nor in each stellar flare. Stellar counter-examples include an impulsive optical flare with following gradual radio emission (van den Oord et al. 1996), gyrosynchrotron emission that peaks after the soft X-rays (Osten et al. 2000), an X-ray depression during strong radio flaring 
(Güdel et al. 1998), or the absence of any X-ray response during radio flares (Fox et al. 1994; Franciosini et al. 1999).

\subsection{Non-thermal hard X-rays?}

In solar flares, non-thermal hard X-rays begin to dominate the spectrum beyond 15$20 \mathrm{keV}$. Typically, these spectral components are power laws since the electron distributions in energy are power laws (Brown 1971). An ultimate test of the flare evaporation scenario in large flares on magnetically active stars would consist in the detection of non-thermal hard X-ray components during the soft X-ray flare rise. Observations with Ginga up to $\approx 20 \mathrm{keV}$ initially seemed to suggest the presence of such emission in quiescence (Doyle et al. 1992a) although Doyle et al. (1992b) showed that an unrealistically large number of electrons, and therefore an unrealistically large rate of energy release, would be involved. A continuous emission measure distribution with a tail up to very high temperatures, $T \gtrsim 10^{8} \mathrm{~K}$, can explain the data self-consistently, in agreement with previous arguments given by Tsuru et al. (1989).

Up to the present day, no compelling evidence has been reported for non-thermal $\mathrm{X}$-rays from stellar coronae. The Phoswich Detector System (PDS) instrument on board BeppoSAX was sensitive enough to detect photons up to $50-100 \mathrm{keV}$ during large stellar flares, but all recorded spectra could be modeled sufficiently well with thermal plasma components. Examples include flares on UX Ari (Franciosini et al. 2001), AR Lac (Rodonò et al. 1999), Algol (Favata and Schmitt 1999), and AB Dor (Pallavicini 2001). The case of UX Ari is shown in Fig. 29. Although the PDS signal was strongest just

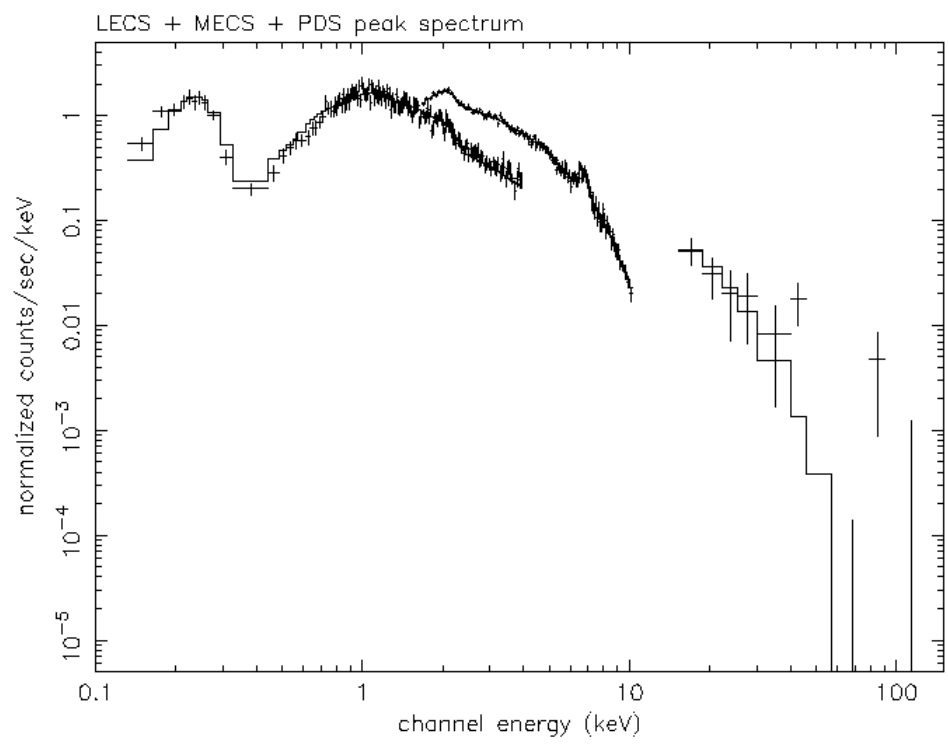

Fig. 29. X-ray spectra taken during a large flare on UX Ari by three detectors on board BeppoSAX. The histogram represents a fit based on thermal plasma components; it describes the data acceptably well up to about $40 \mathrm{keV}$ (figure courtesy of E. Franciosini, after Franciosini et al. 2001) 
before the early peak phase of the flare when non-thermal contributions are indeed expected, this is also the phase when the hottest plasma is formed, so that the light curves make no strong argument in favor of non-thermal emission either. The same applies to observations of giant flares on AB Dor (Pallavicini 2001).

An interesting suggestion for non-thermal contributions was put forward by Vilhu et al. (1993): the small equivalent width of the Fe $\mathrm{K}$ line at $6.7 \mathrm{keV}$ during flares on $\mathrm{AB}$ Dor could be due to a continuum level that is enhanced by an additional non-thermal (power-law) component. The principal uncertainty is the location of a lower cut-off in the electron distribution and consequently the turnover in the non-thermal spectral contribution. Model calculations predict that this view is tenable only if the magnetic fields are weaker than $50 \mathrm{G}$ (Vilhu et al. 1993).

The elusive non-thermal X-ray components are a classic case for the absence of evidence not giving any evidence of absence: to the contrary, the very efficient production of non-thermal radio emission in many of these active coronae both during flares and during (putative) "quiescence" is clear proof that large numbers of high-energy electrons are present in active coronae. We need more sensitive detectors to trace their radiative signatures, which will hold unparalleled information on the primary energy release in stellar coronae.

\section{The statistics of flares}

The study of coronal structure confronts us with several problems that are difficult to explain by scaling of solar coronal structure: i) Characteristic coronal temperatures increase with increasing magnetic activity (Sect. 9.5). ii) Characteristic coronal densities are typically higher in active than in inactive stars (Sect. 10), and pressures in hot loops can be exceedingly high (Sect. 11.2.2). iii) The maximum stellar X-ray luminosities exceed the levels expected from complete coverage of the surface with solar-like active regions by up to an order of magnitude (Sect. 11.4). iv) Radio observations reveal a persistent population of non-thermal high-energy electrons in magnetically active stars even if the lifetime of such a population should only be tens of minutes to about an hour under ideal trapping conditions in coronal loops (Güdel 2002) and perhaps much less due to efficient scattering of electrons into the chromosphere (Kundu et al. 1987). Several of these features are reminiscent of flaring, as are some structural elements in stellar coronae. If flares are important for any of the above stellar coronal properties indeed, then we must consider the effects of frequent flares that may be unresolved in our observations but that may make up part, if not all, of the "quiescent" emission.

\subsection{Correlations between quiescent and flare emissions}

In 1985, three papers (Doyle and Butler 1985; Skumanich 1985; Whitehouse 1985) reported an unexpected, linear correlation between the time-averaged power from optical flares and the low-level, "quiescent" X-ray luminosity. This correlation could suggest that the mechanism that produces the optical flares also heats the plasma, that is, the quasi-steady, slowly varying X-ray emission may be the product of stochastic flaring. Pearce et al. (1992) showed that over the entire solar magnetic cycle, the monthly average 
soft X-ray luminosity scales in detail and linearly with the rate of detected $\mathrm{H} \alpha$ flares, again supporting a picture in which a continuous distribution of flares at least contributes to the overall coronal heating. This is echoed in the observation that stellar "quiescent" luminosity correlates approximately linearly with the rate of X-ray flares (above some lower energy threshold; Audard et al. 2000, Fig. 33a below).

Güdel and Benz (1993) discussed a global relation between non-thermal radio luminosities of active stars and their "quiescent" X-ray luminosities. Since the short lifetime of $\mathrm{MeV}$ electrons in coronal magnetic fields implies frequent acceleration, a possible explanation again involves stochastic flares: the flare-accelerated electrons could themselves act as the heating agents via chromospheric evaporation. The smoking gun came with the observation that the total radio and X-ray outputs of solar flares follow the same correlation (Benz and Güdel 1994). Similarly, Haisch et al. (1990a) found that the ratio between energy losses in coronal X-rays and in the chromospheric Mg II lines is the same in flares and in quiescence. This also applies if UV-filter observations are used instead of Mg II fluxes (Mitra-Kraev et al. 2004). Finally, Mathioudakis and Doyle (1990) reported a tight correlation between $L_{X}$ and $\mathrm{H} \gamma$ luminosity that it the same for flares and for "quiescence". These observations point to an intimate relation between flares and the overall coronal emission.

\subsection{Short-term coronal X-ray variability}

Further suggestive evidence for a connection between steady emission and coronal flares has come from the study of light curves. A strong correlation between $\mathrm{H} \gamma$ flare flux and simultaneous low-level X-ray flux in dMe stars suggests that a large number of flare-like events are always present (Butler et al. 1986). Continuous low-level variability due to flares has been frequently reported for active $\mathrm{M}$ dwarfs in particular, but also for earliertype dwarfs (Pollock et al. 1991; Vilhu et al. 1993; Kürster et al. 1997; Mathioudakis and Mullan 1999; Gagné et al. 1999). Evidence has also been reported for giants, including stars close to saturation (Haisch and Schmitt 1994; Ayres et al. 2001a; Fig. 25) and hybrid stars (Kashyap et al. 1994). Montmerle et al. (1983) estimated from light curves that 50\% of the observed X-ray emission in young stars in the $\rho$ Oph star-forming region is due to relatively strong flares. Maggio et al. (2000) found low-level variability in the very active AB Dor at a level of 20-25\% which they suggested to be due to ongoing low-level flaring (see similar conclusions by Stern et al. 1992b). When variability is studied for different plasma components, it is the hotter plasma that predominantly varies, while the cooler component is steady (Giampapa et al. 1996). An obvious suggestion is therefore that the high-temperature coronal component in active stars is the result of ongoing flaring.

\subsection{Stochastic variability - what is "quiescent emission"?}

The problem has been attacked in several dedicated statistical studies. While Ambruster et al. (1987) found significant continuous variability on time scales of several minutes in an Einstein sample of M dwarfs, Collura et al. (1988) concluded, from a similar investigation based on EXOSAT data, that the low-level episodes are truly "quiescent". Pallavicini et al. (1990a) found that approximately half of all investigated EXOSAT 
light curves show some residual variability, while the other half are constant. However, such statements can only be made within the limitations of the available sensitivity that also limits the ability to resolve fluctuations in time. Schmitt and Rosso (1988) and McGale et al. (1995) discussed and simulated in detail to what extent flares can be statistically detected in light curves as a function of the quiescent count rate, the relative flare amplitude, and the decay time. The essence is that a statistically significant number of counts must be collected during the finite time of a flare in order to define sufficient contrast against the "quiescent" background - otherwise the light curve is deemed constant.

Higher sensitivity became available with $R O S A T$, and the picture indeed began to change. Almost all ROSAT X-ray light curves of $\mathrm{M}$ stars are statistically variable on short ( $\lesssim 1$ day) time scales (Marino et al. 2000); this applies to a lesser extent to F-K dwarfs (Marino et al. 2003b). More specifically, the light curve luminosity distribution evaluated over time scales of hours to days is very similar to the equivalent distribution derived for solar flares, which suggests that the overall stellar light curves of dM stars are variable in the same way as a statistical sample of solar flares (Marino et al. 2000). Such variability may thus dominate flux level differences in snapshot observations taken several months apart (Kashyap and Drake 1999).

The EUVE satellite, while not being very sensitive, secured many observations from long monitoring programs that lasted up to 44 days. Some of these light curves reveal an astonishing level of continuous variability in main-sequence stars (Audard et al. 2000; Güdel et al. 2003a; Fig. 30), in RS CVn binaries (Osten and Brown 1999; Sanz-Forcada et al. 2002; Fig. 25), and in giants (Ayres et al. 2001a; Fig. 25). Some of those data were used to investigate statistical properties of the flare energy distribution (see Sect. 13.5).

With the advent of the much increased sensitivity offered by XMM-Newton and Chandra, weaker flares were uncovered, and they occur - expectedly - at higher rates. Güdel et al. (2002a, 2004) presented sensitive X-ray light curves of Proxima Centauri in which no time intervals longer than a few tens of minutes could be described as constant

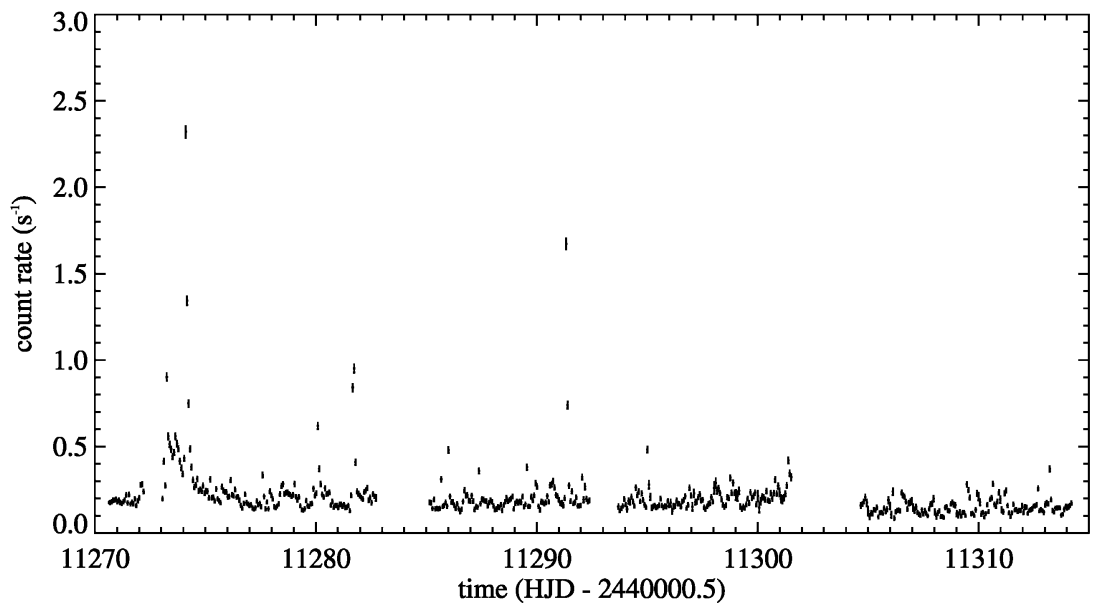

Fig. 30. A long light curve of the dMe star AD Leo, obtained by the DS instrument on EUVE. Most of the discernible variability is due to flares (after Güdel et al. 2003a) 


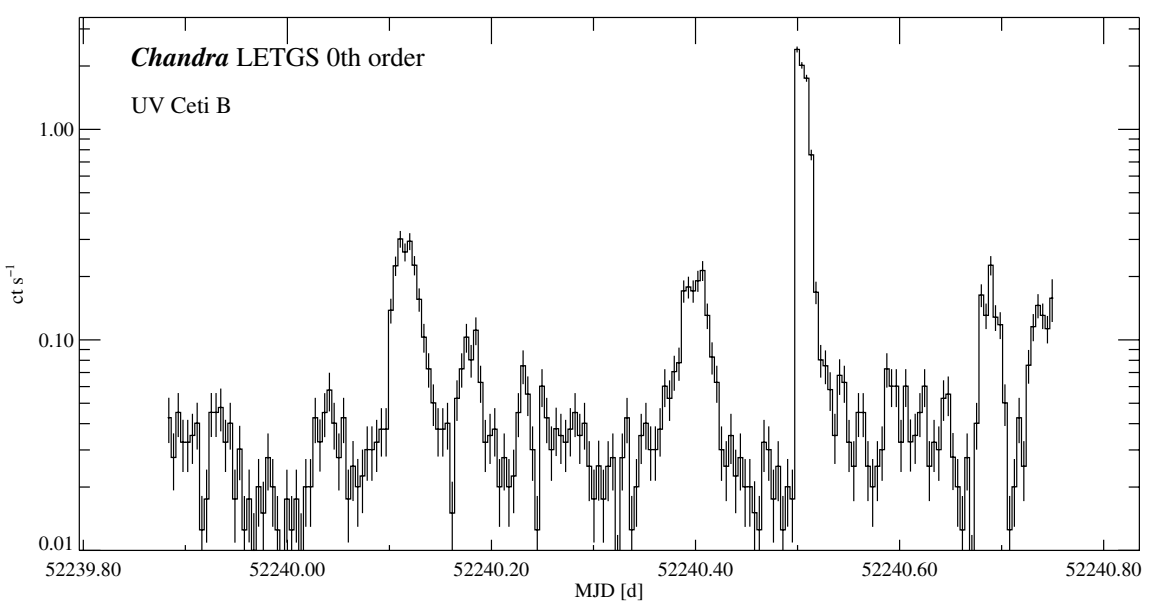

Fig. 31. Light curve of UV Ceti B, observed with the Chandra LETGS/HRC during about 1 day. Note the logarithmic flux axis (figure courtesy of M. Audard, after Audard et al. 2003b)

within the given sensitivity limit. Incidentally, this star was deemed constant outside obvious flares in the much less sensitive EXOSAT study by Pallavicini et al. (1990a). Frequent faint, flare-like X-ray fluctuations in this observation were often accompanied - in fact slightly preceded - by U band bursts, the latter being a signature of the initial bombardment of the chromosphere by high-energy electrons as discussed in Sect. 12.16. Audard et al. (2003b) estimated that no more than $30 \%$, and probably much less, of the long-term average X-ray emission of UV Cet can be attributed to any sort of steady emission, even outside obvious, large flares. On the contrary, almost the entire light curve is resolved into frequent, stochastically occurring flares of various amplitudes (Fig. 31). Many further observations from the new observatories reveal almost continual flaring (Stelzer et al. 2002; Stelzer and Burwitz 2003; van den Besselaar et al. 2003; Raassen et al. 2003b). For active binaries, Osten et al. (2002) found that the flux distributions of the low-level emission significantly deviate from the Poisson distributions expected from a constant source, once again pointing to continual variability.

\subsection{The solar analogy}

If variability is found in stellar light curves, its characteristic time scale is typically at least 3-5 minutes and often longer than 10 minutes (Ambruster et al. 1987; Pallavicini et al. 1990a; Güdel et al. 2002a). This motivated several authors to interpret low-level variability as being due to slow reconfigurations of active regions and emerging flux rather than due to stochastic flaring. The latter was expected to reveal itself in the form of short-term fluctuations, recalling the concept of "microflaring" in the solar corona.

There is, however, a widespread misconception that should briefly be discussed. A popular opinion has it that larger flares last longer, and that microvariability in stars should therefore express itself in short-term flickering. This view is not entirely correct. Statistical studies of solar flares usually do not find clear evidence for a dependence between flare duration and flare amplitude over quite wide a range in energy (Pearce and 
Harrison 1988; Feldman et al. 1997; Shimizu 1995). The distributions are dominated by the scatter in the duration, with durations ranging from 1 to 20 minutes even in the domain of quite small solar events. Aschwanden et al. (2000b) investigated scaling laws from solar nanoflares to large flares, covering 9 orders of magnitude in energy. They reported that the radiative and conductive time scales do not depend on the flare size. Güdel et al. (2003a) inspected the brightest flares in a long-duration EUV observation of AD Leo, again finding no trend. The latter authors also studied the sample of selected large flares from different stars reported by Pallavicini et al. (1990a); although a weak trend was found if four orders of magnitude in energy were included $\left(\tau \propto E^{0.25}\right)$, the scatter again dominated, and it is unclear what form of selection bias was introduced given that the sample consists only of well-detected bright flares (for the same reason, I refrain from performing statistics with the flares in Table 4; both the decay times and the total energies are subject to selection bias).

In fact, the claim that light curves are strictly bi-modal, separating detected flares from truly quiescent episodes, is tantamount to requiring that the peak flux of each flare must exceed a certain fraction of the "quiescent" emission level; since this level varies over at least 6 orders of magnitude in cool stars, the most luminous stars would be bound to produce only flares that exceed the largest solar events by many orders of magnitude, biased such that they are detected by available detectors. This view is not supported by solar observations: the solar flare rate increases steeply toward lower radiative energies, with no evidence (yet) for a lower threshold (e.g., Krucker and Benz 1998). Figure 32 shows an example of a GOES light curve in the 1.5-12 keV range, purposely selected during an extremely active period in November 2003. While the GOES band is harder than typical bands used for stellar observations, it more clearly reveals the level of the underlying variability (a typical detector used for stellar observations would see much less contrast). If the solar analogy has any merit in interpreting stellar coronal X-rays, then low-level emission in stars that do show flares cannot be truly quiescent, that is, constant or slowly varying exclusively due to long-term evolution of active regions, or due to rotational modulation. A measure of flare rates is therefore not meaningful unless

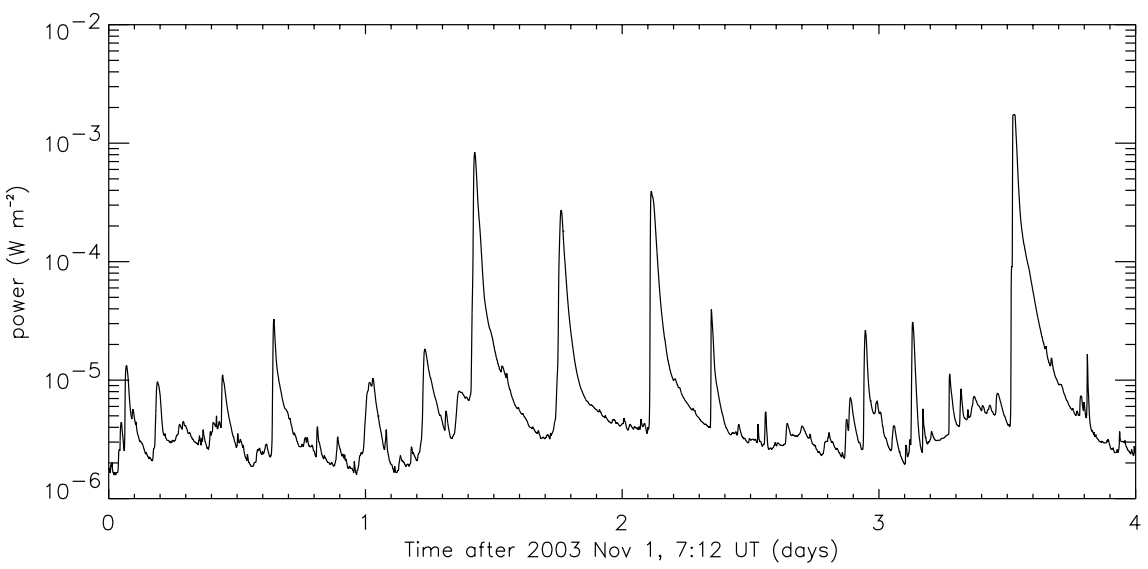

Fig. 32. GOES full-disk solar X-ray light curve, observed in the $1.5-12 \mathrm{keV}$ band in November 2003. The abscissa gives time after 2003 November 1, 7:12 UT in days 
it refers to flares above a given luminosity or energy threshold. This is - emphatically not to say that steady emission is absent. However, once we accept the solar analogy as a working principle, the question is not so much about the presence of large numbers of flares, but to what extent they contribute to the overall X-ray emission from coronae.

\subsection{The flare-energy distribution}

The suggestion that stochastically occurring flares may be largely responsible for coronal heating is known as the "microflare" or "nanoflare" hypothesis in solar physics (Parker 1988). Observationally, it is supported by evidence for the presence of numerous smallscale flare events occurring in the solar corona at any time (e.g., Lin et al. 1984). Their distribution in energy is a power law,

$$
\frac{d N}{d E}=k E^{-\alpha}
$$

where $d N$ is the number of flares per unit time with a total energy in the interval $[E, E+d E]$, and $k$ is a constant. If $\alpha \geq 2$, then the energy integration (for a given time interval) diverges for $E_{\min } \rightarrow 0$, that is, by extrapolating the power law to sufficiently small flare energies, any energy release power can be attained. This is not the case for $\alpha<2$. Solar studies have repeatedly resulted in $\alpha$ values of $1.6-1.8$ for ordinary solar flares (Crosby et al. 1993), but some recent studies of low-level flaring suggest $\alpha=2.0-2.6$ (Krucker and Benz 1998; Parnell and Jupp 2000).

Relevant stellar studies have been rare (see Table 5). Early investigations lumped several stars together to produce meaningful statistics. A set of $\mathrm{M}$ dwarf flares observed with EXOSAT resulted in $\alpha \approx 1.5$ (Collura et al. 1988), and similarly, the comprehensive study by Pallavicini et al. (1990a) of an EXOSAT survey of M dwarfs implied $\alpha \approx 1.7$. Osten and Brown (1999) used EUVE data to perform a similar investigation of flares on RS CVn-type binaries, and again by lumping stellar samples together they inferred $\alpha \approx 1.6$.

However, several biases may affect statistical flare studies, all related to the illdetermined problem of flare identification in stellar observations. First, detecting flares

Table 5. Stellar radiative flare-energy distributions

\begin{tabular}{lllll}
\hline Star sample & $\begin{array}{l}\text { Photon energies } \\
{[\mathrm{keV}]}\end{array}$ & $\begin{array}{l}\log (\text { Flare } \\
\text { energies })^{\mathrm{a}}\end{array}$ & $\alpha$ & References \\
\hline M dwarfs & $0.05-2$ & $30.6-33.2$ & $1.52 \pm 0.08$ & Collura et al. (1988) \\
M dwarfs & $0.05-2$ & $30.5-34.0$ & $1.7 \pm 0.1$ & Pallavicini et al. (1990a) \\
RS CVn binaries & EUV & $32.9-34.6$ & 1.6 & Osten and Brown (1999) \\
Two G dwarfs & EUV & $33.5-34.8$ & $2.0-2.2$ & Audard et al. (1999) \\
F-M dwarfs & EUV & $30.6-35.0$ & $1.8-2.3$ & Audard et al. (2000) \\
Three M dwarfs & EUV & $29.0-33.7$ & $2.2-2.7$ & Kashyap et al. (2002) \\
AD Leo & EUV and 0.1-10 & $31.1-33.7$ & $2.0-2.5$ & Güdel et al. (2003a) \\
AD Leo & EUV & $31.1-33.7$ & $2.3 \pm 0.1$ & Arzner and Güdel (2004) \\
\hline
\end{tabular}

\footnotetext{
${ }^{\mathrm{a}}$ Total flare-radiated X-ray energies used for the analysis (in ergs).
} 

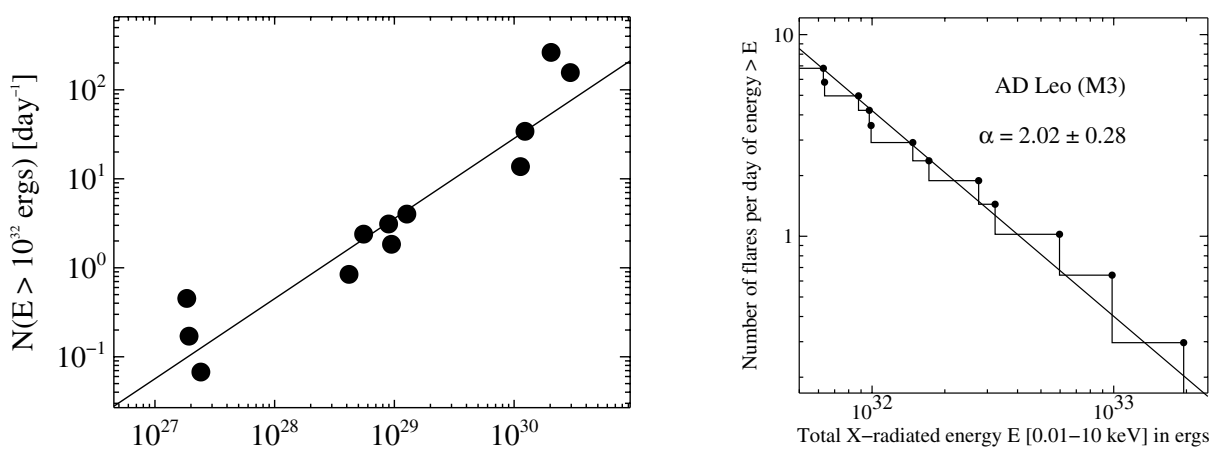

Fig. 33. Left: The rate of flares above a threshold of $10^{32} \mathrm{erg}$ in total radiated X-ray energy is plotted against the low-level luminosity for several stars, together with a regression fit. Right: Flare energy distribution for AD Leo, using a flare identification algorithm for an observation with EUVE (both figures courtesy of M. Audard, after Audard et al. 2000)

in light curves is a problem of contrast. Poisson counting statistics increases the detection threshold for flares on top of a higher continuous emission level. The low-energy end of the distribution of detected flares is therefore ill-defined and is underrepresented. Further, large flares can inhibit the detection of the more numerous weak flares during an appreciable fraction of the observing time. And lastly, the detection threshold also depends on stellar distance; by lumping stars together, the low-energy end of the distribution becomes invariably too shallow.

To avoid biases of this kind, Audard et al. (1999) and Audard et al. (2000) applied a flare search algorithm to EUVE light curves of individual active main-sequence stars, taking into account flare superpositions and various binning to recognize weak flares, and performing the analysis on individual light curves. Their results indicate a predominance of relatively steep power laws including $\alpha \geq 2$ (an example is shown in Fig. 33b).

Full forward modeling of a superposition of stochastic flares was applied to EUV and X-ray light curves by Kashyap et al. (2002) and Güdel et al. (2003a) based on Monte Carlo simulations, and by Arzner and Güdel (2004) based on an analytical formulation. ${ }^{7}$ The results of these investigations are in full agreement, converging to $\alpha \approx 2.0-2.5$ for $\mathrm{M}$ dwarfs (Table 5). If the power-law flare energy distribution extends by about 1-2 orders of magnitude below the actual detection limit in the light curves, then the entire emission could be explained by stochastic flares. The coronal heating process in magnetically active stars would - in this extreme limit - be one solely due to timedependent heating by flares, or, in other words, the X-ray corona would be an entirely hydrodynamic phenomenon rather than an ensemble of hydrostatic loops.

7 Note that the distribution of measured fluxes does not describe the flare amplitude distribution. The problem of inverting the former to obtain the latter was analytically solved by Arzner and Güdel (2004). 


\subsection{Observables of stochastic flaring}

In order for stochastic flaring to be an acceptable coronal heating mechanism, it should explain a number of X-ray observables. Flares develop characteristically in EM and $T$ (fast rise to peak, slow decay). Therefore, the superposition of a statistical ensemble of flares produces a characteristic time-averaged DEM. For simple flare decay laws, the resulting DEM is analytically given by (14) that fits observed, steeply rising DEMs excellently (Güdel et al. 2003a). An extension of the Kopp and Poletto (1993) formalism can be used numerically to find approximations of a DEM that results by time-averaging the $\operatorname{EM}(T, t)$ of a population of cooling, stochastic flares drawn from a distribution with a prescribed $\alpha$; these DEMs show two characteristic maxima, the cooler one being induced by the large rate of small flares, and the hotter one being due to the few large flares with high temperature and EM, somewhat similar to double-peaked DEMs that were derived from observations; in this interpretation, the higher rate of larger flares in more active stars shifts the EM to higher temperatures, as has been found in evolutionary sequences of solar analogs (Güdel et al. 1997a; Güdel 1997; Skinner and Walter 1998).

As for the light curves, the superposition of stochastically occurring flares produces an apparently steady baseline emission level that is constantly present and that may be mistaken for a truly quiescent component (Kopp and Poletto 1993), in particular for large $\alpha$ values in (60). Figure 34 shows simulations of superimposed flares drawn from
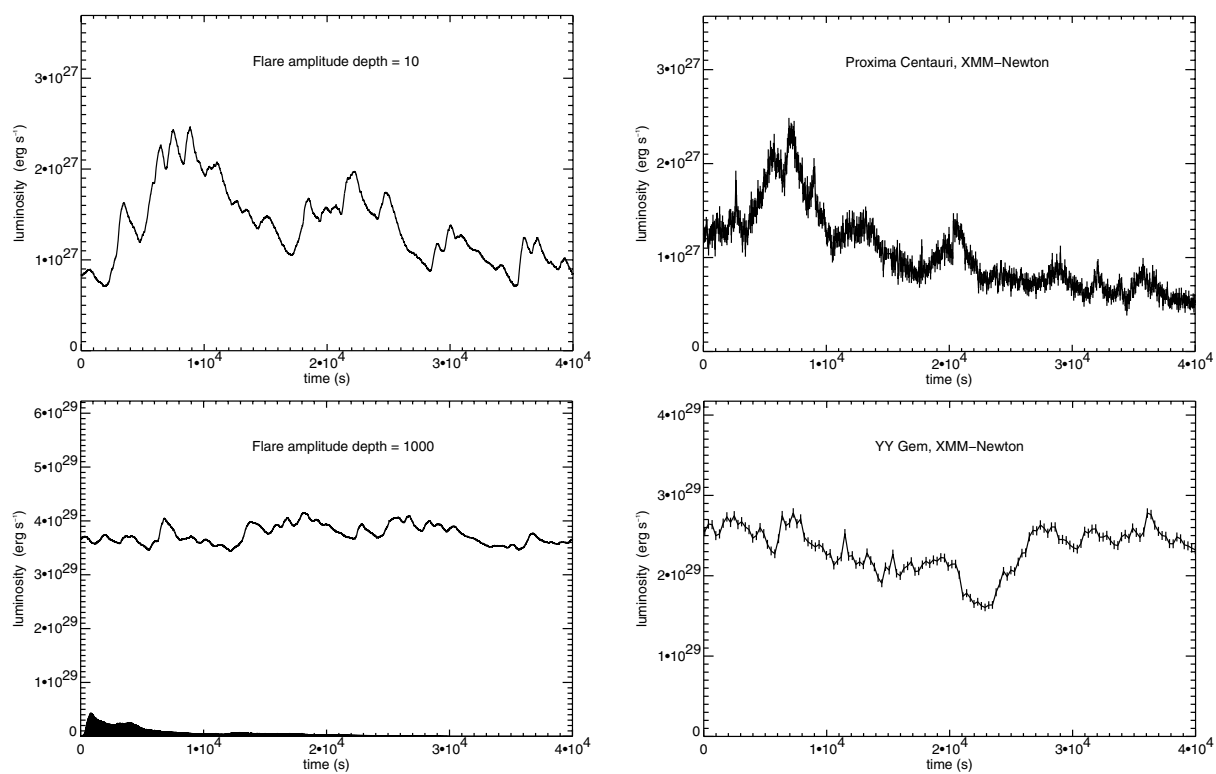

Fig. 34. X-ray light curves. Left panels: Synthetic light curves from superimposed flares; largest to smallest amplitude $=10$ (upper) and 1000 (lower plot). No truly quiescent emission has been added. A giant flare on Proxima Centauri (Güdel et al. 2002a) has been used as a shape template, shown filled in the lower plot for the maximum amplitude contributing to the light curve. Right panels: Observed X-ray light curves for comparison. Proxima Centauri (upper plot, Güdel et al. 2004) and YY Gem (lower plot, including eclipse; after Güdel et al. 2001a) 
a power-law distribution with $\alpha=2.2$, compared with observations. No truly steady emission has been added. The large flare observed on Proxima Centauri (Güdel et al. 2004) was used as a shape template from which all flares in the simulation were scaled. The first example uses flare energies spread over a factor of ten only, the second uses three orders of magnitude. In the latter case, the light curve has smoothed out to an extent that it is dominated by "quiescent" emission. This effect is stronger for larger $\alpha$. The individual peaks are merely the peaks of the most energetic flares in the ensemble. For small (dM) stars, the available stellar area may in fact constrain the energy range of stochastic flares because flaring active regions may cover a significant fraction of the surface, which limits the number of simultaneous flares (see upper example in Fig. 34, e.g., Proxima Centauri) and thus makes lower-mass stars statistically more variable.

Finally, spectroscopic density measurements of a time-integrated, stochastically flaring corona should yield values equivalent to the densities derived from time-integrating the spectrum of a large flare. There is suggestive evidence in support of this. While large stellar X-ray flares achieve peak electron densities of several times $10^{11} \mathrm{~cm}^{-3}$, the time-integrated X-ray spectrum of the Proxima Centauri flare described by Güdel et al. (2002a, 2004) (Fig. 28) yields a characteristic density of $\log n_{e} \approx 10.5 \pm 0.25$ derived from O vII triplet, which compares favorably with densities in magnetically active MS stars during low-level emission (Ness et al. 2001, 2002a, Sect. 10.2).

\section{X-ray absorption features and prominences}

X-ray spectra are sensitive to photoelectric absorption by cooler foreground gas. The absorption column is a powerful diagnostic for the amount of cool circumstellar gas although little can be said about its distribution along the line of sight. X-ray attenuation by the interstellar medium is generally weak for field stars within a few $100 \mathrm{pc}$, but it becomes very prominent for deeply embedded stars in star formation regions, or stars that are surrounded by thick accretion disks.

However, anomalous absorption is sometimes also recorded in more solar-like, nearby stars, in particular during large flares. The observed column densities may vary by typically a factor of two on short time scales. Examples were presented by Haisch et al. (1983) for Proxima Cen, Ottmann and Schmitt (1996) for Algol, and by Ottmann and Schmitt (1994) for AR Lac. Much larger absorption column densities have occasionally been measured, up to $4 \times 10^{22} \mathrm{~cm}^{-2}$ in a flare on V733 Tau (Tsuboi et al. 1998) and $3 \times 10^{21} \mathrm{~cm}^{-2}$ in the course of a large flare on Algol (Favata and Schmitt 1999). The cause of this anomalous absorption is not clear. From the solar analogy, it is possible that prominences related with the flare region pass in front of the X-ray source and temporarily shadow part of it. Coronal mass ejections sometimes accompany solar flares. If the material cools sufficiently rapidly by radiation and expansion, it may also attenuate the X-rays. The detection of ejected mass is of some relevance in binaries. In systems like Algol, the mass may flow onto the binary companion or form a temporary accretion disk around it (Stern et al. 1992a). This could explain why excess absorption has been found also during low-level emission episodes in several Algol-type binaries (Singh et al. 1995). In rapidly rotating single stars, increased amounts of hydrogen may condense out of large cooling loops that grow unstable near their apexes, a suggestion that has 
found direct support in optical observations of AB Dor (e.g., Collier Cameron 1988; Donati 1999).

\section{Resonance scattering and the optical depth of stellar coronae}

When studies of the initial spectra from the EUVE satellite encountered unexpectedly low line-to-continuum ratios (e.g., Mewe et al. 1995), line suppression by optical depth effects due to resonant scattering in stellar coronae surfaced as one possible explanation (Schrijver et al. 1994). Although we nowadays attribute these anomalies mostly to abundance anomalies and to an excess "pseudo"-continuum from weak lines, a search for non-zero optical depths in coronae is worthwhile because it may be used as another tool to study coronal structure. Non-negligible optical depths would also affect our interpretation of stellar coronal X-ray spectra for which we usually assume that the corona is entirely optically thin.

Resonant scattering requires optical depths in the line centers of $\tau \gtrsim 1$. The latter is essentially proportional to $n_{e} \ell / T^{1 / 2}$ (Mewe et al. 1995). For static coronal loops, this implies $\tau \propto T^{3 / 2}$ (Schrijver et al. 1994; e.g., along a loop or for a sample of nested loops in a coronal volume). Numerical values for $\tau$ are given in Schrijver et al. (1994) and Mewe et al. (1995) for several EUV lines. If the optical depth in a line is significant in a coronal environment, then the absorbed photon will be re-emitted ("scattered") prior to collisional de-excitation. Continuum photons, on the other hand, are much less likely to be scattered. The effects of resonant line scattering into and out of the line of sight, however, cancel in a homogeneous source, but the stellar surface that absorbs down-going photons breaks the symmetry: The line-to-continuum ratio is reduced if the emitting volume is smaller than the scattering volume and lies closer to the stellar surface (Schrijver et al. 1994; Mewe et al. 1995). This situation is fulfilled in a corona since the scattering efficiency decreases only with $n_{e}$ while the emissivity decreases more rapidly, $\propto n_{e}^{2}$. Schrijver et al. (1994) predicted suppression of the line fluxes by up to a factor of two in the most extreme cases.

A number of applications to EUVE spectra from stars at different activity levels have been given by Schrijver et al. (1995). The authors concluded that significant optical depths may be present in particular in inactive stars such as $\alpha$ Cen and Procyon. The scattering layer would most probably be an extended hot envelope or a stellar wind. This interpretation was subsequently challenged, however, by Schmitt et al. (1996a) in a detailed study of the EUV continuum and a comparison of flux levels in the ROSAT spectral range. They attributed the anomalously low line-to-continuum ratios to an excess EUV continuum that builds up from the superposition of many weak lines that are not tabulated in present-day codes.

In the X-ray range, the usually strong Ne-like Fe XVII lines provide excellent diagnostics. The Fe XVII $\lambda 15.01$ line is often chosen for its large oscillator strength. Its flux is compared with the fluxes of Fe XVII lines with low oscillator strengths such as those at $15.26 \AA$ and at $16.78 \AA$. There is considerable uncertainty in the atomic physics, however, that has equally affected interpretation of solar data. While Schmelz et al. (1997) and Saba et al. (1999) found evidence for optical depth effects in the Fe XVII $\lambda 15.01$ line, recent laboratory measurements of the corresponding flux ratios differ significantly from 
previous theoretical calculations (Brown et al. 1998, 2001; Laming et al. 2000). New calculations have recently been presented by, for example, Doron and Behar (2002).

Stellar coronal optical depths have become accessible in the X-ray range with Chan$d r a$ and XMM-Newton. No significant optical depth effects were found for Fe XVII $\lambda 15.01$ for a restricted initial sample of stars such as Capella and Procyon (Brinkman et al. 2000; Ness et al. 2001; Mewe et al. 2001; Phillips et al. 2001). Ness et al. (2001) calculated upper limits to optical depths based on measured EM and electron densities, assuming homogeneous sources. They found no significant optical depths although none of these stars is very active. Huenemoerder et al. (2001), Ness et al. (2002b) and Audard et al. (2003a) extended the Fe XVII diagnostics to the high end of magnetic activity, viz. II Peg, Algol and a sample of RS CVn binaries, respectively, but again reported no evidence for optical depth effects. The problem was comprehensively studied by Ness et al. (2003a) in a survey of 26 stellar coronae observed with XMM-Newton and Chandra across all levels of activity. They again used the Fe XVII $\lambda 15.27 / \lambda 15.01$ and $\lambda 16.78 / \lambda 15.01$ diagnostics as well as the ratios between the resonance and the forbidden lines in He-like line triplets of $\mathrm{O}$ VII and Ne IX. Many line ratios are at variance with solar measurements and with calculated predictions, but the latter themselves are uncertain (see above). The interesting point is, however, that the flux ratios are similar for all stars except for those with the very coolest coronae for which line blends are suspected to bias the flux measurements. Ness et al. (2003a) concluded that optical depth effects are absent on all stars at least in the relevant temperature regime, rather than requiring non-zero but identical optical depth in such variety of stellar coronae. These conclusions also extend to line ratios in Ly $\alpha$ series (e.g., Ly $\alpha: \operatorname{Ly} \beta$ for O vIII, Nex, or Si XIV, Huenemoerder et al. 2001; Osten et al. 2003).

Related effects were previously considered, but also questioned, for the Fe K complex at 6.7-7 keV in intermediate-resolution observations (Tsuru et al. 1989; Stern et al. 1992a; Singh et al. 1996a). Lastly, Güdel et al. (2004) measured Fe XVII flux ratios during a strong flare on Proxima Centauri but again found neither discrepant values nor a time evolution that would contradict an optically thin assumption.

To conclude, then, it appears that no X-ray optical depth effects have unambiguously been detected in any stellar corona investigated so far, notwithstanding the large range of geometries, temperatures, and densities likely to be involved in stars across the spectrum of activity, including large flares. Further checks of individual cases or of large flares remain worthwhile, however, given the potential diagnostic power of resonant scattering effects. $^{8}$

\section{The elemental composition of stellar coronae}

It is quite well established that the solar corona and the solar wind show an elemental composition at variance with the composition of the solar photosphere. Whatever the reason for the discrepancy, our interest in understanding element abundances in stellar coronae is twofold: Observationally, because they shape the X-ray spectra from which we

\footnotetext{
${ }^{8}$ Note added in proof: A recent report by Testa et al. (2004, ApJ, 609, L79) indicates evidence of resonance scattering in $\mathrm{Ly} \alpha / \mathrm{Ly} \beta$ line-flux ratios of $\mathrm{O}$ VIII and $\mathrm{Nex}$ in the RS CVn binaries II Peg and IM Peg. The inferred path lengths vary between $2 \times 10^{-4} R_{*}$ and $4 \times 10^{-2} R_{*}$.
} 
derive basic coronal parameters; and physically, because abundance anomalies reflect diffusion processes and element fractionation mechanisms in the chromosphere and transition region, and possibly the physics of mass transport into the corona.

\subsection{Solar coronal abundances: A brief summary}

Measurements of the composition of the solar corona have revealed what is commonly known as the "First Ionization Potential (FIP) Effect": Essentially, elements with a FIP above $\approx 10 \mathrm{eV}$ (e.g., C, N, O, Ne, Ar) show photospheric abundance ratios with respect to hydrogen, while elements with a smaller FIP (e.g., $\mathrm{Si}, \mathrm{Mg}, \mathrm{Ca}, \mathrm{Fe}$ ) are overabundant by a factor of a few. This picture was comprehensively summarized in the extensive work by Meyer (1985a,b) who showed that the same FIP effect is also present in the solar wind, in solar energetic particles, and - quite surprisingly - in cosmic rays. ${ }^{9}$ The latter finding immediately suggested that the seeds of cosmic rays may perhaps be ejected by active stellar coronae that are subject to a similar, solar-like abundance anomaly. An extension of the currently accepted picture was given by Feldman (1992) who discussed abundance anomalies in various solar features, pointing out that the degree of the FIP anomaly varies from feature to feature.

I refer the interested stellar reader to the extensive solar literature on the solar FIP effect, and in particular to a review of solar FIP models by Hénoux (1995), overviews presented by Jordan et al. (1998) and in the papers by Drake et al. (1995b) and Laming et al. (1995). I only briefly summarize a few principal points. Current thinking is that a fractionation process, probably involving electric and/or magnetic fields or pressure gradients, occurs at chromospheric levels where low-FIP elements are predominantly ionized and high-FIP elements are predominantly neutral. Ions and neutrals are then affected differently by electric and magnetic fields. A successful model for the FIP effect will eventually have to explain why and how low-FIP elements are transported into the corona at an enhanced rate.

The FIP effect is most pronounced in relatively evolved solar coronal features such as old loops, but also in magnetically open regions. In contrast, young, compact active regions and newly emerged structures reveal photospheric composition. The latter mixture is also most evident in flares, which suggests that new material is brought up from photospheric/chromospheric layers that has not been subject to fractionation. Notable exceptions exist, such as Ca-rich flares (Sylwester et al. 1984), and Ne-rich flares (Schmelz 1993).

In the light of the diverse FIP anomalies in the solar corona, stellar observations should obviously be compared with Sun-as-a-star data. To this end, Laming et al. (1995) have studied the FIP anomaly by making use of full-disk solar spectra. While they confirmed the presence of an overall coronal enrichment of low-FIP elements by factors of about 3-4, they somewhat surprisingly reported an absence of a FIP bias at subcoronal temperatures $(<1 \mathrm{MK})$. They suggested that the strength of the FIP effect is in fact

\footnotetext{
${ }^{9}$ I note in passing that Meyer normalized the abundances such that the low-FIP element abundances were photospheric and the high-FIP abundances depleted, whereas present-day wisdom has the high-FIP elements at photospheric levels, and the low-FIP elements enriched in the corona (Feldman 1992).
} 
a function of altitude, with the lower-temperature emission being dominated by the supergranulation network that shows photospheric abundances.

\subsection{Stellar coronal abundances: The pre-XMM-Newton/Chandra view}

Early X-ray observatories typically lacked the spectral resolution required to make solid statements about coronal abundances. Some indications of possible depletions of Fe were reported during very strong flares, as will be summarized in Sect. 16.6. Also, some early medium-resolution spectra apparently required "anomalous abundances" for a successful model fit (Walter et al. 1978b; Swank et al. 1981). Despite these initial developments, element abundances became a non-issue as the available detectors simply did not permit their unambiguous determination. It was customary to adopt a coronal composition equal to the solar photospheric mixture. The basis for this assumption is that most stars in the solar neighborhood belong to Population I, and for these stars we expect a near-solar composition, even if the Sun is a somewhat metal-rich star. On the other hand, the known FIP bias of the solar corona should be more of a concern when interpreting stellar spectra, but the large variations in the solar coronal composition would have made any "standard coronal abundance" tabulation quite arbitrary. As we now know, adopting such a coronal standard would have been useless.

When ASCA introduced routine medium-resolution X-ray spectroscopy based on CCD technology with a resolving power of $R \approx 10-30$, and $E U V E$ allowed for wellresolved EUV line spectroscopy with $R \approx 300$, some classes of magnetically active stars started to show perplexing abundance features that were neither comparable with those of the solar corona nor compatible with any pattern expected from the photospheric composition (which is often not well determined either).

The only way to reconcile thermal models with the observed CCD spectra was to introduce depleted abundances in particular of $\mathrm{Mg}, \mathrm{Si}$, and $\mathrm{Fe}$ but also of other elements (White et al. 1994; N. White 1996; Antunes et al. 1994; Gotthelf et al. 1994; S. Drake et al. 1994a; S. Drake 1996; Singh et al. 1995, 1996a; Mewe et al. 1996; Kaastra et al. 1996). Abundances of $\mathrm{Fe}$ as low as $10-30 \%$ of the solar photospheric value were regularly reported. Little in the way of systematic trends was present for the various other elements (S. Drake 1996; Kaastra et al. 1996). Some observations with ASCA indicated the presence of a "relative" FIP effect because the high-FIP elements were more depleted than the low-FIP elements while all abundances were subsolar, but the evidence was marginal and was not followed by Fe (S. Drake et al. 1994a; Tagliaferri et al. 1997; Güdel et al. 1997a for Mg). Generally, metal depletion was found to be strongest in the most active stars (Singh et al. 1995, 1999; S. Drake 1996).

EUVE confirmed the considerable metal depletion for magnetically active stars based on unexpectedly low Fe line-to-continuum ratios (Stern et al. 1995a). Extreme cases such as CF Tuc showed almost no Fe lines, requiring Fe abundances as low as $10 \%$ solar (Schmitt et al. 1996d). Further examples of significant metal depletion were reported by Rucinski et al. (1995) and Mewe et al. (1997).

EUVE spectra, however, also revealed a solar-like FIP-related bias, but this was found exclusively for weakly or intermediately active stars such as $\alpha$ Cen (Drake et al. 1997), $\epsilon$ Eri (Laming et al. 1996) and $\xi$ Boo A (Laming and Drake 1999; Drake and Kashyap 2001). Mewe et al. (1998a) found further indications for a FIP effect in $\alpha$ Cen also from 
ASCA observations, but no magnetically active stars were ever reported with a similar coronal composition. Cosmic rays thus no longer appeared to be related to active stars as far as the abundance mix was concerned (Drake et al. 1995b).

A peculiarity was found for the inactive Procyon, namely identical coronal and photospheric compositions (Drake et al. 1995a,b). A possible cause of this anomaly among anomalies is that the supergranulation network, assumed to be of photospheric composition as in the solar case, reaches to higher temperatures.

As discussed in Sect. 9.3, the X-ray spectroscopic abundance determination is strongly tangled with the derivation of the DEM, and the caveats and debates described there apply here. The problem is particularly serious in low-resolution X-ray spectra, as discussed by Singh et al. (1999) and Favata et al. (1997a). Despite a decent capability of CCD detectors to recover temperatures and EMs, some caveats apply to the abundance determination in particular if the considered spectral ranges are too restricted. The state of the field remained unsatisfactory, and the lack of systematics made much of the physical interpretation quite ambiguous.

\subsection{Stellar coronal abundances: New developments with XMM-Newton and Chandra}

At least some clarification came with the advent of high-resolution X-ray grating spectroscopy. Early observations of HR 1099 and AB Dor with the XMM-Newton Reflection Grating Spectrometer uncovered a new, systematic FIP-related bias in magnetically active stars: in contrast to the solar case, low-FIP abundances are systematically depleted with respect to high-FIP elements (Brinkman et al. 2001; Güdel et al. 2001b; Audard et al. 2001a; Fig. 35), a trend that has been coined the "inverse FIP effect" (IFIP). As a consequence of this anomaly, the ratio between the abundances of $\mathrm{Ne}$ (highest FIP) and $\mathrm{Fe}$ (low FIP) is unusually large, of order 10, compared to solar photospheric conditions.

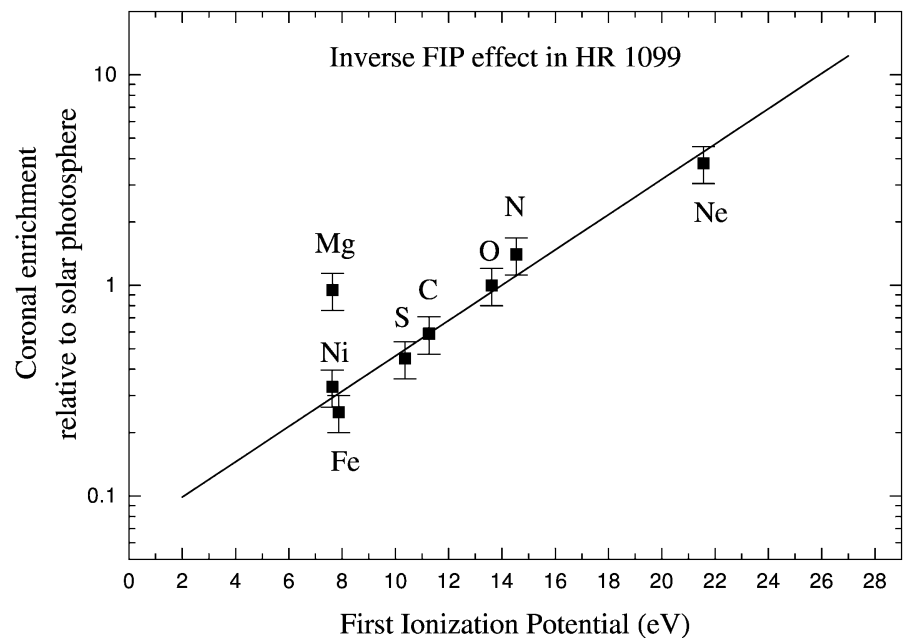

Fig. 35. Inverse FIP effect in the corona of HR 1099. The coronal element abundance ratios with respect to oxygen and normalized to the solar photospheric ratios are plotted as a function of the FIP of the respective element (after Brinkman et al. 2001) 
These trends have been widely confirmed for many active stars and from the various gratings available on XMM-Newton or Chandra (e.g., Drake et al. 2001; Huenemoerder et al. 2001, 2003; Raassen et al. 2003b; van den Besselaar et al. 2003, to name a few). With respect to the hydrogen abundance, most elements in active stars remain, however, depleted (Güdel et al. 2001a,b; Audard et al. 2001a), and this agrees with the overall findings reported previously from low-resolution spectroscopy. Strong Ne enhancements can be seen, in retrospect, also in many low-resolution data discussed in the earlier literature, and the IFIP trend has now also been traced into the pre-main sequence domain by Imanishi et al. (2002).

When stellar spectra covering a wide range of magnetic activity are compared, only highly active stars show the presence of an IFIP pattern. In intermediately active stars, flat abundance distributions are recovered (Audard et al. 2003a). The abundances revert to a normal, solar-type FIP anomaly for stars at activity levels of $\log L_{X} / L_{\text {bol }} \lesssim-4$ (Güdel et al. 2002c; Telleschi et al. 2004, Fig. 36). Whenever the IFIP pattern is present, all abundances appear to be sub-solar, but the $\mathrm{Fe} / \mathrm{H}$ abundance ratio gradually rises with decreasing coronal activity. The transition from an IFIP to a solar-like FIP abundance pattern and from very low Fe abundances to mild depletion seems to coincide with i) the transition from coronae with a prominent hot $(T \gtrsim 10 \mathrm{MK})$ component to cooler coronae, and ii) with the transition from prominent non-thermal radio emission to the absence thereof (Güdel et al. 2002c).

In order to illustrate the above trends more comprehensively, Table 6 summarizes a few important parameters from recent abundance determinations based on high-resolution spectroscopy. The table gives absolute Fe abundances, ratios of the high-FIP elements $\mathrm{O}$ and $\mathrm{Ne}$ with respect to $\mathrm{Fe}$, and the ratios between the two low-FIP element abundances of $\mathrm{Mg}$ and $\mathrm{Fe}$ and between the two high-FIP element abundances of $\mathrm{Ne}$ and O. Direct comparison of reported abundances should generally be treated with caution because various solar photospheric standards have been adopted. As far as possible, I
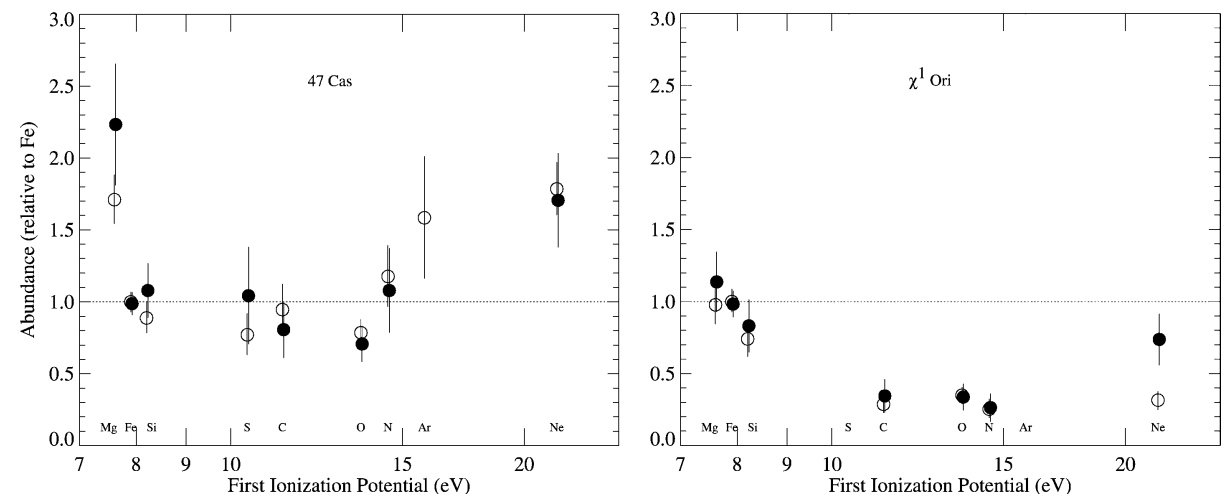

Fig. 36. Coronal abundance determination for solar analogs. Left: 47 Cas, a very active nearZAMS star; right: $\chi^{1}$ Ori, an intermediately active solar analog. Abundances are given relative to Fe, and refer to solar photospheric abundances as given by Anders and Grevesse (1989) and Grevesse and Sauval (1999). Filled circles refer to determinations that used selected line fluxes of Fe for the DEM reconstruction; open circles show values found from a full spectral fit (figures courtesy of A. Telleschi, after Telleschi et al. 2004) 
Table 6. Element abundances ${ }^{\mathrm{a}}$ from high-resolution spectroscopy

\begin{tabular}{|c|c|c|c|c|c|c|c|}
\hline Star & $\mathrm{I}^{\mathrm{b}}$ & $\bar{T}$ & $\mathrm{Fe}$ & $\mathrm{Ne} / \mathrm{Fe}$ & $\mathrm{O} / \mathrm{Fe}$ & $\mathrm{Mg} / \mathrm{Fe}$ & Reference \\
\hline Procyon & $\mathrm{L}$ & 1.4 & 0.66 & 1.5 & 1.0 & 1.1 & Raassen et al. (2002) \\
\hline Procyon & $\mathrm{L}$ & 1.45 & 0.98 & 1.08 & 0.37 & 1.66 & Sanz-Forcada et al. (2004) \\
\hline Procyon & $\mathrm{R}$ & 1.8 & 1.1 & 1.04 & 0.68 & $\ldots$ & Raassen et al. (2002) \\
\hline$\alpha$ Cen A & $\mathrm{L}$ & 1.5 & 1.36 & 0.37 & 0.3 & 1.01 & Raassen et al. (2003a) \\
\hline$\alpha$ Cen B & $\mathrm{L}$ & 1.8 & 1.43 & 0.38 & 0.23 & 1.12 & Raassen et al. (2003a) \\
\hline Prox Cen & $\mathrm{R}$ & 3.7 & 0.51 & 1.6 & 0.6 & 2.1 & Güdel et al. (2004) \\
\hline$\epsilon$ Eri & $\mathrm{L}$ & 4.0 & 0.74 & 1.35 & 0.53 & 0.95 & Sanz-Forcada et al. (2004) \\
\hline$\chi^{1}$ Ori & $\mathrm{R}$ & 4.4 & 0.87 & 0.73 & 0.33 & 1.12 & Telleschi et al. $(2004)^{\mathrm{c}}$ \\
\hline$\kappa^{1}$ Cet & $\mathrm{R}$ & 4.5 & 1.18 & 0.95 & 0.39 & 1.94 & Telleschi et al. $(2004)^{c}$ \\
\hline$\pi^{1} \mathrm{UMa}$ & $\mathrm{R}$ & 4.5 & 0.81 & 0.62 & 0.32 & 1.24 & Telleschi et al. $(2004)^{c}$ \\
\hline AD Leo & $\mathrm{R}$ & 5.8 & 0.34 & 2.5 & 1.21 & 1.13 & van den Besselaar et al. (2003) \\
\hline AD Leo & $\mathrm{L}$ & 6.1 & 0.39 & 3.43 & 1.64 & 0.6 & van den Besselaar et al. (2003) \\
\hline Capella & $\mathrm{R}$ & 5.0 & 0.92 & 0.64 & 0.32 & 1.22 & Audard et al. (2003a) \\
\hline Capella & $\mathrm{R}$ & 6.5 & 0.84 & 0.50 & 0.50 & 1.08 & Audard et al. (2001b) \\
\hline Capella & $\mathrm{L}$ & 6.5 & 1.0 & 0.5 & 0.48 & 0.91 & Argiroffi et al. (2003) \\
\hline YY Gem & $\mathrm{R}$ & 7.6 & 0.21 & 3.62 & 1.42 & 0.81 & Güdel et al. (2001a) \\
\hline$\sigma^{2} \mathrm{CrB}$ & $\mathrm{H}$ & 9 & 0.46 & 1.40 & 0.55 & 0.99 & Osten et al. (2003) \\
\hline EK Dra & $\mathrm{R}$ & 9.1 & 0.72 & 1.01 & 0.51 & 1.54 & Telleschi et al. $(2004)^{\mathrm{c}}$ \\
\hline AT Mic & $\mathrm{R}$ & 9.2 & 0.30 & 4.8 & 3.2 & 1.4 & Raassen et al. (2003b) \\
\hline $47 \mathrm{Cas}$ & $\mathrm{R}$ & 10.6 & 0.50 & 1.68 & 0.70 & 2.21 & Telleschi et al. $(2004)^{c}$ \\
\hline AB Dor & $\mathrm{R}$ & 10.0 & 0.40 & 4.8 & 2.23 & 0.95 & Sanz-Forcada et al. (2003) \\
\hline AB Dor & $\mathrm{R}$ & 11.4 & 0.33 & 3.04 & 1.22 & 0.83 & Güdel et al. (2001b) \\
\hline V851 Cen & $\mathrm{R}$ & 11 & 0.56 & 5.5 & 1.76 & 1.6 & Sanz-Forcada et al. (2004) \\
\hline$\lambda$ And & $\mathrm{H}$ & 11 & 0.37 & 2.23 & 1.35 & 1.56 & Sanz-Forcada et al. (2004) \\
\hline$\lambda$ And & $\mathrm{R}$ & 13.2 & 0.2 & 5.3 & 1.75 & 2.95 & Audard et al. (2003a) \\
\hline VY Ari & $\mathrm{R}$ & 11.3 & 0.18 & 7.0 & 2.2 & 1.83 & Audard et al. (2003a) \\
\hline Algol & $\mathrm{L}$ & 12 & 0.25 & 2.61 & 0.99 & 1.37 & Schmitt and Ness (2004) \\
\hline HR 1099 & $\mathrm{H}$ & 13 & 0.30 & 10 & 3.0 & 2.5 & Drake et al. (2001) \\
\hline HR 1099 & $\mathrm{R}$ & 14 & $\ldots$ & 15.6 & 3.9 & 3.7 & Brinkman et al. (2001) \\
\hline HR 1099 & $\mathrm{R}$ & 14.4 & 0.22 & 4.2 & 1.55 & 0.45 & Audard et al. (2001a) \\
\hline HR 1099 & $\mathrm{R}$ & 14.8 & 0.20 & 6.6 & 2.75 & 0.9 & Audard et al. (2003a) \\
\hline AR Lac & $\mathrm{H}$ & 15 & 0.74 & 2.16 & 0.81 & 0.95 & Huenemoerder et al. (2003) \\
\hline UX Ari & $\mathrm{R}$ & 15.1 & 0.14 & 13.4 & 4.0 & 2.21 & Audard et al. (2003a) \\
\hline II Peg & $\mathrm{H}$ & 16 & 0.15 & 14.9 & 7.4 & 2.7 & Huenemoerder et al. (2001) \\
\hline
\end{tabular}

\footnotetext{
${ }^{\mathrm{a} A}$ All abundance relative to solar photospheric values: Anders and Grevesse (1989) except for Fe: Grevesse and Sauval (1999)

${ }^{\mathrm{b}}$ Instrument: $\mathrm{R}=$ XMM-Newton RGS; H = Chandra HETGS; L = Chandra LETGS

${ }^{\mathrm{c}}$ Based on their method 2 using the SPEX database
}

transformed the abundances to refer to the solar values of Anders and Grevesse (1989) except for Fe, for which I adopted the value given by Grevesse and Sauval (1999). No attempt has been made to quote error estimates. Errors are extremely difficult to assess and include systematics from calibration problems and from the inversion method, and most importantly uncertainties in the atomic parameter tabulations. It is unlikely that any measurement represents its "true" value within better than $20 \%$. The average coro- 

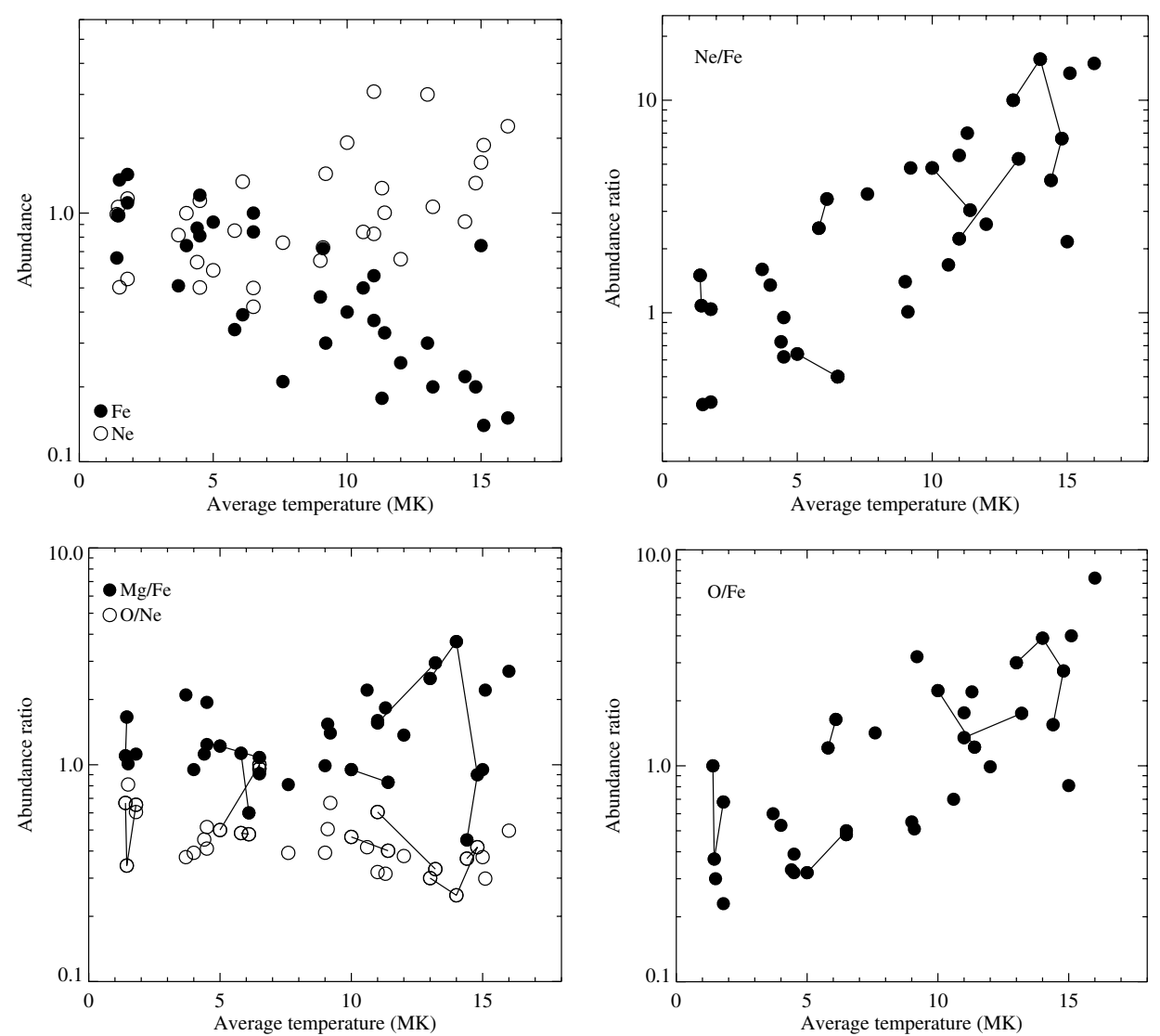

Fig. 37. Abundances as a function of the average coronal temperature from high-resolution spectroscopy with XMM-Newton and Chandra. Top left: Abundances of Fe (filled circles) and $\mathrm{Ne}$ (open circles), relative to solar photospheric values for the sample reported in Table 6. Top right: Similar, but the ratio between the $\mathrm{Fe}$ and $\mathrm{Ne}$ abundance is shown. Lower left: Similar, for the $\mathrm{Mg} / \mathrm{Fe}$ (filled) and $\mathrm{O} / \mathrm{Ne}$ ratios (open circles). Lower right: Similar, for the $\mathrm{O} / \mathrm{Fe}$ ratio. Lines connect points referring to the same star analyzed by different authors, or based on different observations

nal temperature was estimated either from the logarithmic $\operatorname{EMD}, \operatorname{EM}(\log T)$, or was calculated as the EM-weighted mean of $\log T$ in the case of numerically listed EMDs or results from multi- $T$ fits. Figure 37 shows the relevant trends. Clearly, the low-FIP elements $(\mathrm{Mg}, \mathrm{Fe})$ vary in concert, and so do the high-FIP elements $(\mathrm{O}, \mathrm{Ne})$. But the absolute $\mathrm{Fe}$ abundance significantly drops with increasing activity, and the $\mathrm{Ne} / \mathrm{Fe}$ ratio sharply increases as a consequence. The trend for $\mathrm{O} / \mathrm{Fe}$ is very similar, with ratios that are lower by typically a factor of two. The transition from the FIP to the IFIP pattern for $\mathrm{O} / \mathrm{Fe}$ occurs at average temperatures of about $7-10 \mathrm{MK} .^{10}$

\footnotetext{
10 The trends are independent of the spectral inversion method used to determine the abundances and the EMD; 17 spectra were fitted as a whole, while 17 spectra were analyzed with various iterative inversion methods using extracted line fluxes; both subsamples show identical trends.
} 


\subsection{Systematic uncertainties}

The abundance results presently available from high-resolution spectroscopy refer to data from different detectors, and various methods have been used to analyze the spectra. Some systematic deviations are found when we compare the reported abundance values (see Table 6). This comes as no surprise if we recall the discussion in Sect. 9.3 on the various difficulties inherent in the spectral inversion problem. Nevertheless, the situation is not as bad as it might appear, in particular once we review the actual needs for a physical model interpretation. I discuss examples from the recent literature, including some results from medium-resolution observations.

Capella has been a favorite target for case studies given its very high signal-to-noise spectra available from various satellites. Despite some discrepancies, the abundances derived from ASCA, EUVE (Brickhouse et al. 2000), BeppoSAX (Favata et al. 1997c), XMM-Newton (Audard et al. 2001b), and Chandra (Mewe et al. 2001) agree satisfactorily: Fe and $\mathrm{Mg}$ are close to solar photospheric values (see Sect. 16.5 below).

Several analyses have been presented for the RS CVn binary HR 1099 (for example by Brinkman et al. 2001; Audard et al. 2001a; Drake et al. 2001; Audard et al. 2003a). Although systematic differences are found between the derived abundances, different atomic databases (APEC, SPEX, HULLAC) and different instruments or instrument combinations (e.g., RGS+EPIC MOS on XMM-Newton, or HETGS on Chandra) were used during various stages of coronal activity (and at various stages of early instrument calibration efforts), making any detailed comparison suspect. For example, Audard et al. (2003a) showed conflicting results from two analyses that differ exclusively by their using different atomic databases. The overall trends, however, show gratifying agreement, also for several further magnetically active binaries. All results indicate low Fe $(0.2-0.3$ times solar photospheric for HR 1099), a high $\mathrm{Ne} / \mathrm{Fe}$ abundance ratio $(\approx 10)$, a high $\mathrm{Mg} / \mathrm{Fe}$ abundance ratio ( $\approx 2$ in several active stars), and an overall trend for increasing abundances with increasing FIP. The same conclusions were reported by Schmitt and Ness (2004) for an analysis of the Algol spectrum based on different reconstruction methods, the main uncertainty being due to large systematic errors in the atomic physics parameters.

Telleschi et al. (2004) systematically studied element abundances and EMDs for solar analogs at various activity levels, applying approaches that involve either spectral fits of sections of the spectra, or iterative reconstructions based on selected emission line fluxes. The two iteration methods yielded nearly identical abundances (Fig. 36). A more fundamental problem was recognized in an unbiased determination of line fluxes in the presence of line blends, and in the use of different spectroscopic databases.

Expectedly, there is less agreement between results from high-resolution data and analyses that make use of low- or medium-resolution observations (from, e.g., ASCA or BeppoSAX), but several trends are noteworthy. First, high $\mathrm{Ne} / \mathrm{Fe}$ abundance ratios are in fact found in a number of reports based on ASCA observations, even if the agreement is not always convincing. The following ratios have again been converted to the Anders and Grevesse (1989) and Grevesse and Sauval (1999) solar photospheric abundances (see also Table 6): For II Peg, Ne/Fe = 4.5 (ASCA, Mewe et al. 1997) vs. 15 (Chandra, Huenemoerder et al. 2001); for AR Lac, 3.4 (ASCA, White et al. 1994) vs. 2.2 (Chandra, Huenemoerder et al. 2003); for $\lambda$ And, 3.4 (ASCA, Ortolani et al. 1997) vs. 4.0-5.3 (XMM-Newton, Audard et al. 2003a) and 2.2 (Chandra, Sanz-Forcada et al. 2004); for 
HR 1099, 5.9 (ASCA, Osten et al. 2004) vs. 4.2-10 (Chandra, Audard et al. 2001a, 2003a; Drake et al. 2001); for Algol, 1.4-2.3 (ASCA, Antunes et al. 1994) vs. 2.6 (Chandra, $\mathrm{Schmitt}$ and Ness 2004). Similar trends apply to the $\mathrm{Mg} / \mathrm{Fe}$ ratios: For II Peg, $\mathrm{Mg} / \mathrm{Fe}=$ 1.2 (ASCA, Mewe et al. 1997) vs. 2.7 (Chandra, Huenemoerder et al. 2001); for AR Lac, 1.0-1.4 (ASCA, White et al. 1994; Kaastra et al. 1996) vs. 0.95 (Chandra, Huenemoerder et al. 2003); for $\lambda$ And, 1.2-2.2 (ASCA, Ortolani et al. 1997) vs. 2.5-3.0 (XMM-Newton, Audard et al. 2003a) and 1.6 (Chandra, Sanz-Forcada et al. 2004); for HR 1099, 1.6 (ASCA, Osten et al. 2004) vs. 0.45-2.5 (Chandra, Audard et al. 2001a, 2003a; Drake et al. 2001); for Algol, 0.8-1.2 (ASCA, Antunes et al. 1994) vs. 1.4 (Chandra, Schmitt and Ness 2004). The discrepancies are more severe and systematic for several other elements given the strong blending in low-resolution data (see, e.g., discussion in Huenemoerder et al. 2003).

In general, abundance ratios are more stable than absolute abundances that require a good measurement of the continuum level. This, in turn, requires an accurate reconstruction of the DEM. But what are we to make out of the residual uncertainties and discrepancies in abundance ratios from the interpretation of high-resolution data? It is perhaps important to recall the situation in the solar corona. There, abundances change spatially, and also in time as a given coronal structure ages (Feldman 1992; Jordan et al. 1998). As a further consequence, abundances are likely to vary with temperature across the corona. Second, the DEM represents a complex mixture of quiet plasma, evolving active regions, bright spots, and flares at many luminosity levels. A "best-fit" parameter derived from a spectrum therefore represents a sample mean related to a statistical distribution of the respective physical parameter. Whatever error ranges are reported, they inevitably refer to this sample mean whereas the actual spread across the physically distinct sources in a stellar corona remains presently unknown. A sound physical interpretation will have to address abundance ratios in specific coronal sources. In this context, excessive accuracy may well be meaningless for observational studies even if atomic physics problems were absent - the essence for further physical interpretation are trends such as those shown in Fig. 37.

\subsection{Coronal and photospheric abundances}

All coronal plasma ultimately derives from the respective stellar photosphere. Strictly speaking, therefore, we should define abundance ratios with respect to the underlying photospheric abundances. Unfortunately, these are all too often poorly known, or unknown altogether.

Photospheric metallicities, $Z_{\text {phot }}$, have been reported for a number of RS CVn binaries (see, among others, Randich et al. 1993, 1994; the latter authors, however, cautioned against the strict use of their measurements as true metallicity indicators). Unexpectedly, at least for population I stars, many of these systems turn out to be rather metal poor. If the coronal abundances are compared with these photospheric metallicities, the putative coronal underabundances may disappear entirely. This seems to be the case for Capella (Favata et al. 1997c; Brickhouse et al. 2000). Similarly, White et al. (1994) reported comparable coronal and photospheric abundances for the RS CVn binary AR Lac, and Favata et al. (1997b) found the coronal metallicity of VY Ari (0.4 times solar) to be in the midst of measured photospheric metallicities of RS CVn binaries as 
a class. Ortolani et al. (1997) measured, from low-resolution ROSAT data, a coronal metallicity, $Z_{\text {cor }}$, in $\lambda$ And that is nearly consistent with the stellar photospheric level, although ASCA data indicated lower metallicity, and several discrepant sets of photospheric abundances have been published (see Audard et al. 2003a). The trend agrees with abundance ratios being the same in the corona and in the photosphere of this star (Audard et al. 2003a). The RS CVn binary CF Tuc has revealed particularly low coronal metal abundances of 0.1 times solar photospheric values (Schmitt et al. 1996d; Kürster and Schmitt 1996), but again this finding is accompanied by low measured photospheric abundances (Randich et al. 1993). The same appears to apply to the RS CVn/BY Dra binaries TY Pyx $\left(Z_{\text {cor }} \approx 0.5-0.7, Z_{\text {phot }} \approx 0.63\right.$, Franciosini et al. 2003; Randich et al. 1993) and HD $9770\left(Z_{\text {cor }} \approx 0.5-0.6, Z_{\text {phot }} \approx 0.30-0.35\right.$, Tagliaferri et al. 1999). Although S. Drake et al. (1994a) found that the spectrum of the giant $\beta$ Cet cannot be fitted even when assuming the known photospheric abundances, Maggio et al. (1998) reported rough agreement between photospheric ( $90 \%$ confidence ranges for $\mathrm{Si}$ : 1.4-3.2, Fe: 0.7-2.5 times solar) and coronal abundances (Si: 0.9 [90\%: 0.3-2.4], Fe: 0.7 [90\%: 0.5-2] times solar), albeit with large uncertainties.

But new complications have surfaced. Ottmann et al. (1998) critically reviewed previous photospheric abundance determinations and revisited the problem using a sophisticated spectroscopic approach to derive all relevant stellar parameters self-consistently. They challenged reports of very low photospheric metallicities in otherwise normal stars, finding at best mild underabundances (e.g., 40-60\% solar Mg, $\mathrm{Si}$, and Fe values for II Peg and $\lambda$ And), and metal abundances very close to solar for young, nearby solar analogs ( $\kappa^{1}$ Cet and $\pi^{1} \mathrm{UMa}$ ). An application of the technique to a solar spectrum returned the correct solar values. These more solar-like values seem closer to what should be expected for nearby, young or intermediately old population I stars (Feltzing et al. 2001). The apparent photospheric underabundances in active stars may be feigned by chromospheric filling-in of the relevant lines and due to photometric bias from large dark-spot areas, an explanation that Randich et al. also put forward to explain their significantly differing abundances for the two components in some active binaries. In the light of these reports, several authors returned to recover significantly depleted coronal abundances also relative to the respective photospheres.

An illustrative example is HR 1099. Recent coronal abundance determinations for this star converge to subsolar values in particular for Fe (between 0.2-0.3, Audard et al. 2001a, 2003a; Drake et al. 2001), and these values seem to superficially agree with an Fe abundance of $\approx 0.25$ measured for the photosphere (Randich et al. 1994). On the other hand, Strassmeier and Bartus (2000) and Savanov and Tuominen (1991) reported photospheric Fe abundances of, respectively, 0.6-0.8 and 1.0 times solar, which would be in agreement with values expected from the age of this system (Drake 2003a), thus arguing in favor of a real depletion of the coronal Fe. Similarly, Covino et al. (2000) found $Z_{\text {cor }} \approx 0.2$ for II Peg (Mewe et al. 1997 even give $\mathrm{Fe} / \mathrm{H} \approx 0.1$ ), whereas the photosphere is 3 times richer in metals $\left(Z_{\text {phot }}=0.6\right.$ according to Ottmann et al. 1998). And Huenemoerder et al. (2001) reported a coronal Fe abundance four times below photospheric for II Peg, and various abundances are found at $60 \%$ of the photospheric values in AR Lac (Huenemoerder et al. 2003).

Particularly interesting test samples are nearby, young solar analogs for which reliable photospheric abundances have been reported, not too surprisingly being consistent 
with solar photospheric values (e.g., $\mathrm{Mg}, \mathrm{Si}$, and Fe given by Ottmann et al. 1998 for $\kappa^{1}$ Cet and $\pi^{1}$ UMa; several elements [Al, Ca, Fe, Ni] reported by Vilhu et al. 1987 for AB Dor; further values listed by Cayrel de Strobel et al. 2001 and references therein also for $\chi^{1}$ Ori; with several individual elements being close to solar, large discrepancies appear rather unlikely for other elements). A corresponding coronal X-ray study based on XMM-Newton spectroscopy, however, indicated significant overall metal depletion on the one hand, where the Fe depletion is stronger for higher activity levels, and a relative FIP bias on the other hand; the latter changes from "normal" to "inverse" with increasing activity level. Both trends disagree with photospheric patterns (Güdel et al. 2002c; Telleschi et al. 2004).

Other important test cases are stars that reveal strong, undisputed photospheric deviations from the solar abundance pattern. "Super-metal rich" stars are stars with measured photospheric Fe abundances $[\mathrm{Fe} / \mathrm{H}] \geq 0.2$ (logarithmic, relative to the solar photosphere). Maggio et al. (1999) observed two extreme cases with $[\mathrm{Fe} / \mathrm{H}]=0.25$ (30 Ari B) and $[\mathrm{Fe} / \mathrm{H}]=0.305$ ( $\eta$ Boo). Surprisingly, not only were the relative coronal abundances $[\mathrm{Mg} / \mathrm{Fe}],[\mathrm{Si} / \mathrm{Fe}]$, and $[\mathrm{O} / \mathrm{Fe}]$ found to be close to the abundances of otherwise similar field stars with near-solar composition, but the absolute coronal $[\mathrm{Fe} / \mathrm{H}]$ abundance was also derived to be near-solar. The authors suspected a number of artefacts related to low-resolution CCD spectroscopy, however. At the opposite end, namely in metal-poor Population II stars, the simple lack of metals available in the stellar material should reflect strongly in the coronal emission. A striking case was presented by Fleming and Tagliaferri (1996): The binary HD 89499 with $[\mathrm{Fe} / \mathrm{H}]=-2.1$ shows X-ray spectra that are essentially line-free, that is, they are dominated by bremsstrahlung. Because the emissivity of such plasma is much lower than the emissivity of a plasma with solar composition, the material can efficiently be heated to higher temperatures, and indeed all plasma is detected at $T \approx 25 \mathrm{MK}$, with no significant amounts of cooler material.

There are nevertheless important examples of stars that show no indication of coronal metal depletion also in the light of new photospheric abundance measurements. Drake and Kashyap (2001) found a slight enhancement of coronal vs. photospheric abundances in the intermediately active $\xi$ Boo A, in agreement with a measured solar-like FIP effect. A trend for smaller metal deficiencies toward less active stars has been noted earlier (Singh et al. 1995), and the least active stars such as Procyon (Drake et al. 1995b; Raassen et al. 2002) and $\alpha$ Cen generally show solar-like metallicities, with the additional possibility of a solar-type FIP effect (Drake et al. 1997; Mewe et al. 1998a,b; Raassen et al. 2003a). The very active late-F star HD 35850 with measured solar photospheric Fe abundance also requires near-solar or only slightly subsolar abundances (Gagné et al. 1999) although there is disagreement with the analysis given by Tagliaferri et al. (1997). A few further examples with near-photospheric composition were discussed by Sanz-Forcada et al. (2004).

The above discussion amply illustrates the inconclusive and unsatisfactory present observational status of the field. The easy access to coronal abundance diagnostics makes further, comprehensive photospheric abundance determinations very desirable. 


\subsection{Flare metal abundances}

Abundances may change in particular in flares because the evaporation process brings new photospheric or chromospheric material into the corona. Peculiar effects have also been noted in some solar flares, as described in Sect. 16.1.

There was some early evidence for enhanced or depleted Fe abundances in large stellar flares, mostly on the basis of the line-to-continuum ratio for the $\mathrm{Fe} \mathrm{K} \alpha$ line at $6.7 \mathrm{keV}$. Stern et al. (1992a) found an equivalent width of only $25 \%$ of the expected value in a flare on Algol, possibly indicating a corresponding Fe underabundance. A similar effect was noted by Tsuru et al. (1989). In both cases, suppression due to resonance scattering was discussed as an alternative explanation. Doyle et al. (1992a) reported a very low Fe abundance (33\% solar) during a large flare on II Peg. Ottmann and Schmitt (1996) noticed an increase of the coronal metallicity from 0.2 to $0.8 Z_{\odot}$ during a giant flare on Algol observed by ROSAT in the $0.1-2.4 \mathrm{keV}$ range, although the low spectral resolution makes such measurements problematic, for example if the dominant plasma found in simple spectral fits is far from the relevant maximum line formation temperatures. White et al. (1986), on the other hand, concluded for a flare on Algol that the Fe abundance was within $20 \%$ of the solar photospheric value. Further reports of unusual equivalent widths of the Fe $\mathrm{K} \alpha$ line have been reported by Doyle et al. (1991), Tsuru et al. (1992), and Vilhu et al. (1993).

Higher-resolution broad-band spectra permitted measurements of other individual elements and more complicated thermal structures. Güdel et al. (1999), Osten et al. (2000) and Audard et al. (2001a) investigated the temporal evolution of several abundances during large, gradual flares on RS CVn binaries. They all found increases of low-FIP element abundances (e.g., of $\mathrm{Fe}, \mathrm{Si}, \mathrm{Mg}$ ) during the flare maximum, and a rapid decay back to pre-flare values during the later phases of the flare. In contrast, a large flare on Proxima Centauri revealed no FIP-related abundance evolution although all abundances appeared to be elevated in proportion during a narrow interval around the flare peak (Güdel et al. 2004). Similarly, Osten et al. (2003) found an increase of the $\mathrm{Fe}$ abundance during a flare on $\sigma^{2} \mathrm{CrB}$ by a factor of two, while the other elements increased accordingly, with no systematic trend related to the FIP.

Another, albeit less detailed, approach involves the monitoring of the "global" metallicity $Z_{\text {cor }}$, i.e., abundances are assumed a priori to vary in proportion. The metallicity is then predominantly driven by $\mathrm{Fe}$, in particular if the $\mathrm{Fe} \mathrm{K} \alpha$ complex at $6.7 \mathrm{keV}$ is accessible. Mewe et al. (1997), Tsuboi et al. (1998), Favata and Schmitt (1999), Favata et al. (2000a), Güdel et al. (2001b), Covino et al. (2001), and Osten et al. (2002) noted peak enhancements of $Z_{\text {cor }}$ by factors of about 3-4 during flares on II Peg, V773 Tau, Algol, EV Lac, AB Dor, G1 355, and EI Eri, respectively. In contrast, Maggio et al. (2000) and Franciosini et al. (2001) reported the absence of a significant metallicity enhancement in flares on AB Dor and UX Ari, respectively, although the upper limits were still consistent with enrichment factors of $\approx 3$.

Despite some variations of the theme, it seems to be certain that flares can change the elemental composition of the plasma, and the trend is toward increasing metallicity in flares on active stars that otherwise show metal-depleted coronae. Some ideas for models will be discussed in the next section. 


\subsection{Theoretical models for abundance anomalies}

It has proven particularly difficult to interpret the abundance anomalies in stellar coronae. This should come as no surprise given that the situation is not all that different for the solar corona, despite the abundance of sophisticated but competing models (Hénoux 1995). I briefly list a few suggestions that, however, all require further elaboration; another recent summary has been given by Drake (2003b).

Stratification of the atmosphere. Mewe et al. (1997) proposed that the abundance features observed in active stars are due to the different scale heights of different ions in a hydrostatic coronal loop, depending on mass and charge. As a result, the ion distribution in a magnetic loop is inhomogeneous and the line-to-continuum ratio depends on the scale height of a specific ion. After flares, the settling to equilibrium distributions is expected to occur on time scales of hours. This model cannot, however, explain the high $\mathrm{Ne}$ abundances in active stars because of the mass dependence of the proposed stratification: $\mathrm{Ne}$ has a mass intermediate between $\mathrm{O}$ and $\mathrm{Mg}$ !

Coronal equilibrium. All determinations of element abundances strongly rely on the assumption of collisional equilibrium. The thermalization time is usually short enough to justify this assumption. If the observed coronal emission is, however, driven by a sequence of small flares (Sect. 13.6) then a number of further conditions would have to apply, which are difficult to assess. Whether such effects could change the measured abundances is currently unknown (see also the discussion in Huenemoerder et al. 2003).

"Anomalous flares". High Ne abundances are occasionally seen on the Sun during "Ne rich" flares (Schmelz 1993). Shemi (1991) argued that the high photoionization cross section of Ne makes it prone to efficient ionization by X-ray irradiation of the chromosphere during flares, thus making Ne behave like a low-FIP element in the solar corona. While this model explains the Ne enrichment, it does not address the apparent underabundance of the low-FIP element Fe.

Evaporation. The peak metallicities observed in large flares seem to be bounded by $Z \lesssim Z_{\odot}$. Assuming that most population I stars in the solar neighborhood have photospheric abundances similar to the Sun, then the coronal abundance increase suggests that new chromospheric/photospheric material is supplied to the corona, most likely by the chromospheric evaporation process (Ottmann and Schmitt 1996; Mewe et al. 1997; Güdel et al. 1999). The selective low-FIP element enhancement during some large flares (Güdel et al. 1999; Osten et al. 2000; Audard et al. 2001a) is further compatible with a chromospheric evaporation model presented by Wang (1996) in which the evaporation induces upward drifts of electrons and protons. These particles then efficiently drag chromospheric ions (i.e., preferentially low-FIP elements) in an ambipolar diffusion process, while they leave neutral (preferentially high-FIP) elements behind.

Stellar evolution. Schmitt and Ness (2002) found N/C abundance ratios that are enhanced by factors of up to $40 \mathrm{in} \mathrm{Algol} \mathrm{and} \mathrm{in} \mathrm{a} \mathrm{sample} \mathrm{of} \mathrm{(sub-)giants.} \mathrm{They} \mathrm{attributed}$ this anomaly to an enrichment of $\mathrm{N}$ from mass transfer in Algol, and from dredge-up of $\mathrm{N}$ in the evolving giants. This has been quantitatively worked out in the context of mass loss evolution and convective dredge-up by Drake (2003a) for Algol. The N enrichment and $\mathrm{C}$ depletion then essentially come from nuclear processing through the $\mathrm{CN}$ cycle in the secondary star, followed by strong mass loss from the outer convection zone and dredge-up of $\mathrm{N}$ enriched material. 
He enrichment. An increased abundance of He relative to $\mathrm{H}$ due to some fractionation process in the chromosphere would increase the continuum level and therefore decrease the line-to-continuum ratios, thus leading to an apparent underabundance of the metals (Drake 1998). Helium enhancements by factors of a few are required, however (Rodonò et al. 1999), up to levels where He would be the most abundant element of the plasma (Covino et al. 2000). FIP-related trends are not explained by this model.

Non-thermal emission. I mention here another possibility that may lower all abundances in general, and in particular the Fe abundance if measured from the Fe K complex at $6.7 \mathrm{keV}$. If the continuum level is enhanced by an additional power-law component due to impact of non-thermal electrons in the chromosphere, then the equivalent width of the line is below predictions, simulating a "depleted Fe abundance" (Vilhu et al. 1993). Observationally, quite high signal-to-noise ratios and good spectral resolution may be required to rule out non-thermal contributions. Such a "non-thermal" model is interesting because appreciable suppression of the overall metallicity is found particularly in stars that are strong non-thermal radio emitters (Güdel et al. 2002c).

There is another coincidence between the appearance of non-thermal electrons and element abundance anomalies. The change from an inverse-FIP bias to a normal solartype FIP effect occurs at quite high activity levels, close to the empirical saturation limit for main-sequence stars (Güdel et al. 2002c). At the same time, non-thermal radio emission drops sharply (much more rapidly than the X-rays). If electrons continuously propagate into the chromosphere at a modest rate without inducing strong evaporation, then a downward-pointing electric field should build up. This field should tend to trap chromospheric ions, i.e., predominantly the low-FIP elements, at low levels while neutrals, i.e., predominantly high-FIP elements, are free to drift into the corona. As radio emission disappears in lower-activity stars, the low-FIP element suppression disappears, and a solar-type FIP effect may build up, for whatever physical reasons, in analogy to the solar case.

\section{X-ray emission in the context of stellar evolution}

X-ray emission offers easy access to stellar evolution studies because magnetic activity is governed by various stellar parameters such as convection zone depth and rotation that change gradually as a star evolves. Evolutionary studies have been based either on nearby, modestly-sized but well-defined samples of stars or on large statistical samples from open clusters. I will first describe some principal results from the first approach and then concentrate on open cluster studies.

\subsection{Main-sequence stars}

The X-ray luminosity of field F-G stars clearly decays monotonically with age. Studies of young open clusters (Sect. 17.5) indicate a slow decay during the initial few $100 \mathrm{Myr}$ of a solar-like star, with $L_{X}$ scaling approximately like the inverse of the age (Patten and Simon 1996). In contrast, somewhat older field stars show a steep decay toward higher ages. For nearby F-G main-sequence stars for which approximate ages have been derived mostly from their surface Li abundance, moving group membership, or rotation (once 
sufficiently converged toward a mass-dependent value), the X-ray luminosity decays like

$$
L_{X} \approx(3 \pm 1) \times 10^{28} t^{-1.5 \pm 0.3} \quad\left[\mathrm{erg} \mathrm{s}^{-1}\right]
$$

where the age $t$ is given in Gyr (Maggio et al. 1987; Güdel et al. 1997a). The same trend is found in open clusters (see Sect. 17.5 and Fig. 41 below). It is quite certain that this decay law reflects the slowing of the rotation rate with age rather than some intrinsic dynamo ageing (Hempelmann et al. 1995). It must therefore derive directly from the combination of the rotation-age relationship (e.g., Soderblom et al. 1993) and the rotation-activity dependence (Sect. 5).

As the star ages and its overall luminosity decreases, the EM distribution shifts to cooler temperatures, with a rapid decrease in particular of the hot plasma component - see Fig. 38 (and Sect. 9.4 and 9.5). A possible cause of the temperature decrease are the less efficient coronal magnetic interactions in less active stars given their lower magnetic filling factors, and consequently a less efficient heating, or a lower rate of flares that produce high-temperature plasma (Güdel et al. 1997a; Güdel 1997).

It is reassuring that the Sun and its near-twin, $\alpha$ Cen A, behave very much alike, both in terms of coronal temperature and $L_{X}$ (Mewe et al. 1995, 1998a; Drake et al. 1997). The similarity between $\alpha$ Cen A and the Sun in spectral type, rotation period and age provides a convenient approximation to the "Sun as a star" (Golub et al. 1982; Ayres

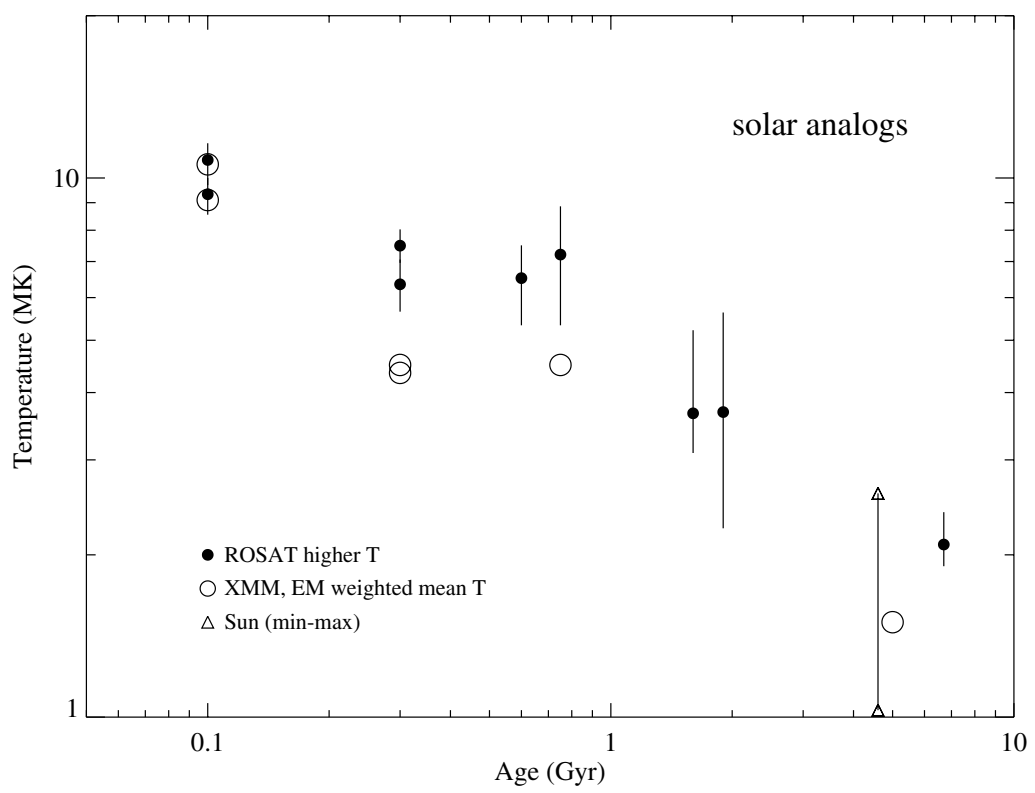

Fig. 38. Decay of coronal temperature with age for solar-type stars. The temperatures of the hotter component in the G star sample of Güdel et al. (1997a) and Güdel et al. (1998) are shown (filled circles). Solar data are from Peres et al. (2000) and illustrate the range from activity minimum to maximum (triangles). The open circles represent the EM-weighted logarithmic average of $T$ as calculated from EMDs (Telleschi et al. 2004) or from multi- $T$ spectral fits (for $\alpha$ Cen at $t=5 \mathrm{Gyr}$, Raassen et al. 2003a) 
et al. 1982). Seen from this perspective, the full-disk DEM has cooled to a distribution between 1-5 MK with a peak at 3 MK (Mewe et al. 1998a; Raassen et al. 2003a), representative of a star in which a mixture of active regions, quiet regions, and coronal holes prevail (Orlando et al. 2000; Peres et al. 2000, see Sect. 11.4).

When we move to lower-mass stars, the picture changes gradually. A progressively larger fraction of stars is found at relatively high activity levels toward lower masses, in particular among M dwarfs. This is a consequence of the smaller spin-down rate for less massive stars (Fleming et al. 1988, 1995; Giampapa et al. 1996): low-mass stars stay active for a longer time. This may be related to a different dynamo being in operation in late $\mathrm{M}$ dwarfs where the radiative core is small or absent altogether (Giampapa et al. 1996). The more rapid spin-down of more massive dwarfs is, in turn, illustrated by the paucity of extremely active $\mathrm{G}$ and early $\mathrm{K}$ stars in the immediate solar vicinity; the nearest examples are found at distances of 15-30 pc with ages of no more than $\approx 100 \mathrm{Myr}$ (e.g., the K0 V rapid rotator AB Dor [Vilhu et al. 1993] or the young solar analogs EK Dra [Güdel et al. 1995] and 47 Cas B [Güdel et al. 1998]). For M dwarfs, we find a clear ageing trend only when we look at much larger age ranges: their X-ray luminosity significantly decays in concert with their metallicity from young to old disk population stars (Fleming et al. 1995; metallicity generally decreases with increasing population age). This trend continues toward the oldest, halo population $\mathrm{M}$ dwarfs that reveal significantly softer X-ray spectra than young and old disk stars (Micela et al. 1997a; see also Barbera et al. 1993).

\subsection{Giants}

The giant and supergiant area of the HRD is more complicated because evolutionary tracks are running close to each other and partly overlap. An overview of the relevant area is shown in Fig. 39. As cool stars evolve from the main sequence to the giant branch, the deepening of the convection zone may temporarily increase the coronal magnetic activity level (Maggio et al. 1990). In general, however, the X-ray activity of stars with masses $\lesssim 1.5 M_{\odot}$ further decreases as they move redward in the HRD (Pizzolato et al. 2000) because these stars begin their evolution toward the giant branch as slow rotators on the main sequence, and the increasing radius further slows their rotation rate.

The evolution of magnetic activity is different for more massive stars because they start their evolution off the MS as rapidly rotating $\mathrm{O}, \mathrm{B}$, and A stars. As convection suddenly sets in, a dynamo begins to operate in the stellar interior, and a magnetized wind starts braking the star in the region of early G-type giants and supergiants (Hünsch and Schröder 1996; Schröder et al. 1998). These "first crossing stars" develop maximum X-ray activity among $\mathrm{G}$ giants $\left(L_{X} \lesssim 10^{31} \mathrm{erg} \mathrm{s}^{-1}\right.$, Maggio et al. 1990; Micela et al. 1992; Scelsi et al. 2004). The break in rotation period is located significantly blueward of the location where X-rays weaken (Gondoin et al. 1987). This is because the convection zone deepens and, possibly, differential rotation strengthens as the star evolves redward; both trends help strengthen the dynamo against the slowing rotation (Gondoin 1999; Pizzolato et al. 2000). As a consequence, one finds no unique rotation-activity relation for the complete ensemble of giants, and some of the more rapidly rotating late giants keep extreme activity levels in X-rays (Gondoin et al. 2002; Gondoin 2003a,b,c, 2004b). 


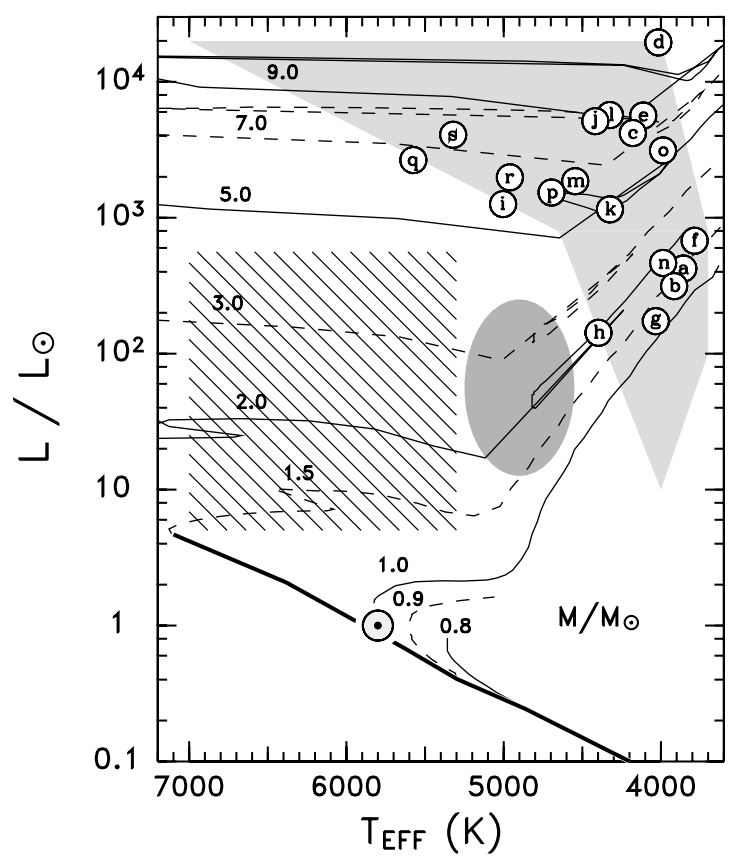

Fig. 39. Main features of the giant HRD. The hatched region schematically illustrates the Hertzsprung gap area, while the gray oval region marks the area of the "clump stars". The polygonal region in the upper right corner shows the approximate range of the cool-wind stars. The original dividing line is the lower left hand edge of this area. Individual circles mark the loci of "hybrid" stars. Evolutionary tracks are given for a variety of masses (figure courtesy of T. Ayres, after Ayres 2004)

Only at $B-V \gtrsim 0.9-1$ do we find a gradual decrease of $L_{X}$, up to $B-V \approx 1.1$ $(\approx \mathrm{K} 1 \mathrm{III})$. Toward later spectral types, $\mathrm{X}$-ray emission drops to very low values.

The X-ray emission of early F giants and subgiants is deficient with respect to transition region line fluxes (Simon and Drake 1989; Ayres et al. 1995, 1998; Fig. 5). Simon and Drake (1989) therefore argued in favor of acoustic coronal heating for the warmer stars that show no appreciable magnetic braking by a wind, and solar-like magnetic heating for the cooler stars in which magnetic braking is effective. In contrast, Ayres et al. (1998) proposed very extended $\left(L \approx R_{*}\right.$ ) coronal loops with long ( $\approx 1$ day) filling durations and cooling times that favor a redistribution of the energy into the transition region where it is efficiently radiated. Such loops may be remnants of global magnetospheres as proposed in different contexts for hotter MS stars, coexisting with a growing solar-type corona that is generated by a dynamo in the deepening convection zone. The systematic behavior is less clear in supergiants given their smaller number statistics; the outstanding early-type example among single Hertzsprung gap supergiants is $\alpha$ Car that shows an appreciable $L_{X}$ despite its shallow convection zone (F0 II, $\log L_{X}=29.8$, Maggio et al. 1990).

As giants evolve, and at least the less massive examples undergo a He-flash, they gather in the "clump" region of the HRD, at roughly $0.95 \leq \mathrm{B}-\mathrm{V} \leq 1.2$ (K giants, 
see Fig. 39). Again, many of these sources are detected in X-rays, albeit at relatively weak levels, with $L_{X}$ of a few times $10^{27} \mathrm{erg} \mathrm{s}^{-1}$ (Schröder et al. 1998). Strong flaring activity before and after the He-flash testifies to the ability of the dynamo to survive the internal reconfiguration (Schröder et al. 1998; Ayres et al. 1999, 2001a).

In this context, the Capella binary is particularly interesting since it contains a primary clump giant (G8 III) and a presumably co-eval Hertzsprung gap star (G1 III) of about equal mass $\left(\approx 2.6 M_{\odot}\right)$. The FexXI $\lambda 1354$ line shows considerable variability on timescales of several years in the clump giant, but not in the Hertzsprung gap star (Johnson et al. 2002; Linsky et al. 1998). The former authors suggested that long-term variability, for example induced by dynamo cycles, occurs only in the clump phase where a deep convection zone has built up.

Another example is the quadruplet of the nearly co-eval Hyades giants $(\gamma, \delta, \epsilon$, and $\left.\theta^{1} \mathrm{Tau}\right)$. The four stars are found at very similar locations in the HRD, but show a spread in $L_{X}$ over orders of magnitude (Micela et al. 1988; Stern et al. 1992c). A possible explanation is, apart from contributions by companions, that the high-activity stars are first crossers while the others have already evolved to the clump area (Collura et al. 1993).

\subsection{Dividing lines}

From ultraviolet observations of chromospheric and transition region lines in giants, Linsky and Haisch (1979) proposed a dividing line in the giant and supergiant area of the HRD roughly at V-R $=0.7-0.8$, separating stars with chromospheres and hot transition regions to the left from stars that exhibit exclusively chromospheric lines, to the right. A steep gradient in transition region fluxes is found when one moves from spectral type $\mathrm{G}$ towards the dividing line, located roughly between K2 III and G Ib (Haisch et al. 1990c). The absence of warm material in the later-type stars suggested an absence of coronal material as well, which was soon confirmed based on Einstein observations (Ayres et al. 1981a; Haisch and Simon 1982; Maggio et al. 1990). ROSAT observations deepened this conclusion, converging to a more "perpendicular" dividing line at spectral type $\approx$ K3 between luminosity classes II-IV (Haisch et al. 1991b).

The dividing line roughly corresponds to the "onset" of massive cool winds toward M giants and supergiants (Reimers et al. 1996). This coincidence may be a consequence of the cooler stars carrying predominantly open magnetic field lines that produce stellar winds somewhat similar to solar coronal holes (Linsky and Haisch 1979). Plasma that resides in open magnetic regions is necessarily cool in such stars because the escape temperature is much smaller than on the Sun, and this leads to small Alfvén-speed scale heights and thus to strong winds via Alfvén-wave reflection (Rosner et al. 1991). Why cooler stars should show predominantly open field lines is unexplained. A shift from an $\alpha \omega$ dynamo to a turbulent dynamo in cooler stars (Gondoin et al. 1987; Gondoin 1999) could possibly change the magnetic field structure.

An $\alpha^{2}$ dynamo that relies on convection close to the surface but not on rotation may indeed be a favorable option given the slow rotation of red giants (Ayres et al. 2003a). In this case, one expects that smaller-scale loops are generated, and this has two consequences (e.g., Rosner et al. 1995; Schrijver and Haisch 1996): First, the larger thermal pressure in low-lying hot loops will open them up if the magnetic field strength 
is not sufficiently high, thus draining the available energy into a stellar wind. Second, Antiochos et al. (1986) proposed that the low gravity of the cooler and therefore, larger stars to the right of the dividing line allows for new static cool loop solutions with $T<10^{5} \mathrm{~K}$ and small heights (after Martens and Kuin 1982; Antiochos and Noci 1986), while hot X-ray loops with heights less than the (large!) pressure scale height at $10^{5} \mathrm{~K}$ grow unstable and are therefore not radiating. In this model, then, the hot magnetic corona is replaced by a cooler but extended transition region. Any hot material detected from stars to the right of the dividing line or from "hybrid stars" (see below) may entirely be due to short-term flaring (Kashyap et al. 1994). The above two instabilities apply to warmer stars as well: Because the magnetic loop size is restricted by the convection zone depth which is small for F stars, there is a regime in that spectral range where the above arguments apply similarly, i.e., hot coronae will become unstable (Schrijver and Haisch 1996).

Even if a magnetically confined X-ray corona is present in giants, its X-ray emission may be efficiently absorbed by the stellar wind so that the dearth of coronal emission in M giants may only be apparent (Maggio et al. 1990). Since several non-coronal giants have been found to maintain transition regions up to $10^{5} \mathrm{~K}$, Ayres et al. (1997) proposed that coronal loops are in fact submerged in the cool chromospheric and $\mathrm{CO}$ layers and are thus absorbed. The reason for an extended, not magnetically trapped chromosphere is rooted in the 40-fold larger pressure scale height on red giants such as Arcturus compared to the Sun. The model of "buried" coronae would again be aided if the dynamo in these stars favored short, low-lying loops (Rosner et al. 1995). This view has gained strong support from the recent detection of very weak X-ray emission from the K1 III giant Arcturus, with $L_{X} \approx 1.5 \times 10^{25} \mathrm{erg} \mathrm{s}^{-1}$ (Ayres et al. 2003a); Arcturus was previously thought to be X-ray dark (Ayres et al. 1981a, 1991). Ayres et al. (2003a) also reported the presence of transition region lines that are absorbed by overlying cooler material that acts like a "cool absorber".

Hünsch and Schröder (1996) compared the dividing line with evolutionary models and concluded that it is actually nearly parallel to the evolutionary tracks in the giant domain. They suggested that the drop of X-ray activity toward cooler stars is gradual and is a direct function of mass. In this picture, the coolest $\mathrm{M}$ giants with masses $\lesssim 2 M_{\odot}$ and ages around $10^{9} \mathrm{yr}$ are X-ray faint simply because they had spun down on the MS before and are thus no longer able to drive a dynamo (Ayres et al. 1981b; Haisch and Simon 1982; Hünsch and Schröder 1996; Schröder et al. 1998) while the warmer giants, descendants of $M>2 M_{\odot}$ main-sequence stars, have retained more angular momentum.

\subsection{Hybrid stars}

The situation is somewhat less clear for evolved supergiants and bright giants (luminosity class II) given the small statistics at hand, but there is now evidence against a clear dividing line in this region of the HRD (Reimers et al. 1996). While the wind dividing line sharply bends to the left in the HRD as most bright giants and supergiants show strong winds (Fig. 39), the so-called "hybrid stars" are formally right of a vertical dividing line extended from the giant region, but they show indications both of cool winds and $\approx 10^{5} \mathrm{~K}$ transition region material (Hartmann et al. 1980). After first X-ray detections (Brown et al. 1991; Haisch et al. 1992), it soon became evident that X-ray emitting hybrids 
are common across the regime of bright giants and supergiants of luminosity class I-II (Reimers and Schmitt 1992; Reimers et al. 1996; Hünsch et al. 1996). The co-existence of hot coronae and cool winds in hybrids renders them pivotal for understanding the physics of stellar atmospheres in this region of the HRD.

The X-ray luminosities of hybrids are relatively modest, with $L_{X}=10^{27}-10^{30} \mathrm{erg} \mathrm{s}^{-1}$ (Reimers et al. 1996; Ayres 2004); like other luminosity-class II stars, they are X-ray deficient compared to giants or main-sequence stars (Ayres et al. 1995; Reimers et al. 1996; Ayres 2004; Fig. 5), but at the same time they show very high coronal temperatures. The X-ray deficiency syndrome of supergiants is probably the same as that of F-type giants and subgiants (Simon and Drake 1989, Sect. 17.2). Indeed, the transformation from X-ray deficient to "solar-like" stars occurs at progressively later spectral types as one moves to higher luminosity classes, eventually encompassing almost the complete cool half of the supergiant HRD (Ayres et al. 1995). The co-existence of winds and coronae may be the result of the very rapid evolution of hybrid stars: The X-ray emission may compete with cool-wind production as the dynamo activity persists. In the picture of Hünsch and Schröder (1996), then, hybrids are X-ray sources because their masses are $>2 M_{\odot}$, i.e., they start out as fast rotators on the main-sequence and keep their dynamo while developing strong winds. The X-ray dividing line defined by spun-down low-mass stars does simply not reach up to these luminosity classes.

\subsection{Evolution of X-ray emission in open stellar clusters}

\subsubsection{Overview}

Open clusters have become instrumental for the study of stellar coronae and their longterm evolution for several reasons: i) They provide large samples of nearly co-eval stars spread over a broad mass range that encompasses all types of cool MS stars and possibly brown dwarfs, in the statistical proportion dictated by the processes of star formation; ii) their ages are rather well known from their distribution on the HRD; ii) their surface chemical composition is very likely to be constant across the stellar sample. Open clusters are therefore ideal objects with which to test theories of stellar evolution and, in particular, systematic dependences between rotation, activity, and age. While some of the issues have already been covered in the section on rotation (Sect. 5), the present section emphasizes specific evolutionary effects and sample studies made with open clusters.

Table 7 summarizes open cluster studies in the literature, also listing - if available - median X-ray luminosities for the spectral classes F, G, K, and M. The colors were not defined identically by all authors, but the large spread of $L_{X}$ and the statistical uncertainties will make this a rather negligible problem. Also included are clusters in star-forming regions. Because these stars are not yet on the main sequence, comparing them with MS clusters based on color may be misleading. For the sake of definition, I have lumped together all T Tau stars from such samples in the " $\mathrm{K}$ star" column unless more explicit information was available. Several of the given ages (mostly from the Lausanne open cluster database) must be treated as tentative.

Figure 40 shows the distribution of $L_{X}$ and $L_{X} / L_{\text {bol }}$ as a function of $B-V$ for the Pleiades (age $\approx 100 \mathrm{Myr}$ ) and the Hyades (age $\approx 700-800 \mathrm{Myr}$ ). The older 
Table 7. Open cluster studies: Luminosities and bibliography

\begin{tabular}{|c|c|c|c|c|c|c|}
\hline \multirow[t]{2}{*}{ Cluster } & \multirow[t]{2}{*}{ Age (Myr) } & \multicolumn{4}{|c|}{ Median $L_{X}$ for spectral class } & \multirow[t]{2}{*}{ References $^{\mathrm{a}}$} \\
\hline & & $\mathrm{F}$ & $\mathrm{G}$ & $\mathrm{K}$ & M & \\
\hline NGC 1333 & $<1$ & $\ldots$ & $\ldots$ & $\ldots$ & $\ldots$ & 1 \\
\hline$\rho$ Oph & $0.1-1$ & $\ldots$ & $\ldots$ & 30.4 & $\ldots$ & 2 \\
\hline Serpens & 1 & $\ldots$ & $\ldots$ & $\ldots$ & $\ldots$ & 3 \\
\hline NGC 2264 & $\approx 1$ & $\ldots$ & $\ldots$ & 30.4 & $\ldots$ & 4 \\
\hline Orion & $0.1-10$ & $\ldots$ & $\ldots$ & 29.8 & $\ldots$ & 5 \\
\hline NGC 2024 & 0.3 -few & $\ldots$ & $\ldots$ & $\ldots$ & $\ldots$ & 6 \\
\hline Taurus WTTS & $0.1-10$ & $\ldots$ & 30.2 & 29.9 & 29.1 & 7 \\
\hline ChaI WTTS & $0.1-10$ & $\ldots$ & $\ldots$ & 29.8 & $\ldots$ & 8 \\
\hline IC 348 & $1-6$ & $\ldots$ & $\ldots$ & 29.15 & $\ldots$ & 9 \\
\hline $\mathrm{R} \mathrm{CrA}$ & $1.5-7$ & $\ldots$ & $\ldots$ & $\ldots$ & $\ldots$ & 10 \\
\hline Upper Sco-Cen & $5-6$ & $\ldots$ & $\ldots$ & $\ldots$ & $\ldots$ & 11 \\
\hline$\eta$ Cha & 8 & $\ldots$ & $\ldots$ & $\ldots$ & $\ldots$ & 12 \\
\hline TW Hya & $10-30$ & $\ldots$ & $\ldots$ & 29.6 & $\ldots$ & 13 \\
\hline Tucanae & $10-30$ & $\ldots$ & $\ldots$ & 29.8 & $\ldots$ & 14 \\
\hline IC 2602 & 32 & $\ldots$ & 29.8 & 29.75 & 29.15 & 15 \\
\hline NGC 2547 & 36 & $\ldots$ & $\ldots$ & $\ldots$ & $\ldots$ & 16 \\
\hline IC 4665 & 43 & $\ldots$ & $\ldots$ & $\ldots$ & $\ldots$ & 17 \\
\hline IC 2391 & 46 & $\ldots$ & 29.75 & $\ldots$ & $\ldots$ & 18 \\
\hline$\alpha$ Per & 50 & 29.52 & 29.68 & 29.57 & 28.86 & 19 \\
\hline NGC $2451 \mathrm{~B}$ & 50 & 29.81 & 29.82 & 29.71 & $\ldots$ & 20 \\
\hline NGC $2451 \mathrm{~A}$ & $50-80$ & 29.23 & 29.46 & $\approx 29$ & 29.29 & 20 \\
\hline Blanco 1 & 63 & 29.45 & 29.41 & 29.11 & 29.06 & 21 \\
\hline NGC 2422 & 73 & $\ldots$ & $\approx 29.0$ & $\ldots$ & $\ldots$ & 22 \\
\hline Pleiades & 100 & 29.20 & 29.25 & 29.2 & 28.85 & 23 \\
\hline NGC 2516 & 110 & 29.40 & 29.16 & 29.08 & 28.72 & 24 \\
\hline Stock 2 & 170 & $\ldots$ & $\ldots$ & $\ldots$ & $\ldots$ & 25 \\
\hline NGC 1039 & 180 & $\ldots$ & $\ldots$ & $\ldots$ & $\ldots$ & 26 \\
\hline Ursa Major & 300 & $\approx 28.2$ & $\approx 28.2$ & $\ldots$ & $\ldots$ & 27 \\
\hline NGC 6475 & 300 & $(29.25)$ & (29.44) & (29.5) & $\ldots$ & 28 \\
\hline NGC 3532 & 310 & $\ldots$ & $\ldots$ & $\ldots$ & $\ldots$ & 29 \\
\hline NGC 6633 & 430 & $\approx 28.7$ & $\approx 28.5$ & $\ldots$ & $\ldots$ & 30 \\
\hline Coma Ber & 450 & 28.87 & $\ldots$ & $\ldots$ & $\ldots$ & 31 \\
\hline IC 4756 & 500 & $\ldots$ & $\ldots$ & $\ldots$ & $\ldots$ & 32 \\
\hline NGC 6940 & 720 & $\ldots$ & $\ldots$ & $\ldots$ & $\ldots$ & 33 \\
\hline Praesepe & 730 & $\ldots$ & 29.02 & 28.25 & 28.3 & 34 \\
\hline Hyades & 790 & 28.7 & 29.03 & 28.19 & 28.21 & 35 \\
\hline NGC 752 & 1100 & $\ldots$ & $\ldots$ & $\ldots$ & $\ldots$ & 36 \\
\hline IC 4651 & 1100 & $\ldots$ & $\ldots$ & $\ldots$ & $\ldots$ & 37 \\
\hline M67 & 2600 & $\ldots$ & $\ldots$ & $\ldots$ & $\ldots$ & 38 \\
\hline NGC 188 & 4300 & $\ldots$ & $\ldots$ & $\ldots$ & $\ldots$ & 39 \\
\hline
\end{tabular}

NOTE: For PMS clusters, the numbers refer to WTTS if separately available from the literature. If given as a class, they are listed in the K star column. ${ }^{\mathrm{a}}$ References and notes. Reference from which quoted values were taken are given in boldface. Approximate cluster ages are from the Lausanne open cluster database (WEBDA) or from the cited references. 1 Preibisch (1997b), Preibisch (2003a), Getman et al. (2002); 2 Montmerle et al. (1983), Koyama et al. (1994), Casanova et al. (1995), Kamata et al. (1997), Grosso et al. (2000), Grosso (2001), Imanishi et al. (2001a), Imanishi et al. (2001b), Imanishi et al. (2002), Imanishi et al. (2003); 3 Preibisch (1998), Preibisch (2003b); 4 Simon et al. (1985), Flaccomio et al. (1999), Flaccomio et al. (2000); 5 Gagné and Caillault (1994), Gagné et al. (1995b), Pravdo and Angelini (1995), Yamauchi et al. (1996), Garmire et al. (2000), Tsujimoto et al. (2002), Feigelson et al. (2002a), Feigelson et al. (2002b), Feigelson et al. (2003), Flaccomio et al. (2003a), Flaccomio et al. (2003b); 6 Freyberg and Schmitt (1995); Skinner et al. (2003); 7 Feigelson et al. (1987), Walter et al. (1988), Strom et al. (1990), Strom and Strom (1994), Damiani et al. (1995), Damiani and Micela (1995), Neuhäuser et al. (1995a), Carkner et al. (1996), Skinner et al. (1997), Stelzer and Neuhäuser (2001); 8 Feigelson and Kriss (1989), Feigelson et al. (1993), Lawson et al. (1996); 9 Preibisch et al. (1996), Preibisch and Zinnecker (2001), Preibisch and Zinnecker (2002); dominated by M dwarf sample; 10 Koyama et al. (1996), Walter et al. (1997); 11 Walter et al. (1994), Sciortino et al. (1998); 12 Mamajek et al. (1999), Mamajek et al. (2000); 13 Hoff et al. (1998), Jensen et al. (1998), Kastner et al. (1999), Stelzer and Neuhäuser (2000); 14 Stelzer and Neuhäuser (2000); 15 Randich et al. (1995); 16 Jeffries and Tolley (1998); 17 Giampapa et al. (1998); 18 Patten and Simon (1993), Patten and Simon (1996), Simon and Patten (1998); 19 Randich et al. (1996a), Prosser et al. (1996); 20 Hünsch et al. (2003), B biased toward too high $L_{X} ; 21$ Micela et al. (1999b), Pillitteri et al. (2003); 22 Barbera et al. (2002); 23 Caillault and Helfand (1985), Micela et al. (1985), Schmitt et al. (1993a), Stauffer et al. (1994), Gagné et al. (1995a), Hodgkin et al. (1995), Micela et al. (1996), Micela et al. (1999a), Stelzer and Neuhäuser (2001), Krishnamurthi et al. (2001), Daniel et al. (2002), Briggs and Pye (2003); 24 Dachs and Hummel (1996), Jeffries et al. (1997), Micela et al. (2000), Sciortino et al. (2001), Harnden et al. (2001), Damiani et al. (2003); 25 Sciortino et al. (2000); 26 Simon (2000); 27 Walter et al. (1984), Schmitt et al. (1990b); 28 Prosser et al. (1995), James and Jeffries (1997); sample is 
cluster shows distinctly lower median $L_{X}$ and $L_{X} / L_{\text {bol }}$ for most spectral classes, with the exception of $\mathrm{M}$ dwarfs. These systematics are clearly related to evolution and rotation (Caillault and Helfand 1985; Micela et al. 1985), but we now also see a strong dependence of the evolution on $B-V$ and therefore mass. Let us briefly walk across the available $B-V$ range.

As is the case for field stars (Sect. 4.4), A stars and early F-type stars are usually detected at very low luminosities or not at all, and in most other cases, there is a strong suspicion that later-type companions are responsible for the observed level of X-ray emission (Micela et al. 1985, 1988, 1996; Schmitt et al. 1990b; Stauffer et al. 1994; Randich et al. 1995, 1996a; Briggs and Pye 2003). F stars that are thought to be genuine Xray emitters not only fall short by one order of magnitude of the maximum $L_{X} / L_{\text {bol }}$ level attained by cooler stars, but their X-ray properties also show relatively little evolution in time, at least among younger clusters with ages up to a few $100 \mathrm{Myr}$ (Caillault and Helfand 1985; Micela et al. 1990; Patten and Simon 1996; Randich et al. 1996a). As discussed in Sect. 5, the decreasing X-ray efficiency toward earlier spectral types is most likely related to the decreasing depth of the convection zone and the consequent limited ability of the dynamo to cover the star with a strong magnetic corona.

The most luminous stars in young clusters are found among $G$ stars. These are the earliest stars that reach up to a saturation limit of $L_{X} / L_{\text {bol }} \approx 10^{-3}$ (Stauffer et al. 1994; Randich et al. 1996a, Fig. 40). At the same time, they also show the largest spread in Xray activity. An evolutionary decay in $L_{X}$ is well seen beyond the Pleiades' age: Hyades $\mathrm{G}$ stars are no longer found at the saturation level. The X-ray activity evolution of $\mathrm{G}$ stars thus proceeds on time scales of $\approx 100 \mathrm{Myr}$.

Moving now to $\mathrm{K}$ dwarfs, similar systematics are seen except that the upper bound of $L_{X}$ continuously drops toward later spectral types (Fig. 40), which is a direct consequence of the decreasing maximum $L_{X}$ allowed by the saturation boundary $L_{X} / L_{\mathrm{bol}} \approx$ $10^{-3}$. The evolution seems to be slowest among $M$ dwarfs that are still close to the saturation level in the Hyades. As already mentioned above for field stars, the slower spin-down keeps their magnetic activity at high levels during at least several $100 \mathrm{Myr}$.

\subsubsection{Rotation-age-activity relations}

Open clusters offer a great amount of information on rotation-activity and activityage relations. Although initial reports failed to find such dependencies (Caillault and Helfand 1985; Micela et al. 1985), the best-defined rotation-activity relations in fact now derive from open cluster studies and include the saturation plateau and stars in the supersaturation regime (Randich et al. 2000, Fig. 4).

Such investigations have converged to a comprehensive basic picture of MS coronal evolution (e.g., Stern et al. 1995b; Patten and Simon 1996). As the stars arrive on the main sequence, their initial rotation periods may largely vary, probably as a consequence of

X-ray selected - true median should be lower; 29 Simon (2000), Franciosini et al. (2000); 30 Briggs et al. (2000), Harmer et al. (2001); upper limit to median given; 31 Randich et al. (1996b); 32 Randich et al. (1998), Briggs et al. (2000); 33 Belloni and Tagliaferri (1997); 34 Randich and Schmitt (1995), Randich et al. (1996b), Barrado Y Navascués et al. (1998), Franciosini et al. (2004); 35 Stern et al. (1981), Micela et al. (1988), Stern et al. (1992c), Stern et al. (1994), Pye et al. (1994), Stern et al. (1995b), Reid et al. (1995), Stelzer and Neuhäuser (2001), G. Micela, priv. comm. (2004); 36 Belloni and Verbunt (1996); 37 Belloni and Tagliaferri (1998); 38 Belloni et al. (1993), Belloni et al. (1998); 39 Belloni et al. (1998). 


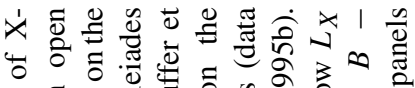

。ี $\cong$ 造壳

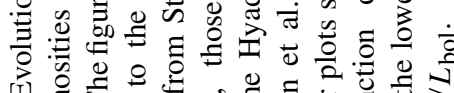

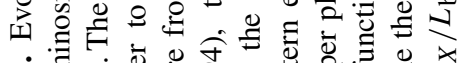

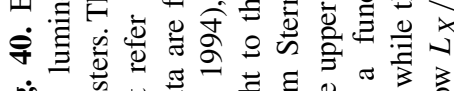

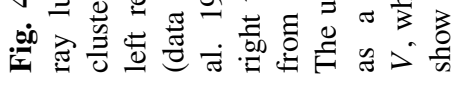
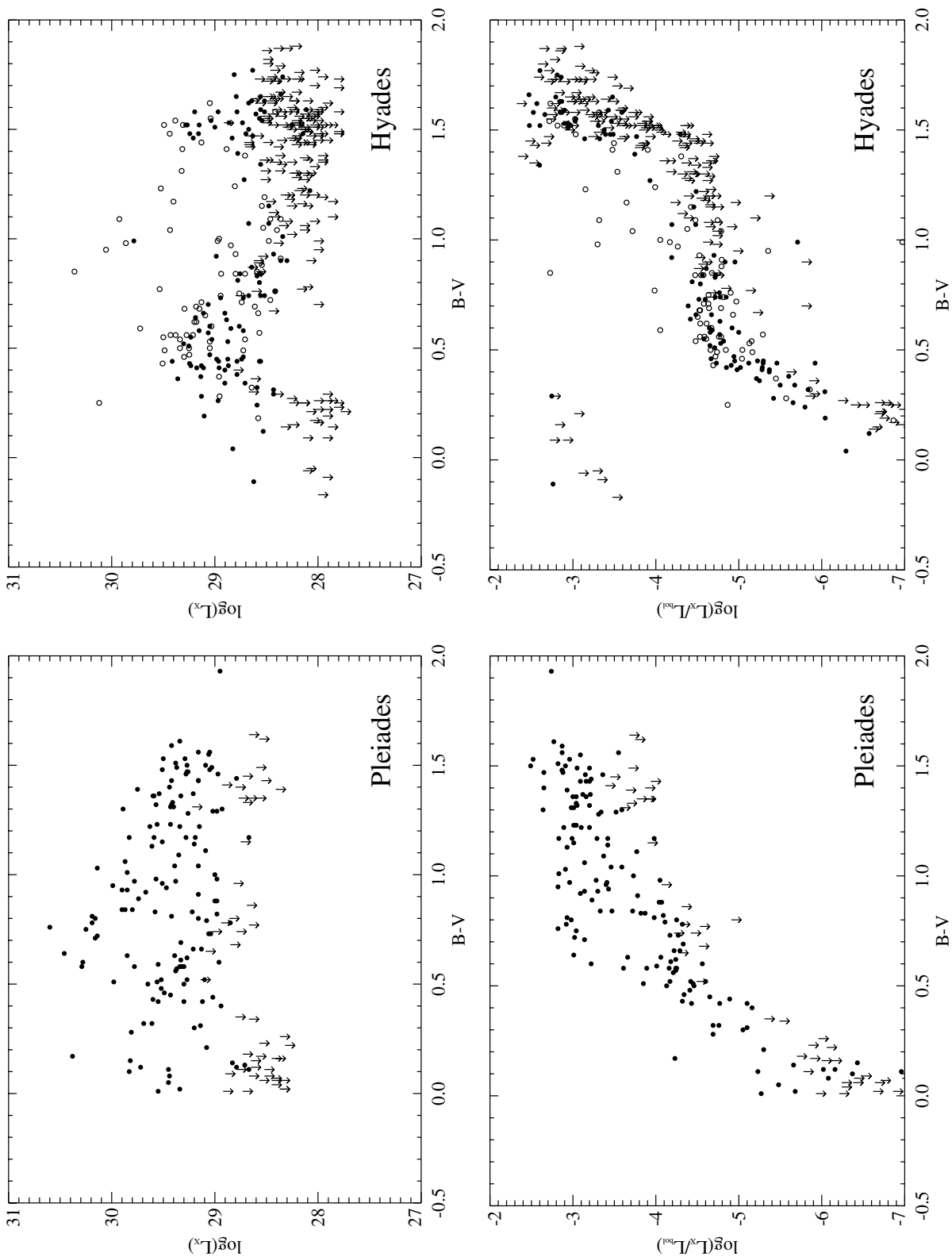
different star-disk coupling and disk-dispersal histories. Somewhat counter-intuitively, X-ray luminosities remain narrowly confined at predictable levels during this phase. This is because most stars rotate rapidly enough to keep them in the saturation regime where $L_{X}$ is controlled essentially by $L_{\text {bol }}$ rather than by rotation. Stars thus typically enter the rotation-activity diagram (Fig. 4) spread over a range in the saturation regime. There is often some additional intrinsic scatter that is possibly related to long-term, cyclic modulation (Patten and Simon 1996, but see Micela et al. 1996; Simon and Patten 1998).

The rotation rate of stars declines steadily as they lose angular momentum via a magnetized wind. Magnetic braking occurs at the highest rate for the most rapid rotators, because they produce the strongest magnetic fields and presumably winds. The stars eventually drop out of the saturation regime and enter the standard rotation-activity branch, but not all at the same time given their spread of initial rotation periods. This is probably the cause of the wide spread seen in $P$ and $L_{X}$ below the saturation regime at any given moment in time, i.e., for a given cluster, as long as the cluster is relatively young (Stauffer et al. 1994).

Because the spin-down rate is higher for higher-mass stars, the X-ray activity of the earliest-type stars decays first. This leads to the characteristic development of the $L_{X}$ vs. $B-V$ diagram where the G-K regime is "hollowed out" from the left on times scales of $20-50 \mathrm{Myr}$ for G stars and $\gtrsim 75 \mathrm{Myr}$ to a few $100 \mathrm{Myr}$ for K stars, while M dwarfs remain at high activity levels for much longer (Patten and Simon 1996; James and Jeffries 1997, Fig. 40). Incidentally, the distribution of $L_{X}$ levels may then be relatively flat across a wide range of $B-V$ in the age period between the Pleiades and the Hyades. At the same time, the $L_{X} / L_{\text {bol }}$ ratio systematically increases toward larger $B-V$ (Fig. 40). At the age of $\alpha$ Per or the Pleiades, G, K, and M dwarfs still all reach up to the saturation limit (Hodgkin et al. 1995; Prosser et al. 1996; Randich et al. 1996a; Micela et al. 1999a), while at the ages of the Hyades, this is true only for late-K and M dwarfs (Reid et al. 1995; Stern et al. 1995b). The break point at which saturation is reached thus moves to progressively larger $B-V$ as the cluster ages.

The different braking histories lead to different characteristic decays of the X-ray luminosities for various spectral ranges, as illustrated in Fig. 41. The error bars refer to the $\pm 1 \sigma$ spread read off the luminosity functions published in the literature. They are only approximate and are probably dominated by uncertainties from small-number statistics. As we see from Fig. 41, the $L_{X}$ of all spectral classes decays beyond $100 \mathrm{Myr}$ although this decay is clearly slowest for M dwarfs. As a cluster reaches the Hyades' age $(\approx 700-800 \mathrm{Myr})$, the rotation periods of both $\mathrm{G}$ and $\mathrm{K}$ dwarfs have mostly converged to relatively low values with little spread, and the $L_{X}$ values are consequently also expected to have dropped significantly below the saturation limit, with small statistical spread, while $\mathrm{M}$ dwarfs are now in a regime of rapid braking, increasing their scatter in $L_{X}$ as they settle at lower rotation rates (Micela et al. 1988; Stern et al. 1992c, 1994, 1995b).

The overall trend agrees nicely with the decay law found from field stars, as is illustrated for the $\mathrm{G}$ star panel where the field stars from Fig. 38 are overplotted as filled circles. This scenario is alternatively illustrated by X-ray luminosity functions for various spectral ranges; the sample median and the spread of X-ray luminosities as a 

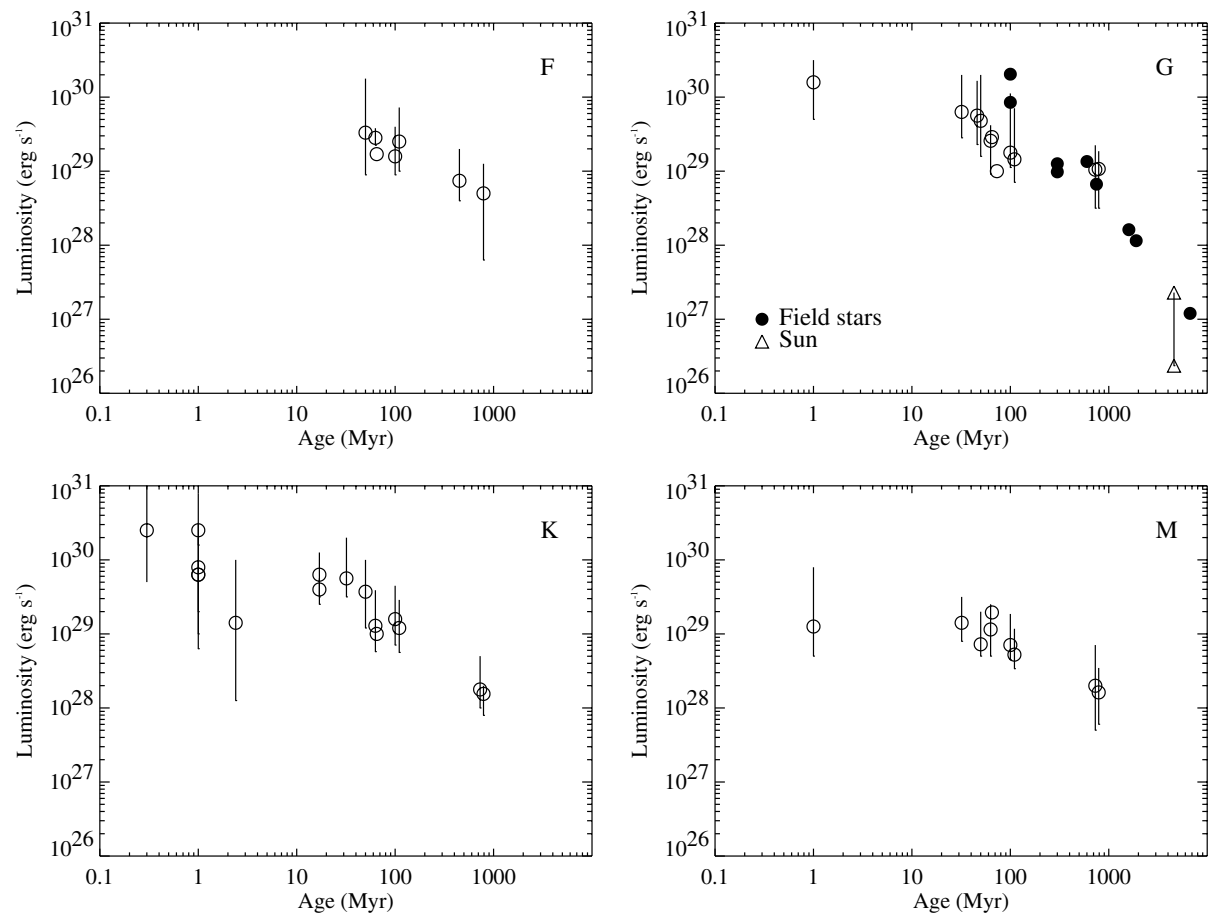

Fig. 41. The evolution of the median X-ray luminosities of F, G, K, and M stars derived from various open clusters (from Table 7: $\rho$ Oph, NGC 2264, Orion, Taurus, Cha I, IC 348, TW Hya, Tucanae, IC 2602, IC 2391, $\alpha$ Per, Blanco 1, NGC 2451 A, NGC 2422, Pleiades, NGC 2516, Coma Ber, Praesepe, and Hyades). The error bars give the approximate $1 \sigma$ scatter (if available from the literature). The panel for G stars also includes the field G stars shown in Fig. 38 (filled circles). The TTS samples from star-forming regions are lumped together in the K star panel, except for the Taurus region for which $\mathrm{G}, \mathrm{K}$, and $\mathrm{M}$ stars are given separately. The ages of the star-forming regions are only characteristic averages as they are typically spread between $0.1-10 \mathrm{Myr}$

function of spectral type can then be followed as a function of age (e.g., Micela et al. 1990), as shown in Fig. 42.

At this point, we recognize the close interrelationship between the activity-age relationship discussed here (see also Sect. 17.1 and (61)), the temperature-activity relation introduced in Sect. 9.5 (Fig. 10, (16)), and the temperature-age relation (Sect. 17.1, Fig. 38). They are all an expression of the coupling between coronal activity and the rotation-induced internal dynamo.

\subsubsection{Binaries}

A different scenario seems to apply to late-type binary stars even if they are not tidally locked. Pye et al. (1994) and Stern et al. (1995b) found components in K- and M-type binaries (but not F- and G-type binaries) in the Hyades to be overluminous by an order of magnitude when compared to single stars. The explanation for this effect is unclear but could involve a less rapid braking in the PMS stage as the circumstellar disks were 

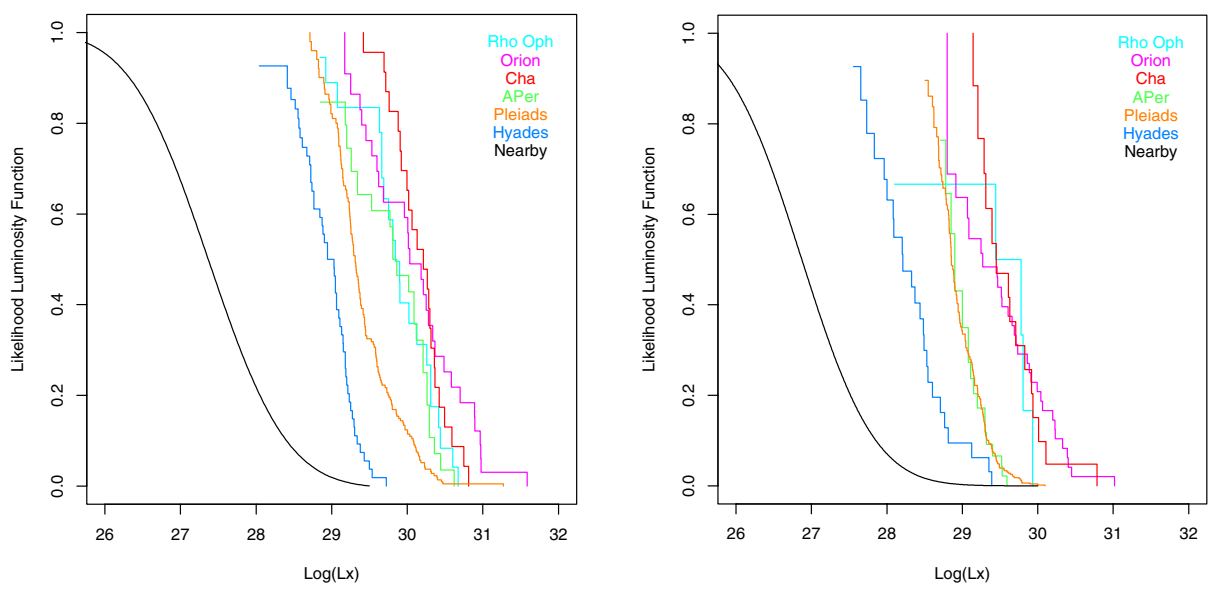

Fig. 42. Luminosity functions of (left) stars with masses of $\approx 0.5-1 M_{\odot}$, representative of G-K stars, and (right) with masses of $\approx 0.25-0.5 M_{\odot}$, representative of M-type stars, for various starforming regions and open clusters. The median luminosity decreases with increasing age (figures courtesy of G. Micela; references used: Pye et al. 1994; Stern et al. 1995b; Randich et al. 1996a; Flaccomio et al. 2003c)

disrupted, thus producing very rapid rotators that have still not spun down at the age of the Hyades (Pye et al. 1994). A reanalysis by Stelzer and Neuhäuser (2001), however, questioned a significant difference between singles and binaries.

\subsubsection{Co-eval clusters}

Intercomparisons between clusters of the same age have greatly helped confirm the above evolutionary picture. Similar trends for $L_{X}$ and $L_{X} / L_{\text {bol }}$ are generally found for co-eval clusters (e.g., Giampapa et al. 1998). A notable exception was discussed by Randich and Schmitt (1995), Randich et al. (1996b), and Barrado Y Navascués et al. (1998) who compared the nearly co-eval Hyades, Coma Berenices, and Praesepe clusters, finding significant $L_{X}$ deficiencies in the latter cluster but agreement between the former two. The cause of the discrepancy is not clear, but could be related to the recent finding that Praesepe shows a spatial segregation of activity levels, which may be the result of a merger of two non-coeval clusters (Franciosini et al. 2004 and references therein). Similar discrepant cases include Stock 2 (Sciortino et al. 2000) and possibly IC 4756 (Randich et al. 1998), NGC 3532 (Franciosini et al. 2000), and NGC 6633 (Briggs et al. 2000; Harmer et al. 2001). Another important influence may come from different metallicities in clusters. Jeffries et al. (1997), Harnden et al. (2001), Sciortino et al. (2001), and Damiani et al. (2003) compared NGC 2516 with the slightly younger Pleiades and found F stars to be more luminous and G/K stars less luminous in the former. Jeffries et al. (1997) suspected that the rotational history of the F stars is different owing to different convective turnover times given NGC 2516's lower metallicity. This explanation, however, seems to be ruled out by recent spectroscopy that indicates solar metallicity for this cluster. Slightly different rotation period distributions may be present for $\mathrm{G}$ and $\mathrm{K}$ stars perhaps due to a small age difference (see Damiani et al. 2003 and references therein). A metallicity- 
related effect on X-ray radiation has also been discussed for the metal-rich cluster Blanco 1 that reveals an X-ray excess for M dwarfs (Micela et al. 1999b; Pillitteri et al. 2003).

\subsubsection{Toward older clusters}

An extension of cluster studies toward higher ages suffers both from the small number of clusters that are still bound, thus implying larger typical distances, and from the low $\mathrm{X}$-ray luminosities of old main-sequence stars. Only close binaries that are kept in rapid rotation by tidal forces, or giants have typically been detected in such clusters, with no less interesting results (e.g., Belloni and Verbunt 1996, Belloni and Tagliaferri 1997, and Belloni et al. 1998 for binaries in NGC 752, NGC 6940, and M 67, respectively; see Table 7).

\subsubsection{Toward younger clusters}

Clusters significantly younger than $\alpha$ Per connect to pre-main-sequence (PMS) evolution and the phase of circumstellar-disk dispersal. For example, Jeffries and Tolley (1998) found the X-ray luminosities of all $\mathrm{G}$ and $\mathrm{K}$ stars in the young (14 Myr; $\approx 35 \mathrm{Myr}$ according to other sources) cluster NGC 2547 to be a factor of two below the saturation limit of $L_{X} / L_{\text {bol }} \approx 10^{-3}$, suggesting that all stars have rotation periods $>3 \mathrm{~d}$. Possibly, their circumstellar disks have only recently, at an unusually late point in time, been dispersed and the stars have not yet spun up from the disk-controlled slow rotation. The preceding evolution of disk environments is thus obviously of pivotal importance for cluster development, as we will discuss in the next section.

\section{X-ray coronae and star formation}

Modern theory of star formation together with results from comprehensive observing programs have converged to a picture in which a forming low-mass star evolves through various stages with progressive clearing of a contracting circumstellar envelope. In its "class 0" stage (according to the $\mathrm{mm} / \mathrm{infrared}$ classification scheme), the majority of the future mass of the star still resides in the contracting molecular envelope. "Class I" protostars have essentially accreted their final mass while still being deeply embedded in an envelope and surrounded by a thick circumstellar disk. Jets and outflows may be driven by these optically invisible "infrared stars". Once the envelope is dispersed, the stars enter their "classical T Tauri" stage (CTTS, usually belonging to class II) with excess $\mathrm{H} \alpha$ line emission if they are still surrounded by a massive circumstellar disk; the latter results in an infrared excess. "Weak-lined T Tauri stars" (also "naked T Tauri stars", Walter 1986; usually with class III characteristics) have lost most of their disk and are dominated by photospheric light (Walter et al. 1988).

Consequently, X-ray emission from these latter stars has unequivocally been attributed to solar-like coronal activity (Feigelson and DeCampli 1981; Feigelson and Kriss 1981, 1989; Walter and Kuhi 1984; Walter et al. 1988), an assertion that is less clear for earlier PMS classes. Arguments in favor of solar-like coronal activity in all TTS include i) typical electron temperatures of order $10^{7} \mathrm{~K}$ that require magnetic confinement, ii) a "solar-like" correlation with chromospheric emission, iii) the presence of 
flares, and iv) rotation-activity relations that are similar to those in more evolved active stars (e.g., Feigelson and DeCampli 1981; Walter and Kuhi 1984; Walter et al. 1988; Bouvier 1990; Damiani and Micela 1995). The solar analogy may, on the other hand, not hold for the emission excess of optical lines in CTTS; this excess is uncorrelated with X-rays (Bouvier 1990) but that seems to relate to the accretion process.

Because this review is primarily concerned with coronal X-rays that are - in the widest sense - solar-like, the discussions in the following sections are confined to lowmass stars in nearby star-forming regions. For a broader view of X-rays in the starformation process, I refer the reader to the review by Feigelson and Montmerle (1999) and references therein.

\subsection{T Tauri stars}

\subsubsection{Overview}

Because most of the low-mass CTTS and WTTS are fully convective, there has been a special interest in their X-ray behavior and in their rotation-activity relations. Quite some debate has unfolded on whether CTTS and WTTS belong to the same parent population if their X-ray luminosities are compared. CTTS have been found to be, on average, less luminous than WTTS in particular in Taurus (Strom and Strom 1994; Damiani et al. 1995; Neuhäuser et al. 1995a; Stelzer and Neuhäuser 2001), but other authors have found the two samples to be indistinguishable in various other star-forming regions (Feigelson and Kriss 1989; Feigelson et al. 1993; Strom et al. 1990; Casanova et al. 1995; Lawson et al. 1996; Grosso et al. 2000; Flaccomio et al. 2000; Preibisch and Zinnecker 2001, 2002; Feigelson et al. 2002a; Getman et al. 2002, but see contradicting result for Orion by Flaccomio et al. 2003b).

It is plausible that WTTS are stronger X-ray sources than CTTS because the latters' X-ray emission could be absorbed by an overlying wind (Walter and Kuhi 1981, see also Stassun et al. 2004), their coronal activity could somehow be inhibited by the process of mass accretion onto the stellar surface (Damiani and Micela 1995), or because WTTS produce more efficient dynamos given their generally higher measured rotation rates (Neuhäuser et al. 1995a), although most PMS are in the saturation regime (Flaccomio et al. 2003b). However, there are also a number of arguments against any intrinsic Xray difference between WTTS and CTTS. CTTS are usually identified optically, while WTTS are inconspicuous at those wavelengths but are typically selected from X-ray surveys (Feigelson et al. 1987) where many of them stand out given their rapid rotation, hence their bias toward strong X-rays (Feigelson and Kriss 1989; Preibisch et al. 1996). Several authors (Gagné and Caillault 1994; Damiani et al. 1995; Preibisch and Zinnecker 2001, 2002; Getman et al. 2002; Feigelson et al. 2002a, 2003) also tested X-ray activity against infrared excess, but no distinction was found between the two classes, suggesting that the observed X-ray emission does not directly relate to the presence of massive disks.

This issue has seen some, albeit still not full, clarification with recent work by Flaccomio et al. (2003a,b,d) who studied various ranges of stellar mass. It probably does matter whether stars are distinguished by an indicator for the presence of a disk (such as their IR classification) or by an indicator of active accretion (e.g., $\mathrm{H} \alpha$ emission). There is no one-to-one correspondence between these indicators (see also Preibisch and 


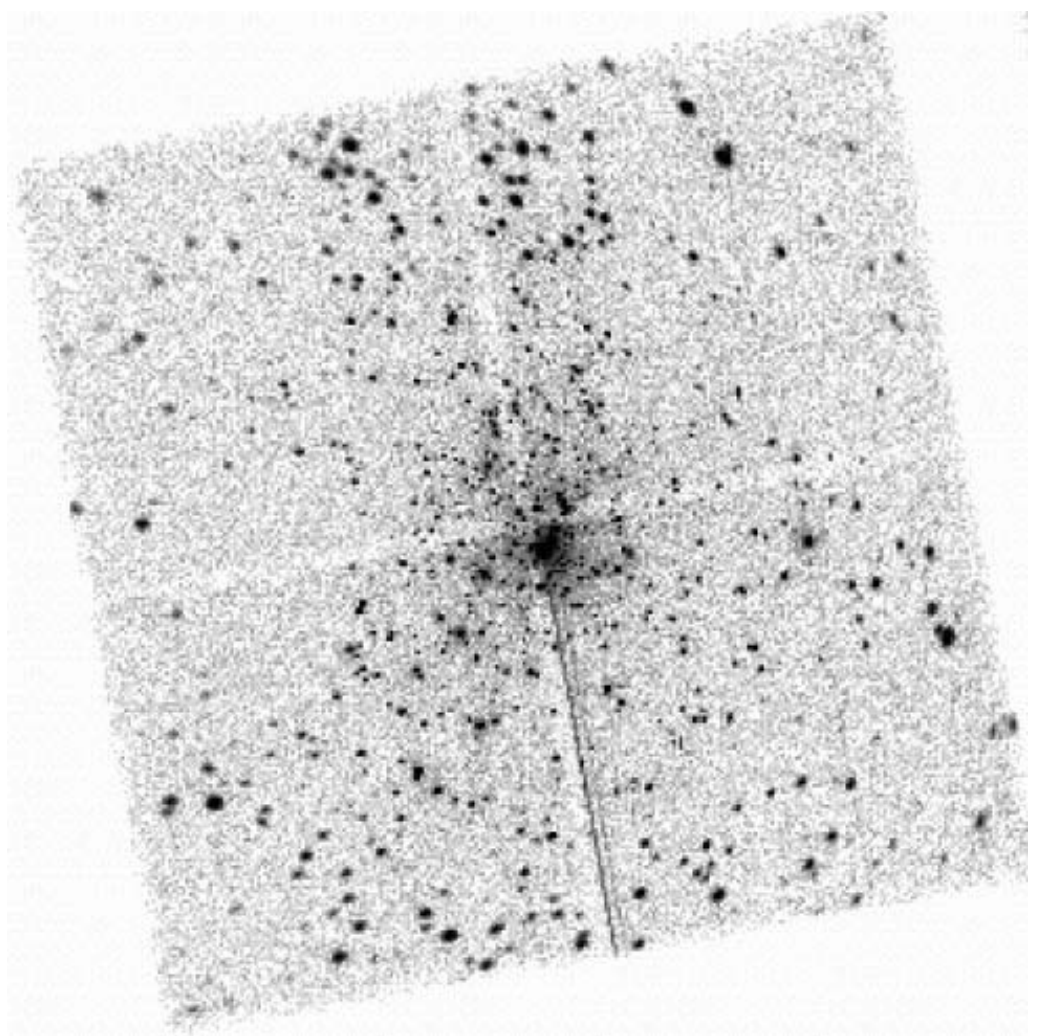

Fig. 43. The Orion Nebula Cluster as seen in X-rays by Chandra. North is up (Feigelson et al. 2002a)

Zinnecker 2002). The debate remains open; Flaccomio et al.'s work seems to suggest that either indicator points at CTTS being less X-ray luminous than WTTS in a given mass range, at least for the considered star-forming clusters. Stassun et al. (2004) argued, from a reconsideration of the Orion X-ray samples, that the suppression of X-ray luminosity in a subsample of stars, accompanied by increased hardness, is in fact only apparent and is due to the increased attenuation of softer photons by magnetospheric accretion columns in actively accreting stars.

\subsubsection{X-ray luminosity and age}

Spectroscopic evidence and EM distributions in TTS point to an analogy to young solar analogs. It appears that both the EM and the hot temperatures are progressively more enhanced as one moves from the ZAMS into the pre-main sequence regime of CTTS (Fig. 44, Skinner and Walter 1998). The overall X-ray levels of pre-main sequence TTS also fit into the general picture of declining X-ray activity with increasing age (Walter et al. 1988; Feigelson and Kriss 1989; Feigelson et al. 1993; Gagné and Caillault 1994; Gagné et al. 1995b; Damiani et al. 1995; Stelzer and Neuhäuser 2001, Figs. 41, 42), although this effect is not directly - or not only - coupled with rotation. While WTTS 


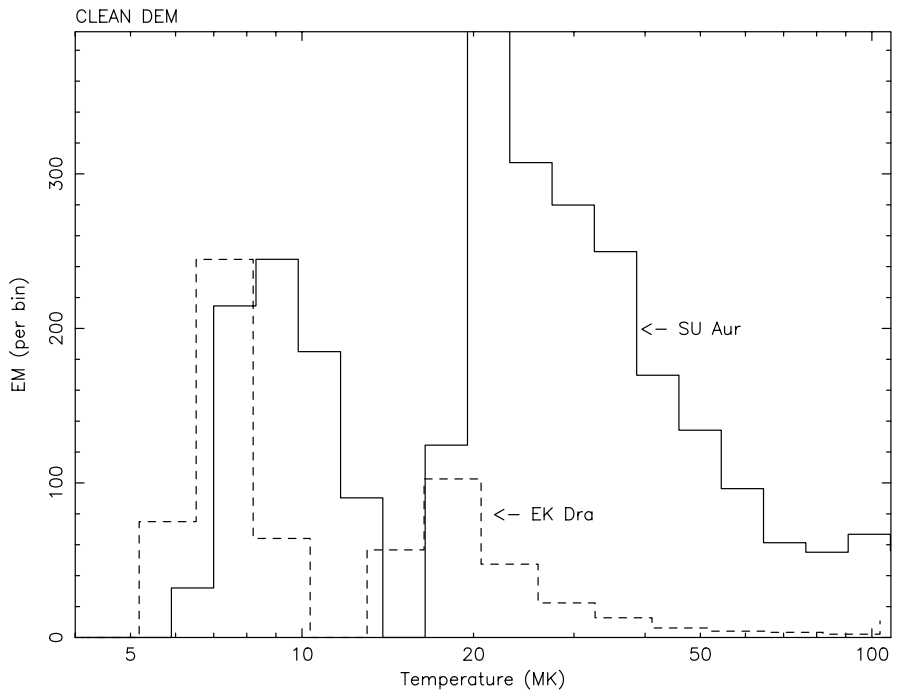

Fig. 44. Comparison of EMDs of the CTTS SU Aur (solid histogram) and the ZAMS solar analog EK Dra (dashed histogram; figure courtesy of S. Skinner, after Skinner and Walter 1998)

may spin up toward the main sequence and reach X-ray saturation, they at the same time contract and decrease in $L_{\text {bol }}$; the X-ray luminosity then peaks around an age of 1 Myr and subsequently slowly decays (Neuhäuser et al. 1995a; Damiani and Micela 1995; Feigelson et al. 2003; Flaccomio et al. 2003c) whereas $L_{X} / L_{\text {bol }}$ remains saturated for all TTS during their descent along the Hayashi track (Flaccomio et al. 2003b,a). It is important to mention that CTTS and WTTS properties do not provide reliable age indicators per se. CTTS and WTTS in a star-forming region may have the same age while disk/envelope dispersal histories were different, although WTTS do tend to dominate the final pre-main sequence episodes (see, e.g., Walter et al. 1988; Feigelson et al. 1993; Lawson et al. 1996; Alcalá et al. 1997; Stelzer and Neuhäuser 2001).

\subsubsection{X-ray luminosity, saturation, and rotation}

Rotation may therefore be a more pivotal parameter. In this context, activity-rotation relationships are particularly interesting because the rotation history is strongly coupled with the presence of accretion disks, probably by magnetic coupling. CTTS generally rotate slowly, with rotation periods typically of $P \approx 4-8$ days; only once the stars have lost their massive inner accretion disks will the star spin up to rotation periods of typically one to a few days (Bouvier et al. 1993). Many T Tau stars, in particular WTTS, are therefore in the saturation regime (Bouvier 1990; Strom et al. 1990; Strom and Strom 1994; Gagné and Caillault 1994; Gagné et al. 1995b; Casanova et al. 1995; Flaccomio et al. 2000). While often quoted as supportive of the same type of magnetic activity as in normal stars, this result is, at hindsight, rather surprising because PMS have no significant, stable nuclear energy source. The saturation level therefore does not seem to relate to the total nuclear energy production. 
A normal rotation dependence of $L_{X}$ can be found as well, in particular in the Taurus sample. In this case, excellent agreement is found with the behavior of more evolved stars, again suggesting that essentially the same type of magnetic dynamo and coronal activity are at work (Bouvier 1990; Damiani and Micela 1995; Neuhäuser et al. 1995a; Stelzer and Neuhäuser 2001). However, in other star-forming regions, such as the Orion Nebula Cluster, $L_{X}$ appears to be independent of $P_{\text {rot }}$ up to quite long periods of $30 \mathrm{~d}$, the stars essentially all residing in a saturated regime despite considerable scatter in $L_{X} / L_{\text {bol }}$.

Feigelson et al. (2003) discussed these results in terms of various dynamo theories. On the other hand, Flaccomio et al. (2003c) have studied rotation and convection for various PMS age ranges. Convection zone parameters indicate that most PMS stars should indeed be in the saturation regime, as observed, for example, in the Orion Nebula Cluster. The interesting point is, however, that the very youngest stars show a suppressed "saturation" level, by as much as an order of magnitude (e.g., $L_{X} / L_{\text {bol }} \approx 10^{-4}$ for Orion and $\rho$ Oph, Feigelson et al. 2003; Skinner et al. 2003; Grosso et al. 2000). Whereas Feigelson et al. (2003) hypothesized that a less efficient distributed dynamo is in effect, Flaccomio et al. (2003c) speculated that the disk is somehow inhibiting strong coronal activity during the first few Myr, which then led them to find a characteristic (inner-) disk dispersal time of 1-2 Myr.

\subsubsection{The widely dispersed "field WTTS" samples}

The easy identification of WTTS in X-ray surveys has led to a rather controversial issue related to the dispersal of star-formation clusters and TTS evolution. In general, the location of TTS relative to the cloud cores provides interesting insight into the star formation history in and around a molecular cloud. Feigelson et al. (1987), Walter et al. (1988), Feigelson and Kriss (1989), and Strom et al. (1990) were first to point to a rather large overpopulation of WTTS in Taurus by factors of 2 to 10 compared with CTTS, probably constituting the long-sought post-T Tau population evolving toward the ZAMS. An estimated WTTS/CTTS ratio of order 10 is then consistent with a disk dispersal time of a few Myr (Neuhäuser et al. 1995c; Feigelson 1996). If star formation is cut off in time, the ratios may even be higher; Walter et al. (1994) found a ratio of 40 in Upper Sco-Cen where star formation has ceased about $2 \mathrm{Myr}$ ago.

Nevertheless, it came as quite a surprise when further associated WTTS were discovered tens of degrees away from the Taurus, Chamaeleon, Orion, and Lupus molecular clouds (Walter et al. 1988; Neuhäuser et al. 1995b; Sterzik et al. 1995; Wichmann et al. 1996; Alcalá et al. 1995, 1997; Krautter et al. 1997). They show no spatial correlation with the present molecular clouds, which suggests that they have either drifted away from their place of formation (Neuhäuser et al. 1995b), or that they are the products of star formation in now dispersed molecular clouds (Walter et al. 1988) or local high-velocity, turbulent cloudlets (Feigelson 1996). The latter explanation is attractive because some of the samples are as young as $10^{6} \mathrm{yr}$ despite their large distance from the (present-day) "parent cloud" (Alcalá et al. 1997).

However, the evolutionary stage of these samples has been the subject of considerable debate. On the one hand, Neuhäuser et al. (1997) estimated their ages at typically $\leq$ $30 \mathrm{Myr}$, including a moderately-sized population of widely distributed ZAMS G stars. 
On the other hand, Micela et al. (1993), Briceño et al. (1997), and Briceño et al. (1999) used star-count models, statistics of X-ray detected M dwarfs, and Li measurements to argue that the dispersed WTTS do not relate to the "missing post-T Tau stars" but mostly have a larger, near-ZAMS age of $20-100 \mathrm{Myr}$. This would agree with the nearZAMS status of X-ray selected field stars reported by Micela et al. (1997b) that were, however, not drawn from the sample in question. Such an age population would also solve the problem of the "missing" ZAMS population that is expected from standard star formation models. A likely explanation for the potential misclassification of young field stars as WTTS may relate to the use of low-resolution spectroscopy for Li measurements (Briceño et al. 1997; Favata et al. 1997d; Micela et al. 1997b; Martín and Magazzù 1999). If this revised age classification is correct, then, however, the problem with the "missing post-T Tau" stars at younger ages of $2-10$ Myr reopens.

Covino et al. (1997), Alcalá et al. (1998), and Alcalá et al. (2000) reconsidered and discussed this issue for the Chamaeleon and the Orion regions, arriving at intermediate conclusions. The distributed "WTTS" population appears to consist of at least 50\% genuinely young ( $<5 \mathrm{Myr}$ ) WTTS somewhat concentrated toward the molecular clouds, plus a smaller, widely distributed population of unrelated older, possibly near-ZAMS field stars. There is little evidence for a genuine post-T Tau population, indicating that the star formation process in a given cloud occurs on time scales $<10 \mathrm{Myr}$ and is not continuous, as assumed in some of the population models (see also discussion in Favata et al. 1997d and the counter-arguments in Feigelson 1996). The entire issue remains under debate; see, for example, Alcalá et al. (2000) and references therein for a recent assessment discussing Li abundance, the location of the stars on the HRD, and their spatial distribution.

An argument supporting an intermediate age of a few $10^{7} \mathrm{yrs}$ for these stars at the interface between PMS and ZAMS may be their large-scale spatial correlation with the young Gould Belt structure which also contains several star formation regions in the solar vicinity (Krautter et al. 1997; Wichmann et al. 1997). The ages of many foreground Orion "WTTS" are indeed compatible with this hypothesis (Alcalá et al. 1998) - see Sect. 19.

\subsubsection{Flares}

X-ray flares have given clear evidence for underlying magnetic fields not only in WTTS (Walter and Kuhi 1984) but also in CTTS (Feigelson and DeCampli 1981; Walter and Kuhi 1984). They strongly support the presence of solar-like closed coronae in the broadest sense. From an energetics point of view, they play a very important role: As much as half of the emitted X-ray energy, if not more, may be due to strong flares (Montmerle et al. 1983), and many TTS are nearly continuously variable probably also owing to flares (Mamajek et al. 2000; Feigelson et al. 2002a; Preibisch and Zinnecker 2002; Skinner et al. 2003). Examples with extreme luminosities and temperatures up to $100 \mathrm{MK}$ have been reported (Feigelson and DeCampli 1981; Montmerle et al. 1983; Preibisch et al. 1995; Skinner et al. 1997; Tsuboi et al. 1998, 2000; Imanishi et al. 2002, see Sect. 12). Stelzer et al. (2000) systematically studied flares in TTS, comparing them with flares in the Pleiades and the Hyades clusters. They found that TTS flares tend to reach higher luminosities and temperatures than their main-sequence equivalents, and 
the flare rate above a given limit is also higher. The most extreme flares are found on CTTS, a possible hint at star-disk magnetic interactions during flares although this is at variance with suggestions made by Montmerle et al. (2000) (Sect. 18.2.2). The high activity level found for CTTS is echoed by the work of Skinner et al. (2003) who noted a large variability fraction among the hottest and most absorbed sources in NGC 2024.

\subsubsection{The circumstellar environment}

The high-energy emission related to the luminous X-rays may have considerable impact on the circumstellar environment and on the entire surrounding molecular clouds because it changes the ionization balance and induces chemical reactions in molecular material (Montmerle et al. 1983; Casanova et al. 1995; Kastner et al. 1999). Recently, Feigelson et al. (2002b) speculated that the elevated rate of large flares in young solar analogs in the Orion Nebula Cluster may be applicable to the young Sun. The increased activity may possibly explain the production of chondrules by flash-melting and isotopic anomalies in meteorites by high proton fluxes.

\subsubsection{Accretion-driven X-ray emission?}

An entirely different model for X-ray production in CTTS was put forward by Kastner et al. (2002) based on observations of the CTTS TW Hya. This star has been conspicuous by producing luminous radiation with $L_{X}=2 \times 10^{30} \mathrm{erg} \mathrm{s}^{-1}$ which is, however, very soft, with a best-fit temperature of $T \approx 3$ MK (Hoff et al. 1998; Kastner et al. 1999). While unusual for active coronal sources, the temperature is compatible with shockinduced X-ray emission at the base of magnetically funneled accretion flows. Explicit density measurements using He-like triplets of O vII and Ne IX indeed suggest very high densities of $n_{e}=10^{12}-10^{13} \mathrm{~cm}^{-3}$, densities that are not seen at these temperatures in any coronal source. Stelzer and Schmitt (2004) have supported this view and further argued that the low $\mathrm{C}$ and $\mathrm{Fe}$ abundances relate to their being grain-forming elements, that is, $\mathrm{C}$ and $\mathrm{Fe}$ have condensed out in the circumstellar disk or cloud. In the light of similarly extreme abundance anomalies in other stars (Sect. 16), this model necessarily remains tentative at this time.

The role of accretion columns for the X-ray production has further been discussed by Stassun et al. (2004). They found that actively accreting stars in the Orion Nebula Cluster on average show less luminous but also harder X-ray emission than non-accreting stars, suggesting that accretion columns may attenuate the X-rays while the intrinsic X-ray emission is similar in both samples, i.e., not related to accretion but to rotation. A timedependent effect of this sort has possibly been seen in the CTTS XZ Tau (Favata et al. 2003). To what extent TW Hya is an exception presently remains open.

\subsection{Protostars}

\subsubsection{Overview}

X-rays from embedded ("infrared") protostars are strongly attenuated and require access to relatively hard photons. The protostellar survey by Carkner et al. (1998) listed only about a dozen X-ray class I protostars detected at that time, with luminosities of order 

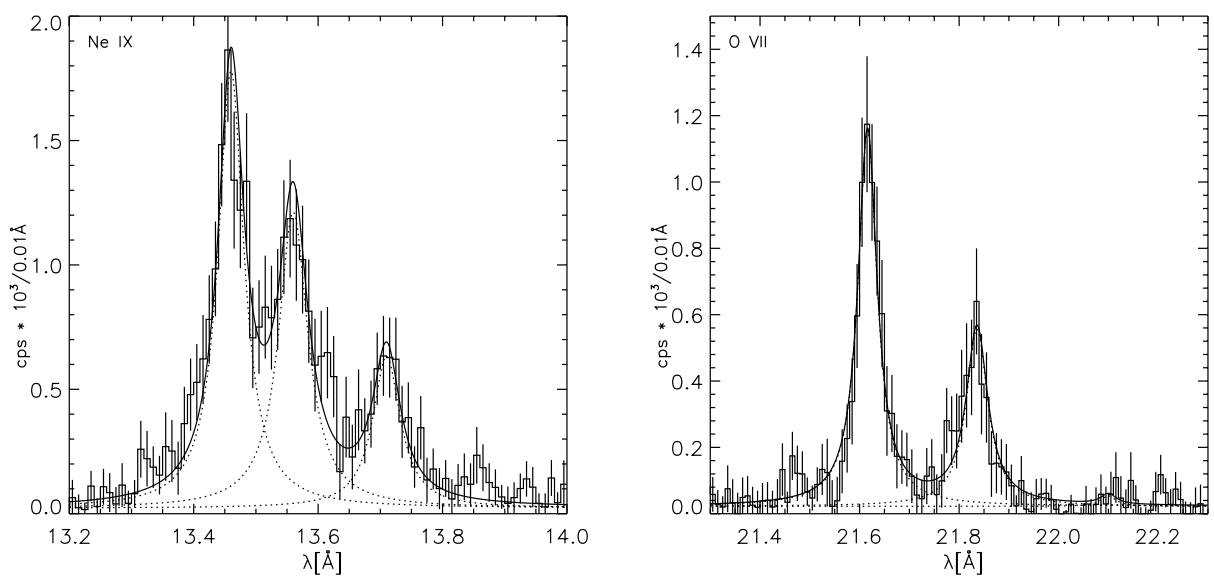

Fig. 45. He-like Ne IX (left) and O viI triplets (right) of the nearby CTTS TW Hya observed by XMM-Newton. The forbidden lines at $13.7 \AA$ and $22.1 \AA$, respectively, are largely suppressed, indicating high electron densities (figures courtesy of B. Stelzer, after Stelzer and Schmitt 2004)

$10^{30} \mathrm{erg} \mathrm{s}^{-1}$, and no class 0 objects. First tentative evidence for heavily absorbed hard emission was reported for $\rho$ Oph (Koyama et al. 1994; Casanova et al. 1995), followed by several unambiguous detections of very hard class I sources with temperatures of up to $7 \mathrm{keV}$ in the R CrA cloud (Koyama et al. 1996; Neuhäuser and Preibisch 1997), $\rho$ Oph (Kamata et al. 1997; Grosso 2001), Orion (Ozawa et al. 1999), Taurus (Skinner et al. 1997, see also Carkner et al. 1998), NGC 1333 (Preibisch 1997b; Preibisch et al. 1998), and Serpens (Preibisch 1998). Their luminosities apparently correspond to classical "saturation" $\left(L_{X} / L_{\text {bol }} \approx 10^{-3}\right.$, Ozawa et al. 1999), but extreme levels of $L_{X} \approx(6-18) \times 10^{32} \mathrm{erg} \mathrm{s}^{-1}$ have been reported (Preibisch 1998).

Class I protostars have now become accessible in larger numbers thanks to Chandra's and XMM-Newton's hard-band sensitivity (Imanishi et al. 2001a; Preibisch and Zinnecker 2001, 2002; Preibisch 2003b; Getman et al. 2002). Their measured characteristic temperatures are very high, of order 20-40 MK (Tsujimoto et al. 2002; Imanishi et al. 2001a). Some of these values may, however, be biased by strongly absorbed ("missing") softer components in particular in spectra with limited signal-to-noise ratios. It is correspondingly difficult to characterize the $L_{X}$ values in traditional soft X-ray bands for comparison with other stellar sources.

\subsubsection{Flares and magnetic fields}

Direct evidence for magnetic processes and perhaps truly coronal emission came with the detection of flares. Many of these events are exceedingly large, with total soft X-ray energies of up to $\approx 10^{37} \mathrm{erg}$ (Table 4; Koyama et al. 1996; Kamata et al. 1997; Grosso et al. 1997; Ozawa et al. 1999; Imanishi et al. 2001a, and Preibisch 2003a). Imanishi et al. (2003) conducted a systematic comparative analysis of flares from class I-III stars. They interpreted rise times, decay times and temperatures within the framework of MHD models (Shibata and Yokoyama 2002, Sect. 12.9, Fig. 27). The magnetic fields tend to become stronger toward the typically hotter class I flaring sources. Such flares 


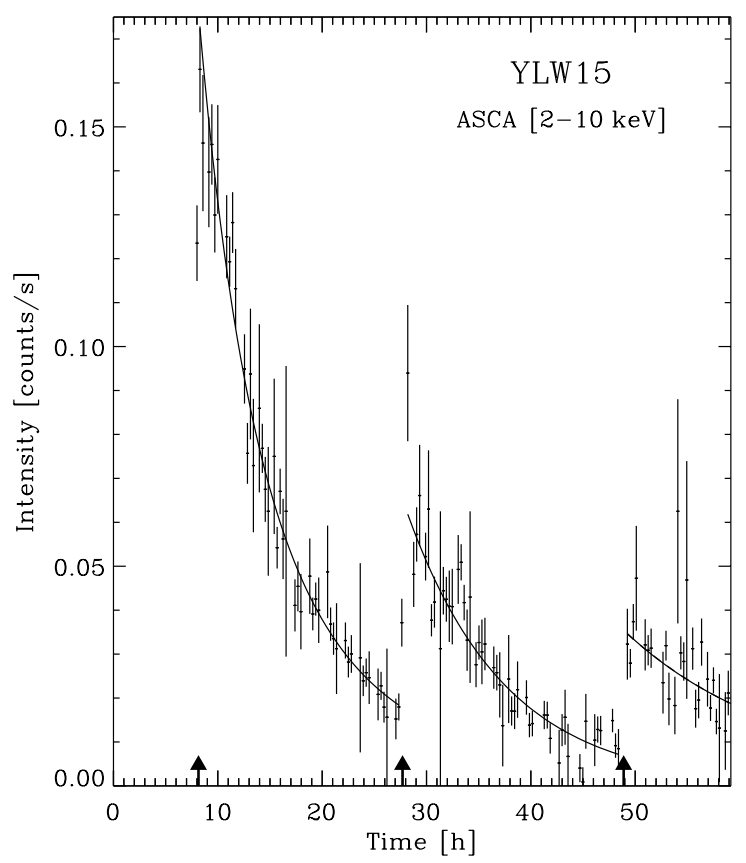

Fig. 46. Triple X-ray flare on the protostar YLW 15, observed with ASCA (figure courtesy of N. Grosso, after Montmerle et al. 2000)

realistically require large volumes, in fact to an extent that star-disk magnetic fields become a possibility for the flaring region (Grosso et al. 1997 for YLW 15 in $\rho$ Oph).

Tsuboi et al. (2000) reported on quasi-periodic flare events in YLW 15 that occurred three times in sequence, separated by about 1 day (Fig. 46). If magnetic fields indeed connect the star with the inner border of the circumstellar disk, then they may periodically ignite flares each time the field lines have become sufficiently stretched due to the difference in rotation rates of the star and the disk (Fig. 47). This scenario was computed by Hayashi et al. (1996); their MHD simulations showed extensive episodic heating and large plasmoids detaching from the star-disk magnetic fields. Montmerle et al. (2000) expanded this view qualitatively to suggest that star-disk magnetic flares should be common in protostars through winding-up star-disk magnetic fields because stellar and inner-disk rotation rates have not synchronized at that age. But because the same fields will eventually brake the star to disk-synchronized rotation in TTS, the large star-disk flares should then cease to occur, and the X-ray activity becomes related exclusively to the stellar corona.

\subsubsection{The stellar environment}

X-ray emission in protostars and strong flaring in particular may have far-reaching consequences for the evolution of the stars themselves, but also for the environment in which planets form. First, X-rays efficiently ionize the molecular environment, which may lead to modifications in the accretion rate, for example via the magnetic Balbus- 


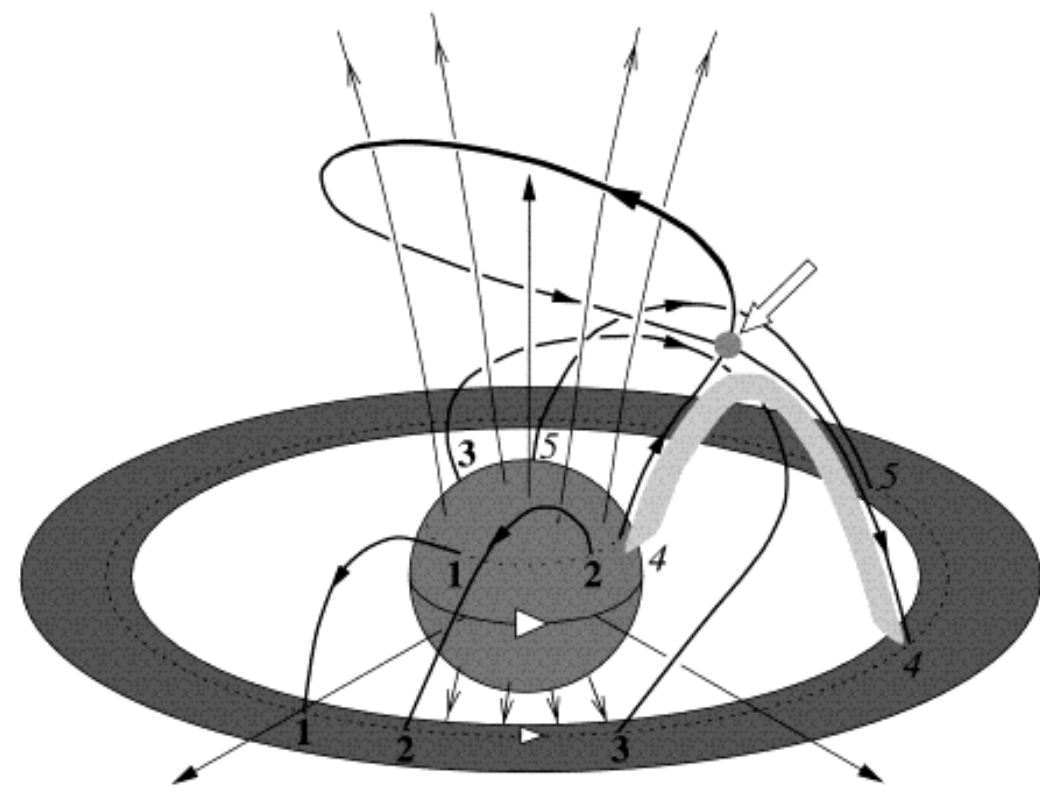

Fig. 47. Sketch illustrating a star-disk magnetic-field model in which field lines wind up and reconnect (line labeled ' 5 ') because the star rotates faster than the inner edge of the disk (figure courtesy of T. Montmerle, after Montmerle et al. 2000)

Hawley instability (see Montmerle et al. 2000 for a discussion). While X-ray flares may thus induce accretion events, the latter will tend to quench strong magnetic activity, in turn decreasing disk ionization. The effect of flare irradiation of disks may indeed have been seen explicitly: Imanishi et al. (2001a) detected, during a giant flare in YLW 16A (in the $\rho$ Oph dark cloud), strong Fe fluorescence line at $6.4 \mathrm{keV}$ that is possibly induced by X-ray irradiation of a circumstellar disk (Fig. 48; see also Koyama et al. 1996). Second, strong, frequent flares and disk ionization may also be of fundamental importance for the generation of jets (Hayashi et al. 1996), spallation reactions in solids in the circumstellar disk, and the formation of planets, a subject beyond the scope of the present review (see, e.g., Feigelson and Montmerle 1999; Feigelson et al. 2002b, and the extensive review of this subject by Glassgold et al. 2000).

\subsection{4. "Class 0" objects}

Knowing that stars are already extremely active at the deeply embedded class I stage, the interest in the magnetic behavior of class 0 objects is obvious, but the strong photoelectric absorption makes detection experiments extremely challenging. Tsuboi et al. (2001) reported a possible detection of a class 0 object in Orion, with properties surprisingly similar to more evolved class I-III objects, such as an X-ray luminosity of $L_{X} \approx 2 \times$ $10^{30} \mathrm{erg} \mathrm{s}^{-1}$, but additional detections and confirmations are badly needed. Specific searches have, so far, given a null result (T. Montmerle 2004, private communication). 


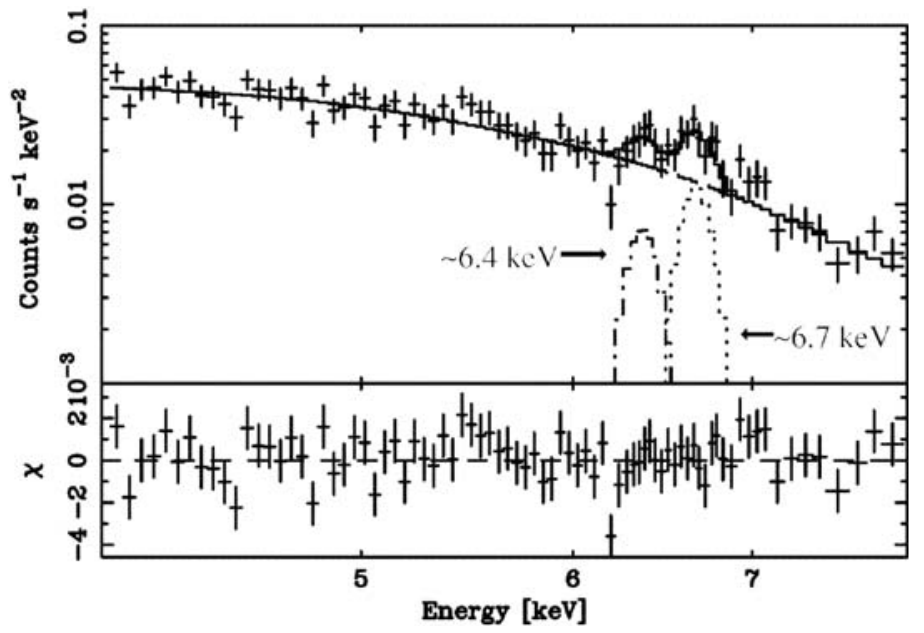

Fig. 48. Chandra CCD spectrum of the protostar YLW 16A observed during a large flare. The spectral line feature at $6.7 \mathrm{keV}$ refers to the Fexxv complex, whereas the line at $6.4 \mathrm{keV}$ is a fluorescent line of cold Fe (figure courtesy of K. Imanishi, after Imanishi et al. 2001a)

\subsection{Young brown dwarfs}

Brown dwarfs (BD) were first detected as faint X-ray sources in star forming regions such as Cha I, Taurus, and $\rho$ Oph (Neuhäuser and Comerón 1998; Neuhäuser et al. 1999; Comerón et al. 2000). They correspond to spectral types beyond M6 at this age. The list of X-ray detections of BDs and candidates is now rapidly growing thanks to sensitive observations with Chandra and XMM-Newton. Considerable numbers have been reported from the Orion Nebula Cluster (Garmire et al. 2000; Feigelson et al. 2002a; Flaccomio et al. 2003b), $\rho$ Oph (Neuhäuser et al. 1999; Imanishi et al. 2001b), IC 348 (Preibisch and Zinnecker 2001, 2002), Taurus (Mokler and Stelzer 2002; Neuhäuser et al. 1999 using ROSAT), and the $\sigma$ Ori cluster (Mokler and Stelzer 2002). The latter authors and Tsuboi et al. (2003) provided a summary of all measurements and put the Xray properties in a wider context. Although alternative origins of the $\mathrm{X}$-rays are possible such as primordial magnetic fields or star-disk fields (see Comerón et al. 2000), all Xray properties suggest thermal emission from a solar-like corona. Specifically, $L_{X}$ is typically found to follow the saturation law $\left(L_{X} / L_{\mathrm{bol}} \approx 10^{-4}-10^{-3}\right)$ of more massive stars, implying a general decrease toward later spectral types, without any evident break. In absolute terms, $L_{X}$ reaches up to a few times $10^{28} \mathrm{erg} \mathrm{s}^{-1}$. Young BDs reveal coronal temperatures typically exceeding $1 \mathrm{keV}$, similar to TTS. A number of these objects have also been found to flare (Imanishi et al. 2001b; Feigelson et al. 2002a). It is only beyond $10^{7} \mathrm{yr}$ that the X-ray emission from BDs decays (see also Sect. 4.3) - again seemingly similar to main-sequence stars (Fig. 49).

No thorough study of relations between accretion disk signatures and X-rays is available at this time. For $\rho$ Oph, Imanishi et al. (2001b) found no relation between $L_{X}$ and $K$ band luminosity excess. However, Tsuboi et al. (2003) suggested that the lack of X-ray detections among stars with very large $\mathrm{H} \alpha$ equivalent widths indicates, as in CTTS, increased accretion at the cost of strong X-ray emission. 

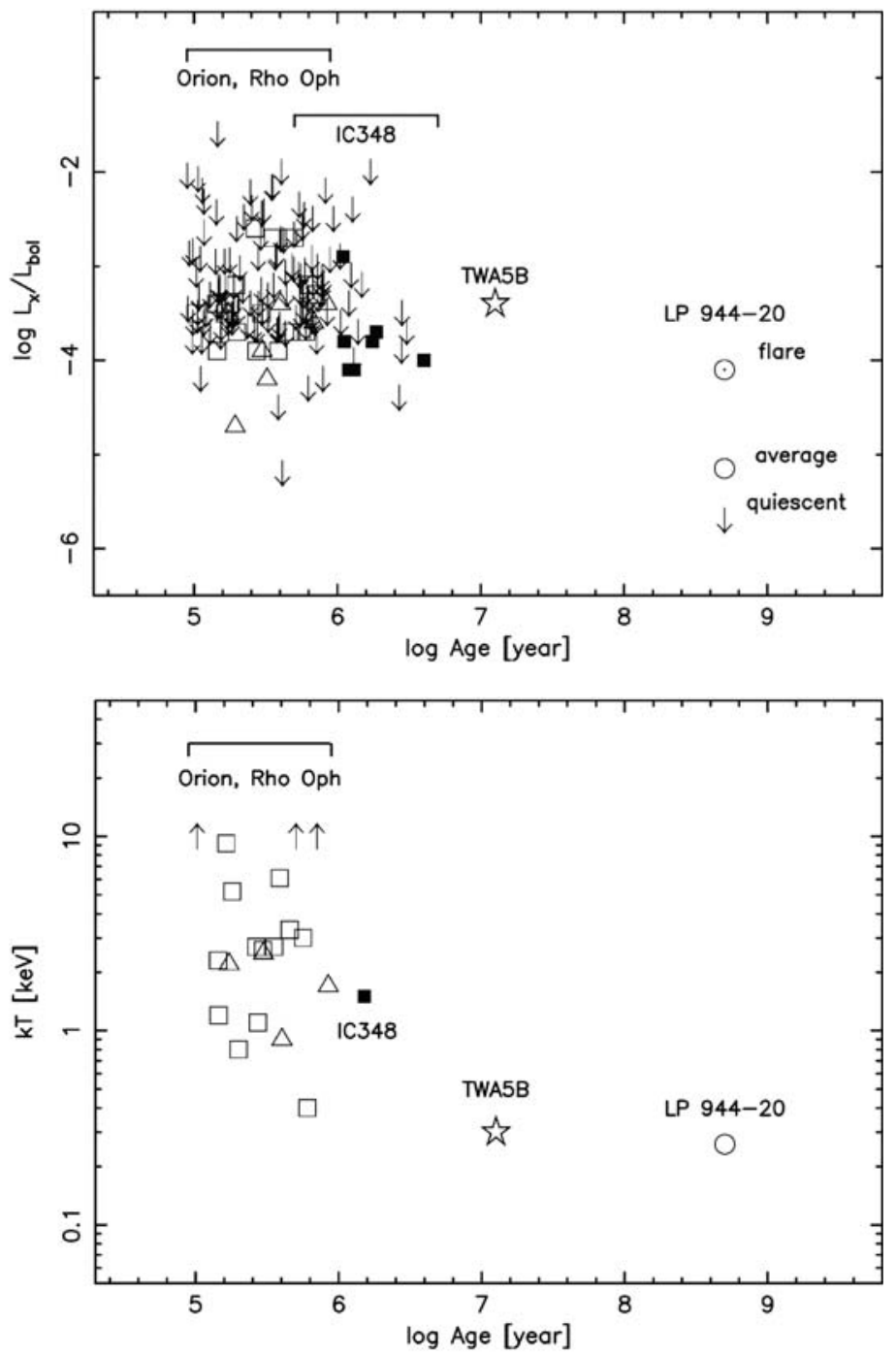

Fig. 49. X-ray characteristics of brown dwarfs as a function of age. Top panel: $\log L_{X} / L_{\text {bol }}$; bottom panel: characteristic electron temperature. Key to the symbols: open squares: Orion Nebula Cluster; triangles: $\rho$ Oph; filled squares: IC 348. All observations were obtained by Chandra (figure courtesy of Y. Tsuboi, after Tsuboi et al. 2003)

The similar behavior of young BDs and TTS is perhaps not entirely surprising, whatever the internal dynamo mechanism is. At this young age, both classes of stars are descending the Hayashi track, are fully convective, have surface temperatures like cool main-sequence dwarfs, and derive most of their energy from gravitational contraction, while they lack a significant central nuclear energy source. Young BDs are not yet aware of their fate to fail as stars. 


\section{Young populations in the solar neighborhood}

Stellar coronae have also proven practical in investigations of (nearby) galactic structure. Large statistical samples of stellar X-ray sources are interesting to compare with galactic population models, for example to uncover "excess populations" of active stars or to assess the stellar contribution to the apparently diffuse galactic soft X-ray background. Sophisticated models take into account the statistical galactic distribution of stellar populations (in terms of scale heights, space densities, etc.) as a function of their color, evolutionary status, and binarity, together with effects of extinction (Favata et al. 1992; Guillout et al. 1996). I will only touch upon this field and mention some of the recent key results.

When observations of large samples were compared with predictions from galactic population models, an excess of nearby "yellow" (F, G, K) stars was found (Favata et al. 1988). Later investigations interpreted them as predominantly young $(<1 \mathrm{Gyr})$, nearZAMS main-sequence stars (Sciortino et al. 1995). RS CVn binaries may contribute as well, but their number is not sufficient to account for the observed excess (Favata et al. 1995a,b). A young population is also conspicuous in the ROSAT All-Sky Survey, providing evidence for a considerable number of such stars in the solar neighborhood. Population studies have further been used to derive constraints on the stellar birthrate. Micela et al. (1993) found that the observed source counts are compatible with a nearly constant galactic stellar birthrate and exclude a rapid decline during the first few Gyr of the galaxy's life.

Guillout et al. (1998a) and Guillout et al. (1998b) undertook a large correlation study between the ROSAT All-Sky Survey and the Tycho Catalog. They found rather compelling evidence for a concentration of luminous and therefore probably young stars along a great circle on the sky that corresponds to the Gould Belt. This local galactic, disk-like structure in the solar vicinity spans over a few hundred parsec and comprises a number of the prominent nearby star-forming regions. The detected population of X-ray stars appears to correspond to the low-mass population of this structure.

\section{Long-term variability and stellar magnetic cycles}

Records of sunspot numbers back over several hundred years show a near-cyclic modulation that has turned out to be a central challenge for dynamo theories. The magnetic activity period between two successive spot maxima of approximately 11 years expresses itself most beautifully in soft X-rays, more so than in any longer wavelength range (see Fig. 1). The X-ray luminosity variation between maximum and minimum has been quoted variably as ranging between a factor of about ten (Aschwanden 1994; Acton 1996) as measured by Yohkoh and GOES, to factors of 20-200 (Kreplin 1970) as measured with SOLRAD, although the spectral bandpasses clearly matter as well for a closer comparison. Converted to the ROSAT bandpass, Hempelmann et al. (1996) estimated variations by a factor of 10, whereas Ayres et al. (1996) found a somewhat more modest factor of 4 as extrapolated from far-UV data.

The Mount Wilson HK project (Baliunas et al. 1995) has collected a continuous data stream of the chromospheric H\&K line flux diagnostic for many stars over several decades. This stupendous observing project has now clearly demonstrated that many 
stars show magnetic activity cycles somewhat similar to the Sun's. A subset of stars appear to lack such cycles, however, and very active stars tend to exhibit an irregular rather than a cyclic mode of variability (Hempelmann et al. 1996).

The sensitive response of the coronal luminosity to changes in the surface magnetic field should make us believe that cycles are easily seen in X-ray active stars. Surprisingly, evidence is still tentative at the time of writing. Magnetic X-ray cycles have eluded detection because no appropriate program has been carried out for sufficiently long periods; however, there may also be interesting physical reasons for a lack of detections. Two strategies toward detecting X-ray cycles have been followed, the first observing young open clusters or field stars with various satellites to obtain statistical information on long-term variability, and the second approach concentrating on dedicated long-term "monitoring" of select field stars.

\subsection{Clusters and field star samples}

Many open clusters or field star samples were observed by Einstein, EXOSAT, and ROSAT, and they obtain new visits again with XMM-Newton and Chandra. One of the principal results of comparative studies over time scales of 10 years has in fact been a suspicious absence of strong long-term variability. Most of these observing programs reported variations of no more than a factor of two for the large majority of stars. Such results apply to samples of active field dMe stars (Pallavicini et al. 1990a), dM dwarfs (Marino et al. 2000), dF-dK stars (Marino et al. 2002), volume-limited samples of nearby stars (Schmitt et al. 1995; Fleming et al. 1995), old disk and halo stars (Micela et al. 1997a), RS CVn binaries (Dempsey et al. 1993a), T Tau stars (Sciortino et al. 1998; Gagné and Caillault 1994; Gagné et al. 1995b; Grosso et al. 2000) and, most notably, open clusters such as the Pleiades (Gagné et al. 1995a; Micela et al. 1996; Marino et al. 2003b) and the Hyades (Stern et al. 1994, 1995b). Much of the observed variability is statistically consistent with shorter-term flare-like fluctuations or variability due to slow changes in active regions (Ambruster et al. 1987). In some cases, there is a modest excess of long-term over short-term variations (e.g., Gagné et al. 1995a for the Pleiades, Marino et al. 2002 for F-K field stars) but the evidence remains marginal.

These investigations were put on a solid statistical footing by comparing short-term with long-term variability of a large sample of active binaries (Kashyap and Drake 1999). Again, most of the variability was identified to occur on time scales $\lesssim 2$ yr although there is marginal evidence for excess variability on longer terms (see also Marino et al. 2000). The latter could then be attributed to cyclic variability although the ratio of maximum to minimum luminosity would be bounded by a factor of 4 , much less than in the case of the Sun. Overall, such results may be taken as evidence for the operation of a distributed dynamo producing relatively unmodulated small-scale magnetic fields (Kashyap and Drake 1999; J. Drake et al. 1996). If this is the case, then it would support a model in which not only the latest M dwarfs, but also earlier-type magnetically active stars are prone to a turbulent dynamo (Weiss 1993).

The long-term variability in stellar coronae was further quantified with a large ROSAT sample of observations by Micela and Marino (2003). They modeled the $\approx 10 \mathrm{yr} X$-ray light curve collected from Yohkoh full-disk observations (Fig. 1) by transforming it to the ROSAT bandpass. Samples of stellar snapshot observations can then be compared 
with the distribution of the solar amplitude variations for a given time separation. In the solar case, statistical variation within a factor of two is due to short-term variability, while another factor of three to four is contributed by the solar cycle. Turning to the stellar sample, the authors concluded that there is some likelihood for inactive stars to have magnetic X-ray cycles somewhat similar to the Sun's, whereas the most active stars tend to be less variable on long time scales.

Hempelmann et al. (1996) attacked the problem by measuring surface flux $F_{X}$ for a number of stars with known $\mathrm{Ca} H \& \mathrm{~K}$ activity cycles. They then compared the excess flux with predictions from a rotation-activity relation based on the known $\mathrm{Ca} H \& \mathrm{~K}$ cycle phase, finding tentative indications that the $\mathrm{X}$-ray flux varies in concert with $\mathrm{Ca}$ along the cycle, but again, the significance is low.

\subsection{Case studies}

Obviously, dedicated long-term programs are in order to obtain more sensitive results on cyclic behavior. First tentative evidence from such a program was reported by Dorren et al. (1995). The star under scrutiny, EK Draconis, is a Pleiades-age solar analog near Xray saturation and is therefore not the type of star for which we expect regular cycles. The evidence for the latter is, however, clear and compelling, with a cycle period of about ten years in optical photometry (measuring the spot coverage) and partly in Ca II and Mg II lines (Dorren and Guinan 1994; Dorren et al. 1995). This cycle has now been followed over two full periods (Güdel et al. 2003c). The star has obtained regular coverage with Xray satellites for over ten years. Initial results were presented in Dorren et al. (1995). The attempt to detect an X-ray cycle is illustrated in Fig. 50. While there is a suggestive anticorrelation between X-ray flux and photospheric brightness where the star is brightest at its activity minimum, the evidence must be considered as tentative. The snapshots were partly obtained with different detectors, and the variation is rather modest, i.e., within a factor of two, illustrating the challenges of such observing programs.

A more recent report by Hempelmann et al. (2003) indicated a correlation between X-ray luminosity and the Ca H\&K S index for the two inactive stars $61 \mathrm{Cyg} \mathrm{A}$ and B. Both show chromospheric modulations on time scales of about 10 years, one being regular and the other irregular, and the X-ray fluxes vary in concert during the $41 / 2$ years of coverage. A gradual X-ray modulation was also seen during a time span 2.5 years in HD 81809, although there seems to be a phase shift by about 1 year with respect to the Ca cycle (Favata et al. 2004). The evidence in these three examples, while promising, should again be regarded as preliminary given that only 30-50\% of the cycle has been covered, and the variations in $61 \mathrm{Cyg}$ are - so far - again within a factor of only $\approx 2$.

\section{Outlook}

Stellar coronal X-ray astronomy enters its fourth decade now, with no lack of wonderful instrumentation available to pursue its goals. Its - so far - short history started with feeble detections of exotic sources in the pioneering seventies; the eighties consolidated our view of X-ray coronae and brought them into a perspective of stellar evolution within which we have placed our Sun. The nineties saw rapid progress with increased 


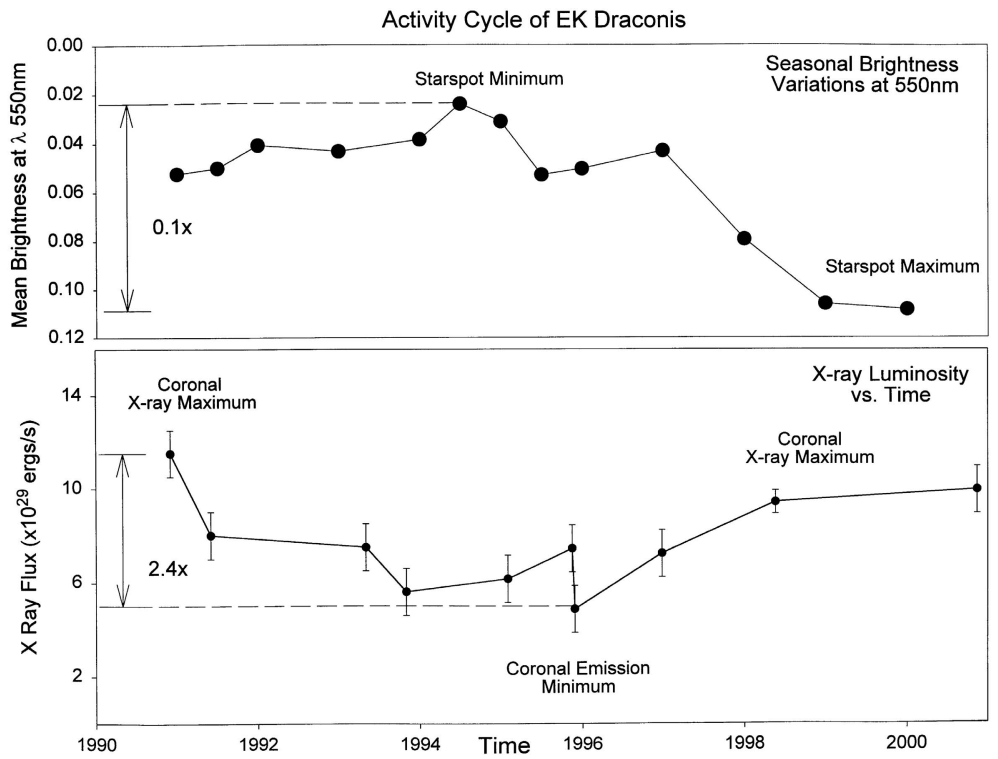

Fig. 50. Illustration of a tentative identification of a magnetic cycle in the corona of EK Dra (G0 V) during one full photospheric magnetic cycle period (about $10 \mathrm{yr}$ ). The upper panel gives brightness offset, maximum brightness referring to smallest starspot coverage. The lower panel shows X-ray luminosity measurements (figure courtesy of E. Guinan)

sensitivities and routine access to spectroscopy, permitting the systematic study of numerous physical processes. And finally, the present decade has brought high-resolution $\mathrm{X}$-ray spectroscopy and high-resolution X-ray imaging, paving the way to deep studies of coronal structure, heating, and evolution. Where should we go from here?

Challenging questions wait to be answered. The following summary of important issues and recommendations represents my current view:

How are coronae heated? The most fundamental question of our subject has started with the very recognition of the presence of hot plasma around the Sun - and it remains unanswered! The subject has not been reviewed in this paper because it is a predominantly solar and plasma-theoretical issue. However, stellar astronomy can shed more light on the problem by making use of what has been called the "solar-stellar connection": through observations of systematic trends over a wide parameter range, while we assume that the basic physical processes remain the same in all stellar systems and the Sun. Stars offer access to plasmas as hot as $100 \mathrm{MK}$. Coronae are observed across a range of stellar gravities, magnetic field strengths, convection zone depths, and rotation periods. The systematic studies of stellar X-ray activity across the HRD have played a key role in the arguments for or against acoustic heating. High-resolution spectroscopy offers new possibilities by studying abundances and the form of DEMs, both being the result of heating processes occurring across the face of a star. The new satellites help us address these issues more systematically.

How are coronae structured? The new, refined observational facilities enhance our diagnostic capabilities that will lead to conclusive insights into the structure of magnet- 
ically confined coronae, magnetic field generation and distribution, and eventually will help us to locate and identify the dynamo mechanism. It is still unclear whether in certain stars $\alpha \omega$ or $\alpha^{2}$ dynamos are in operation. Structure information may provide the necessary boundary conditions as has been discussed in this review, for example in the context of giants. To understand the global structure of a magnetic corona, however, we have to start recognizing structure distribution. Many spectroscopically determined observables such as "density", "temperature", "abundance", or "opacity" are the product of radiation from a plethora of coronal features that are, in fact, distributed in these parameters. Our X-ray spectra provide only a highly degenerate view of a true corona, with many possible realizations for one given observation. The use of painstakingly and accurately determined singular spectroscopic parameters may produce entirely misleading results in our modeling efforts; a value for a density or an element abundance derived from simple line-flux ratios may not reflect the physical conditions of the magnetic features we aim to understand. If we are to succeed in meaningfully describing entire stellar coronae based on spectroscopic data in the future, we must cope with the challenge of modeling statistical distributions of physical parameters across various coronal features. Additionally, new coronal structure reconstruction methods such as X-ray Doppler imaging, to mention at least one, hold promise to gain complementary insight into this problem.

What is the physics behind flares? Although the detailed plasma physical mechanisms of flares can probably only be studied on the Sun, stellar flares offer access to quite extreme situations that may provide important boundary conditions for flare theories. Many characteristics of stellar coronae also suggest contributions of flares. Flare-like processes may be a fundamental mechanism to heat astrophysical plasmas in general. The underlying physical mechanisms certainly deserve more, in-depth observational and theoretical studies.

How does magnetic activity evolve? Although a rough outline of rotation-age-activity relations has been established, many details remain unclear. How is magnetic activity controlled at early stages of evolution? Where are the magnetic fields generated in protostars or their environments, and where and how is magnetic energy released? Do circumstellar accretion disks influence it? Is the accretion process itself important for the generation or suppression of X-ray activity? Do star-disk magnetic fields contribute significantly to X-ray emission? Do they regulate the rotational history of a star that defines the starting conditions for stars settling on the main sequence?

How do magnetic fields and coronal X-rays interact with the stellar environment? It has become clear that X-rays and magnetic fields do not only weakly interact with surrounding planets via a stellar wind and some magnetospheric processes; in young stellar systems, the high levels of X-ray emission may directly alter accretion disk properties by ionization, possibly controlling accretion events and governing, together with associated ultraviolet radiation, the disk and envelope chemistry. Magnetic fields may play a fundamental role in redistributing matter or in making accretion disks unstable. Coronal research turns into "interplanetary research" when dealing with forming young stellar systems.

Many of these aspects are being addressed by present-day observations and theory. But we need to take bolder steps as well, in particular in terms of more sophisticated instrumentation. X-ray spectroscopy is still at an infant stage, essentially separating emission lines but hardly resolving their profiles. With a resolving power exceeding 
$\approx 3000$, rotational broadening, turbulent motions, or bulk plasma motions (e.g., during flares) could become routinely detectable in many stellar sources. "Doppler imaging" then would start really imaging stellar coronae, possibly even for different temperatures, thus producing a 3-D thermal structure model of a stellar corona. The required resolving powers are within reach of current detector technology, for example in the cryogenic domain.

An ultimate goal will be producing spatially resolved images of nearby coronal stars through X-ray interferometric techniques, i.e., the equivalent of what radio astronomy has achieved through very long baseline interferometry. Instead of using a baseline of the Earth's diameter as in conventional radio VLBI, two X-ray telescopes separated by $1.5 \mathrm{~m}$ would - in principle - reach a resolution of 0.3 milliarcsecond at $20 \AA$, sufficient to resolve the diameter of the corona of $\alpha$ Cen into about 30 resolution elements, a resolution that can be compared with early solar X-ray images. First X-ray interferometric laboratory experiments are indeed promising (Cash et al. 2000).

Many of our questions started in the solar system. I consider it a privilege that stellar astronomy is in the position of having an example for close scrutiny nearby. There is hope that further exploiting the solar-stellar connection, i.e., carefully comparing detailed solar studies with investigations of stars, will solve many of the remaining outstanding questions.

Acknowledgements. It is a pleasure to thank the editors of this journal, Profs. L. Woltjer and M. C. E. Huber, for inviting me to write this review, and for their thoughtful comments on the manuscript. I am grateful to many of my colleagues for helpful advice, the contributions of figures, and answers to my persistent questions. Kaspar Arzner, Marc Audard, and Kevin Briggs critically commented on the text. I extend special thanks to them and to Stephen Skinner and Ton Raassen for discussions on our subject over the past many years. I owe much to the late Rolf Mewe who was a prolific source of inspiration and motivation in all aspects of coronal physics and spectroscopy. Discussions with Roberto Pallavicini, Antonio Maggio, and Giovanni Peres helped clarify issues on coronal loops. I acknowledge permission from several colleagues to use their previously published figures or data, in particular: Drs. Marc Audard, Tom Ayres, Nancy Brickhouse, Kevin Briggs, Jeremy Drake, Fabio Favata, Eric Feigelson, Thomas Fleming, Elena Franciosini, Mark Giampapa, Nicolas Grosso, Edward Guinan, Kensuke Imanishi, Moira Jardine, Antonietta Marino, Giusi Micela, Thierry Montmerle, Jan-Uwe Ness, Rachel Osten, Ton Raassen, Sofia Randich, Karel Schrijver, Kazunari Shibata, Marek Siarkowski, Stephen Skinner, Beate Stelzer, Alessandra Telleschi, Yohko Tsuboi, and Takaaki Yokoyama. The figures are reproduced with the permission of the publishers. I am particularly indebted to M. Audard, T. Ayres, N. Brickhouse, G. Micela, J.-U. Ness, R. Osten, M. Siarkowski, K. Shibata, A. Telleschi, and T. Yokoyama for their generous help by preparing entirely new figures for the present article, and Doris Lang for her editorial support. Several previously unprocessed and unpublished data sets or related results are shown in this paper, namely from XMM-Newton, Chandra, GOES, Yohkoh, and TRACE. XMM-Newton is an ESA science mission with instruments and contributions directly funded by ESA Member States and the USA (NASA). The GOES soft X-ray data shown in Fig. 32 are from the Space Environment Center, Boulder, CO, of the US National Oceanic and Atmospheric Administration (NOAA). The solar X-ray images in Fig. 1 were taken by the Yohkoh mission of ISAS, Japan, which is operated by international collaboration of Japanese, US, and UK scientists under the support of ISAS, NASA, and SERC, respectively. Figs. 14 and 24 were obtained by the Transition Region and Coronal Explorer, TRACE, which is a mission of the Stanford-Lockheed Institute for Space Research (a joint program of the Lockheed-Martin Advanced Technology 
Center's Solar and Astrophysics Laboratory and Stanford's Solar Observatories Group), and part of the NASA Small Explorer program. Use was made of the Lausanne open cluster database at http://obswww.unige.ch/webda/, and of the SIMBAD database, operated at CDS, Strasbourg, France. General stellar X-ray astronomy research at PSI has been supported by the Swiss National Science Foundation under projects 2100-049343.96, 20-58827.99, and 20-66875.01.

\section{References}

L. Acton: in 9th Cambridge Workshop on Cool Stars, Stellar Systens, and the Sun, eds. R. Pallavicini, A.K. Dupree, ASP, San Francsico, p. 45 (1996)

P.C.Agrawal, T.H. Markert, G.R. Riegler: MNRAS 213, 761 (1985)

P.C. Agrawal, A.R. Rao, G.R. Riegler: MNRAS 219, 777 (1986b)

P.C. Agrawal, A.R. Rao, B.V. Sreekantan: MNRAS 219, 225 (1986a)

P.C. Agrawal, G.R. Riegler, N.E. White: MNRAS 196, 73P (1981)

P.C. Agrawal, J. Vaidya: MNRAS 235, 239 (1988)

J.M. Alcalá, C. Chavarría-K., L. Terranegra: A\&A 330, 1017 (1998)

J.M. Alcalá, E. Covino, G. Torres, M.F. Sterzik, M.J. Pfeiffer, R. Neuhäuser: A\&A 353, 186 (2000)

J.M. Alcalá, J. Krautter, E. Covino, R. Neuhäuser, J.H.M.M. Schmitt, R. Wichmann: $A \& A$ 319, 184 (1997)

J.M. Alcalá, J. Krautter, J.H.M.M. Schmitt, E. Covino, R. Wichmann, R. Mundt: A\&AS 114, 109 (1995)

C.W. Ambruster, S. Sciortino, L. Golub: ApJS 65, 273 (1987)

C. Ambruster, W.A. Snyder, K.S. Wood: ApJ 284, 270 (1984)

E. Anders, N. Grevesse: Geochim. Cosmochim. Acta 53, 197 (1989)

S.K. Antiochos: ApJ 241, 385 (1980)

S.K. Antiochos, B.M. Haisch, R.A. Stern: ApJ 307, L55 (1986)

S.K. Antiochos, G. Noci: ApJ 301, 440 (1986)

S.K. Antiochos, P.A. Sturrock: Solar Phys. 49, (1976) 359

S.K. Antiochos, P.A. Sturrock: ApJ 220, 1137 (1978)

A. Antunes, F. Nagase, N.E. White: ApJ 436, L83 (1994)

C. Argiroffi, A. Maggio, G. Peres: A\&A 404, 1033 (2003)

K. Arzner, M. Güdel: ApJ 602, 363 (2004)

M. Aschwanden: Sol. Phys. 152, 53 (1994)

M.J. Aschwanden, R.W. Nightingale, D. Alexander: ApJ 541, 1059 (2000a)

M. Aschwanden, C.J Schrijver: ApJS 142, 269 (2002)

M.J. Aschwanden, T.D. Tarbell, R.W. Nightingale, C.J. Schrijver, A. Title, C.C. Kankelborg, P.C.H. Martens, H.P. Warren: ApJ 535, 1047 (2000b)

M. Audard, E. Behar, M. Güdel, A.J.J. Raassen, D. Porquet, R. Mewe, C.R. Foley, G.E. Bromage: $A \& A 365$, L329 (2001)

M. Audard, M. Güdel, J.J. Drake, V. Kashyap: ApJ 541, 396 (2000)

M. Audard, M. Güdel, E.F. Guinan: ApJ 513, L53 (1999)

M. Audard, M. Güdel, R. Mewe: A\&A 365, L318 (2001a)

M. Audard, M. Güdel, S.L. Skinner: ApJ 589, 983 (2003b)

M. Audard, M. Güdel, A. Sres, A.J.J. Raassen, R. Mewe: A\&A 398, 1137 (2003a)

M. Audard, A. Telleschi, M. Güdel, S.L. Skinner, R. Pallavicini, U. Mitra-Kraev: ApJ, in press (2004)

T.R. Ayres: ApJ submitted (2004)

T.R. Ayres, A. Brown, G.M. Harper: ApJ 598, 610 (2003a)

T.R. Ayres, A. Brown, G.M. Harper, P.D. Bennett, J.L. Linsky, K.G. Carpenter, R.D. Robinson: ApJ 491, 876 (1997) 
T.R. Ayres, A. Brown, G.M. Harper, R.A. Osten, J.L. Linsky, B.E. Wood, S. Redfield: ApJ 583, $963(2003 b)$

T.R. Ayres, A. Brown, R.A. Osten, D.P. Huenemoerder, J.J. Drake, N.S. Brickhouse, J.L. Linsky: ApJ 549, 554 (2001b)

T.R. Ayres, T.A. Fleming, J.H.M.M. Schmitt: ApJ 376, L45 (1991)

T.R. Ayres, T.A. Fleming, T. Simon, B.M. Haisch, A. Brown, D. Lenz, W. Wamsteker, D. de Martino, C. Gonzales, J. Bonnell, J.M. Mas-Hesse, C. Rosso, J.H.M.M. Schmitt, J. Trümpter, W. Voges, J. Pye, R.C. Dempsey, J.L. Linsky, E.F. Guinan, G.M. Harper, C. Jordan, B.M. Montesinos, I. Pagano, M. Rodonò: ApJS 96, 223 (1995)

T.R. Ayres, J.L. Linsky: ApJ 241, 279 (1980)

T.R. Ayres, J.L. Linsky, G.S. Vaiana, L. Golub, R. Rosner: ApJ 250, 293 (1981a)

T.R. Ayres, N.C. Marstad, J.L. Linsky: ApJ 247, 545 (1981b)

T.R. Ayres, R.A. Osten, A. Brown: ApJ 526, 445 (1999)

T.R. Ayres, R.A. Osten, A. Brown: ApJ 562, L83 (2001a)

T.R. Ayres, T. Simon, J.L. Linsky: ApJ 263, 791 (1982)

T.R. Ayres, T. Simon, J.R. Stauffer, R.A. Stern, J.P. Pye, A. Brown: ApJ 473, 279 (1996)

T.R. Ayres, T. Simon, R.A. Stern, S.A. Drake, B.E. Wood, A. Brown: ApJ 496, 428 (1998)

J. Babel, T. Montmerle: A\&A 323, 121 (1997)

S. Baliunas, R.A. Donahue, W.H. Soon, J.H. Horne, J. Frazer, L. Woodward-Eklund, M. Bradford, L.M. Rao, O.C. Wilson, Q. Zhang, W. Bennett, J. Briggs, S.M. Carroll, D.K. Duncan, D. Figueroa, H.H. Lanning, T. Misch, J. Mueller, R.W. Noyes, D. Poppe, A.C. Porter, C.R. Robinson, J. Russell, J.C. Shelton, T. Soyumer, A.H. Vaughan, J.H. Whitney: ApJ 438, 269 (1995)

M. Barbera, F. Bocchino, F. Damiani, G. Micela, S. Sciortino, F. Favata, F.R. Harnden Jr.: A\&A 387, 463 (2002)

M. Barbera, G. Micela, S. Sciortino, F.R. Harnden Jr., R. Rosner: ApJ 414, 846 (1993)

D. Barrado Y Navascués, J.R. Stauffer, S. Randich: ApJ 506, 347 (1998)

G. Basri: ApJ 316, 377 (1987)

D.K. Bedford, R.D. Jeffries, E.H. Geyer, O. Vilhu: MNRAS 243, 557 (1990)

E. Behar, J. Cottam, S.M. Kahn: ApJ 548, 966 (2001)

T. Belloni, G. Tagliaferri: $A \& A$ 326, 608 (1997)

T. Belloni, G. Tagliaferri: $A \& A 335,517$ (1998)

T. Belloni, F. Verbunt: $A \& A$ 305, 806 (1996)

T. Belloni, F. Verbunt, R.D. Mathieu: $A \& A$ 339, 431 (1998b)

T. Belloni, F. Verbunt, J.H.M.M. Schmitt: $A \& A$ 269, 175 (1993)

A.O. Benz, J. Conway, M. Güdel: $A \& A$ 331, 596 (1998)

A.O. Benz, M. Güdel: $A \& A$ 285, 621 (1994)

E. Berger: ApJ 572, 503 (2002)

T.W. Berghöfer, J.H.M.M. Schmitt, J.P. Cassinelli: A\&AS 118, 481 (1996)

B.W. Bopp, R.E. Stencel: ApJ 247, L231 (1981)

J. Bouvier: AJ 99, 946 (1990)

J. Bouvier, S. Cabrit, M. Fernández, E.L. Martín, J.M. Matthews: A\&A 272, 176 (1993)

S. Bowyer, J.J. Drake, S. Vennes: ARA\&A 38, 231 (2000)

R.J. Bray, L.E. Cram, C.J. Durrant, R.E. Loughhead: in Plasma Loops in the Solar Corona, Cambridge University Press (1991)

C. Briceño, N. Calvet, S. Kenyon, L. Hartmann: AJ 118, 1354 (1999)

C. Briceño, L.W. Hartmann, J.R. Stauffer, M. Gagné, R.A. Stern, J.-P. Caillault: AJ 113, 740 (1997)

N.S. Brickhouse, A.K. Dupree: ApJ 502, 918 (1998) 
N.S. Brickhouse, A.K. Dupree, R.J. Edgar, D.A. Liedahl, S.A. Drake, N.E. White, K.P. Singh: ApJ 530, 387 (2000)

N.S. Brickhouse, A.K. Dupree, P.R. Young: ApJ 562, L75 (2001)

N.S. Brickhouse, J.C. Raymond, B.W. Smith: ApJS 97, 551 (1995)

K.R. Briggs, J.P. Pye: MNRAS 345, 714 (2003)

K.R. Briggs, J.P. Pye: MNRAS in press (2004)

K.R. Briggs, J.P. Pye, R.D., Jeffries, E.J. Totten: MNRAS 319, 826 (2000)

A.C. Brinkman, E. Behar, M. Güdel, M. Audard, A.J.F. den Boggende, G. Branduardi-Raymont, J. Cottam, C. Erd, J.W. den Herder, F. Jansen, J.S. Kaastra, S.M. Kahn, R. Mewe, F.B.S. Paerels, J.R. Peterson, A.P. Rasmussen, I. Sakelliou, C. de Vries: A\&A 365, L324 (2001)

A.C. Brinkman, C.J.T. Gunsing, J.S. Kaastra, R.L.J. van der Meer, R. Mewe, F. Paerels, A.J.J. Raassen, J.J. van Rooijen, H. Bräuninger, W. Burkert, V. Burwitz, G. Hartner, P. Predehl, J.-U. Ness, J.H.M.M. Schmitt, J.J. Drake, O. Johnson, M. Juda, V. Kashyap, S.S. Murray, D. Pease, P. Ratzlaff, B.J. Wargelin: ApJ 530, L111 (2000)

A. Brown, S.A. Drake, M.E. Van Steenberg, J.L. Linsky: ApJ 373, 614 (1991)

A. Brown, R.A. Osten, S.A. Drake, K.L. Jones, R.A. Stern: in The Hot Universe, IAU Symp 188, eds. K. Koyama, S. Kitamoto, M. Itoh, Kluwer, Dordrecht, p. 215 (1998)

G.V. Brown, P. Beiersdorfer, H. Chen, M.H. Chen, K.J. Reed: ApJ 557, L75 (2001)

G.V. Brown, P. Beiersdorfer, D.A. Liedahl, K. Widmann, S.M. Kahn: ApJ 502, 1015 (1998)

J.C. Brown: Solar Phys. 18, 489 (1971)

T.R. Burnight: Phys. Rev. A 76, 165 (1949)

C.J. Butler: $A \& A$ 272, 507 (1993)

C.J. Butler, M. Rodonò, B.H. Foing, B.M. Haisch: Nature 321, 679 (1986)

P.B. Byrne, D. McKay: A\&A 223, 241 (1989)

J.-P. Caillault, D.J. Helfand: ApJ 289, 279 (1985)

C.R. Canizares, D.P. Huenemoerder, D.S. Davis, D. Dewey, K.A. Flanagan, J. Houck, T.H. Markert, H.L. Marshall, M.L. Schattenburg, N.S. Schulz, M. Wise, J.J. Drake, N.S. Brickhouse: ApJ 539, L41 (2000)

P.J. Cargill: ApJ 422, 381 (1994)

L. Carkner, E.D. Feigelson, K. Koyama, T. Montmerle, I.N. Reid: ApJ 464, 286 (1996)

L. Carkner, J.A. Kozak, E.D. Feigelson: AJ 116, 1933 (1998)

R.W. Carroll, R.G. Cruddace, H. Friedman, E.T. Byram, K. Wood, J. Meekins, D. Yentis, G.H. Share, T.A. Chubb: ApJ 235, L77 (1980)

S. Casanova, T. Montmerle, E.D. Feigelson, P. André: ApJ 439, 752 (1995)

W. Cash, S. Bowyer, P.A. Charles, M. Lampton, G. Garmire, G. Riegler: ApJ 223, L21 (1978)

W. Cash, P. Charles, S. Bowyer, F. Walter, T.R. Ayres, J.L. Linsky: ApJ 231, L137 (1979a)

W. Cash, A. Shipley, S. Osterman, M. Joy: Nature 407, 160 (2000)

W. Cash, T.P. Snow Jr.: ApJ 263, L59 (1982)

W. Cash, T.P. Snow Jr., P. Charles: ApJ 232, L111 (1979b)

R.C. Catura, L.W. Acton, H.M. Johnson: ApJ 196, L47 (1975)

G. Cayrel de Strobel, C. Soubiran, N. Ralite: A\&A 373, 159 (2001)

P. Charles, F. Walter, S. Bowyer: Nature 282, 691 (1979)

C.-C. Cheng, R. Pallavicini: ApJ 381, 234 (1991)

C.S. Choi, T. Dotani: ApJ 492, 761 (1998)

A. Ciaravella, A. Maggio, G. Peres: A\&A 320, 945 (1997)

A. Ciaravella, G. Peres, A. Maggio, S. Serio: A\&A 306, 553 (1996)

A. Collier Cameron: MNRAS 233, 235 (1988)

A. Collier Cameron, D.K. Bedford, S.M. Rucinski, O. Vilhu, N.E. White: MNRAS 231, 131 (1988)

A. Collura, A. Maggio, G. Micela, S. Sciortino, F.R. Harnden Jr., R. Rosner: ApJ 416, 204 (1993)

A. Collura, L. Pasquini, J.H.M.M. Schmitt: A\&A 205, 197 (1988) 
F. Comerón, R. Neuhäuser, A.A. Kaas: $A \& A$ 359, 269 (2000)

E. Covino, J.M. Alcalá, S. Allain, J. Bouvier, L. Terranegra, J. Krautter: A\&A 328, 187 (1997)

S. Covino, M.R. Panzera, G. Tagliaferri, R. Pallavicini: $A \& A$ 371, 973 (2001)

S. Covino, G. Tagliaferri, R. Pallavicini, R. Mewe, E. Poretti: A\&A 355, 681 (2000)

J.D. Craig, J.C. Brown: A\&A 49, 239 (1976)

N.B. Crosby, M.J. Aschwanden, B.R. Dennis: Solar Phys. 143, 275 (1993)

R.G. Cruddace, A.K. Dupree: ApJ 277, 263 (1984)

J.L. Culhane, N.E. White, R.A. Shafer, A.N. Parmar: MNRAS 243, 424 (1990)

S.L. Cully, G. Fisher, M.J. Abbott, O.H.W. Siegmund: ApJ 435, 449 (1994)

S.L. Cully, G.H. Fisher, S.L. Hawley, T. Simon: ApJ 491, 910 (1997)

S.L. Cully, O.H.W. Siegmund, P.W. Vedder, J.V. Vallerga: ApJ 414, L49 (1993)

J. Dachs, W. Hummel: A\&A 312, 818 (1996)

F. Damiani, E. Flaccomio, G. Micela, S. Sciortino, F.R. Harnden Jr., S.S. Murray, S.J. Wolk, R.D. Jeffries: ApJ 588, 1009 (2003)

F. Damiani, G. Micela: ApJ 446, 341 (1995)

F. Damiani, G. Micela, S. Sciortino, F.R. Harnden Jr.: ApJ 446, 331 (1995)

K.J. Daniel, J.L. Linsky, M. Gagné: ApJ 578, 486 (2002)

C. de Jager, J. Heise, S. Avgoloupis, G. Cutispoto, K. Kieboom, R.B. Herr, M. Landini, A.F. Langerwerf, L.N. Mavridis, A.S. Melkonian, R. Molenaar, B.C. Monsignori-Fossi, H.L. Nations, R. Pallavicini, V. Piirola, M. Rodonò, M.A. Seeds, G.H.J. van den Oord, O. Vilhu, C. Waelkens: A\&A 156, 95 (1986)

C. de Jager, J. Heise, A.M. van Genderen, B.H. Foing, I.V. Ilyin, D.S. Kilkenny, S. Avgoloupis, L. Mavridis, G. Cutispoto, M. Rodonò, M.A. Seeds, Yuen K. Ng, W. van Driel, X. Rabattu, A.M. Zodi, J.W.S. Vilas Boas, E. Scarlise, R.E. Schaal, P. Kaufmann, C. Waelkens: A\&A 211, 157 (1989)

R.C. Dempsey, J.L. Linsky, T.A. Fleming, J.H.M.M. Schmitt: ApJS 86, 599 (1993a)

R.C. Dempsey, J.L. Linsky, T.A. Fleming, J.H.M.M. Schmitt: ApJ 478, 358 (1997)

R.C. Dempsey, J.L. Linsky, J.H.M.M. Schmitt, T.A. Fleming: ApJ 413, 333 (1993b)

B.R. Dennis, D.M. Zarro: Solar Phys. 146, 177 (1993)

A.K. Dobson, R.R. Radick: ApJ 344, 907 (1989)

J.-F. Donati: MNRAS 302, 457 (1999)

R. Doron, E. Behar: ApJ 574, 518 (2002)

J.D. Dorren, M. Güdel, E.F. Guinan: ApJ 448, 431 (1995)

J.D. Dorren, E.F. Guinan: ApJ 428, 805 (1994)

G.A. Doschek, U. Feldman, P.B. Landecker, D.L. McKenzie: ApJ 249, 372 (1981)

J.F. Dowdy Jr., R.L. Moore, S.T. Wu: Solar Phys. 99, 79 (1985)

J.G. Doyle, C.J. Butler: Nature 313, 378 (1985)

J.G. Doyle, C.J. Butler, P.B. Byrne, G.H.J. van den Oord: A\&A 193, 229 (1988b)

J.G. Doyle, C.J. Butler, P.J. Callanan, G. Tagliaferri, R. de la Reza, N.E. White, C.A. Torres, G. Quast: $A \& A$ 191, 79 (1988a)

J.G. Doyle, C.J. Butler, B.M. Haisch, M. Rodonò: MNRAS 223, 1P (1986)

J.G. Doyle, B.J. Kellett, C.J. Butler, P.B. Byrne, J.E. Neff, A. Brown, D. Fox, J.L. Linsky, G.E. Bromage, S. Avgoloupis, L.N. Mavridis, J.H. Seiradakis, M. Mathioudakis, H.M. Murphy, J. Krzesinski, G. Pajdosz, V. Dadonas, J. Sperauskas, F. van Wyk, F. Marang, K. Olah, A. Collier Cameron, E. Antonomoulos, P. Rovithis, H. Rovithis-Livaniou: A\&A 96, 351 (1992a)

J.G. Doyle, B.J. Kellett, P.B. Byrne, S. Avgoloupis, L.N. Mavridis, J.H. Seiradakis, G.E. Bromage,

T. Tsuru, K. Makishima, I.M. McHardy: MNRAS 248, 503 (1991)

J.G. Doyle, M. Mathioudakis: A\&A 227, 130 (1990)

J.G. Doyle, G.H.J. van den Oord, B.J. Kellett: $A \& A$ 262, 533 (1992b)

J.J. Drake: ApJ 496, L33 (1998) 
J.J. Drake: ApJ 594, 496 (2003a)

J.J. Drake: Adv. Space Res. 32, 945 (2003b)

J.J. Drake, N.S. Brickhouse, V. Kashyap, J.M. Laming, D.P. Huenemoerder, R. Smith, B.J. Wargelin: ApJ 548, L81 (2001)

J.J. Drake, A. Brown, R.J. Patterer, P.W. Vedder, S. Bowyer, E.F. Guinan: ApJ 421, L43 (1994)

J.J. Drake, V. Kashya: ApJ 547, 428 (2001)

J.J. Drake, J.M. Laming, K.G. Widing: ApJ 443, 393 (1995b)

J.J. Drake, J.M. Laming, K.G. Widing: ApJ 478, 403 (1997)

J.J. Drake, J.M. Laming, K.G. Widing, J.H.M.M. Schmitt, B. Haisch, S. Bowyer: Science 267, 1470 (1995a)

J.J. Drake, G. Peres, S. Orlando, J.M. Laming, A. Maggio: ApJ 545, 1074 (2000)

J.J. Drake, R.A. Stern, G.S. Stringfellow, M. Mathioudakis, J.M. Laming, D.L. Lambert: ApJ 469, 828 (1996)

S.A. Drake, in Cosmic Abundances, eds. S.S. Holt, G. Sonneborn, ASP, San Francisco, p. 215 (1996)

S.A. Drake, D.C. Abbott, T.S. Bastian, J.H. Bieging, E. Churchwell, G. Dulk, J.L. Linsky: ApJS 322, 902 (1987)

S.A. Drake, J.L. Linsky, J.H.M.M. Schmitt, C. Rosso: ApJ 420, 387 (1994b)

S.A. Drake, T. Simon, J.L. Linsky: ApJS 71, 905 (1989)

S.A. Drake, T. Simon, J.L. Linsky: ApJS 82, 311 (1992)

S.A. Drake, K.P. Singh, N.E. White, T. Simon: ApJ 436, L87 (1994a)

A.K. Dupree, N.S. Brickhouse, G.A. Doschek, J.C. Green, J.C. Raymond: ApJ 418, L41 (1993)

B. Edlén: Z. Astrophys. 22, 30 (1942)

M. Endl, K.G. Strassmeier, M. Kürster: A\&A 328, 565 (1997)

F. Favata, M. Barbera, G. Micela, S. Sciortino: $A \& A$ 295, 147 (1995b)

F. Favata, G. Giardino, G. Micela, S. Sciortino, F. Damiani: A\&A 403, 187 (2003)

F. Favata, A. Maggio, G. Peres, S. Sciortino: A\&A 326, 1013 (1997a)

F. Favata, R. Mewe, N.S. Brickhouse, R. Pallavicini, G. Micela, A.K. Dupree: A\&A 324, L37 (1997c)

F. Favata, G. Micela: Space Sci. Rev. 108, 577 (2003b)

F. Favata, G. Micela, S.L. Baliunas, J.H.M.M. Schmitt, M. Güdel, F.R. Harnden Jr., S. Sciortino, R.A. Stern: $A \& A$ 418, L13 (2004)

F. Favata, G. Micela, F. Reale: $A \& A$ 354, 1021 (2000b)

F. Favata, G. Micela, F. Reale: $A \& A$ 375, 485 (2001)

F. Favata, G. Micela, S. Sciortino: $A \& A$ 298, 482 (1995a)

F. Favata, G. Micela, S. Sciortino: $A \& A$ 326, 647 (1997d)

F. Favata, G. Micela, S. Sciortino, G.S. Vaiana: $A \& A$ 256, 86 (1992)

F. Favata, T. Mineo, A.N. Parmar, G. Cusumano: A\&A 324, L41 (1997b)

F. Favata, F. Reale, G. Micela, S. Sciortino, A. Maggio, H. Matsumoto: A\&A 353, 987 (2000a)

F. Favata, R. Rosner, S. Sciortino, G.S. Vaiana: ApJ 324, 1010 (1988)

F. Favata, J.H.M.M. Schmitt: $A \& A$ 350, 900 (1999)

E.D. Feigelson: ApJ 468, 306 (1996)

E.D. Feigelson, P. Broos, J.A. Gaffney III, G. Garmire, L.A. Hillenbrand, S.H. Pravdo, L. Townsley,

Y. Tsuboi: ApJ 574, 258 (2002a)

E.D. Feigelson, S. Casanova, T. Montmerle, J. Guibert: ApJ 416, 623 (1993)

E.D. Feigelson, W.M. DeCampli: ApJ 243, L89 (1981)

E.D. Feigelson, J.A. Gaffney III, G. Garmire, L.A. Hillenbrand, L. Townsley: ApJ 584, 911 (2003)

E.D. Feigelson, G. Garmire, S.H. Pravdo: ApJ 572, 335 (2002b)

E.D. Feigelson, J.M. Jackson, R.D. Mathieu, P.C. Myers, F.M. Walter: AJ 94, 1251 (1987)

E.D. Feigelson, G.A. Kriss: ApJ 248, L35 (1981) 
E.D. Feigelson, G.A. Kriss: ApJ 338, 262 (1989)

E.D. Feigelson, T. Montmerle: ARA\&A 37, 363 (1999)

U. Feldman: Phys. Scripta 46, 202 (1992)

U. Feldman, G.A. Doschek, J.A. Klimchuk: ApJ 474, 511 (1997)

U. Feldman, J.M. Laming, G.A. Doschek: ApJ 451, L79 (1995)

S. Feltzing, J., Holmberg, J.R. Hurley: A\&A 377, 911 (2001)

G.A. Fisher, S.L. Hawley: ApJ 357, 243 (1990)

G.A. Fisher, D.W. Longcope, T.R. Metcalf, A.A. Pevtsov: ApJ 508, 885 (1998)

E. Flaccomio, F. Damiani, G. Micela, S. Sciortino, F.R. Harnden Jr., S.S. Murray, S.J. Wolk: ApJ 582, 382 (2003a)

E. Flaccomio, F. Damiani, G. Micela, S. Sciortino, F.R. Harnden Jr., S.S. Murray, S.J. Wolk: ApJ 582, 398 (2003b)

E. Flaccomio, G. Micela, S. Sciortino: $A \& A$ 402, 277 (2003c)

E. Flaccomio, G. Micela, S. Sciortino: $A \& A$ 397, 611 (2003d)

E. Flaccomio, G. Micela, S. Sciortino, F. Damiani, F. Favata, F.R. Harnden Jr., J. Schachter: $A \& A$ 355,651 (2000)

E. Flaccomio, G. Micela, S. Sciortino, F. Favata, C. Corbally, A. Tomaney: A\&A 345, 521 (1999)

T.A. Fleming, M.S. Giampapa, D. Garza: ApJ 594, 982 (2003)

T.A. Fleming, M.S. Giampapa, J.H.M.M. Schmitt: ApJ 533, 372 (2000)

T.A. Fleming, M.S. Giampapa, J.H.M.M. Schmitt, J.A. Bookbinder: ApJ 410, 387 (1993)

T.A. Fleming, I.M. Gioia, T. Maccacaro: ApJ 340, 1011 (1989)

T.A. Fleming, J. Liebert, I.M. Gioia, T. Maccacaro: ApJ 331, 958 (1988)

T.A. Fleming, J.H.M.M. Schmitt, M.S. Giampapa: ApJ 450, 401 (1995)

T.A. Fleming, G. Tagliaferri: ApJ 472, L101 (1996)

D.C. Fox, J.L. Linsky, A. Vealé, R.C. Dempsey, A. Brown, J.E. Neff, I. Pagano, M. Rodonò, G.E. Bromage, M. Kürster, J.H.M.M. Schmitt: A\&A 284, 91 (1994)

E. Franciosini, R. Pallavicini, T. Bastian, F. Chiuderi-Drago, S. Randich, G. Tagliaferri, M. Massi, J. Neidhöfer: in 11th Cambridge Workshop on Cool Stars, Stellar Systems, and the Sun, eds.

R.J. García López, R. Rebolo, M.R. Zapaterio Osorio, ASP, San Francisco, p. 930 (1999)

E. Franciosini, R. Pallavicini, G. Tagliaferri: A\&A 375, 196 (2001)

E. Franciosini, R. Pallavicini, G. Tagliaferri: $A \& A$ 399, 279 (2003)

E. Franciosini, S. Randich, R. Pallavicini: $A \& A$ 357, 139 (2000)

E. Franciosini, S. Randich, R. Pallavicini: $A \& A$ in press (2004)

M. Freyberg, J.H.M.M. Schmitt: $A \& A$ 296, L21 (1995)

A.H. Gabriel, C. Jordan: MNRAS 145, 241 (1969)

M. Gagné, J.-P. Caillault: ApJ 437, 361 (1994)

M. Gagné, J.-P. Caillault, J.R. Stauffer: ApJ 450, 217 (1995a)

M. Gagné, J.-P. Caillault, J.R. Stauffer: ApJ 445, 280 (1995b)

M. Gagné, J.A. Valenti, J.L. Linsky, G. Tagliaferri, S. Covino, M. Güdel: ApJ 515, 423 (1999)

G.F. Gahm: ApJ 242, L163 (1980)

G. Garmire, E.D. Feigelson, P. Broos, L.A. Hillenbrand, S. Pravdo, L. Townsley, Y. Tsuboi: AJ $120,1426(2000)$

N. Gehrels, E.D. Williams: ApJ 418, L25 (1993)

K.V. Getman, E.D. Feigelson, L. Townsley, J. Bally, C.J. Lada, B. Reipurth: ApJ 575, 354 (2002)

M.S. Giampapa, L. Golub, G. Peres, S. Serio, G.S. Vaiana: ApJ 289, 203 (1985)

M.S. Giampapa, C.F. Prosser, T.A. Fleming: ApJ 501, 624 (1998)

M.S. Giampapa, R. Rosner, V. Kashyap, T.A. Fleming, J.H.M.M. Schmitt, J.A. Bookbinder: ApJ 463, 707 (1996)

G. Giardino, F. Favata, G. Micela, F. Reale: $A \& A$ 669, 679 (2004)

P.A. Gilman: ApJS 53, 243 (1983) 
A.E. Glassgold, E.D. Feigelson, T. Montmerle: in Protostars and Planets IV, eds. V. Mannings, A.P. Boss, S.S. Russell, University of Arizona, Tucson, p. 429 (2000)

L. Golub, F.R. Harnden Jr., C.W. Maxson, R. Rosner, G.S. Vaiana, W. Cash Jr., T.P. Snow Jr.: ApJ 271, 264 (1983)

L. Golub, F.R. Harnden Jr., R. Pallavicini, R. Rosner, G.S. Vaiana: ApJ 253, 242 (1982)

P. Gondoin: $A \& A$ 352, 217 (1999)

P. Gondoin: $A \& A$ 400, 249 (2003a)

P. Gondoin: $A \& A$ 404, 355 (2003b)

P. Gondoin: $A \& A 409,263$ (2003c)

P. Gondoin: $A \& A 415,1113$ (2004a)

P. Gondoin: $A \& A$ 413, 1095 (2004b)

P. Gondoin, C. Erd, D. Lumb: $A \& A$ 383, 919 (2002)

P. Gondoin, A. Mangeney, F. Praderie: $A \& A$ 174, 187 (1987)

E.V. Gotthelf, L. Jalota, K. Mukai, N.E. White: ApJ 436, L91 (1994)

V.G. Graffagnino, D. Wonnacott, S. Schaeidt: MNRAS 275, 129 (1995)

N. Grevesse, A.J. Sauval: A\&A 347, 348 (1999)

N.W. Griffiths: ApJ 518, 873 (1999)

N.W. Griffiths, C. Jordan: ApJ 497, 883 (1998)

N. Grosso: A\&A 370, L22 (2001)

N. Grosso, T. Montmerle, S. Bontemps, P. André, E.D. Feigelson: A\&A 359, 113 (2000)

N. Grosso, T. Montmerle, E.D. Feigelson, P. André, S. Casanova, J. Gregorio-Hetem: Nature 387, 56 (1997)

W. Grotrian: Naturw 27, 214 (1939)

M. Güdel: ApJ 480, L121 (1997)

M. Güdel: ARA\&A 40, 217 (2002)

M. Güdel, K. Arzner, M. Audard, R. Mewe: A\&A 403, 155 (2003b)

M. Güdel, M. Audard, K. Briggs, F. Haberl, H. Magee, A. Maggio, R. Mewe, R. Pallavicini, J. Pye: A\&A 365, L336 (2001b)

M. Güdel, M. Audard, V.L. Kashyap, J.J. Drake, E.F. Guinan: ApJ 582, 423 (2003a)

M. Güdel, M. Audard, H. Magee, E. Franciosini, N. Grosso, F.A. Cordova, R. Pallavicini, R. Mewe: A\&A 365, L344 (2001a)

M. Güdel, M. Audard, F. Reale, S.L. Skinner, J.L. Linsky: A\&A 416, 713 (2004)

M. Güdel, M. Audard, S.L. Skinner, M.I. Horvath: ApJ 580, L73 (2002a)

M. Güdel, M. Audard, K.W. Smith, E. Behar, A.J. Beasley, R. Mewe: ApJ 577, 371 (2002b)

M. Güdel, M. Audard, K.W. Smith, A. Sres, C., Escoda, R. Wehrli, E.F. Guinan, I. Ribas, A.J. Beasley, R. Mewe, A.J.J. Raassen, E. Behar, H. Magee: in 12th Cambridge Workshop on Cool Stars, Stellar Systems, and the Sun, eds. T. Ayres, et al., on-line publication at http://origins.colorado.edu/cs12/, (2003c)

M. Güdel, M. Audard, A. Sres, R. Wehrli, E. Behar, R. Mewe, A.J.J. Raassen, H.R.M. Magee: in Stellar Coronae in the Chandra and XMM-Newton Era, eds. F. Favata, J.J. Drake, ASP, San Francisco, p. 497 (2002c)

M. Güdel, A.O. Benz: ApJ 405, L63 (1993)

M. Güdel, A.O. Benz, J.H.M.M. Schmitt, S.L. Skinner: ApJ 471, 1002 (1996)

M. Güdel, E.F. Guinan, P.B. Etzel, R. Mewe, J.S. Kaastra, S.L. Skinner: in Tenth Cambridge Workshop on Cool Stars, Stellar Systems and the Sun, eds. R. A. Donahue, J.A. Bookbinder, ASP, San Francisco, p. 1247 (1998)

M. Güdel, E.F. Guinan, R. Mewe, J.S. Kaastra, S.L. Skinner: ApJ 479, 416 (1997b)

M. Güdel, E.F. Guinan, S.L. Skinner: ApJ 483, 947 (1997a)

M. Güdel, J.L. Linsky, A. Brown, F. Nagase: ApJ 511, 405 (1999) 
M. Güdel, J.H.M.M. Schmitt: in Röntgenstrahlung from the Universe, eds. H.U. Zimmermann,

J.E. Trümper, H. Yorke, MPE, München, p. 35 (1995)

M. Güdel, J.H.M.M. Schmitt, A.O. Benz, N.M. Elias II: $A \& A$ 301, 201 (1995)

M. Güdel, J.H.M.M. Schmitt, J.A. Bookbinder, T.A. Fleming: ApJ 415, 236 (1993)

P. Guillout, M. Haywood, C. Motch, A.C. Robin: A\&A 316, 89 (1996)

P. Guillout, M.F. Sterzik, J.H.M.M. Schmitt, C. Motch, D. Egret, W. Voges, R. Neuhäuser: $A \& A$ 334, 540 (1998a)

P. Guillout, M.F. Sterzik, J.H.M.M. Schmitt, C. Motch, R. Neuhäuser: A\&A 337, 113 (1998b)

A.G. Gunn, P.A. Brady, V. Migenes, R.E. Spencer, J.G. Doyle: MNRAS 304, 611 (1999)

A.G. Gunn, V. Migenes, J.G. Doyle, R.E. Spencer, M. Mathioudakis: MNRAS 287, 199 (1997)

B.M. Haisch, J.A. Bookbinder, A. Maggio, G.S. Vaiana, J.O. Bennett: ApJ 361, 570 (1990c)

B.M. Haisch, C.J. Butler, J.G. Doyle, M. Rodonò: A\&A 181, 96 (1987)

B.M. Haisch, C.J. Butler, B. Foing, M. Rodonò, M.S. Giampapa: A\&A 232, 387 (1990a)

B. Haisch, J.J. Drake, J.H.M.M. Schmitt: ApJ 421, L39 (1994)

B.M. Haisch, J.L. Linsky, P.L. Bornmann, R.E. Stencel, S.K. Antiochos, L. Golub, G.S. Vaiana: ApJ 267, 280 (1983)

B.M. Haisch, J.L. Linsky, F.R. Harnden Jr., R. Rosner, F.D. Seward, G.S. Vaiana: ApJ 242, L99 (1980)

B.M. Haisch, J.L. Linsky, M. Lampton, F. Paresce, B. Margon, R. Stern: ApJ 213, L119 (1977)

B.M. Haisch, J.L. Linsky, O.B. Slee, B.C. Siegman, I. Nikoloff, M. Candy, D. Harwood, A. Verveer,

P.J. Quinn, I. Wilson, A.A. Page, P. Higson, F.D. Seward: ApJ 245, 1009 (1981)

B. Haisch, J.H.M.M. Schmitt: ApJ 426, 716 (1994)

B.M. Haisch, J.H.M.M. Schmitt, M. Rodonò, D.M. Gibson: A\&A 230, 419 (1990b)

B. Haisch, J.H.M.M. Schmitt, C. Rosso: ApJ 383, L15 (1991b)

B. Haisch, J.H.M.M. Schmitt, C. Rosso: ApJ 388, L61 (1992)

B.M. Haisch, T. Simon: ApJ 263, 252 (1982)

B. Haisch, K.T. Strong, M. Rodonò: ARA\&A 29, 275 (1991a)

K. Hamaguchi, H. Terada, A. Bamba, K. Koyama: ApJ 532, 1111 (2000)

S. Harmer, R.D. Jeffries, E.J. Totten, J.P. Pye: MNRAS 324, 473 (2001)

F.R. Harnden Jr., N.R. Adams, F. Damiani, J.J. Drake, N.R. Evans, F. Favata, E. Flaccomio, P. Freeman, R.D. Jeffries, V. Kashyap, G. Micela, B.M. Patten, N. Pizzolato, J.F. Schachter, S. Sciortino, J. Stauffer, S.J. Wolk, M.V. Zombeck: ApJ 547, L141 (2001)

F.R. Harnden Jr., D. Fabricant, K. Topka, B.P. Flannery, W.H. Tucker, P. Gorenstein: ApJ 214, 418 (1977)

L. Hartmann, A.K. Dupree, J.C. Raymond: ApJ 236, L143 (1980)

A. Hatzes: in Stellar Surface Structure - Poster Proceedings, ed. K.G. Strassmeier, University of Vienna, Vienna, p. 90 (1995)

S.L. Hawley, G.H. Fisher, T. Simon, S.L. Cully, S.E. Deustua, M. Jablonski, C.M. Johns-Krull,

B.R. Pettersen, V. Smith, W.J. Spiesman, J. Valenti: ApJ 453, 464 (1995)

M.R. Hayashi, K. Shibata, R. Matsumoto: ApJ 468, L37 (1996)

J. Heise, A.C. Brinkman, J. Schrijver, R. Mewe, E.H.B.M. Gronenschild, A.J.F. den Boggende, J. Grindlay: ApJ 202, L73 (1975)

A. Hempelmann, J.H.M.M. Schmitt, S.L. Baliunas, R.A. Donahue: A\&A 406, L39 (2003)

A. Hempelmann, J.H.M.M. Schmitt, M. Schultz, G. Rüdiger, K. Stępieǹ: A\&A 294, 515 (1995)

A. Hempelmann, J.H.M.M. Schmitt, K. Stȩpieǹ: A\&A 305, 284 (1996)

J.-C. Hénoux: Adv. Space Res. 15, 23 (1995)

J.S. Hey: Nature 157, 47 (1946)

S.T. Hodgkin, R.F. Jameson, I.A. Steele: MNRAS 274, 869 (1995)

W. Hoff, T. Henning, W. Pfau: A\&A 336, 242 (1998) 
S.S. Holt, N.E. White, R.H. Becker, E.A. Boldt, R.F. Mushotzky, P.J. Serlemitsos, B.W. Smith: ApJ 234, L65 (1979)

H.S. Hudson, L.W. Acton, T. Hirayama, Y. Uchida: PASP 44, L77 (1992)

D.P. Huenemoerder, C.R. Canizares, J.J. Drake, J. Sanz-Forcada: ApJ 595, 1131 (2003)

D.P. Huenemoerder, C.R. Canizares, N.S. Schulz: ApJ 559, 1135 (2001)

M. Hünsch, D. Reimers: A\&A 296, 509 (1995)

M. Hünsch, J.H.M.M. Schmitt, K.-P. Schröder, D. Reimers: A\&A 310, 801 (1996)

M. Hünsch, J.H.M.M. Schmitt, M.F. Sterzik, W. Voges: A\&AS 135, 319 (1999)

M. Hünsch, J.H.M.M. Schmitt, W. Voges: A\&AS 127, 251 (1998a)

M. Hünsch, J.H.M.M. Schmitt, W. Voges: $A \& A S$ 132, 155 (1998b)

M. Hünsch, K.-P. Schröder: A\&A 309, L51 (1996)

M. Hünsch, C. Weidner, J.H.M.M. Schmitt: $A \& A$ 402, 571 (2003)

G.A.J. Hussain, A.A. van Ballegooijen, M. Jardine, A. Collier Cameron: ApJ 575, 1078 (2002)

K. Imanishi, K. Koyama, Y. Tsuboi: ApJ 557, 747 (2001a)

K. Imanishi, M. Nakajima, M. Tsujimoto, K. Koyama, Y. Tsuboi: PASJ 55, 653 (2003)

K. Imanishi, M. Tsujimoto, K. Koyama: ApJ 563, 361 (2001b)

K. Imanishi, M. Tsujimoto, K. Koyama: ApJ 572, 300 (2002)

J. Jakimiec, B. Sylwester, J. Sylwester, S. Serio, G. Peres, F. Reale: A\&A 253, 269 (1992)

D.J. James, M.M. Jardine, R.D. Jeffries, S. Randich, A. Collier Cameron, M. Ferreira: MNRAS 318, 1217 (2000)

D.J. James, R.D. Jeffries: MNRAS 291, 252 (1997)

M. Jardine: $A \& A$ 414, L5 (2004)

M. Jardine, A. Collier Cameron, J.-F. Donati: MNRAS 333, 339 (2002a)

M. Jardine, K. Wood, A. Collier Cameron, J.-F. Donati, D.H. Mackay: MNRAS 336, 1364 (2002b)

M. Jardine, Y.C. Unruh: A\&A 346, 883 (1999)

R.D. Jeffries: MNRAS 295, 825 (1998)

R.D. Jeffries, D.K. Bedford: MNRAS 246, 337 (1990)

R.D. Jeffries, M.R. Thurston, J.P. Pye: MNRAS 287, 350 (1997)

R.D. Jeffries, A.J. Tolley: MNRAS 300, 331 (1998)

E.L.N. Jensen, D.H. Cohen, R. Neuhäuser: AJ 116, 414 (1998)

H.M. Johnson: ApJ 243, 234 (1981)

H.M. Johnson: ApJ 316, 458 (1987)

O. Johnson, J.J. Drake, V. Kashyap, N.S. Brickhouse, A.K. Dupree, P. Freeman, P.R. Young, G.A.

Kriss: ApJ 565, L97 (2002)

C. Jordan: A\&A 86, 355 (1980)

C. Jordan, T.R. Ayres, A. Brown, J.L. Linsky, T. Simon: MNRAS 225, 903 (1987)

C. Jordan, A. Brown, F.M. Walter, J.L. Linsky: MNRAS 218, 465 (1986)

C. Jordan, G.A. Doschek, J.J. Drake, A.B. Galvin, J.C. Raymond: in 10th Cambridge Workshop on Cool Stars, Stellar Systems, and the Sun, eds. R.A. Donahue, J.A. Bookbinder, ASP, San Francisco, p. 91 (1998)

C. Jordan, B. Montesinos: MNRAS 252, 21P (1991)

J.S. Kaastra, R. Mewe, D.A. Liedahl, K.P. Singh, N.E. White, S.A. Drake: A\&A 314, 547 (1996)

S. Kahler, L. Golub, F.R. Harnden Jr., W. Liller, F. Seward, G. Vaiana, B. Lovell, R.J. Davis, R.E. Spencer, D.R. Whitehouse, P.A. Feldman, M.R. Viner, B. Leslie, S.M. Kahn, K.O. Mason, M.M. Davis, C.J. Grannell, R.W. Hobbs, T.J. Schneeberger, S.P. Worden, R.A. Schommer, S.S. Vogt, B.R. Pettersen, G.D. Coleman, J.T. Karpen, M.S. Giampapa, E.K. Hege, V. Pazzani, M. Rodonò, G. Romeo, P.F. Chugainov: ApJ 252, 239 (1982)

S.M. Kahn, J.L. Linsky, K.O. Mason, B.M. Haisch, C.S. Bowyer, N.E. White, S.H. Pravdo: ApJ 234, L107 (1979)

Y. Kamata, K. Koyama, Y. Tsuboi, S. Yamauchi: PASJ 49, 461 (1997) 
V. Kashyap, J.J. Drake: ApJ 524, 988 (1996)

V. Kashyap, J.J. Drake: ApJ 503, 450 (1998)

V. Kashyap, J.J. Drake: ApJ 524, 988 (1999)

V. Kashyap, J.J. Drake, M. Güdel, M. Audard: ApJ 580, 1118 (2002)

V. Kashyap, R. Rosner, F.R. Harnden Jr., A. Maggio, G. Micela, S. Sciortino: ApJ 431, 402 (1994)

J.H. Kastner, D.P. Huenemoerder, N.S. Schulz, C.R. Canizares, D.A. Weintraub: ApJ 567, 434 (2002)

J.H. Kastner, D.P. Huenemoerder, N.S. Schulz, D.A. Weintraub: ApJ 525, 837 (1999)

M.M. Katsova, A.Ya. Boiko, M.A. Livshits: $A \& A$ 321, 549 (1997)

M.M. Katsova, J.J. Drake, M.A. Livshits: ApJ 510, 986 (1999)

R.A. Kopp, G. Poletto: Sol. Phys. 93, 351 (1984)

R.A. Kopp, G. Poletto: ApJ 418, 496 (1993)

T. Kosugi, B.R. Dennis, K. Kai: ApJ 324, 1118 (1988)

K. Koyama, K. Hamaguchi, S. Ueno, N. Kobayashi, E.D. Feigelson: PASJ 48, L87 (1996)

K. Koyama, Y. Maeda, M. Ozaki, S. Ueno, Y. Kamata, Y. Tawara, S. Skinner, S. Yamauchi: PASJ 46, L125 (1994)

J. Krautter, R. Wichmann, J.H.M.M. Schmitt, J.M. Alcalá, R. Neuhäuser, L. Terranegra: $A \& A S$ 123, 329 (1997)

R.W. Kreplin: Ann. Geophys. 26, 567 (1970)

A. Krishnamurthi, C.S. Reynolds, J.L. Linsky, E. Martín, M. Gagné: AJ 121, 337 (2001)

S. Krucker, A.O. Benz: ApJ 501, L213 (1998)

W.H.-M. Ku, G.A. Chanan: ApJ 234, L59 (1979)

W.H.-M. Ku, G. Righini-Cohen, M. Simon: Science 215, 61 (1982)

M.R. Kundu, P.D. Jackson, S.M. White, M. Melozzi: ApJ 312, 822 (1987)

M.R. Kundu, R. Pallavicini, S.M. White, P.D. Jackson: A\&A 195, 159 (1988)

M. Kürster, J.H.M.M. Schmitt: A\&A 311, 211 (1996)

M. Kürster, J.H.M.M. Schmitt, G. Cutispoto, K. Dennerl: A\&A 320, 831 (1997)

J.M. Laming, J.J. Drake: ApJ 516, 324 (1999)

J.M. Laming, J.J. Drake, K.G. Widing: ApJ 443, 416 (1995)

J.M. Laming, J.J. Drake, K.G. Widing: ApJ 462, 948 (1996)

J.M. Laming, I. Kink, E. Takacs, J.V. Porto, J.D. Gillaspy, E.H. Silver, H.W. Schnopper, S.R.

Bandler, N.S. Brickhouse, S.S. Murray, M. Barbera, A.K. Bhatia, G.A. Doschek, N. Madden,

D. Landis, J. Beeman, E.E. Haller: ApJ 545, L161 (2000)

E. Landi, U. Feldman, D.E. Innes, W. Curdt: ApJ 582, 506 (2003)

E. Landi, M. Landini: A\&A 340, 265 (1998)

M. Landini, B.C. Monsignori Fossi, R. Pallavicini, L. Piro: A\&A 157, 217 (1986)

W.A. Lawson, E.D. Feigelson, D.P. Huenemoerder: MNRAS 280, 1071 (1996)

J.R. Lemen, R. Mewe, C.J. Schrijver, A. Fludra: ApJ 341, 474 (1989)

J.K. Lepson, P. Beiersdorfer, G.V. Brown, D.A. Liedahl, S.B. Utter, N.S. Brickhouse, A.K. Dupree,

J.S. Kaastra, R. Mewe, S.M. Kahn: ApJ 578, 648 (2002)

J. Lim, G.J. Nelson, C. Castro, D. Kilkenny, F. van Wyk: ApJ 388, L27 (1992)

J. Lim, S.M. White, S.L. Cully: ApJ 461, 1009 (1996)

R.P. Lin, R.A. Schwartz, S.R. Kane, R.M. Pelling, K. Hurley: ApJ 283, 421 (1984)

J.L. Linsky: Solar Phys. 100, 333 (1985)

J.L. Linsky, B.M. Haisch: ApJ 229, L27 (1979)

J.L. Linsky, B.E. Wood, A. Brown, R. Osten: ApJ 492, 767 (1998)

L.B. Lucy: AJ 79, 745 (1974)

H.R.M. Magee, M. Güdel, M. Audard, R. Mewe: Adv. Space Res. 32, 1149 (2003)

A. Maggio, F. Favata, H. Negoro, N.S. Brickhouse, A.K. Dupree: A\&A 352, 248 (1999)

A. Maggio, F. Favata, G. Peres, S. Sciortino: A\&A 330, 139 (1998) 
A. Maggio, R. Pallavicini, F. Reale, G. Tagliaferri: $A \& A$ 356, 627 (2000)

A. Maggio, G. Peres: $A \& A$ 306, 563 (1996)

A. Maggio, G. Peres: A\&A 325, 237 (1997)

A. Maggio, S. Sciortino, F.R. Harnden Jr.: ApJ 432, 701 (1994)

A. Maggio, S. Sciortino, G.S. Vaiana, P. Majer, J.A. Bookbinder, L. Golub, F.R. Harnden Jr., R. Rosner: ApJ 315, 687 (1987)

A. Maggio, G.S. Vaiana, B.M. Haisch, R.A. Stern, J. Bookbinder, F.R. Harnden Jr., R. Rosner: ApJ 348, 253 (1990)

P. Majer, J.H.M.M. Schmitt, L. Golub, F.R. Harnden Jr., R. Rosner: ApJ 300, 360 (1986)

E.E. Mamajek, W.A. Lawson, E.D. Feigelson: ApJ 516, L77 (1999)

E.E. Mamajek, W.A. Lawson, E.D. Feigelson: ApJ 544, 356 (2000)

A. Mangeney, F. Praderie: $A \& A$ 130, 143 (1984)

S.P. Maran, R.D. Robinson, S.N. Shore, J.W. Brosius, K.G. Carpenter, B.E. Woodgate, J.L. Linsky, A. Brown, P.B. Byrne, M.R. Kundu, S. White, J.C. Brandt, R.A. Shine, F.M. Walter: ApJ 421, 800 (1994)

A. Marino, G. Micela, G. Peres: $A \& A$ 353, 177 (2000)

A. Marino, G. Micela, G. Peres, G., S. Sciortino: $A \& A$ 383, 210 (2002)

A. Marino, G. Micela, G. Peres, S. Sciortino: $A \& A$ 407, L63 (2003a)

A. Marino, G. Micela, G. Peres, S. Sciortino: $A \& A$ 406, 629 (2003b)

J.T. Mariska, A.G. Emslie, P. Li: ApJ 341, 1067 (1989)

P.C.H. Martens, N.P.M. Kuin: $A \& A$ 112, 366 (1982)

E.L. Martín, A. Magazzù: $A \& A$ 342, 173 (1999)

M. Mathioudakis, J.G. Doyle: $A \& A$ 224, 179 (1989)

M. Mathioudakis, J.G. Doyle: $A \& A$ 240, 357 (1990)

M. Mathioudakis, D.J. Mullan: A\&A 342, 524 (1999)

G.E. McCluskey Jr., Y. Kondo: PASP 96, 817 (1984)

P.A. McGale, J.P. Pye, C.R. Barber, C.G. Page: MNRAS 275, 1232 (1995)

P.A. McGale, J.P. Pye, S.T. Hodgkin: MNRAS 280, 627 (1996)

D.L. McKenzie, R.M. Broussard, P.N. Landecker, H.R. Rugge, R.M. Young, G.A. Doschek, U. Feldman: ApJ 238, L43 (1980)

R. Mewe: $A \& A R$ 3, 127 (1991)

R. Mewe, S.A. Drake, J.S. Kaastra, C.J. Schrijver, J.J. Drake, M. Güdel, J.H.M.M. Schmitt, K.P. Singh, N.E. White: A\&A 339, 545 (1998a)

R. Mewe, E.H.B.M. Gronenschild, G.H.J. van den Oord: $A \& A S$ 62, 197 (1985)

R. Mewe, E.H.B.M. Gronenschild, N.J. Westergaard, J. Heise, F. Seward, T. Chlebowski, N.P.M. Kuin, A.C. Brinkman, J.H. Dijkstra, H.W. Schnopper: ApJ 260, 233 (1982)

R. Mewe, M. Güdel, F. Favata, J.S. Kaastra: A\&A 340, 216 (1998b)

R. Mewe, J. Heise, E.H.B.M. Gronenschild, A.C. Brinkman, J. Schrijver, A.J.F. den Boggende: ApJ 202, L67 (1975)

R. Mewe, J.S. Kaastra, C.J. Schrijver, G.H.J. van den Oord, F.J.M. Alkemade: A\&A 296, 477 (1995)

R. Mewe, J.S. Kaastra, G.H.J. van den Oord, J. Vink, Y. Tawara: A\&A 320, 147 (1997)

R. Mewe, J.S. Kaastra, S.M. White, R. Pallavicini: A\&A 315, 170 (1996)

R. Mewe, A.J.J. Raassen, J.J. Drake, J.S. Kaastra, R.L.J. van der Meer, D. Porquet: A\&A 368, 888 (2001)

R. Mewe, C.J. Schrijver: $A \& A$ 169, 178 (1986)

J.-P. Meyer: ApJS 57, 151 (1985a)

J.-P. Meyer: ApJS 57, 171 (1985b)

G. Micela, F. Favata, S. Sciortino: A\&A 326, 221 (1997b)

G. Micela, A. Maggio, G.S. Vaiana: ApJ 388, 171 (1992) 
G. Micela, A. Marino: $A \& A$ 404, 637 (2003)

G. Micela, J. Pye, S. Sciortino: A\&A 320, 865 (1997a)

G. Micela, S. Sciortino, F. Favata: ApJ 412, 618 (1993)

G. Micela, S. Sciortino, F. Favata, R. Pallavicini, J. Pye: A\&A 344, 83 (1999b)

G. Micela, S. Sciortino, F.R. Harnden Jr., V. Kashyap, R. Rosner, C.F. Prosser, F. Damiani, J. Stauffer, J.-P. Caillault: A\&A 341, 751 (1999a)

G. Micela, S. Sciortino, R.D. Jeffries, M.R. Thurston, F. Favata: A\&A 357, 909 (2000)

G. Micela, S. Sciortino, V. Kashyap, F.R. Harnden Jr., R. Rosner: ApJS 102, 75 (1996)

G. Micela, S. Sciortino, S. Serio, G.S. Vaiana, J. Bookbinder, L. Golub, F.R. Harnden Jr., R. Rosner: ApJ 292, 171 (1985)

G. Micela, S. Sciortino, G.S. Vaiana, F.R. Harnden Jr., R. Rosner, J.H.M.M. Schmitt: ApJ 348, 557 (1990)

G. Micela, S. Sciortino, G.S. Vaiana, J.H.M.M. Schmitt, R.A. Stern, F.R. Harnden Jr., R. Rosner: ApJ 325, 798 (1988)

U. Mitra-Kraev, L.K. Harra, M. Güdel, M. Audard, G. Branduardi-Raymont, H.R.M. Kay, R. Mewe, A.J.J. Raassen, L. van Driel-Gesztelyi: $A \& A$ submitted (2004)

F. Mokler, B. Stelzer: A\&A 391, 1025 (2002)

B.C. Monsignori Fossi, M. Landini, G. Del Zanna, S. Bowyer: ApJ 466, 427 (1996)

T. Montmerle, N. Grosso, Y. Tsuboi, K. Koyama: ApJ 532, 1097 (2000)

T. Montmerle, L. Koch-Miramond, E. Falgarone, J.E. Grindlay: ApJ 269, 182 (1983)

D.J. Mullan, Q.Q. Cheng: ApJ 420, 392 (1994a)

D.J. Mullan, Q.Q. Cheng: ApJ 435, 435 (1994b)

D.J. Mullan, T.A. Fleming: ApJ 464, 890 (1996)

F. Nagai: Solar Phys. 68, 351 (1980)

J.-U. Ness, N.S. Brickhouse, J.J. Drake, D.P. Huenemoerder: ApJ 598, 1277 (2003b)

J.-U. Ness, M. Güdel, J.H.M.M. Schmitt, M. Audard, A. Telleschi: $A \& A$ in press (2004)

J.-U. Ness, R. Mewe, J.H.M.M. Schmitt, A.J.J. Raassen, D. Porquet, J.S. Kaastra, R.L.J. van der Meer, V. Burwitz, P. Predehl: A\&A 367, 282 (2001)

J.-U. Ness, J.H.M.M. Schmitt, M. Audard, M. Güdel, R. Mewe: A\&A 407, 347 (2003a)

J.-U. Ness, J.H.M.M. Schmitt, V. Burwitz, R. Mewe, P. Predehl: A\&A 387, 1032 (2002b)

J.-U. Ness, J.H.M.M. Schmitt, V. Burwitz, R. Mewe, A.J.J. Raassen, R.L.J. van der Meer, P. Predehl, A.C. Brinkman: A\&A 394, 911 (2002a)

R. Neuhäuser, C. Briceño, F. Comerón, T. Hearty, E.L. Martín, J.H.M.M. Schmitt, B. Stelzer, R. Supper, W. Voges, H. Zinnecker: A\&A 343, 883 (1999)

R. Neuhäuser, F. Comerón: Science 282, 83 (1998)

R. Neuhäuser, T. Preibisch: A\&A 322, L37 (1997)

R. Neuhäuser, M.F. Sterzik, J.H.M.M. Schmitt, R. Wichmann, J. Krautter: A\&A 297, 391 (1995a)

R. Neuhäuser, M.F. Sterzik, J.H.M.M. Schmitt, R. Wichmann, J. Krautter: A\&A 295, L5 (1995c)

R. Neuhäuser, M.F. Sterzik, G. Torres, E.L. Martín: $A \& A$ 299, L13 (1995b)

R. Neuhäuser, G. Torres, M.F. Sterzik, S. Randich: A\&A 325, 647 (1997)

W.M. Neupert: ApJ 153, L59 (1968)

R.W. Noyes, L.W. Hartmann, S.L. Baliunas, D.K. Duncan, A.N. Vaughan: ApJ 279, 763 (1984)

J. Nugent, G. Garmire: ApJ 226, L83 (1978)

S. Orlando, G. Peres, F. Reale: ApJ 528, 524 (2000)

S. Orlando, G. Peres, F. Reale: ApJ 560, 499 (2001)

A. Ortolani, A. Maggio, R. Pallavicini, S. Sciortino, J.J. Drake, S.A. Drake: A\&A 325, 664 (1997)

R.A. Osten, T.R. Ayres, A. Brown, J.L. Linsky, A. Krishnamurthi: ApJ 582, 1073 (2003)

R.A. Osten, A. Brown: ApJ 515, 746 (1999)

R.A. Osten, A. Brown, T.R. Ayres, S.A. Drake, E. Franciosini, R. Pallavicini, G. Tagliaferri, R.T. Stewart, S.L. Skinner, J.L. Linsky: ApJS 153, 317 (2004) 
R.A. Osten, A. Brown, T.R. Ayres, J.L. Linsky, S.A. Drake, M. Gagné, R.A. Stern: ApJ 544, 953 (2000)

R.A. Osten, A. Brown, B.E. Wood, P. Brady: ApJS 138, 99 (2002)

R. Ottmann: A\&A 273, 546 (1993)

R. Ottmann: A\&A 286, L27 (1994)

R. Ottmann, T.A. Fleming, L. Pasquini: $A \& A$ 322, 785 (1997)

R. Ottmann, M.J. Pfeiffer, T. Gehren: $A \& A$ 338, 661 (1998)

R. Ottmann, J.H.M.M. Schmitt: $A \& A$ 283, 871 (1994)

R. Ottmann, J.H.M.M. Schmitt: $A \& A$ 307, 813 (1996)

R. Ottmann, J.H.M.M. Schmitt, M. Kürster: ApJ 413, 710 (1993)

H. Ozawa, F. Nagase, Y. Ueda, T. Dotani, M. Ishida: ApJ 523, L81 (1999)

R. Pallavicini: A\&AR 1, 177 (1989)

R. Pallavicini: in 11th Cambridge Workshop on Cool Stars, Stellar Systems, and the Sun, eds. R.J. Garcia Lopez, et al., ASP, San Francisco, p. 377 (2001)

R. Pallavicini, L. Golub, R. Rosner, G.S. Vaiana, T. Ayres, J.L. Linsky: ApJ 248, 279 (1981)

R. Pallavicini, B.C. Monsignori-Fossi, M. Landini, J.H.M.M. Schmitt: A\&A 191, 109 (1988)

R. Pallavicini, G. Peres, S. Serio, G. Vaiana, L. Acton, J. Leibacher, R. Rosner: ApJ 270, 270 (1983)

R. Pallavicini, S. Serio, G.S. Vaiana: ApJ 216, 108 (1977)

R. Pallavicini, G. Tagliaferri, A.M.T. Pollock, J.H.M.M. Schmitt, C. Rosso: A\&A 227, 483 (1990b)

R. Pallavicini, G. Tagliaferri, L. Stella: A\&A 228, 403 (1990a)

H.C. Pan, C. Jordan: MNRAS 272, 11 (1995)

H.C. Pan, C. Jordan, K. Makishima, R.A. Stern, K. Hayashida, M. Inda-Koide: MNRAS 285, 735 (1997)

M.R. Panzera, G. Tagliaferri, L. Pasinetti, E. Antonello: A\&A 348, 161 (1999)

E.N. Parker: ApJ 330, 474 (1988)

C.E. Parnell, P.E. Jupp: ApJ 529, 554 (2000)

L. Pasquini, J.H.M.M. Schmitt, R. Pallavicini: $A \& A$ 226, 225 (1989)

B.M. Patten, T. Simon: ApJ 415, L123 (1993)

B.M. Patten, T. Simon: ApJS 106, 489 (1996)

G. Pearce, R.A. Harrison: $A \& A$, 206, 121 (1988)

G. Pearce, R.A. Harrison, B.J.I. Bromage, A.G.M. Pickering: A\&A 253, 601 (1992)

G. Peres, S. Orlando, F. Reale, R. Rosner: ApJ 563, 1045 (2001)

G. Peres, S. Orlando, F. Reale, R. Rosner, H. Hudson: ApJ 528, 537 (2000)

G. Peres, F. Reale, S. Serio, R. Pallavicini: ApJ 312, 895 (1987)

G. Peres, R. Rosner, S. Serio, G.S. Vaiana: ApJ 252, 791 (1982)

M.A.C. Perryman, and the Hipparcos Science Team: The Hipparcos and Tycho Catalogues, ESA report SP-1200, ESA, Noordwijk (1997)

A.A. Pevtsov, G.H. Fisher, L.W. Acton, D.W. Longcope, C.M. Johns-Krull, C.C. Kankelborg, T.R. Metcalf: ApJ 598, 1387 (2003)

K.J.H. Phillips, A.K. Bhatia, H.E. Mason, D.M. Zarro: ApJ 466, 549 (1996)

K.J.H. Phillips, M. Mathioudakis, D.P. Huenemoerder, D.R. Williams, M.E. Phillips, F.P. Keenan: MNRAS 325, 1500 (2001)

I. Pillitteri, G. Micela, S. Sciortino, F. Favata: $A \& A$ 399, 919 (2003)

N. Pizzolato, A. Maggio, G. Micela, S. Sciortino, P. Ventura: A\&A 397, 147 (2003)

N. Pizzolato, A. Maggio, S. Sciortino: $A \& A$ 361, 614 (2000)

G.W. Pneuman: Sol. Phys. 78, 229 (1982)

G. Poletto, R. Pallavicini, R.A. Kopp: A\&A 201, 93 (1988)

A.M.T. Pollock, G. Tagliaferri, R. Pallavicini: $A \& A$ 241, 541 (1991)

D. Porquet, R. Mewe, J. Dubau, A.J.J. Raassen, J.S. Kaastra: A\&A 376, 1113 (2001) 
S.H. Pravdo, L. Angelini: ApJ 447, 342 (1995)

T. Preibisch: $A \& A$ 320, 525 (1997a)

T. Preibisch: $A \& A$ 324, 690 (1997b)

T. Preibisch: $A \& A$ 338, L25 (1998)

T. Preibisch: $A \& A$ 401, 543 (2003a)

T. Preibisch: $A \& A$ 410, 951 (2003b)

Th. Preibisch, R. Neuhäuser, J.M. Alcalá: $A \& A$ 304, L13 (1995)

T. Preibisch, R. Neuhäuser, T. Stanke: A\&A 338, 923 (1998)

T. Preibisch, H. Zinnecker: $A J$ 122, 866 (2001)

T. Preibisch, H. Zinnecker: AJ 123, 1613 (2002)

T. Preibisch, H. Zinnecker, G.H. Herbig: A\&A 310, 456 (1996)

Th. Preibisch, H. Zinnecker, J.H.M.M. Schmitt: A\&A 279, L33 (1993)

P. Preś, M. Siarkowski, J. Sylwester: MNRAS 275, 43 (1995)

C.F. Prosser, S. Randich, J.R. Stauffer, J.H.M.M. Schmitt, T. Simon: AJ 112, 1570 (1996)

C.F. Prosser, J.R. Stauffer, J.-P. Caillault, S. Balachandran, R.A. Stern, S. Randich: AJ 110, 1229 (1995)

J.P. Pye, S.T. Hodgkin, R.A. Stern, J.R. Stauffer: MNRAS 266, 798 (1994)

J.P. Pye, I.M. McHardy: MNRAS 205, 875 (1983)

A.J.J. Raassen, R. Mewe, M. Audard, M. Güdel: A\&A 411, 509 (2003b)

A.J.J. Raassen, R. Mewe, M. Audard, M. Güdel, E. Behar, J.S. Kaastra, R.L.J. van der Meer, C.R. Foley, J.-U. Ness: A\&A 389, 228 (2002)

A.J.J. Raassen, J.-U. Ness, R. Mewe, R.L.J. van der Meer, V. Burwitz, J.S. Kaastra: A\&A 400, 671 (2003a)

S. Randich: in Stellar Clusters and Associations: Convection, Rotation, and Dynamos, eds. R. Pallavicini, G. Micela, S. Sciortino, ASP, San Francisco, p. 401 (2000)

S. Randich, M.S. Giampapa, R. Pallavicini: A\&A 283, 893 (1994)

S. Randich, R. Gratton, R. Pallavicini: A\&A 273, 194 (1993)

S. Randich, J.H.M.M. Schmitt: A\&A 298, 115 (1995)

S. Randich, J.H.M.M. Schmitt, C. Prosser: $A \& A$ 313, 815 (1996b)

S. Randich, J.H.M.M. Schmitt, C.F. Prosser, J.R. Stauffer: A\&A 300, 134 (1995)

S. Randich, J.H.M.M. Schmitt, C.F. Prosser, J.R. Stauffer: $A \& A$ 305, 785 (1996a)

S. Randich, K.P. Singh, T. Simon, S.A. Drake, J.H.M.M. Schmitt: A\&A 337, 372 (1998)

J. Raymond, J.G. Doyle: ApJ 247, 686 (1981)

F. Reale: in Stellar Coronae in the Chandra and XMM-Newton Era, eds. F. Favata, J.J. Drake, ASP, San Francisco, p. 103 (2002)

F. Reale, R. Betta, G. Peres, S. Serio, J. McTiernan: A\&A 325, 782 (1997)

F. Reale, F. Bocchino, G. Peres: $A \& A$ 383, 952 (2002)

F. Reale, M. Güdel, G. Peres, M. Audard: $A \& A$ 416, 733 (2004)

F. Reale, G. Micela: $A \& A$ 334, 1028 (1998)

F. Reale, G. Peres: A\&A 299, 225 (1995)

F. Reale, G. Peres, S. Serio, R. Rosner, J.H.M.M. Schmitt: ApJ 328, 256 (1988)

F. Reale, S. Serio, G. Peres: A\&A 272, 486 (1993)

S. Redfield, R.R. Ayres, J.L. Linsky, T.B. Ake, A.K. Dupree, R.D. Robinson, P.R. Young: ApJ 585, 993 (2003)

N. Reid, S.L. Hawley, M. Mateo: MNRAS 272, 828 (1995)

D. Reimers, M. Hünsch, J.H.M.M. Schmitt, F. Toussaint: A\&A 310, 813 (1996)

D. Reimers, J.H.M.M. Schmitt: ApJ 392, L55 (1992)

T.N. Rengarajan, R.P. Verma: MNRAS 203, 1035 (1983)

R.D. Robinson, V.S. Airapetian, S.P. Maran, K.G. Carpenter: ApJ 469, 872 (1996) 
M. Rodonò, I. Pagano, G. Leto, F. Walter, S. Catalano, G. Cutispoto, G. Umana: A\&A 346, 811 (1999)

R. Rosner, C.-H. An, Z.E. Musielak, R.L. Moore, S.T. Suess: ApJ 372, L91 (1991)

R. Rosner, L., Golub, G.S. Vaiana: ARA\&A 23, 413 (1985)

R. Rosner, Z.E. Musielak, F. Cattaneo, R.L. Moore, S.T. Suess: ApJ 442, L25 (1995)

R. Rosner, W.H. Tucker, G.S. Vaiana: ApJ 220, 643 (1978) = RTV

S.M. Rucinski, R. Mewe, J.S. Kaastra, O. Vilhu, S.M. White: ApJ 449, 900 (1995)

R.E. Rutledge, G. Basri, E.L. Martín, L. Bildstein: ApJ 538, L141 (2000)

R.G.M. Rutten, C.J. Schrijver: A\&A 177, 155 (1987)

R.G.M. Rutten, C.J. Schrijver, A.F.P. Lemmens, C. Zwaan: $A \& A$ 252, 203 (1991)

J.L.R. Saba, J.T. Schmelz, A.K. Bhatia, K.T. Strong: ApJ 510, 1064 (1999)

J. Sanz-Forcada, N.S. Brickhouse, A.K. Dupree: ApJ 554, 1079 (2001)

J. Sanz-Forcada, N.S. Brickhouse, A.K. Dupree: ApJ 570, 799 (2002)

J. Sanz-Forcada, F. Favata, G. Micela: $A \& A$ 416, 281 (2004)

J. Sanz-Forcada, A. Maggio, G. Micela: A\&A 408, 1087 (2003)

I.S. Savanov, I. Tuominen: in The Sun and Cool Stars: Activity, Magnetism, Dynamos, eds. I.

Tuominen, D. Moss, G. Rüdiger, Springer, Berlin, p. 449 (1991)

L. Scelsi, A. Maggio, G. Peres, P. Gondoin: A\&A 413, 643 (2004)

J.T. Schmelz: ApJ 408, 373 (1993)

J.T. Schmelz, J.L.R. Saba, J.C. Chauvin, K.T. Strong: ApJ 477, 509 (1997)

J.H.M.M. Schmitt: ApJS 90, 735 (1994)

J.H.M.M. Schmitt: in Stellar Surface Structure, IAU Sym. 176, eds. K.G. Strassmeier, J.L. Linsky, Kluwer, Dordrecht, p. 85 (1996)

J.H.M.M. Schmitt: $A \& A$ 318, 215 (1997)

J.H.M.M. Schmitt, A. Collura, S. Sciortino, G.S. Vaiana, F.R. Harnden Jr., R. Rosner: ApJ 365, 704 (1990a)

J.H.M.M. Schmitt, J.J. Drake, B.M. Haisch, R.A. Stern: ApJ 467, 841 (1996c)

J.H.M.M. Schmitt, J.J. Drake, R.A. Stern: ApJ 465, L51 (1996a)

J.H.M.M. Schmitt, J.J. Drake, R.A. Stern, B.M. Haisch: ApJ 457, 882 (1996b)

J.H.M.M. Schmitt, F. Favata: Nature 401, 44 (1999)

J.H.M.M. Schmitt, T.A. Fleming, M.S. Giampapa: ApJ 450, 392 (1995)

J.H.M.M. Schmitt, L. Golub, F.R. Harnden Jr., C.W. Maxson, R. Rosner, G.S. Vaiana: ApJ 290, 307 (1985a)

J.H.M.M. Schmitt, M. Güdel, P. Predehl: A\&A 287, 843 (1994b)

J.H.M.M. Schmitt, B.M. Haisch, J.J. Drake: Science 265, 1420 (1994a)

J.H.M.M. Schmitt, F.R. Harnden Jr., G. Peres, R. Rosner, S. Serio: ApJ 288, 751 (1985b)

J.H.M.M. Schmitt, P. Kahabka, J. Stauffer, A.J.M. Pieters: A\&A 277, 114 (1993a)

J.H.M.M. Schmitt, M. Kürster: Science 262, 215 (1993)

J.H.M.M. Schmitt, C. Liefke: A\&A 382, L9 (2002)

J.H.M.M. Schmitt, G. Micela, S. Sciortino, G.S. Vaiana, F.R. Harnden Jr., R. Rosner: ApJ 351, $492(1990 b)$

J.H.M.M. Schmitt, J.-U. Ness: A\&A 388, L13 (2002)

J.H.M.M. Schmitt, J.-U. Ness: A\&A 415, 1099 (2004)

J.H.M.M. Schmitt, J.-U. Ness, G. Franco: A\&A 412, 849 (2003)

J.H.M.M. Schmitt, R. Pallavicini, B.C. Monsignori-Fossi, F.R. Harnden Jr.: A\&A 179, 193 (1987)

J.H.M.M. Schmitt, C. Rosso: A\&A 191, 99 (1988)

J.H.M.M. Schmitt, R.A. Stern, J.J. Drake, M. Kürster: ApJ 464, 898 (1996d)

J.H.M.M. Schmitt, R. Wichmann: Nature 412, 508 (2001)

H.W. Schnopper, J.P. Delvaille, A. Epstein, H. Helmken, S.S. Murray, G. Clark, G. Jernigan, R. Doxsey: ApJ 210, L75 (1976) 
C.J. Schrijver: $A \& A$ 127, 289 (1983)

C.J. Schrijver: $A \& A$ 172, 111 (1987)

C.J. Schrijver, M.J. Aschwanden: ApJ 566, 1147 (2002)

C.J. Schrijver: A\&A 269, 446 (1993)

C.J. Schrijver, J. Coté, C. Zwaan, S.H. Saar: ApJ 337, 964 (1989a)

C.J. Schrijver, A.K. Dobson, R.R. Radick: A\&A 258, 432 (1992)

C.J. Schrijver, B. Haisch: ApJ 456, L55 (1996)

C.J. Schrijver, K.L. Harvey: ApJ 343, 481 (1989)

C.J. Schrijver, J.R. Lemen, R. Mewe: ApJ 341, 484 (1989b)

C.J. Schrijver, R. Mewe, G.H.J. van den Oord, J.S. Kaastra: A\&A 302, 438 (1995)

C.J. Schrijver, R. Mewe, F.M. Walter: $A \& A$ 138, 258 (1984)

C.J. Schrijver, G.H.J. van den Oord, R. Mewe: $A \& A$ 289, L23 (1994)

K.-P. Schröder, M. Hünsch, J.H.M.M. Schmitt: A\&A 335, 591 (1998)

S. Sciortino, F. Damiani, F. Favata, G. Micela: A\&A 332, 825 (1998)

S. Sciortino, F. Favata, G. Micela: $A \& A$ 296, 370 (1995)

S. Sciortino, A. Maggio, F. Favata, S. Orlando: A\&A 342, 502 (1999)

S. Sciortino, G. Micela, F. Damiani, E. Flaccomio, K. Briggs, M. Denby, J. Pye, N. Grosso, A.M.

Read, P. Gondoin, R.D. Jeffries: A\&A (2001) 365, L259

S. Sciortino, G. Micela, F. Favata, A. Spagna, M.G. Lattanzi: A\&A 357, 460 (2000)

S. Serio, G. Peres, G.S. Vaiana, L. Golub, R. Rosner: ApJ 243, 288 (1981)

S. Serio, F. Reale, J. Jakimiec, B. Sylwester, J. Sylwester: A\&A 241, 197 (1991)

J.S. Shaw, J.-P. Caillault, J.H.M.M. Schmitt: ApJ 461, 951 (1996)

A. Shemi: MNRAS 251, 221 (1991)

K. Shibata, T. Yokoyama: ApJ 526, L49 (1999)

K. Shibata, T. Yokoyama: ApJ 577, 422 (2002)

T. Shimizu: PASJ 47, 251 (1995)

M. Siarkowski: MNRAS 259, 453 (1992)

M. Siarkowski, P. Preś, S.A. Drake, N.E. White, K.P. Singh: ApJ 473, 470 (1996)

T. Simon: PASP 112, 599 (2000)

T. Simon, W. Cash, T.P., Snow Jr.: ApJ 293, 542 (1985)

T. Simon, S.A. Drake: ApJ 346, 303 (1989)

T. Simon, S.A. Drake: $A J$ 106, 1660 (1993)

T. Simon, S.A. Drake, P.D. Kim: PASP 107, 1034 (1995)

T. Simon, W. Landsman: ApJ 380, 200 (1991)

T. Simon, W. Landsman: ApJ 483, 435 (1997)

T. Simon, B.M. Patten: PASP 110, 283 (1998)

K.P. Singh, S.A. Drake, E.V. Gotthelf, N.E. White: ApJ 512, 874 (1999)

K.P. Singh, S.A. Drake, N.E. White: ApJ 445, 840 (1995)

K.P. Singh, S.A. Drake, N.E. White: AJ 111, 2415 (1996b)

K.P. Singh, S.A. Drake, N.E. White, T. Simon: AJ 112, 221 (1996c)

K.-P. Singh, S. Slijkhuis, N.J. Westergaard, H.W. Schnopper, Ø. Elgarøy, O. Engvold, P. Joras: MNRAS 224, 481 (1987)

K.P. Singh, N.E. White, S.A. Drake: ApJ 456, 766 (1996a)

S.L. Skinner, M. Gagné, E. Belzer: ApJ 598, 375 (2003)

S.L. Skinner, M. Güdel, M. Audard, K. Smith: ApJ in press (2004)

S.L. Skinner, M. Güdel, K. Koyama, S. Yamauchi: ApJ 486, 886 (1997)

S.L. Skinner, F.M. Walter: ApJ 509, 761 (1998)

S.L. Skinner, S. Yamauchi: ApJ 471, 987 (1996)

A. Skumanich: ApJ 171, 565 (1972)

A. Skumanich: Aust. J. Phys. 38, 971 (1985) 
D.R. Soderblom, J.R. Stauffer, K.B. MacGregor, B.F. Jones: ApJ 409, 624 (1993)

L. Spitzer: Physics of Fully Ionized Gases, Interscience, New York (1962)

K.G. Stassun, D.R. Ardila, M. Barsony, G. Basri, R.D. Mathieu: AJ 127, 3537 (2004)

J.R. Stauffer, J.-P. Caillault, M. Gagné, C.F. Prosser, L.W. Hartmann: ApJS 91, 625 (1994)

B. Stelzer, V. Burwitz: A\&A 402, 719 (2003)

B. Stelzer, V. Burwitz, M. Audard, M. Güdel, J.-U. Ness, N. Grosso, R. Neuhäuser, J.H.M.M. Schmitt, P. Predehl, B. Aschenbach: A\&A 392, 585 (2002)

B. Stelzer, R. Neuhäuser: $A \& A$ 361, 581 (2000)

B. Stelzer, R. Neuhäuser: $A \& A$ 377, 538 (2001)

B. Stelzer, R. Neuhäuser, S. Casanova, T. Montmerle: $A \& A$ 344, 154 (1999)

B. Stelzer, R. Neuhäuser, V. Hambaryan: $A \& A$ 356, 949 (2000)

B. Stelzer, J.H.M.M. Schmitt: $A \& A$ 418, 687 (2004)

K. Stȩpieǹ: $A \& A$ 292, 191 (1994)

K. Stȩpieǹ, J.H.M.M. Schmitt, W. Voges: A\&A 370, 157 (2001)

R.A. Stern, S.K. Antiochos, F.R. Harnden Jr.: ApJ 305, 417 (1986)

R.A. Stern, J.R. Lemen, J.H.M.M. Schmitt, J.P. Pye: ApJ 444, L45 (1995a)

R.A. Stern, J.H.M.M. Schmitt, P.T. Kahabka: ApJ 448, 683 (1995b)

R.A. Stern, J.H.M.M. Schmitt, J.P. Pye, S.T. Hodgkin, J.R. Stauffer, T. Simon: ApJ 427, 808 (1994)

R.A. Stern, J.H.M.M. Schmitt, C. Rosso, J.P. Pye, S.T. Hodgkin, J.R. Stauffer: ApJ 399, L159 (1992c)

R.A. Stern, Y. Uchida, S. Tsuneta, F. Nagase: ApJ 400, 321 (1992a)

R.A. Stern, Y. Uchida, F. Walter, O. Vilhu, D. Hannikainen, A. Brown, A. Vealé, B.M. Haisch: ApJ 391, 760 (1992b)

R.A. Stern, J.H. Underwood, S.K. Antiochos: ApJ 264, L55 (1983)

R.A. Stern, M.-C. Zolcinski, S.K. Antiochos, J.H. Underwood: ApJ 249, 647 (1981)

M.F. Sterzik, J.M. Alcalá, R. Neuhäuser, J.H.M.M. Schmitt: A\&A 297, 418 (1995)

K.G. Strassmeier, J. Bartus: $A \& A$ 354, 537 (2000)

K.M. Strom, S.E. Strom: ApJ 424, 237 (1994)

K.M. Strom, S.E. Strom, F.P. Wilkin, L. Carrasco, I. Cruz-Gonzalez, E. Recillas, A. Serrano, R.L. Seaman, J.R. Stauffer, D. Dai, J. Sottile: ApJ 362, 168 (1990)

P.A. Sturrock, W.W. Dixon, J.A. Klimchuk, S.K. Antiochos: ApJ 356, L31 (1990)

J.H. Swank, N.E. White, S.S. Holt, R.H. Becker: ApJ 246, 208 (1981)

J. Sylwester, J.R. Lemen, R. Mewe: Nature 310, 665 (1984)

B. Sylwester, J. Sylwester, S. Serio, F. Reale, R.D. Bentley, A. Fludra: A\&A 267, 586 (1993)

G. Tagliaferri, S. Covino, G. Cutispoto, R. Pallavicini: A\&A 345, 514 (1999)

G. Tagliaferri, S. Covino, T.A. Fleming, M. Gagné, R. Pallavicini, F. Haardt, Y. Uchida: $A \& A 321$, 850 (1997)

G. Tagliaferri, J.G. Doyle, P. Giommi: A\&A 231, 131 (1990)

G. Tagliaferri, N.E. White, J.G. Doyle, J.L. Culhane, B.J.M. Hassall, J.H. Swank: A\&A 251, 161 (1991)

A. Telleschi, M. Güdel, K. Briggs, M. Audard, J.-U. Ness, S.L. Skinner: ApJ submitted (2004)

P. Testa, J.J. Drake, G. Peres: ApJ in press (2004)

K. Topka, Y. Avni, L. Golub, P. Gorenstein, F.R. Harnden Jr., R. Rosner, G.S. Vaiana: ApJ 259, 677 (1982)

K. Topka, D. Fabricant, F.R. Harnden Jr., P. Gorenstein, R. Rosner: ApJ 229, 661 (1979)

Y. Tsuboi, K. Imanishi, K. Koyama, N. Grosso, T. Montmerle: ApJ 532, 1089 (2000)

Y. Tsuboi, K. Koyama, K. Hamaguchi, K. Tatematsu, Y. Sekimoto, J. Bally, B. Reipurth: ApJ 554, 734 (2001)

Y. Tsuboi, K. Koyama, H. Murakami, M. Hayashi, S. Skinner, S. Ueno: ApJ 503, 894 (1998) 
Y. Tsuboi, Y. Maeda, E.D. Feigelson, G.P. Garmire, G. Chartas, K. Mori, S.H. Pravdo: ApJ 587, L51 (2003)

M. Tsujimoto, K. Koyama, Y. Tsuboi, M. Goto, N. Kobayashi: ApJ 566, 974 (2002)

T. Tsuru, K. Makishima, T. Ohashi, H. Inoue, K. Koyama, M.J.L. Turner, M.A. Marstow, I.M. McHardy, J.P. Pye, H. Tsunemi, S. Kitamoto, A.R. Taylor, R.F. Nelson: PASJ 41, 679 (1989)

T. Tsuru, K. Makishima, T. Ohashi, T. Sakao, J.P. Pye, O.R. Williams, M.A. Barstow, S. Takano: MNRAS 255, 192 (1992)

Y. Uchida, T. Sakurai: in Activity in Red Dwarf Stars, eds. P.B. Byrne, M. Rodonò, Reidel, Dordrecht, p. 629 (1984)

Y. Uchida, T. Sakurai: in Unstable Current Systems and Plasma Instabilities in Astrophysics, eds.

M.R. Kundu, G. Holman, Reidel, Dordrecht, p. 287 (1985)

Y.C. Unruh, M. Jardine: $A \& A$ 321, 177 (1997)

G.S Vaiana: in Solar and Stellar Magnetic Fields: Origins and Coronal Effects, ed. J.O. Stenflo, Reidel, Dordrecht, p. 165 (1983)

G.S. Vaiana, J.P. Cassinelli, G. Fabbiano, R. Giacconi, L. Golub, P. Gorenstein, B.M. Haisch, F.R. Harnden Jr., H.M. Johnson, J.L. Linsky, C.W. Maxson, R. Mewe, R. Rosner, F. Seward, K. Topka, C. Zwaan: ApJ 245, 163 (1981)

G.S. Vaiana, R. Rosner: ARA\&A 15, 363 (1978)

E.J.M. van den Besselaar, A.J.J. Raassen, R. Mewe, R.L.J. van der Meer, M. Güdel, M. Audard: A\&A 411, 587 (2003)

G.H.J. van den Oord, J.G. Doyle, M. Rodonò, D.E. Gary, G.W. Henry, P.B. Byrne, J.L. Linsky, B.M. Haisch, I. Pagano, G. Leto: $A \& A$ 310, 908 (1996)

G.H.J. van den Oord, R. Mewe: A\&A 213, 245 (1989)

G.H.J. van den Oord, R. Mewe, A.C. Brinkman: A\&A 205, 181 (1988)

G.H.J. van den Oord, C.J. Schrijver, M. Camphens, R. Mewe, J.S. Kaastra: A\&A 326, 1090 (1997)

P.W. Vedder, C.R. Canizares: ApJ 270, 666 (1983)

R. Ventura, A. Maggio, G. Peres: A\&A 334, 188 (1998)

J.F. Vesecky, S.K. Antiochos, J.H. Underwood: ApJ 233, 987 (1979) = VAU

O. Vilhu: $A \& A$ 133, 117 (1984)

O. Vilhu, J.-P. Caillault, J. Heise: ApJ 330, 922 (1988)

O. Vilhu, A. Collier Cameron, E. Budding, T. Banks, B. Slee, P. Ehrenfreund, B.H. Foing: $A \& A$ 278, 467 (1993)

O. Vilhu, B. Gustafsson, B. Edvardsson: ApJ 320, 850 (1987)

O. Vilhu, J. Heise: ApJ 311, 937 (1986)

O. Vilhu, S.M. Rucinski: $A \& A$ 127, 5 (1983)

O. Vilhu, F.M. Walter: $A p J$ 321, 958 (1987)

F.M. Walter: ApJ 245, 677 (1981)

F.M. Walter: ApJ 253, 745 (1982)

F.M. Walter: ApJ 274, 794 (1983)

F.M. Walter: ApJ 306, 573 (1986)

F.M. Walter, S. Bowyer: ApJ 245, 671 (1981)

F.M. Walter, A. Brown, R.D. Mathieu, P.C. Myers, F.J. Vrba: AJ 96, 297 (1988)

F.M. Walter, W. Cash, P.A. Charles, C.S. Bowyer: ApJ 236, 212 (1980a)

F.M. Walter, P. Charles, S. Bowyer: Nature 274, 569 (1978a)

F.M. Walter, P. Charles, S. Bowyer: ApJ 225, L119 (1978b)

F.M. Walter, P. Charles, S. Bowyer: AJ 83, 1539 (1978c)

F.M. Walter, D.M. Gibson, G.S. Basri: ApJ 267, 665 (1983)

F.M. Walter, L.V. Kuhi: ApJ 250, 254 (1981)

F.M. Walter, L.V. Kuhi: ApJ 284, 194 (1984)

F.M. Walter, J.L. Linsky, S. Bowyer, G. Garmire: ApJ 236, L137 (1980b) 
F.M. Walter, J.L. Linsky, T. Simon, L. Golub, G.S. Vaiana: ApJ 281, 815 (1984)

F.M. Walter, F.J. Vrba, R.D. Mathieu, A. Brown, P.C. Myers: AJ 107, 692 (1994)

F.M. Walter, F.J. Vrba, S.J. Wolk, R.D. Mathieu, R. Neuhäuser: AJ 114, 1544 (1997)

Y.-M. Wang: ApJ 464, L91 (1996)

N.O. Weiss: in Physics of Solar and Stellar Coronae, eds. J.L. Linsky, S. Serio, Kluwer, Dordrecht, p. 541 (1993)

A.D. Welty, L.W. Ramsey: AJ 108, 299 (1994)

N.E. White: in 9th Cambridge Workshop on Cool Stars, Stellar Systems, and the Sun, eds. R. Pallavicini and A.K. Dupree, ASP, San Francisco, p. 193 (1996)

N.E. White, K. Arnaud, C.R. Day, K. Ebisawa, E.V. Gotthelf, K. Mukai, Y. Soong, T. Yaqoob, A. Antunes: PASJ 46, L97 (1994)

N.E. White, J.L. Culhane, A.N. Parmar, B.J. Kellett, S. Kahn, G.H.J. van den Oord, J. Kuijpers: ApJ 301, 262 (1986)

N.E. White, S.S. Holt, R.H. Becker, E.A. Boldt, P.J. Serlemitsos: ApJ 239, L69 (1980)

N.E. White, F.E. Marshall: ApJ 268, L117 (1983)

N.E. White, P.W. Sanford, E.J. Weiler: Nature 274, 569 (1978)

N.E. White, R.A. Shafer, K. Horne, A.N. Parmar, J.L. Culhane: ApJ 350, 776 (1990)

S.M. White, J. Lim, S.M. Rucinski, C. Roberts, D. Kilkenny, S.G. Ryan, P. Prado, M.R. Kundu: in Astrophysics in the Extreme Ultraviolet, eds. S. Bowyer, R.F. Malina, Kluwer, Dordrecht, p. 165 (1996)

D.R. Whitehouse: A\&A 145, 449 (1985)

R. Wichmann, J. Krautter, J.H.M.M. Schmitt, R. Neuhäuser, J.M. Alcalá, H. Zinnecker, R.M. Wagner, R. Mundt, M.F. Sterzik: A\&A 312, 439 (1996)

R. Wichmann, M. Sterzik, J. Krautter, A. Metanomski, W. Voges: A\&A 326, 211 (1997)

G.L. Withbroe: Solar Phys. 45, 301 (1975)

B.E. Wood, A. Brown, J.L. Linsky, B.J. Kellett, G.E. Bromage, S.T. Hodgkin, J.P. Pye: ApJS 93, 287 (1994)

S. Yamauchi, K. Koyama, M. Sakano, K. Okada: PASJ 48, 719 (1996)

H. Zinnecker, T. Preibisch: A\&A 292, 152 (1994) 